\title{
Construction of Vicinal Quaternary Centers via Iridium- Catalyzed Asymmetric Allenylic Alkylation of Racemic Tertiary Alcohols
}

\author{
Mayuko Isomura, David A. Petrone, and Erick M. Carreira* \\ <erickm.carreira@org.chem.ethz.ch> \\ Eidgenössische Technische Hochschule Zürich, Vladimir-Prelog-Weg 3, \\ HCI, 8093 Zürich, Switzerland.
}

Supporting Information

\section{Contents}

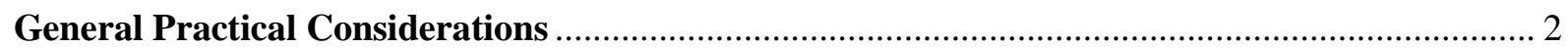

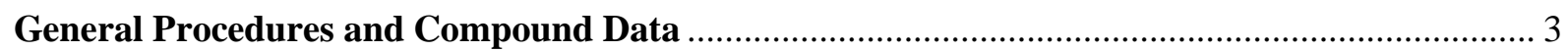

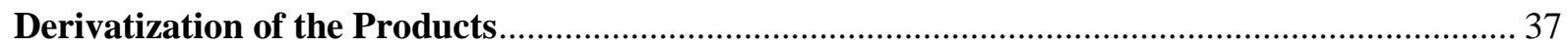

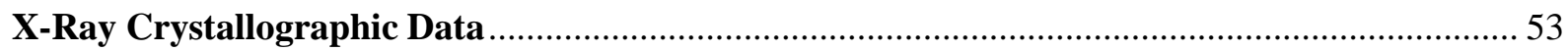

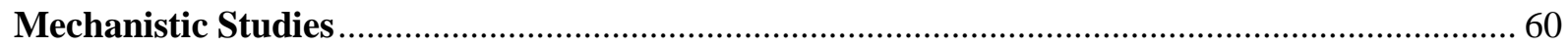

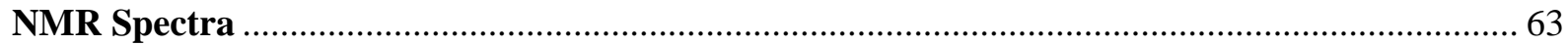

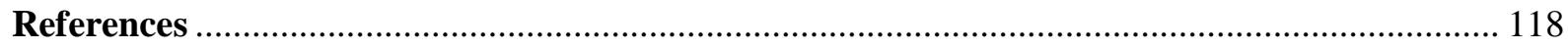




\section{General Practical Considerations}

Unless otherwise stated, all catalytic reactions were carried out under an inert atmosphere of dry nitrogen utilizing glassware that was oven $\left(120^{\circ} \mathrm{C}\right)$ or flame dried, whereas the work-up and isolation of the products from the catalytic reactions were conducted on the bench-top using standard techniques. Reactions were monitored using thin-layer chromatography (TLC) on EMD Silica Gel 60 F254 plates. Visualization of the developed plates was performed under UV light $(254 \mathrm{~nm})$ or using $\mathrm{KMnO}_{4}$. Unless otherwise stated, tetrahydrofuran, toluene, diethyl ether, 1,4-dioxane, ethyl acetate, dichloromethane and chloroform, were obtained in anhydrous form over $4 \AA$ MS from Fischer Scientific, whereas tert-butyl methyl ether, $\alpha, \alpha, \alpha-$ trifluorotoluene, 1,2-dimethoxyethane, $o$-, $m$ - and $p$-xylene, and $n$-hexane were obtained as reagent grade from commercial sources and dried by storing over $4 \AA$ MS (activated for 12 hours at $160{ }^{\circ} \mathrm{C}$ at $0.5 \mathrm{mmHg}$ ) for 24 hours. Reagent grade $N, N$-diisopropyl amine was used as received. Unless otherwise stated, silica gel flash column chromatography ${ }^{1}$ was performed on Silicycle 230-400 mesh silica gel. NMR characterization data was collected at $296 \mathrm{~K}$ on a Bruker Avance III, Bruker Ascend, Bruker DRX, or Bruker NEO operating at $400 \mathrm{MHz}$ for ${ }^{1} \mathrm{H}$ NMR, $100 \mathrm{MHz}$ for ${ }^{13} \mathrm{C}$ NMR, $243 \mathrm{MHz}$ for ${ }^{31} \mathrm{P}$ NMR, $375 \mathrm{MHz}$ for ${ }^{19} \mathrm{~F}$ NMR, and $500 \mathrm{MHz}$ for nOe experiments. ${ }^{1} \mathrm{H}$ NMR spectra were internally referenced to the residual solvent signal $\left(\mathrm{CDCl}_{3}=7.26 \mathrm{ppm}\right)$ or TMS. ${ }^{13} \mathrm{C} \mathrm{NMR}$ spectra were internally referenced to the residual solvent signal $\left(\mathrm{CDCl}_{3}=77.0 \mathrm{ppm}\right)$ and are reported as observed. Data for ${ }^{1} \mathrm{H}$ NMR are reported as follows: chemical shift $(\delta \mathrm{ppm})$, multiplicity $(\mathrm{s}=$ singlet, $\mathrm{d}=$ doublet, $\mathrm{t}=$ triplet, $\mathrm{q}=$ quartet, $\mathrm{m}=$ multiplet, $\mathrm{br}=$ broad $)$, coupling constant $(\mathrm{Hz})$, integration. Melting point ranges were determined on a Büchi B545 Melting Point Apparatus. NMR yields for the optimization section were obtained by ${ }^{1} \mathrm{H}$ NMR analysis of the crude reaction mixture using 5 second delay and 1,1,2,2-tetrachloroethane as an internal standard. Infrared spectra were recorded neat on a Perkin-Elmer Spectrum Two FT-IR spectrometer. The peaks are reported as absorption maxima $\left(\mathrm{n}, \mathrm{cm}^{-1}\right)$. High resolution mass spectra data were obtained at the mass spectrometry service in the Laboratory für Organisch Chemie at ETH Zürich on VG-TRIBRIB for electron impact ionization (EI), a Varian IonSpec Spectrometer for electrospray ionization (ESI) or an IonSpec Ultima Fourier Transform Mass Spectrometer for matrix-assisted laser desorption/ionization (MALDI) and are reported as $(\mathrm{m} / \mathrm{z}$ ). Enantiomeric excesses were determined on a Jasco2080 Plus supercritical fluid chromatography (SFC) or a Waters e2695 separation module (HPLC). Optical rotations were measured with Jasco P-2000 Polarimeter, $10 \mathrm{~cm}, 1.5 \mathrm{~mL}$ cell. Single crystals for X-ray diffraction were measured on XtaLAB Synergy, Dualflex, Pilatus 300K diffractometer with CuK $\alpha$ radiation $(\lambda=1.54184 \AA$ ). All measurements were carried out at 100 K. The structures were solved using SHELXS, ${ }^{2}$ SHELXT $^{3}$ or Superflip ${ }^{4}$ and refined by fullmatrix least-squares analysis (SHELXL) using the program package OLEX2. ${ }^{5}$ Unless otherwise indicated below, all non-hydrogen atoms were refined anisotropically and hydrogen atoms were constrained to ideal geometries and refined with fixed isotropic displacement parameters (in terms of a riding model). The crystallographic data can be obtained free of charge S3 from The Cambridge Crystallographic Data Centre, 12 Union Road, Cambridge CB2 1EZ, UK or via https://www.ccdc.cam.ac.uk/getstructures. Solid-state structures were visualized using the ORTEP3 program. ${ }^{6}$ 


\section{General Procedures and Compound Data}

\section{General Synthetic Remarks}

All standard reagents were purchased from ABCR, ACROS, Sigma Aldrich, Fluka, TCI, Strem, Alfa, Combi-Blocks or Fluorochem, and were used without further purification unless otherwise mentioned. La(OTf) 3 was dried in vacuo (5 Torr) at $180{ }^{\circ} \mathrm{C}$ for 24 hours, and stored in a desiccator. Tertiary alcohol starting materials 1a-1o, 1r-1t are synthesized according to known procedure. ${ }^{7}$

\section{Preparation of Tertiary Alcohol Starting Material 1p}

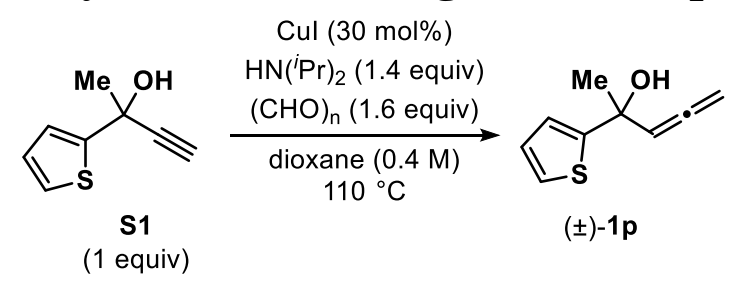

2-(thiophen-3-yl)penta-3,4-dien-2-ol (1p): Prepared via the procedure of Luo and $\mathrm{Ma}^{8}{ }^{8}$ A 100 $\mathrm{mL}$ round-bottom flask containing a magnetic stir-bar was charged with $\mathbf{S 1}^{9}$ (787 $\mathrm{mg}, 5.17$ mmol, 1 equiv), CuI (296 mg, $1.55 \mathrm{mmol}, 30 \mathrm{~mol} \%$ ), paraformaldehyde ( $249 \mathrm{mg}, 8.28 \mathrm{mmol}$, 1.6 equiv), $N, N$-diisopropylamine $(1.03 \mathrm{~mL}, 7.24 \mathrm{mmol}, 1.4$ equiv), which was taken up with reagent grade 1,4-dioxane $(13 \mathrm{~mL})$. The flask was fitted with a water-cooled reflux condenser and the reaction vessel was heated at $110^{\circ} \mathrm{C}$ without the exclusion of air/moisture for 40 hours. The reaction was then cooled, the stir-bar was removed, the reaction mixture was filtered through a plug of silica gel $\left(5 \mathrm{~cm} \times 5 \mathrm{~cm}\right.$, aided by house vacuum) and eluted with $\mathrm{Et}_{2} \mathrm{O}$. After removal of all volatiles by rotary evaporation, the crude residue was taken up in $\mathrm{Et}_{2} \mathrm{O}(20 \mathrm{~mL})$. With the aid of swirling, a significant amount of off-white precipitate was formed and the mixture was again filtered through silica gel and eluted with $\mathrm{Et}_{2} \mathrm{O}$. The title compound was purified via $\mathrm{SiO}_{2}$ flash column chromatography using pentane:Et $2 \mathrm{O}(5: 1 \mathrm{v}: \mathrm{v})$ as the mobile phase, and was obtained as a clear oil (711 mg, $4.27 \mathrm{mmol}, 83 \%$ yield). Spectral data was in accordance with the reported literature. ${ }^{10} \mathbf{H} \mathbf{~ N M R}\left(400 \mathrm{MHz}, \mathrm{CDCl}_{3}\right) \delta 7.22(\mathrm{dd}, J=5.0,1.3$ $\mathrm{Hz}, 1 \mathrm{H}), 7.02-6.91(\mathrm{~m}, 2 \mathrm{H}), 5.64(\mathrm{t}, J=6.6 \mathrm{~Hz}, 1 \mathrm{H}), 5.02-4.98(\mathrm{~m}, 2 \mathrm{H}), 2.36(\mathrm{~s}, 1 \mathrm{H}), 1.76$ $(\mathrm{s}, 3 \mathrm{H}) .{ }^{13} \mathrm{C}$ NMR $\left(100 \mathrm{MHz}, \mathrm{CDCl}_{3}\right) \delta 205.4,152.3,126.6,124.4,122.9,100.1,79.8,71.4$, 30.8 .

\section{Preparation of Tertiary Alcohol Starting Material 1q}

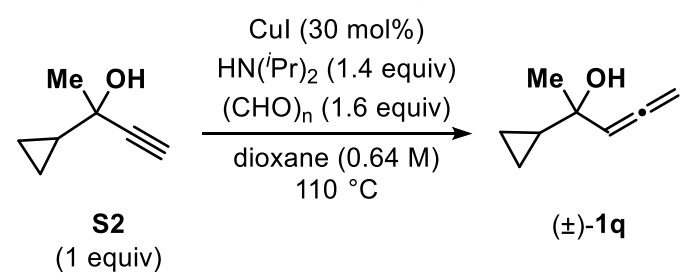

2-cyclopropylpenta-3,4-dien-2-ol (1q): Prepared via the procedure of Luo and Ma. ${ }^{8}$ A $250 \mathrm{~mL}$ round-bottom flask containing a magnetic stir-bar was charged with $\mathbf{S 2}^{11}$ (1.76 g, $16.0 \mathrm{mmol}, 1$ equiv), $\mathrm{CuI}$ (0.91 g, $4.79 \mathrm{mmol}, 30 \mathrm{~mol} \%)$, paraformaldehyde ( $0.77 \mathrm{~g}, 25.6 \mathrm{mmol}, 1.6$ equiv), $N, N$-diisopropylamine ( $3.19 \mathrm{~mL}, 22.4 \mathrm{mmol}, 1.4$ equiv), which was taken up with reagent grade 1,4-dioxane $(25 \mathrm{~mL})$. The flask was fitted with a water-cooled reflux condenser and there reaction vessel was heated at $110{ }^{\circ} \mathrm{C}$ without the exclusion of air/moisture for 28 hours. The reaction was then cooled, the stir-bar was removed, the reaction mixture was filtered through a plug of silica gel $\left(5 \mathrm{~cm} \times 5 \mathrm{~cm}\right.$, aided by house vacuum) and eluted with $\mathrm{Et}_{2} \mathrm{O}$. After removal 
of all volatiles by rotary evaporation, the crude residue was taken up in $\mathrm{Et}_{2} \mathrm{O}(50 \mathrm{~mL})$. With the aid of swirling, a significant amount of off-white precipitate was formed and the mixture was again filtered through silica gel and eluted with $\mathrm{Et}_{2} \mathrm{O}$. The title compound was purified via $\mathrm{SiO}_{2}$ flash column chromatography using pentane: $\operatorname{Et}_{2} \mathrm{O}(5: 1 \mathrm{v}: \mathrm{v})$ as the mobile phase, and was obtained as a clear oil $(1.11 \mathrm{~g}, 8.94 \mathrm{mmol}, 56 \%$ yield $) .{ }^{1} \mathbf{H} \mathbf{~ N M R}\left(400 \mathrm{MHz}, \mathrm{CDCl}_{3}\right) \delta 5.21(\mathrm{t}$, $J=6.7 \mathrm{~Hz}, 1 \mathrm{H}), 4.93-4.84(\mathrm{~m}, 2 \mathrm{H}), 1.54(\mathrm{br}, 1 \mathrm{H}), 1.33(\mathrm{~s}, 3 \mathrm{H}), 1.12-1.02(\mathrm{~m}, 1 \mathrm{H}), 0.48-$ $0.26(\mathrm{~m}, 4 \mathrm{H}) .{ }^{13} \mathbf{C}$ NMR $\left(100 \mathrm{MHz}, \mathrm{CDCl}_{3}\right) \delta 205.9,98.0,78.3,70.4,27.6,21.9,1.0,0.8$. IR (atr, cm-1) 3400, 3084, 3008, 2977, 2930, 1956, 1451, 1371, 1304, 1155, 1104, 1042, 1019, 921, 840, 750, 729, 622, 553, 500. HRMS (EI+, M-H) Calc'd for $\mathrm{C}_{8} \mathrm{H}_{11} \mathrm{O}$ 123.0804, found 123.0805 . 
General procedure 1: Preparation of silyl ketene acetals

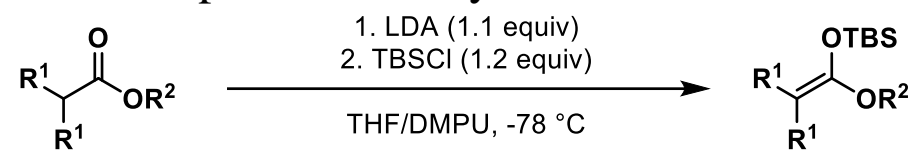

General Procedure 1a - To a solution of diisopropylamine (1.2 equiv) in THF ( $0.66 \mathrm{M})$ cooled to $0{ }^{\circ} \mathrm{C}$ was added ${ }^{n} \mathrm{BuLi}$ (1.6 $\mathrm{M}$ in hexane, 1.1 equiv) dropwise over period of 5 minutes. The solution was stirred with this temperature for 20 minutes, cooled to $-78^{\circ} \mathrm{C}$, and ester ( 1 equiv) was dropwise added. After stirring the solution for 1 hour, DMPU (5 M in THF) was added followed by the dropwise addition of TBSCl (1.2 equiv, $3 \mathrm{M}$ in THF). The resulting solution was stirred at this temperature for 30 minutes, and then the reaction was allowed to warm to room temperature where it was stirred for an additional 12 hours. At this time, the reaction was quenched with water, and the organic solvents were concentrated in vacuo. The organics were extracted with pentane, and combined organic layers were washed with water, saturated aqueous $\mathrm{NaHCO}_{3}$, and brine. The resulting solution was dried over $\mathrm{Na}_{2} \mathrm{SO}_{4}$, filtered, and concentrated in vacuo. The mixture was purified via vacuum distillation at the indicated pressure and temperature. Note: the reactivity of TBS-ketene acetals was found to differ depends on the batch of TBSCl. High reproducibility was observed with neutralized ketene acetals by passing thorough a plug of basic alumina.

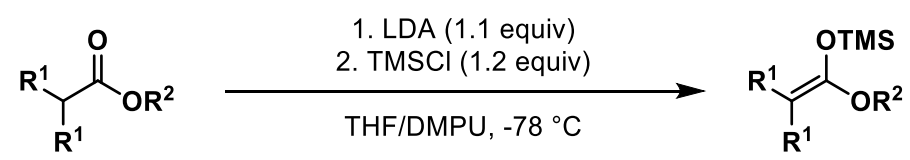

General Procedure 1b - To a solution of diisopropylamine (1.2 equiv) in THF ( $0.66 \mathrm{M})$ cooled to $0{ }^{\circ} \mathrm{C}$, was added ${ }^{n} \mathrm{BuLi}$ (1.6 $\mathrm{M}$ in hexane, 1.1 equiv) dropwise over period of 5 minutes. The solution was stirred with this temperature for 20 minutes, cooled to $-78{ }^{\circ} \mathrm{C}$, and the ester $(1$ equiv) was dropwise added. After stirring the solution for 1 hour, DMPU (5 M in THF) was added followed by TMSCl (1.2 equiv) in a dropwise fashion. The resulting solution was at this temperature for 30 minutes, and then the reaction was allowed to warm to room temperature where it was stirred for an additional 12 hours. At this time, the reaction was quenched with water, and the organic solvents were removed in vacuo. The aqueous phase was extracted with pentane, and the combined organic layers were washed with water, saturated aqueous $\mathrm{NaHCO}_{3}$, and brine. The resulting solution was dried over $\mathrm{Na}_{2} \mathrm{SO}_{4}$, filtered, and concentrated in vacuo. The mixture was purified via vacuum distillation at the indicated pressure and temperature.

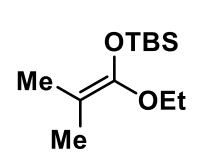

tert-butyl((1-ethoxy-2-methylprop-1-en-1-yl)oxy)dimethylsilane (2a) - Was synthesized according to general procedure 1a using ethyl isobutyrate $(6.68 \mathrm{~mL}$, $50 \mathrm{mmol}, 1$ equiv). The title compound was isolated via vacuum distillation (b.p. $52{ }^{\circ} \mathrm{C}, 5$ Torr $)$ as a clear colorless liquid $(8.70 \mathrm{~g}, 37.6 \mathrm{mmol}, 75 \%$ yield $){ }^{\mathbf{1}} \mathbf{H}$ NMR $\left(400 \mathrm{MHz}, \mathrm{CDCl}_{3}\right) \delta 3.77(\mathrm{q}, J=7.1 \mathrm{~Hz}, 2 \mathrm{H}), 1.58(\mathrm{~s}, 3 \mathrm{H}), 1.54(\mathrm{~s}, 3 \mathrm{H}), 1.22(\mathrm{t}, J=7.1 \mathrm{~Hz}$, 3H), 0.95 (s, 9H), 0.14 (s, 6H). ${ }^{13}$ C NMR (100 MHz, $\left.\mathrm{CDCl}_{3}\right) \delta 148.4,92.1,64.9,25.7$ (3C), 18.0, 16.9, 16.5, 14.9, -4.6 (2C). IR (atr, $\left.\mathrm{cm}^{-1}\right)$ 2958, 2930, 2859, 1702, 1472, 1390, 1362, 1252, 1189, 1178, 1160, 1108, 1042, 981, 853, 837, 780, 682, 542. HRMS (EI+, M+) Calc'd for $\mathrm{C}_{12} \mathrm{H}_{26} \mathrm{O}_{2} \mathrm{Si} 230.1697$, found 230.1697 . 


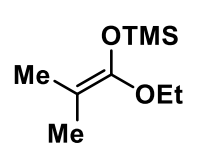

((1-ethoxy-2-methylprop-1-en-1-yl)oxy)trimethylsilane $\quad(2 b) \quad-\quad$ Was synthesized according to general procedure $1 \mathrm{~b}$ using ethyl isobutyrate $(6.68 \mathrm{~mL}$, $50 \mathrm{mmol}, 1 \mathrm{~b}$ equiv). The title compound was isolated via Kugelrohr distillation $\left(75{ }^{\circ} \mathrm{C}, 17 \mathrm{mmHg}\right)$ as a clear colorless liquid $(7.79 \mathrm{~g}, 41.4 \mathrm{mmol}, 83 \%$ yield $)$ Spectral data was in accordance with the reported literature. ${ }^{12}{ }^{1} \mathbf{H} \mathbf{~ N M R}\left(400 \mathrm{MHz}, \mathrm{CDCl}_{3}\right) \delta$ $3.76(\mathrm{q}, J=7.1 \mathrm{~Hz}, 2 \mathrm{H}), 1.57(\mathrm{~s}, 3 \mathrm{H}), 1.52(\mathrm{~s}, 3 \mathrm{H}), 1.21(\mathrm{t}, J=7.1 \mathrm{~Hz}, 3 \mathrm{H}), 0.19(\mathrm{~s}, 9 \mathrm{H}) .{ }^{13} \mathrm{C}$ NMR $\left(100 \mathrm{MHz}, \mathrm{CDCl}_{3}\right) \delta 147.9,91.8,64.3,16.9,16.4,14.8,0.0(3 \mathrm{C})$.

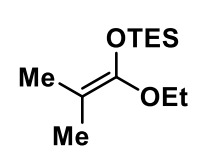

((1-ethoxy-2-methylprop-1-en-1-yl)oxy)triethylsilane (2c) - Was synthesized according to general procedure $1 \mathrm{~b}$ using ethyl isobutyrate $(6.68 \mathrm{~mL}, 50 \mathrm{mmol}, 1$ equiv) and TES-Cl (10.1 mL, $60 \mathrm{mmol}, 1.2$ equiv) instead of TMS-Cl. The title compound was isolated via vacuum distillation (b.p. $60{ }^{\circ} \mathrm{C}, 5$ Torr) as a clear colorless liquid $(5.84 \mathrm{~g}, 31.0 \mathrm{mmol}$, 62\% yield) Spectral data was in accordance with the reported literature. ${ }^{13}{ }^{1} \mathbf{H}$ NMR $\left(400 \mathrm{MHz}, \mathrm{CDCl}_{3}\right) \delta 3.79(\mathrm{q}, J=7.1 \mathrm{~Hz}, 2 \mathrm{H}), 1.59(\mathrm{~s}, 3 \mathrm{H}), 1.57$ $(\mathrm{s}, 3 \mathrm{H}), 1.25(\mathrm{t}, J=7.1 \mathrm{~Hz}, 3 \mathrm{H}), 1.01(\mathrm{t}, J=7.9 \mathrm{~Hz}, 9 \mathrm{H}), 0.71(\mathrm{q}, J=7.9,0.8 \mathrm{~Hz}, 6 \mathrm{H}) .{ }^{13} \mathrm{C}$ NMR $\left(100 \mathrm{MHz}, \mathrm{CDCl}_{3}\right) \delta 148.4,91.8,64.9,16.9,16.5,14.9,6.6(3 \mathrm{C}), 5.0(3 \mathrm{C})$.

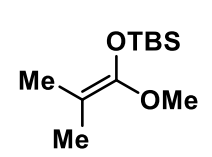

tert-butyl((1-ethoxy-2-methylprop-1-en-1-yl)oxy)dimethylsilane (2d) - Was synthesized according to general procedure 1a using methyl isobutyrate (5.00 $\mathrm{mL}, 43.6 \mathrm{mmol}, 1$ equiv). The title compound was isolated via vacuum distillation (b.p. $46{ }^{\circ} \mathrm{C}, 5$ Torr) as a clear colorless liquid $(6.91 \mathrm{~g}, 31.9 \mathrm{mmol}$, $73 \%$ yield). Spectral data was in accordance with the reported literature. ${ }^{14}{ }^{1} \mathbf{H}$ NMR $(400 \mathrm{MHz}$, $\left.\mathrm{CDCl}_{3}\right) \delta 3.51(\mathrm{~s}, 3 \mathrm{H}), 1.57(\mathrm{~s}, 3 \mathrm{H}), 1.53(\mathrm{~s}, 3 \mathrm{H}), 0.96(\mathrm{~s}, 9 \mathrm{H}), 0.14(\mathrm{~s}, 6 \mathrm{H}),{ }^{13} \mathbf{C}$ NMR (100 $\left.\mathrm{MHz}, \mathrm{CDCl}_{3}\right) \delta 149.7,91.4,57.0,25.7$ (3C), 18.1, 16.9, 16.2, -4.6 (2C).

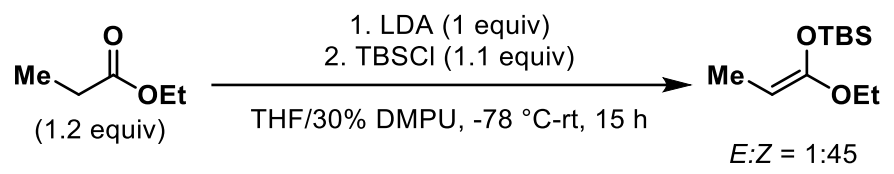

(Z)-tert-butyl((1-ethoxyprop-1-en-1-yl)oxy)dimethylsilane [(Z)-2e] - Prepared via Ireland's procedure: ${ }^{6}$ To a solution of diisopropylamine $(4.75 \mathrm{~mL}, 33.3 \mathrm{mmol}, 1$ equiv) in THF $(24 \mathrm{~mL})$ cooled to $0{ }^{\circ} \mathrm{C}$ was added ${ }^{n} \mathrm{BuLi}(20.8 \mathrm{~mL}, 33.3 \mathrm{mmol}, 1.6 \mathrm{M}$ in hexane, 1.0 equiv) dropwise over period of 5 minutes. The solution was stirred with this temperature for 3 minutes, cooled to $-78^{\circ} \mathrm{C}$, and DMPU ( $\left.30 \mathrm{~mL}\right)$ was added. To that solution was added THF solution $(40 \mathrm{~mL}, 1$ M) of ethyl propionate ( $4.59 \mathrm{~mL}, 1$ equiv) was dropwise added over period of 15 minutes. After stirring the solution for 15 minutes, the solution of TBS-Cl (5.53 g, $36.7 \mathrm{mmol}, 1.1$ equiv) in hexane $(6 \mathrm{~mL})$ was dropwise added to the reaction mixture. The resulting solution was stirred at $-78{ }^{\circ} \mathrm{C}$ for 30 minutes, allowed to warm to room temperature, and stirred for 18 hours. At this time, the reaction was quenched with saturated aqueous $\mathrm{NaHCO}_{3}$ and diluted with pentane. The organic layer was washed with water, dried over $\mathrm{Na}_{2} \mathrm{SO}_{4}$, filtered, and concentrated in vасио. The title compound was isolated via vacuum distillation (b.p. $62{ }^{\circ} \mathrm{C}, 10$ Torr) as a clear colorless liquid (1.82 g, $8.41 \mathrm{mmol}, 25 \%$ yield, $(E: Z=1: 45)$. Spectral data was in accordance with the reported literature. ${ }^{15}{ }^{1} \mathbf{H}$ NMR $\left(400 \mathrm{MHz}, \mathrm{CDCl}_{3}\right) \delta 3.67(\mathrm{q}, J=7.0 \mathrm{~Hz}, 2 \mathrm{H}), 3.43(\mathrm{q}$, $J=6.4 \mathrm{~Hz}, 1 \mathrm{H}), 1.52(\mathrm{~d}, J=6.4 \mathrm{~Hz}, 3 \mathrm{H}), 1.26(\mathrm{t}, J=7.0 \mathrm{~Hz}, 3 \mathrm{H}), 0.94(\mathrm{~s}, 9 \mathrm{H}), 0.16(\mathrm{~s}, 6 \mathrm{H})$. ${ }^{13} \mathrm{C}$ NMR $\left(101 \mathrm{MHz}, \mathrm{CDCl}_{3}\right) \delta 155.8,70.1,62.7,25.7$ (3C), 18.1, 14.5, 9.6, -4.2 (2C). 


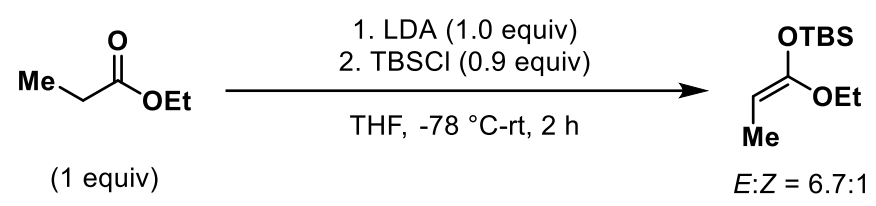

(E)-tert-butyl((1-ethoxyprop-1-en-1-yl)oxy)dimethylsilane $[(E)$-2e $]$ - Prepared via the Ireland's procedure ${ }^{6}$ : To a solution of diisopropylamine $(7.13 \mathrm{~mL}, 50.0 \mathrm{mmol}, 1.0$ equiv) in THF (50 mL) cooled to $0{ }^{\circ} \mathrm{C}$ was added ${ }^{n} \mathrm{BuLi}(31.3 \mathrm{~mL}, 50 \mathrm{mmol}, 1.6 \mathrm{M}$ in hexane, 1.0 equiv) dropwise over period of 5 minutes. The solution was stirred with this temperature for 3 minutes, cooled to $-78^{\circ} \mathrm{C}$, and THF solution $(50 \mathrm{~mL}, 1 \mathrm{M})$ of ethyl propionate $(5.73 \mathrm{~mL}, 1$ equiv) was dropwise added over period of 2 minutes. After stirring the solution for 25 minutes, the solution of TBS-Cl (6.78 g, $45.0 \mathrm{mmol}, 0.9$ equiv) in hexane $(30 \mathrm{~mL})$ was dropwise added to the reaction mixture, which was followed by addition of DMPU $(10 \mathrm{~mL})$. The resulting solution was stirred at $-78{ }^{\circ} \mathrm{C}$ for 10 minutes, allowed to warm to room temperature, where it was stirred for 2 hours. At this time, the organic solvents were removed in vacuo, diluted with pentane, washed with water, saturated aqueous $\mathrm{NaHCO}_{3}$, and brine. The organic layer was dried over $\mathrm{Na}_{2} \mathrm{SO}_{4}$, filtered, and concentrated in vacuo. The title compound was isolated via vacuum distillation (b.p. $50{ }^{\circ} \mathrm{C}, 5$ Torr) as a clear colorless liquid $(2.88 \mathrm{~g}, 13.31 \mathrm{mmol}, 27 \%$ yield) as a mixture of regioisomers $(E: Z=6.7: 1)$. Spectral data was in accordance with the reported literature. ${ }^{6} \mathbf{H}$ NMR $\left(400 \mathrm{MHz}, \mathrm{CDCl}_{3}\right) \delta 3.86(\mathrm{q}, J=7.1 \mathrm{~Hz}, 2 \mathrm{H}), 3.73(\mathrm{q}, J=6.6 \mathrm{~Hz}, 1 \mathrm{H}), 1.50(\mathrm{~d}, J=6.7$ $\mathrm{Hz}, 3 \mathrm{H}), 1.23(\mathrm{t}, J=7.1 \mathrm{~Hz}, 3 \mathrm{H}), 0.93(\mathrm{~s}, 9 \mathrm{H}), 0.17(\mathrm{~s}, 6 \mathrm{H}) \cdot{ }^{13} \mathbf{C ~ N M R}\left(100 \mathrm{MHz}, \mathrm{CDCl}_{3}\right) \delta$ $153.2,80.2,62.9,25.6(3 \mathrm{C}), 18.1,14.9,9.6,-5.0(2 \mathrm{C})$.

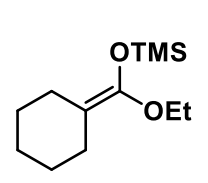

tert-butyl(cyclohexylidene(ethoxy)methoxy)dimethylsilane $(2 \mathrm{~g}) \quad-\quad$ Was synthesized according to general procedure $1 \mathrm{~b}$ using ethyl cyclohexanecarboxylate $(5.00 \mathrm{~g}, 32.0 \mathrm{mmol}, 1$ equiv). The title compound was isolated via vacuum distillation (b.p. $69^{\circ} \mathrm{C}, 15 \mathrm{mbar}$ ) as a clear colorless liquid (2.38 g, $10.4 \mathrm{mmol}, 33 \%$ yield) ${ }^{\mathbf{1}} \mathbf{H}$ NMR $\left(400 \mathrm{MHz} \mathrm{CDCl}_{3}\right) \delta 3.77$ (q, $\left.J=7.1 \mathrm{~Hz}, 2 \mathrm{H}\right), 2.14-$ $2.08(\mathrm{~m}, 2 \mathrm{H}), 2.06-2.00(\mathrm{~m}, 2 \mathrm{H}), 1.52-1.41(\mathrm{~m}, 6 \mathrm{H}), 1.21(\mathrm{t}, J=7.1 \mathrm{~Hz}, 3 \mathrm{H}), 0.20(\mathrm{~s}, 9 \mathrm{H})$. ${ }^{13} \mathbf{C ~ N M R}\left(100 \mathrm{MHz}, \mathrm{CDCl}_{3}\right) \delta 145.6,100.5,64.5,27.7,27.3,27.2,26.8,26.8,14.7,-0.0$ (3C). IR $\left(\operatorname{atr}, \mathrm{cm}^{-1}\right)$ 2960, 2921, 2851, 1696, 1447, 1249, 1181, 1153, 1181, 1059, 1020, 965, 902, 866, 841, 753, 693, 488, 515. HRMS (EI+, M+) Calc'd for $\mathrm{C}_{12} \mathrm{H}_{24} \mathrm{O}_{2} \mathrm{Si}$ 228.1540, found 228.1540 .

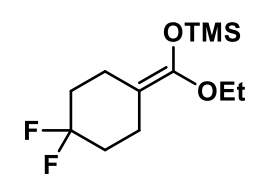

((4,4-difluorocyclohexylidene)(ethoxy)methoxy)trimethylsilane (2h) - To a solution of diisopropylamine ( $3.54 \mathrm{~mL}, 24.8 \mathrm{mmol}, 1.2$ equiv) in THF (40 mL) cooled to $0{ }^{\circ} \mathrm{C}$ was added ${ }^{n} \mathrm{BuLi}(14.3 \mathrm{~mL}, 22.9 \mathrm{mmol}, 1.6 \mathrm{M}$ in hexane, 1.1 equiv) dropwise over period of 5 minutes. The solution was stirred with this temperature for 20 minutes, cooled to $-78{ }^{\circ} \mathrm{C}$, and ethyl 4,4-difluorocyclohexanecarboxylate (3.67 g, $19.1 \mathrm{mmol}, 1$ equiv) was added dropwise. After stirring the solution for 1 hour, TMS$\mathrm{Cl}$ (2.91 mL, $22.9 \mathrm{mmol}, 1.2$ equiv) was added dropwise. The resulting solution was stirred at this temperature for 30 minutes, and the reaction was then allowed to warm to room temperature where it was stirred for an additional 18 hours. At this time, the reaction was diluted with pentane, and white precipitate was filtered through a $\mathrm{MgSO}_{4}$-packed frit eluting with pentane. All organic solvents were removed in vacuo, and the title compound was isolated via vacuum distillation (b.p. $78^{\circ} \mathrm{C}, 5$ Torr) as a clear colorless liquid (1.63 g, $6.17 \mathrm{mmol}, 32 \%$ yield) ${ }^{\mathbf{1}} \mathbf{H}$ NMR $\left(400 \mathrm{MHz}, \mathrm{CD}_{2} \mathrm{Cl}_{3}\right) \delta 3.77(\mathrm{q}, J=7.1 \mathrm{~Hz}, 2 \mathrm{H}), 2.34-2.27(\mathrm{~m}, 2 \mathrm{H}), 2.25-2.18(\mathrm{~m}, 2 \mathrm{H})$, 
$1.89-1.76(\mathrm{~m}, 4 \mathrm{H}), 1.19(\mathrm{t}, J=7.1 \mathrm{~Hz}, 3 \mathrm{H}), 0.20(\mathrm{~s}, 9 \mathrm{H}) .{ }^{13} \mathbf{C} \mathbf{N M R}\left(100 \mathrm{MHz}, \mathrm{CD}_{2} \mathrm{Cl}_{2}\right) \delta$ $147.8,124.80(\mathrm{t}, J=240.3 \mathrm{~Hz}), 95.4,65.1,35.07(\mathrm{t}, J=22.8 \mathrm{~Hz}), 34.80(\mathrm{t}, J=23.0 \mathrm{~Hz}), 23.46$ $(\mathrm{t}, J=5.4 \mathrm{~Hz}), 23.04(\mathrm{t}, J=5.4 \mathrm{~Hz}), 15.0,0.1(3 \mathrm{C}) .{ }^{19} \mathbf{F} \mathbf{N M R}\left(376 \mathrm{MHz}, \mathrm{CD}_{2} \mathrm{Cl}_{2}\right) \delta-95.3$. IR $\left(\right.$ atr, $\mathrm{cm}^{-1}$ ) 2962, 2900, 2858, 1697, 1447, 1368, 1238, 1189, 1156, 1126, 1100, 1059, 990, 960, 931, 842, 755, 571, 508, 491. HRMS (EI+, M+) Calc'd for $\mathrm{C}_{12} \mathrm{H}_{22} \mathrm{~F}_{2} \mathrm{O}_{2} \mathrm{Si}$ 264.1352, found 264.1353 .

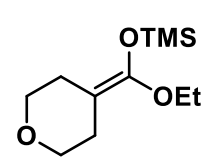

(ethoxy(tetrahydro-4H-pyran-4-ylidene)methoxy)trimethylsilane (2i) - Was synthesized according to general procedure $1 \mathrm{~b}$ using ethyl tetrahydro-2H-pyran4-carboxylate ( $4.00 \mathrm{~g}, 25.3 \mathrm{mmol}, 1$ equiv). The title compound was isolated via vacuum distillation (b.p. $65^{\circ} \mathrm{C}, 5$ Torr) as a clear colorless liquid (1.13 g, 4.91 mmol, $19 \%$ yield) ${ }^{1} \mathbf{H}$ NMR $\left(400 \mathrm{MHz}, \mathrm{CDCl}_{3}\right) \delta 3.79(\mathrm{q}, J=7.1 \mathrm{~Hz}, 2 \mathrm{H}), 3.61(\mathrm{td}, J=5.5,4.4$ $\mathrm{Hz}, 4 \mathrm{H}), 2.28-2.22(\mathrm{~m}, 2 \mathrm{H}), 2.19-2.14(\mathrm{~m}, 2 \mathrm{H}), 1.22(\mathrm{t}, J=7.1 \mathrm{~Hz}, 3 \mathrm{H}), 0.20(\mathrm{~s}, 9 \mathrm{H}) .{ }^{13} \mathbf{C}$ NMR $\left(101 \mathrm{MHz}, \mathrm{CDCl}_{3}\right) \delta 146.8,95.2,68.9,68.6,64.6,27.7,27.4,14.7,-0.0$ (3C). IR (atr, $\mathrm{cm}^{-1}$ ) 2957, 2901, 2839, 1699, 1438, 1335, 1293, 1236, 1180, 1154, 1101, 1065, 1022, 973, 862, 839, 754, 693, 541, 492. HRMS (EI+, M+H) Calc'd for $\mathrm{C}_{11} \mathrm{H}_{22} \mathrm{O}_{3} \mathrm{Si}$ 231.1411, found 231.1413 .

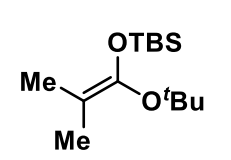

((1-(tert-butoxy)-2-methylprop-1-en-1-yl)oxy)(tert-butyl)dimethylsilane (2j) - Was synthesized according to general procedure 1a using tert-butyl isobutyrate (6.06 g, $42 \mathrm{mmol}, 1$ equiv). The title compound was isolated via vacuum distillation (b.p. $65^{\circ} \mathrm{C}, 5$ Torr) as a clear colorless liquid $(8.51 \mathrm{~g}, 32.9 \mathrm{mmol}$, $78 \%$ yield) ${ }^{1} \mathbf{H}$ NMR (400 MHz, $\left.\mathrm{CDCl}_{3}\right) \delta 1.57(\mathrm{~s}, 3 \mathrm{H}), 1.54(\mathrm{~s}, 3 \mathrm{H}), 1.28(\mathrm{~s}, 9 \mathrm{H}), 0.95(\mathrm{~s}, 9 \mathrm{H})$, 0.13 (s, 6H). ${ }^{13} \mathrm{C} \mathrm{NMR}\left(100 \mathrm{MHz}, \mathrm{CDCl}_{3}\right) \delta 146.5,96.2,79.2,29.1$ (3C), $25.8(3 \mathrm{C}), 18.3,17.9$, 17.6, -4.0 (2C). IR (atr, $\mathrm{cm}^{-1}$ ) 2977, 2930, 2859, 1691, 1473, 1389, 1365, 1251, 1201, 1136, 988, 853, 837, 779, 678, 556, 511. HRMS (EI+, M-[C $\left.\mathrm{C}_{4} \mathrm{H}_{8}\right]$ ) Calc'd for $\mathrm{C}_{10} \mathrm{H}_{22} \mathrm{O}_{2} \mathrm{Si} 202.13836$, found 202.13834 .<smiles>CCOC=CO</smiles>

tert-butyl((1-ethoxyvinyl)oxy)dimethylsilane (2k) - Was synthesized according to general procedure 1a using ethyl acetate $(4.88 \mathrm{~mL}, 50 \mathrm{mmol}, 1$ equiv). The title compound was isolated via vacuum distillation (b.p. $75{ }^{\circ} \mathrm{C}, 15$ mbar) as a clear colorless liquid $(8.91 \mathrm{~g}, 44.0 \mathrm{mmol}, 88 \%$ yield $)$. Spectral data was in accordance with the reported literature. ${ }^{16}{ }^{1} \mathbf{H}$ NMR $\left(400 \mathrm{MHz}, \mathrm{CDCl}_{3}\right) \delta 3.75(\mathrm{q}, J=7.0 \mathrm{~Hz}, 2 \mathrm{H}), 3.22(\mathrm{~d}$, $J=2.4 \mathrm{~Hz}, 1 \mathrm{H}), 3.06(\mathrm{~d}, J=2.4 \mathrm{~Hz}, 1 \mathrm{H}), 1.29(\mathrm{t}, J=7.0 \mathrm{~Hz}, 3 \mathrm{H}), 0.93(\mathrm{~s}, 9 \mathrm{H}), 0.18(\mathrm{~s}, 6 \mathrm{H})$. ${ }^{13} \mathrm{C}$ NMR $\left(100 \mathrm{MHz}, \mathrm{CDCl}_{3}\right) \delta 161.2,63.3,60.4,25.7$ (3C), 18.2, 14.4, -4.5 (2C). 
General procedure 2: Enantioselective Ir-catalyzed allenylic alkylation.

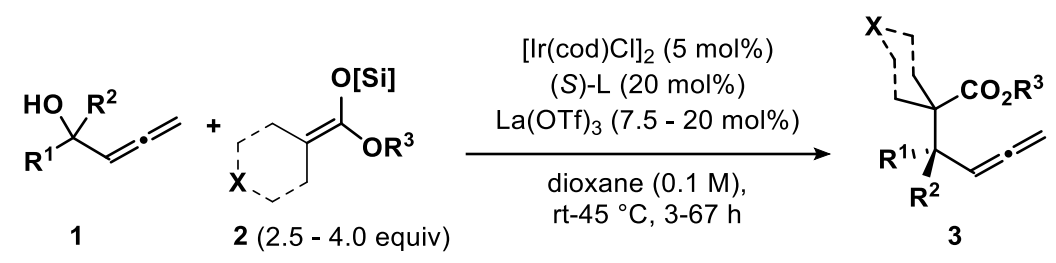

General Procedure 2a - solid substrates: A dry $5 \mathrm{~mL}$ vial containing a magnetic stir-bar was charged with ( \pm -1 $\mathbf{1}(0.4 \mathrm{mmol}, 1$ equiv $),[\operatorname{Ir}(\operatorname{cod}) \mathrm{Cl}]_{2}(20 \mu \mathrm{mol}, 5.0 \mathrm{~mol} \%)$ and $(S)-\mathbf{L} \mathbf{1}(80 \mu \mathrm{mol}$, $20 \mathrm{~mol} \%)$. The solids were taken up in 1,4-dioxane $(4.0 \mathrm{~mL},[( \pm)-1]=0.1 \mathrm{M})$ and the resulting solution was stirred under dry Ar gas for 10 minutes. Silyl ketene acetal 2 (1.0 mmol, 2.5 equiv) and $\mathrm{La}(\mathrm{OTf})_{3}(30 \mu \mathrm{mol}, 7.5 \mathrm{~mol} \%)$ were then added, and the vial was re-purged with Ar and then sealed with a PTFE screw cap. The reaction was stirred at $45{ }^{\circ} \mathrm{C}$ for the indicated time and then the reaction mixture was passed through the plug of Celite eluting with $\mathrm{Et}_{2} \mathrm{O}$ and the filtrate was concentrated in vасио. The crude residue was purified via flash column chromatography using silica gel with the indicated mobile phase.

General Procedure 2b - liquid substrates: A dry $5 \mathrm{~mL}$ vial containing a magnetic stir-bar was charged with $( \pm)-\mathbf{1}(0.4 \mathrm{mmol}, 1$ equiv), which was dissolved in 1,4-dioxane $(4.0 \mathrm{~mL},[( \pm)-\mathbf{1}]=$ $0.1 \mathrm{M})$. $[\mathrm{Ir}(\mathrm{cod}) \mathrm{Cl}]_{2}(20 \mu \mathrm{mol}, 5.0 \mathrm{~mol} \%)$ and $(S)-\mathbf{L}_{1}(80 \mu \mathrm{mol}, 20 \mathrm{~mol} \%)$ were added and the resulting solution was stirred under dry Ar gas for 10 minutes. Silyl ketene acetal $2(1.0 \mathrm{mmol}$, 2.5 equiv) and $\mathrm{La}(\mathrm{OTf})_{3}(30 \mu \mathrm{mol}, 7.5 \mathrm{~mol} \%)$ were then added, and the vial was re-purged with Ar and then sealed with a PTFE screw cap. The reaction was stirred at $45{ }^{\circ} \mathrm{C}$ for the indicated time and then the reaction mixture was passed through the plug of Celite eluting with $\mathrm{Et}_{2} \mathrm{O}$ and the filtrate was concentrated in vacuo. The crude residue was purified via flash column chromatography using silica gel with the indicated mobile phase.

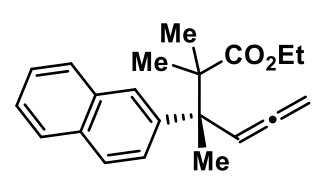

ethyl $(R)$-2,2,3-trimethyl-3-(naphthalen-2-yl)hexa-4,5-dienoate (3a) Was synthesized according to general procedure 2 a using $1 \mathbf{a}(84 \mathrm{mg}, 0.4$ $\mathrm{mmol}),[\mathrm{Ir}(\mathrm{cod}) \mathrm{Cl}]_{2}(13.4 \mathrm{mg}, 20 \mu \mathrm{mol}, 5.0 \mathrm{~mol} \%),(S)-\mathbf{L} \mathbf{1}(40.6 \mathrm{mg}, 80$ $\mu \mathrm{mol}, 20 \mathrm{~mol} \%), \mathbf{2 a}(246 \mu \mathrm{L}, 1.0 \mathrm{mmol}, 2.5 \text { equiv), and La(OTf })_{3}(17.6$ $\mathrm{mg}, 30 \mu \mathrm{mol}, 7.5 \mathrm{~mol} \%$ ), and the reaction was run for 17 hours. The regiomeric ratio (rr) was determined to be $>20: 1$ by ${ }^{1} \mathrm{H}$ NMR analysis of the unpurified reaction mixture. The crude residue was purified via $\mathrm{SiO}_{2}$ flash column chromatography using pentane: $\mathrm{Et}_{2} \mathrm{O}$ (100:1 to 50:1 $\mathrm{v}: \mathrm{v})$. The title compound was obtained as white solid $(92 \mathrm{mg}, 0.30 \mathrm{mmol}, 75 \%$ yield, $\mathrm{MP}=$ 57.5-58.1 $\left.{ }^{\circ} \mathrm{C}\right) .{ }^{1} \mathbf{H}$ NMR $\left(400 \mathrm{MHz}, \mathrm{CDCl}_{3}\right) \delta 7.85-7.77(\mathrm{~m}, 3 \mathrm{H}), 7.77-7.72(\mathrm{~m}, 1 \mathrm{H}), 7.52$ $(\mathrm{dd}, J=8.8,2.1 \mathrm{~Hz}, 1 \mathrm{H}), 7.49-7.40(\mathrm{~m}, 2 \mathrm{H}), 6.26(\mathrm{t}, J=6.7 \mathrm{~Hz}, 1 \mathrm{H}), 4.82-4.65(\mathrm{~m}, 2 \mathrm{H})$, $3.97(\mathrm{q}, J=7.1 \mathrm{~Hz}, 2 \mathrm{H}), 1.67(\mathrm{~s}, 3 \mathrm{H}), 1.20(\mathrm{~s}, 6 \mathrm{H}), 1.03(\mathrm{t}, J=7.1 \mathrm{~Hz}, 3 \mathrm{H}) .{ }^{13} \mathbf{C}$ NMR $(100$ $\left.\mathrm{MHz}, \mathrm{CDCl}_{3}\right) \delta 207.7,176.3,142.1,132.7,131.9,128.1,127.2,126.8,126.6,126.4,125.8$, 125.7, 96.8, 77.0, 60.3, 49.6, 46.5, 22.4, 22.3, 22.1, 13.9. IR (atr, $\left.\mathrm{cm}^{-1}\right)$ 3057, 2979, 2948, 1954, $1717,1600,1465,1381,1265,1139,1087,1026,856,818,745,477$. HRMS (ESI+, M+Na) Calc'd for $\mathrm{C}_{21} \mathrm{H}_{24} \mathrm{O}_{2} \mathrm{Na} 331.1669$, found 331.1669 . Enantiomeric purity was determined by HPLC analysis in comparison with racemic material $T_{R}=6.7$ (minor) $T_{R}=7.9$ (major) (99.5:0.5 er shown); Chiracel IC column, $98 \%$ hexane to $2 \% \mathrm{iPrOH}, 1.0 \mathrm{~mL} / \mathrm{min}, 222 \mathrm{~nm}, 480 \mathrm{psi}, 26$ $\left.{ }^{\circ} \mathrm{C}\right)[\alpha]_{D}^{23}=-173.27\left(\mathrm{c}=0.5, \mathrm{CHCl}_{3}\right)$. The absolute stereochemistry of this compound was assigned by X-ray crystallographic analysis of a derivative $(R)$-S5 (vide infra). 

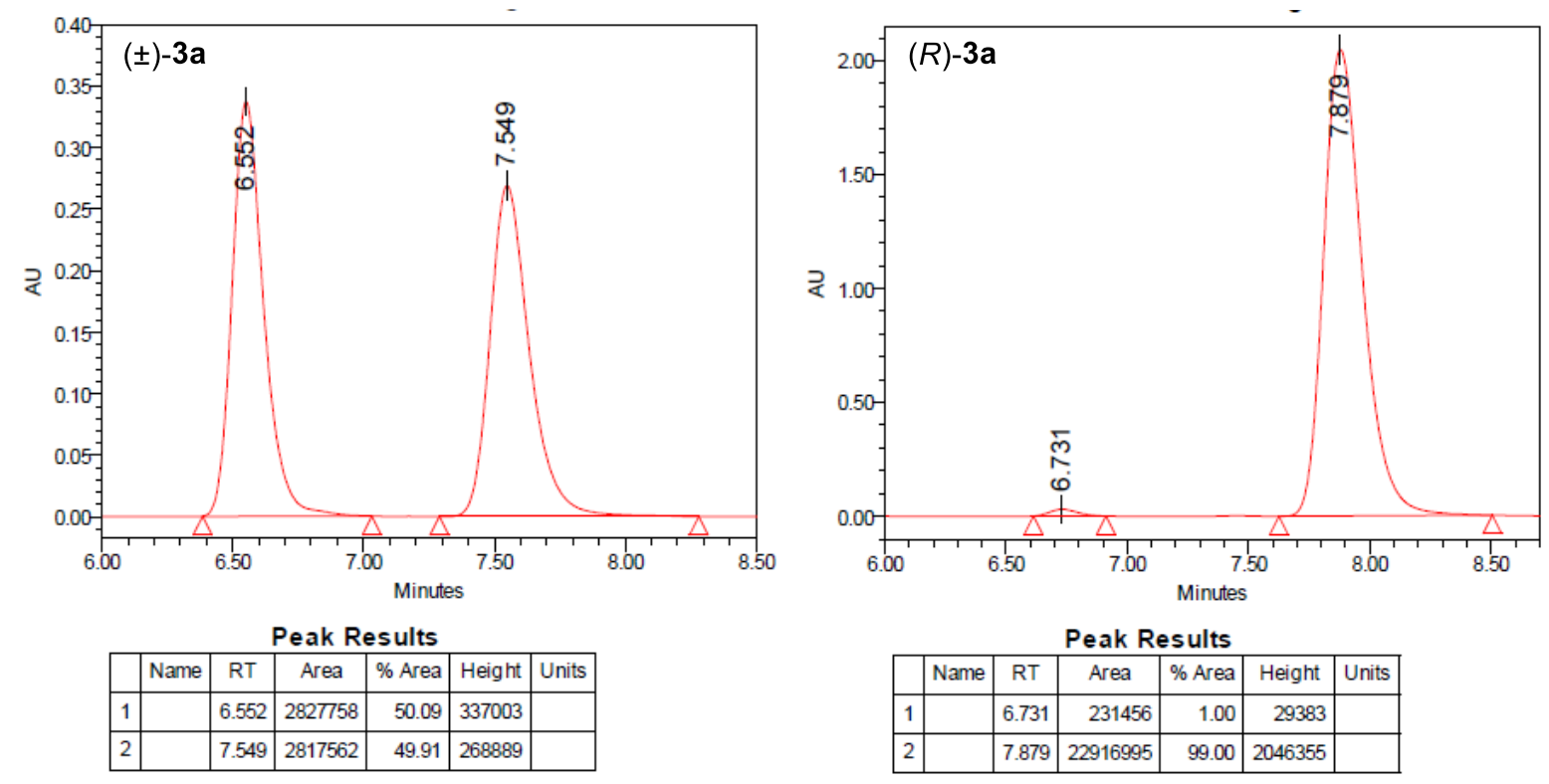

\section{Experimental procedures for the gram-scale asymmetric alkylation:}

A dry $50 \mathrm{~mL}$ Schott bottle containing a magnetic stir-bar was charged with $( \pm)-1 \mathrm{a}(1.0 \mathrm{~g}, 4.8$ mmol, 1 equiv), [ $\operatorname{Ir}(\mathrm{cod}) \mathrm{Cl}]_{2}(160 \mathrm{mg}, 0.24 \mathrm{mmol}, 5.0 \mathrm{~mol} \%)$, and $(S)-\mathbf{L}_{\mathbf{1}}(483 \mathrm{mg}, 0.95 \mathrm{mmol}$, $20 \mathrm{~mol} \%)$. The solids were taken up with 1,4 -dioxane $(47.6 \mathrm{~mL},[( \pm)-1 \mathrm{a}]=0.1 \mathrm{M})$ and the resulting solution was stirred under dry Ar gas for 10 minutes. Silyl ketene acetal $\mathbf{2 a}(3.15 \mathrm{~mL}$, $11.9 \mathrm{mmol}, 2.5$ equiv), and $\mathrm{La}(\mathrm{OTf})_{3}(209 \mathrm{mg}, 0.36 \mathrm{mmol}, 7.5 \mathrm{~mol} \%)$ were then added in that order, and the reaction vessel was re-purged with dry Ar gas and sealed with the matching Schott cap. The reaction mixture was stirred at $45^{\circ} \mathrm{C}$ for 17 hours, at which time the solution was filtered through a pad of Celite eluting with $\mathrm{Et}_{2} \mathrm{O}(75 \mathrm{~mL})$ and the filtrate was concentrated in vасио. The crude residue was purified via $\mathrm{SiO}_{2}$ flash column chromatography using pentane:Et $2 \mathrm{O}$ (100:1 to 50:1 v:v). The product was obtained as a white solid $(1.1 \mathrm{~g}, 3.6 \mathrm{mmol}$, $76 \%$ yield). Enantiomeric purity was determined by HPLC analysis in comparison with racemic material $\mathrm{T}_{\mathrm{R}}=5.7$ (minor) $\mathrm{T}_{\mathrm{R}}=6.4$ (major) (99.5:0.5 er shown); Chiracel IC column, 98\% hexane to $2 \% \mathrm{iPrOH}, 1.0 \mathrm{~mL} / \mathrm{min}, 220 \mathrm{~nm}, 480 \mathrm{psi}, 25^{\circ} \mathrm{C}$ )
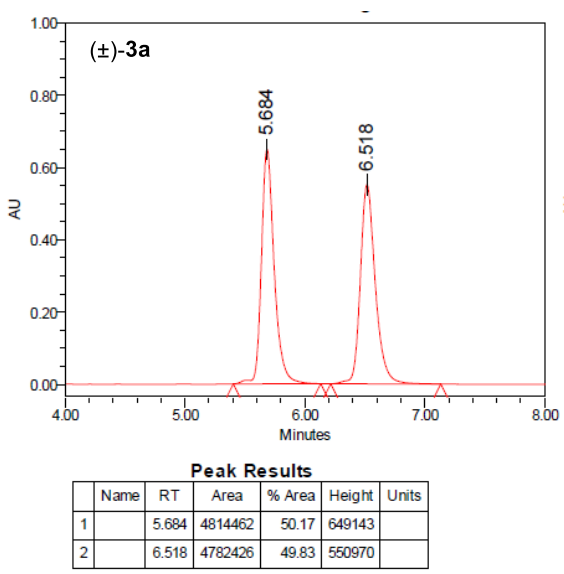
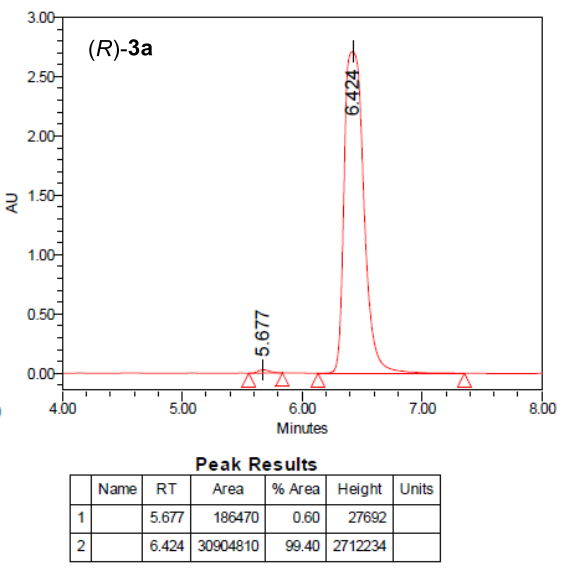

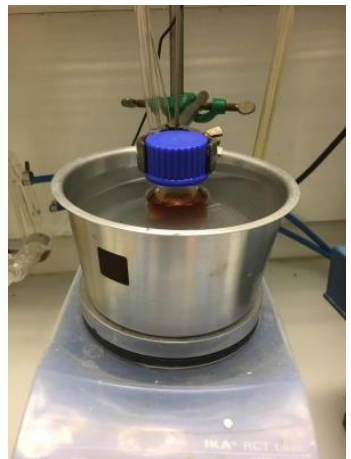

Figure S1. Gram scale experiment of allenylic alkylation reaction. 
<smiles>C=CC(=O)C(C)(C)C(C)(C)C(C)(C)OCC</smiles>

ethyl $(R)-3-(6-b r o m o n a p h t h a l e n-2-y l)-2,2,3-t r i m e t h y l h e x a-4,5-$

dienoate $(3 \mathbf{b})$ - Was synthesized according to general procedure $2 \mathrm{a}$ using $1 \mathbf{b}(116 \mathrm{mg}, 0.4 \mathrm{mmol})$, [ $[\mathrm{Ir}(\operatorname{cod}) \mathrm{Cl}]_{2}(13.4 \mathrm{mg}, 20 \mu \mathrm{mol}, 5.0$ mol\%), (S)-L1 (40.6 mg, $80 \mu \mathrm{mol}, 20 \mathrm{~mol} \%), \mathbf{2 b}(222 \mu \mathrm{L}, 1.0 \mathrm{mmol}$, 2.5 equiv), and $\mathrm{La}(\mathrm{OTf})_{3}(17.6 \mathrm{mg}, 30 \mu \mathrm{mol}, 7.5 \mathrm{~mol} \%)$, and the reaction was run for 20 hours. The regiomeric ratio (rr) was determined to be $>20: 1$ by ${ }^{1} \mathrm{H}$ NMR analysis of the unpurified reaction mixture. The title compound was obtained by purification via $\mathrm{SiO}_{2}$ flash column chromatography using pentane:Et $\mathrm{t}_{2} \mathrm{O}(35: 1 \mathrm{v}: \mathrm{v})$. The title compound was obtained as a slightly yellow oil (119.4 mg, $0.31 \mathrm{mmol}, 77 \%$ yield). ${ }^{1} \mathbf{H} \mathbf{~ N M R}\left(400 \mathrm{MHz}, \mathrm{CDCl}_{3}\right) \delta 7.98-7.94(\mathrm{~m}$, $1 \mathrm{H}), 7.79-7.74(\mathrm{~m}, 1 \mathrm{H}), 7.70-7.61(\mathrm{~m}, 2 \mathrm{H}), 7.58-7.48(\mathrm{~m}, 2 \mathrm{H}), 6.23(\mathrm{t}, J=6.7 \mathrm{~Hz}, 1 \mathrm{H})$, $4.84-4.67(\mathrm{~m}, 2 \mathrm{H}), 3.95(\mathrm{q}, J=7.1 \mathrm{~Hz}, 2 \mathrm{H}), 1.65(\mathrm{~s}, 3 \mathrm{H}), 1.19(\mathrm{~s}, 6 \mathrm{H}), 1.03$ (t, $J=7.1 \mathrm{~Hz}$, $3 \mathrm{H}) .{ }^{13} \mathrm{C}$ NMR $\left(100 \mathrm{MHz}, \mathrm{CDCl}_{3}\right) \delta 207.7,176.1,142.8,132.9,131.1,129.8,129.2,129.2$, 127.7, 126.7, 125.5, 119.6, 96.6, 77.1, 60.3, 49.6, 46.5, 22.4, 22.2, 22.1, 13.9. IR (atr, $\left.\mathrm{cm}^{-1}\right)$ 3057, 2978, 1954, 1714, 1583, 1494, 1461, 1380, 1262, 1170, 1129, 1085, 1059, 1024, 950, 876, 841, 805, 712, 669, 474. HRMS (ESI+, M+Na) Calc'd for $\mathrm{C}_{21} \mathrm{H}_{23} \mathrm{O}_{2} \mathrm{BrNa}$ 409.0774, found 409.0771. Enantiomeric purity was determined by HPLC analysis in comparison with racemic material $\mathrm{T}_{\mathrm{R}}=5.9$ (minor) $\mathrm{T}_{\mathrm{R}}=6.6$ (major) (96:4 er shown); Chiracel IC column, 99\% hexane to $\left.1 \% \mathrm{iPrOH}, 1.0 \mathrm{~mL} / \mathrm{min}, 231 \mathrm{~nm}, 510 \mathrm{psi}, 26{ }^{\circ} \mathrm{C}\right)[\boldsymbol{\alpha}]_{\boldsymbol{D}}^{\mathbf{2 6}}=-157.5\left(\mathrm{c}=0.5, \mathrm{CHCl}_{3}\right)$. The absolute stereochemistry of this compound was assigned in analogy to $\mathbf{3 a}$.
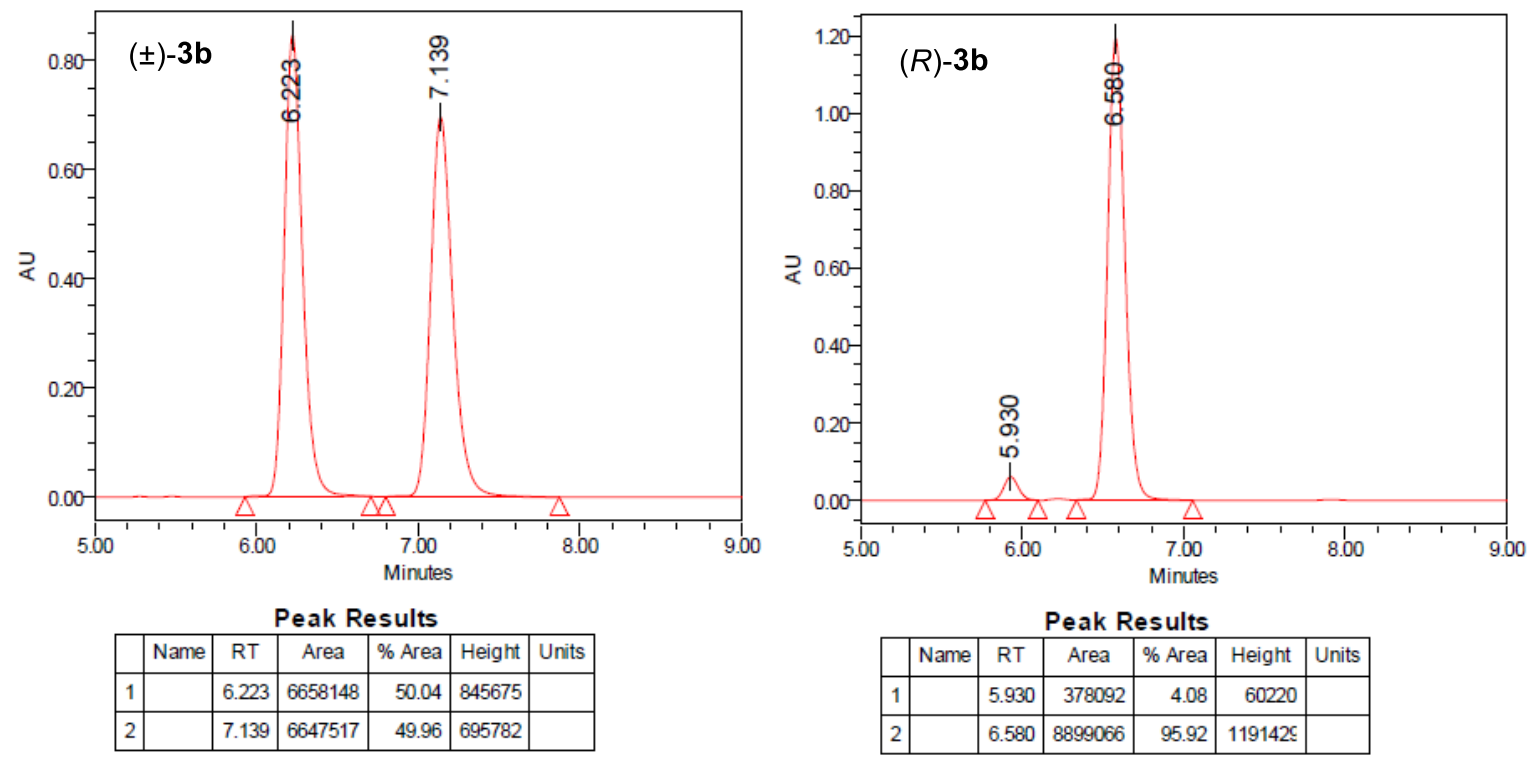


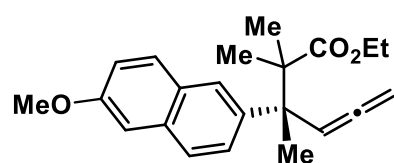

ethyl (R)-3-(6-methoxynaphthalen-2-yl)-2,2,3-trimethylhexa-4,5dienoate $(3 \mathbf{c})$ - Was synthesized according to general procedure $2 \mathrm{a}$ using 1c $(84 \mathrm{mg}, 0.4 \mathrm{mmol}),[\operatorname{Ir}(\operatorname{cod}) \mathrm{Cl}]_{2}(13.4 \mathrm{mg}, 20 \mu \mathrm{mol}, 5.0$ mol\%), (S)-L1 (40.6 mg, $80 \mu \mathrm{mol}, 20 \mathrm{~mol} \%)$, 2a (246 $\mu \mathrm{L}, 1.0 \mathrm{mmol}$, 2.5 equiv), and $\mathrm{La}(\mathrm{OTf})_{3}(17.6 \mathrm{mg}, 30 \mu \mathrm{mol}, 7.5 \mathrm{~mol} \%)$, and the reaction was run for 7 hours. The regiomeric ratio (rr) was determined to be $>20: 1$ by ${ }^{1} \mathrm{H}$ NMR analysis of the unpurified reaction mixture. The title compound was obtained by purification via $\mathrm{SiO}_{2}$ flash column chromatography using pentane: $\mathrm{Et}_{2} \mathrm{O}(50: 1$ to $20: 1 \mathrm{v}: \mathrm{v})$. The title compound was obtained as white solid (98.6 mg, $0.29 \mathrm{mmol}, 73 \%$ yield, $\left.\mathrm{MP}=68.0-68.7^{\circ} \mathrm{C}\right) .{ }^{1} \mathbf{H} \mathbf{~ N M R}\left(400 \mathrm{MHz}, \mathrm{CDCl}_{3}\right)$ $\delta 7.77-7.62(\mathrm{~m}, 3 \mathrm{H}), 7.50(\mathrm{dd}, J=8.7,1.7 \mathrm{~Hz}, 1 \mathrm{H}), 7.17-7.07(\mathrm{~m}, 2 \mathrm{H}), 6.26(\mathrm{t}, J=6.6 \mathrm{~Hz}$, $1 \mathrm{H}), 4.83-4.68(\mathrm{~m}, 2 \mathrm{H}), 3.98(\mathrm{~d}, J=7.1 \mathrm{~Hz}, 2 \mathrm{H}), 3.92(\mathrm{~s}, 3 \mathrm{H}), 1.67(\mathrm{~s}, 3 \mathrm{H}), 1.21(\mathrm{~s}, 6 \mathrm{H}), 1.05$ $(\mathrm{t}, J=7.1 \mathrm{~Hz}, 3 \mathrm{H}) .{ }^{13} \mathbf{C}$ NMR $\left(100 \mathrm{MHz}, \mathrm{CDCl}_{3}\right) \delta 207.7,176.3,157.6,139.8,133.0,129.6$, 128.2, 127.1, 126.6, 125.3, 118.6, 105.1, 96.8, 76.9, 60.3, 55.3, 49.7, 46.3, 22.4, 22.3, 22.1, 13.9. IR $\left(\right.$ atr, $\left.\mathrm{cm}^{-1}\right) 2970,2935,1950,1725,1602,1629,1452,1389,1258,1223,1161,1132$, $1083,1022,925,850,764,734,676,523,476$. HRMS (ESI+, M+Na) Calc'd for $\mathrm{C}_{22} \mathrm{H}_{26} \mathrm{O}_{3} \mathrm{Na}$ 361.1774, found 361.1779. Enantiomeric purity was determined by HPLC analysis in comparison with racemic material $\mathrm{T}_{\mathrm{R}}=8.9$ (minor) $\mathrm{T}_{\mathrm{R}}=10.5$ (major) (98.5:1.5 er shown); Chiralcel IC column, $98 \%$ hexane to $\left.2 \% \mathrm{iPrOH}, 1.0 \mathrm{~mL} / \mathrm{min}, 234 \mathrm{~nm}, 540 \mathrm{psi}, 26^{\circ} \mathrm{C}\right)[\boldsymbol{\alpha}]_{D}^{25}=$ $-155.6\left(\mathrm{c}=0.5, \mathrm{CHCl}_{3}\right)$. The absolute stereochemistry of this compound was assigned in analogy to $3 \mathbf{a}$.
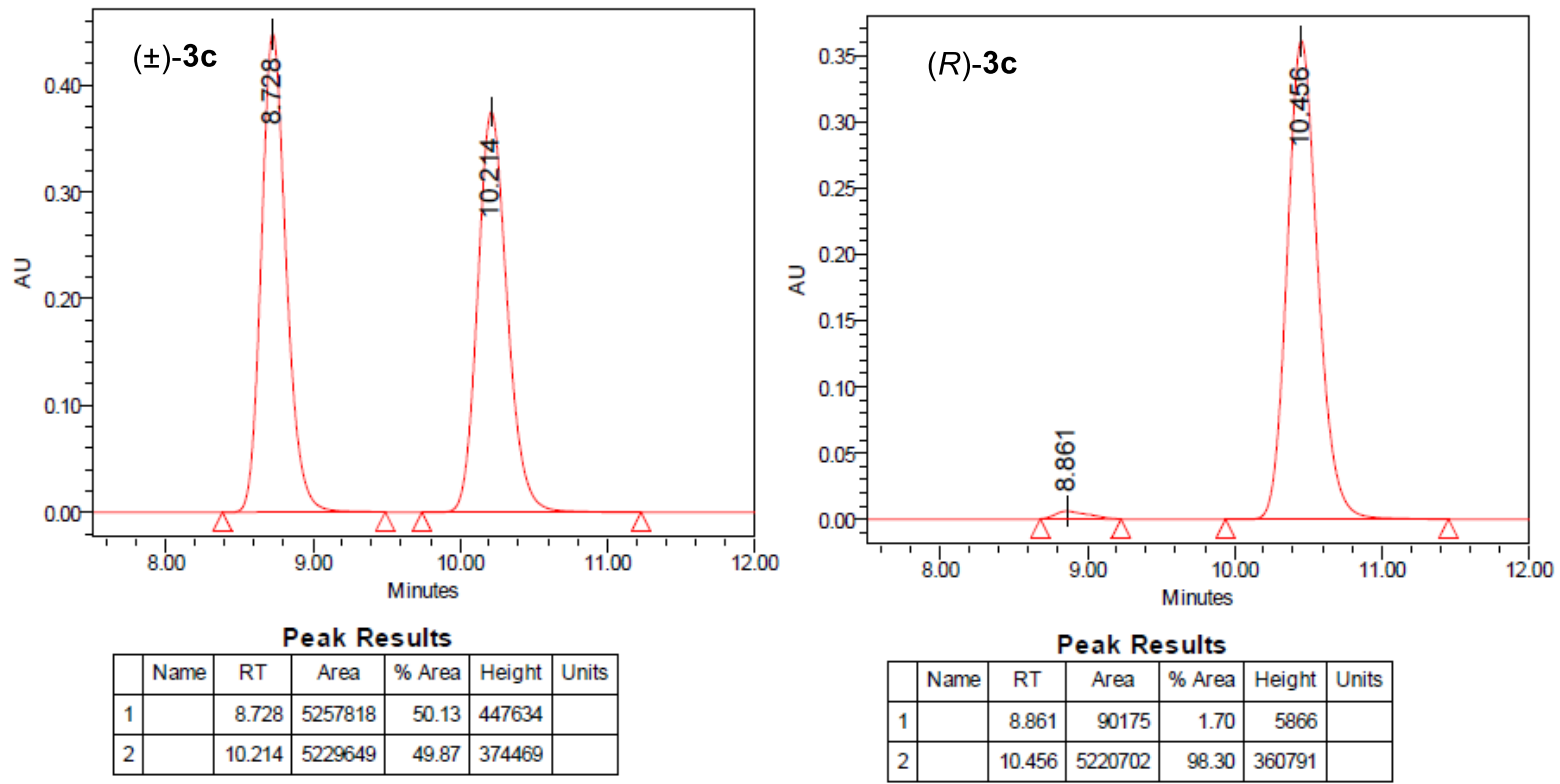


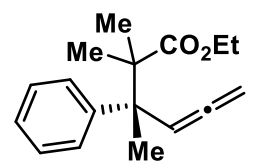

ethyl $(\boldsymbol{R})-2,2,3-$ trimethyl-3-phenylhexa-4,5-dienoate $(\mathbf{3 d}) \quad-\quad$ Was synthesized according to general procedure $2 \mathrm{~b}$ using $1 \mathrm{~d}(64.1 \mathrm{mg}, 0.4 \mathrm{mmol})$, $[\operatorname{Ir}(\operatorname{cod}) \mathrm{Cl}]_{2}(13.4 \mathrm{mg}, 20 \mu \mathrm{mol}, 5.0 \mathrm{~mol} \%),(S)-\mathbf{L} \mathbf{1}(40.6 \mathrm{mg}, 80 \mu \mathrm{mol}, 20$ mol\%), $2 \mathbf{a}(265 \mu \mathrm{L}, 1.0 \mathrm{mmol}, 2.5$ equiv), and La(OTf) $3(17.6 \mathrm{mg}, 30 \mu \mathrm{mol}$, $7.5 \mathrm{~mol} \%$ ), and the reaction was run for 20 hours. The regiomeric ratio (rr) was determined to be $>20: 1$ by ${ }^{1} \mathrm{H}$ NMR analysis of the unpurified reaction mixture. The title compound was obtained by purification via $\mathrm{SiO}_{2}$ flash column chromatography using pentane:Et ${ }_{2} \mathrm{O}(50: 1 \mathrm{v}: \mathrm{v})$. The title compound was obtained as a clear colorless oil $(67.5 \mathrm{mg}, 0.26 \mathrm{mmol}, 65 \%$ yield $) .{ }^{1} \mathbf{H}$ NMR $\left(400 \mathrm{MHz}, \mathrm{CDCl}_{3}\right) \delta 7.38-7.33(\mathrm{~m}, 2 \mathrm{H}), 7.30-7.24(\mathrm{~m}, 2 \mathrm{H}), 7.23-7.16(\mathrm{~m}, 1 \mathrm{H}), 6.17$ $-6.11(\mathrm{~m}, 1 \mathrm{H}), 4.82-4.66(\mathrm{~m}, 2 \mathrm{H}), 3.97(\mathrm{q}, J=7.1 \mathrm{~Hz}, 2 \mathrm{H}), 1.56(\mathrm{~s}, 3 \mathrm{H}), 1.15(\mathrm{~s}, 6 \mathrm{H}), 1.11$ $(\mathrm{t}, J=7.1 \mathrm{~Hz}, 3 \mathrm{H}) .{ }^{13} \mathrm{C}$ NMR $\left(100 \mathrm{MHz}, \mathrm{CDCl}_{3}\right) \delta 207.6,176.2,144.5,128.0(2 \mathrm{C}), 127.3(2 \mathrm{C})$, 126.3, 96.7, 76.9, 60.3, 49.5, 46.2, 22.3, 22.1, 21.8, 13.9.IR (atr, $\left.\mathrm{cm}^{-1}\right)$ 2979, 2938, 1955, 1715, 1599, 1494, 1464, 1444, 1380, 1263, 1193, 1171, 1136, 1082, 1027, 839, 758, 699, 661, 524. HRMS (ESI+, M+Na) Calc'd for $\mathrm{C}_{17} \mathrm{H}_{22} \mathrm{O}_{2} \mathrm{Na} 281.1512$, found 281.1513. Enantiomeric purity was determined by SFC analysis in comparison with racemic material $\mathrm{T}_{\mathrm{R}}=4.1$ (minor) $\mathrm{T}_{\mathrm{R}}=$ 4.6 (major) (98:2 er shown); Chiracel OJ-H column, 95\% $\mathrm{CO}_{2}$ to $5 \% \mathrm{MeOH}, 2.0 \mathrm{~mL} / \mathrm{min}, 202$ $\mathrm{nm}, 127$ bar, $\left.25{ }^{\circ} \mathrm{C}\right) .[\boldsymbol{\alpha}]_{\boldsymbol{D}}^{\mathbf{2 4}}=-61.4\left(\mathrm{c}=0.5, \mathrm{CHCl}_{3}\right)$. The absolute stereochemistry of this compound was assigned in analogy to $\mathbf{3 a}$.
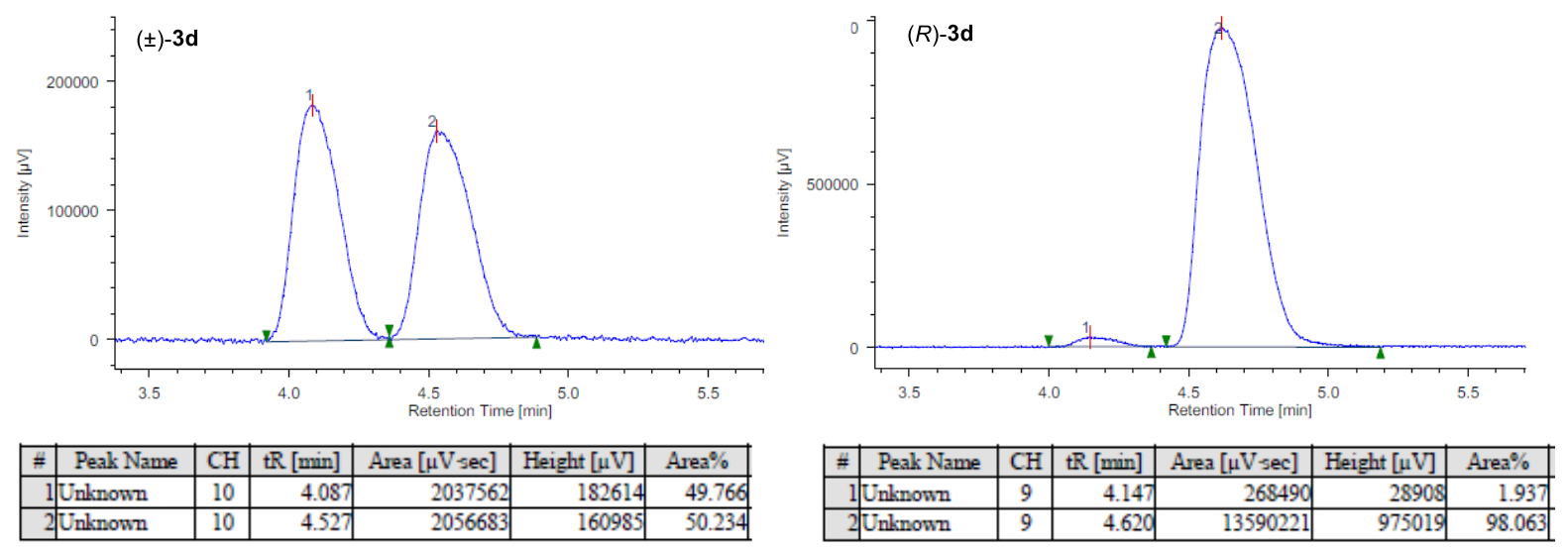


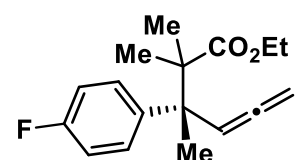

ethyl $(R)$-3-(4-fluorophenyl)-2,2,3-trimethylhexa-4,5-dienoate (3e) Was synthesized according to general procedure $2 \mathrm{~b}$ using $1 \mathrm{e}(71.3 \mathrm{mg}, 0.4$ mmol), $[\operatorname{Ir}(\operatorname{cod}) \mathrm{Cl}]_{2}(13.4 \mathrm{mg}, 20 \mu \mathrm{mol}, 5.0 \mathrm{~mol} \%),(S)-\mathbf{L} 1$ (40.6 mg, 80 $\mu \mathrm{mol}, 20 \mathrm{~mol} \%), \mathbf{2 b}\left(222 \mu \mathrm{L}, 1.0 \mathrm{mmol}, 2.5\right.$ equiv), and $\mathrm{La}(\mathrm{OTf})_{3}(17.6$ $\mathrm{mg}, 30 \mu \mathrm{mol}, 7.5 \mathrm{~mol} \%$ ), and the reaction was run for 14 hours. The regiomeric ratio (rr) was determined to be $>20: 1$ by ${ }^{1} \mathrm{H}$ NMR analysis of the unpurified reaction mixture. The title compound was obtained by purification via $\mathrm{SiO}_{2}$ flash column chromatography using $100 \%$ pentane to pentane:Et ${ }_{2} \mathrm{O}(50: 1 \mathrm{v}: \mathrm{v})$. The title compound was obtained as a clear colorless oil (84.3 mg, $0.31 \mathrm{mmol}, 76 \%$ yield). ${ }^{1} \mathbf{H}$ NMR $\left(400 \mathrm{MHz}, \mathrm{CDCl}_{3}\right) \delta 7.35-7.27$ (m, 2H), $7.01-$ $6.91(\mathrm{~m}, 2 \mathrm{H}), 6.09(\mathrm{t}, J=6.7 \mathrm{~Hz}, 1 \mathrm{H}), 4.82-4.67(\mathrm{~m}, 2 \mathrm{H}), 3.98(\mathrm{q}, J=7.1 \mathrm{~Hz}, 2 \mathrm{H}), 1.54(\mathrm{~s}$, $3 \mathrm{H}), 1.15(\mathrm{~s}, 3 \mathrm{H}), 1.14(\mathrm{~s}, 3 \mathrm{H}), 1.12(\mathrm{~d}, J=7.1 \mathrm{~Hz}, 3 \mathrm{H}) .{ }^{13} \mathbf{C} \mathbf{N M R}\left(100 \mathrm{MHz}, \mathrm{CDCl}_{3}\right) \delta$ 207.6, $176.1,161.3(\mathrm{~d}, J=245.4 \mathrm{~Hz}), 140.2(\mathrm{~d}, J=3.4 \mathrm{~Hz}), 129.6,129.6,114.0,113.8,96.6,77.1$, $60.3,49.5(\mathrm{~d}, J=1.1 \mathrm{~Hz}), 45.9,22.3,22.2,22.1,13.9 .{ }^{19} \mathbf{F}$ NMR $\left(377 \mathrm{MHz}, \mathrm{CDCl}_{3}\right) \delta$-117.2. IR $\left(\operatorname{atr}, \mathrm{cm}^{-1}\right)$ 2980, 1955, 1717, 1601, 1510, 1472, 1380, 1264, 1237, 1167, 1139, 1085, 1026, 843, 823, 599, 536. HRMS (ESI+, M+Na) Calc'd for $\mathrm{C}_{17} \mathrm{H}_{21} \mathrm{O}_{2} \mathrm{FNa} 299.1418$, found 299.1419 . Enantiomeric purity was determined by HPLC analysis in comparison with racemic material $\mathrm{T}_{\mathrm{R}}=6.9$ (minor) $\mathrm{T}_{\mathrm{R}}=7.9$ (major) (96:4 er shown); Chiracel IC column, $99.7 \%$ hexane to $0.3 \%$ iPrOH, $\left.1.0 \mathrm{~mL} / \mathrm{min}, 209 \mathrm{~nm}, 485 \mathrm{psi}, 25{ }^{\circ} \mathrm{C}\right)[\boldsymbol{\alpha}]_{\boldsymbol{D}}^{\mathbf{2 4}}=-66.4\left(\mathrm{c}=0.5, \mathrm{CHCl}_{3}\right)$. The absolute stereochemistry of this compound was assigned in analogy to $\mathbf{3 a}$.
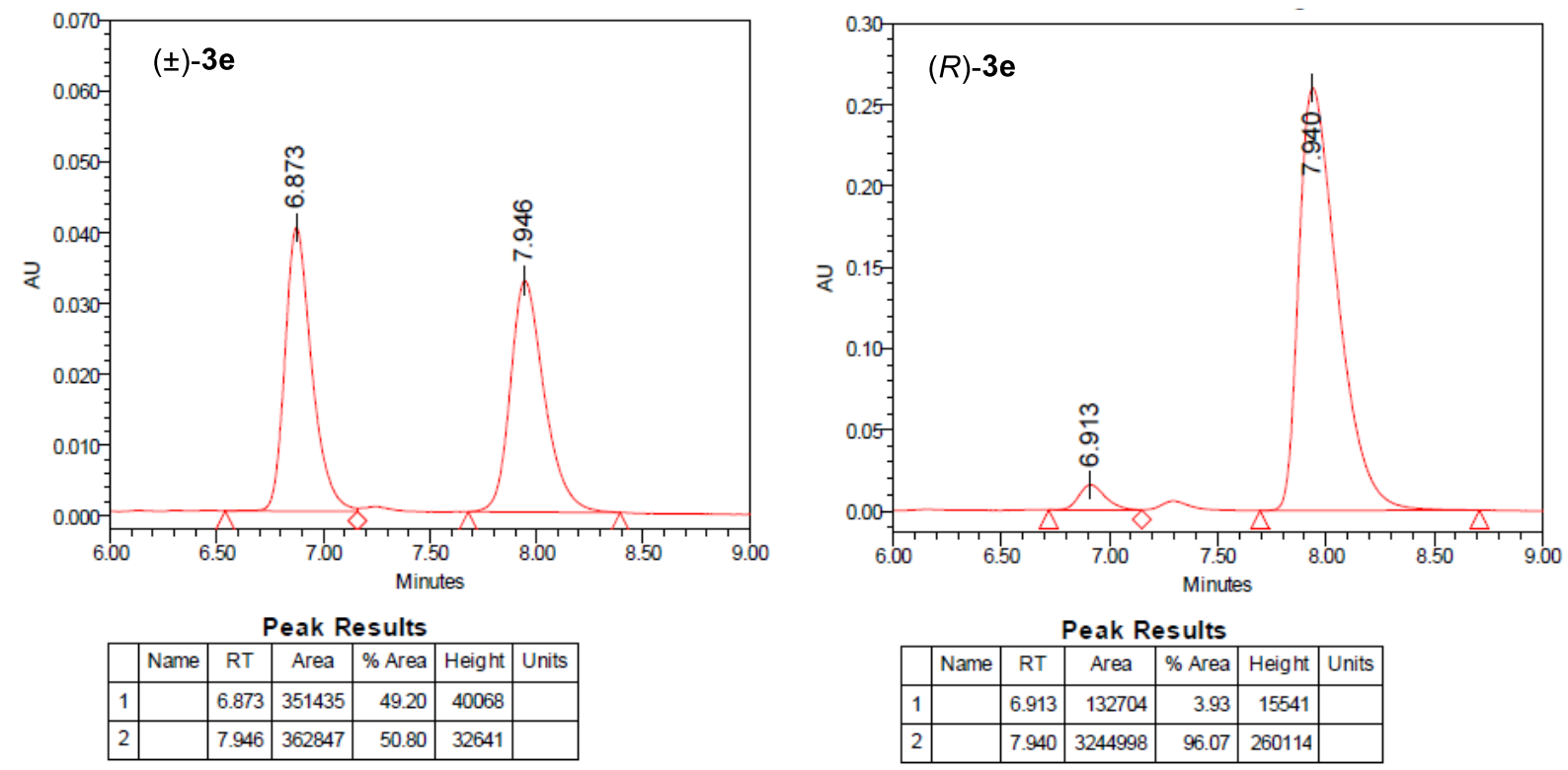


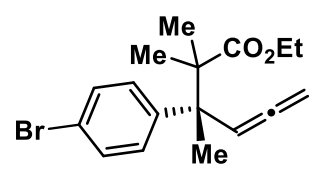

ethyl (R)-3-(4-bromophenyl)-2,2,3-trimethylhexa-4,5-dienoate (3f) Was synthesized according to general procedure 2 a using $\mathbf{1 f}(95.6 \mathrm{mg}, 0.4$ $\mathrm{mmol}),[\mathrm{Ir}(\mathrm{cod}) \mathrm{Cl}]_{2}(13.4 \mathrm{mg}, 20 \mu \mathrm{mol}, 5.0 \mathrm{~mol} \%),(S)-\mathbf{L} \mathbf{1}(40.6 \mathrm{mg}, 80$ $\mu \mathrm{mol}, 20 \mathrm{~mol} \%), \mathbf{2 b}(222 \mu \mathrm{L}, 1.0 \mathrm{mmol}, 2.5$ equiv), and La(OTf) 3 (17.6 $\mathrm{mg}, 30 \mu \mathrm{mol}, 7.5 \mathrm{~mol} \%$ ), and the reaction was run for 27 hours. The regiomeric ratio (rr) was determined to be $>20: 1$ by ${ }^{1} \mathrm{H}$ NMR analysis of the unpurified reaction mixture. The title compound was obtained by purification via $\mathrm{SiO}_{2}$ flash column chromatography using pentane:Et $\mathrm{E}_{2} \mathrm{O}(50: 1 \mathrm{v}: \mathrm{v})$. The title compound was obtained as a clear colorless oil $(91.5 \mathrm{mg}$, $0.27 \mathrm{mmol}, 68 \%$ yield). ${ }^{1} \mathbf{H}$ NMR $\left(400 \mathrm{MHz}, \mathrm{CDCl}_{3}\right) \delta 7.42-7.35(\mathrm{~m}, 2 \mathrm{H}), 7.27-7.18(\mathrm{~m}$, 2H), $6.06(\mathrm{t}, J=6.7 \mathrm{~Hz}, 1 \mathrm{H}), 4.82-4.65(\mathrm{~m}, 2 \mathrm{H}), 3.98(\mathrm{q}, J=7.1 \mathrm{~Hz}, 2 \mathrm{H}), 1.52(\mathrm{~s}, 3 \mathrm{H}), 1.14$ (s, 6H), 1.13 (s, 3H). ${ }^{13} \mathrm{C}$ NMR $\left(100 \mathrm{MHz}, \mathrm{CDCl}_{3}\right) \delta$ 207.6, 176.0, 143.6, 130.3 (2C), 129.9 (2C), 120.4, 96.3, 77.2, 60.4, 49.3, 46.2, 22.2, 22.1, 22.0, 13.9. IR (atr, $\left.\mathrm{cm}^{-1}\right)$ 2980, 2934, 1955, $1718,1492,1472,1396,1263,1172,1140,1085,1026,1007,839,804,733,523$. HRMS (ESI+, M+Na) Calc'd for $\mathrm{C}_{17} \mathrm{H}_{21} \mathrm{O}_{2} \mathrm{BrNa} 359.0617$, found 359.0616. Enantiomeric purity was determined by HPLC analysis in comparison with racemic material $\mathrm{T}_{\mathrm{R}}=7.1$ (minor) $\mathrm{T}_{\mathrm{R}}=7.6$ (major) (95.5:4.5 er shown); Chiracel IC column, 99.7\% hexane to $0.3 \% \mathrm{iPrOH}, 1.0 \mathrm{~mL} / \mathrm{min}$, $\left.220 \mathrm{~nm}, 483 \mathrm{psi}, 26{ }^{\circ} \mathrm{C}\right)$. $[\boldsymbol{\alpha}]_{D}^{27}=-103.3\left(\mathrm{c}=0.5, \mathrm{CHCl}_{3}\right)$. The absolute stereochemistry of this compound was assigned in analogy to $\mathbf{3 a}$.
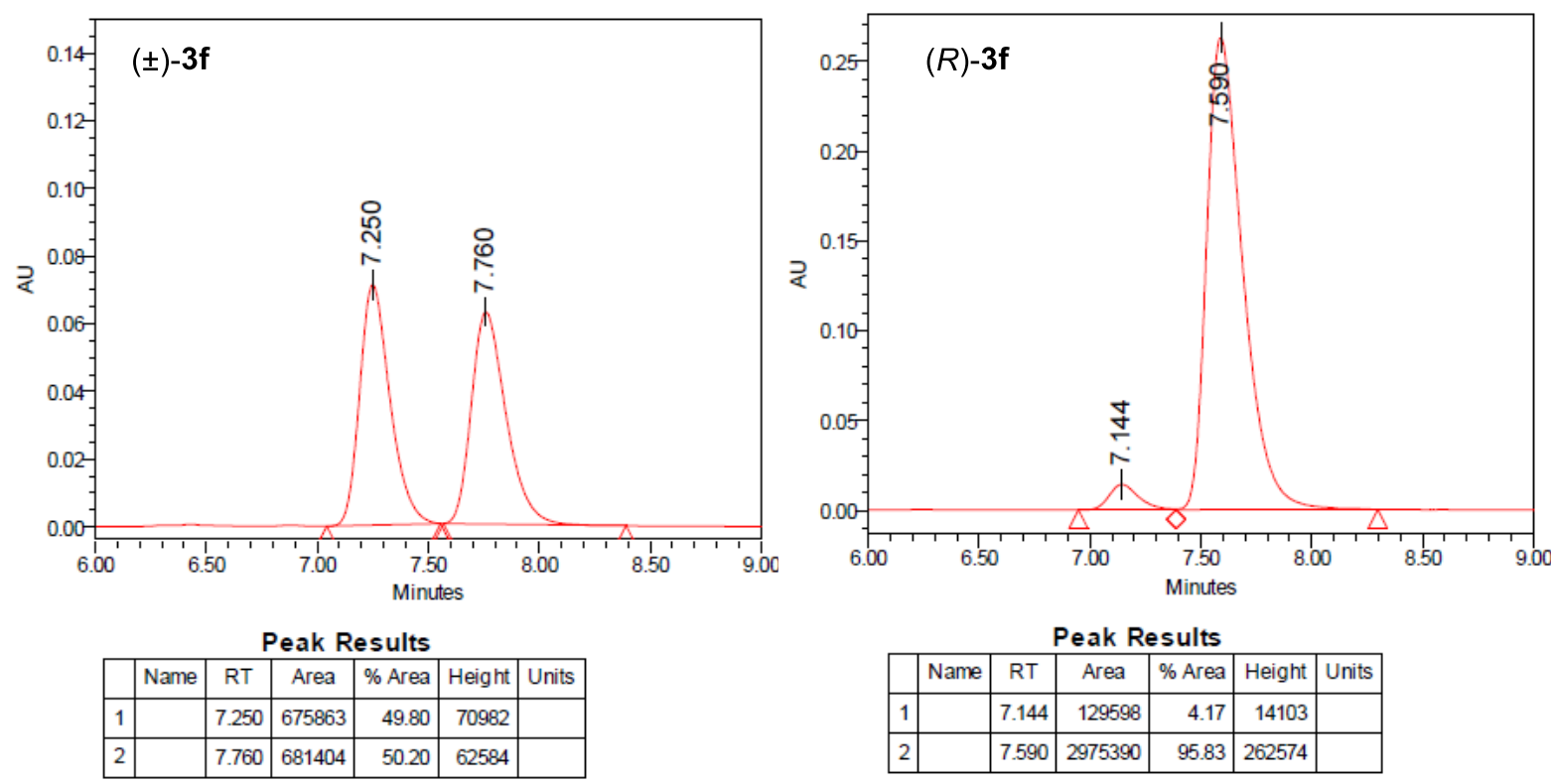


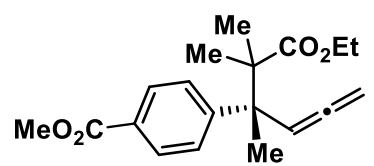

methyl (R)-4-(1-ethoxy-2,2,3-trimethyl-1-oxohexa-4,5-dien-3yl)benzoate (3g) - Was synthesized according to general procedure $2 \mathrm{~b}$ using $1 \mathrm{~g}(87.3 \mathrm{mg}, 0.4 \mathrm{mmol})$, [ $\mathrm{Ir}(\operatorname{cod}) \mathrm{Cl}]_{2}(13.4 \mathrm{mg}, 20 \mu \mathrm{mol}, 5.0$ mol\%), (S)-L1 (40.6 mg, $80 \mu \mathrm{mol}, 20 \mathrm{~mol} \%), \mathbf{2 b}(222 \mu \mathrm{L}, 1.0 \mathrm{mmol}$, 2.5 equiv), and $\mathrm{La}(\mathrm{OTf})_{3}(46.9 \mathrm{mg}, 80 \mu \mathrm{mol}, 20 \mathrm{~mol} \%)$, and the reaction was run for 20 hours. The regiomeric ratio (rr) was determined to be $>20: 1$ by ${ }^{1} \mathrm{H}$ NMR analysis of the unpurified reaction mixture. The title compound was obtained by purification via $\mathrm{SiO}_{2}$ flash column chromatography using pentane: $\mathrm{Et}_{2} \mathrm{O}(10: 1 \mathrm{v}: \mathrm{v})$. The title compound was obtained as a clear colorless oil (65.3 mg, $0.21 \mathrm{mmol}, 52 \%$ yield). ${ }^{1} \mathbf{H} \mathbf{~ N M R}\left(400 \mathrm{MHz}, \mathrm{CDCl}_{3}\right) \delta 7.96-7.89(\mathrm{~m}$, $2 \mathrm{H}), 7.46-7.39(\mathrm{~m}, 2 \mathrm{H}), 6.12(\mathrm{t}, J=6.7 \mathrm{~Hz}, 1 \mathrm{H}), 4.82-4.65(\mathrm{~m}, 2 \mathrm{H}), 3.97(\mathrm{q}, J=7.1 \mathrm{~Hz}$, 2H), $3.90(\mathrm{~s}, 3 \mathrm{H}), 1.56(\mathrm{~s}, 3 \mathrm{H}), 1.15(\mathrm{~d}, J=1.7 \mathrm{~Hz}, 6 \mathrm{H}), 1.10(\mathrm{t}, J=7.1 \mathrm{~Hz}, 3 \mathrm{H}),{ }^{13} \mathbf{C} \mathbf{~ N M R}$ $\left(100 \mathrm{MHz}, \mathrm{CDCl}_{3}\right) \delta 207.7,175.9,166.9,150.0,128.5(2 \mathrm{C}), 128.2(2 \mathrm{C}), 128.1,96.3,77.2,60.4$, 52.0, 49.4, 46.6, 22.3, 22.1, 21.9, 13.9. IR (atr, $\left.\mathrm{cm}^{-1}\right)$ 2981, 2951, 1954, 1719, 1609, 1435, 1408, 1381, 1279, 1193, 1140, 1110, 1018, 856, 773, 707, 529. HRMS (ESI+, M+Na) Calc'd for $\mathrm{C}_{19} \mathrm{H}_{24} \mathrm{O}_{4} \mathrm{Na} 339.1567$, found 339.1568. Enantiomeric purity was determined by SFC analysis in comparison with racemic material $\mathrm{T}_{\mathrm{R}}=5.0$ (major) $\mathrm{T}_{\mathrm{R}}=5.7$ (major) (98:2 er shown); Chiracel IA column, $95 \% \mathrm{CO}_{2}$ to $5 \% \mathrm{MeOH}, 2.0 \mathrm{~mL} / \mathrm{min}, 235 \mathrm{~nm}, 120 \mathrm{bar}, 25^{\circ} \mathrm{C}$ ). $[\boldsymbol{\alpha}]_{D}^{\mathbf{2 4}}=-$ $75.1\left(\mathrm{c}=0.5, \mathrm{CHCl}_{3}\right)$. The absolute stereochemistry of this compound was assigned in analogy to $3 \mathbf{a}$.
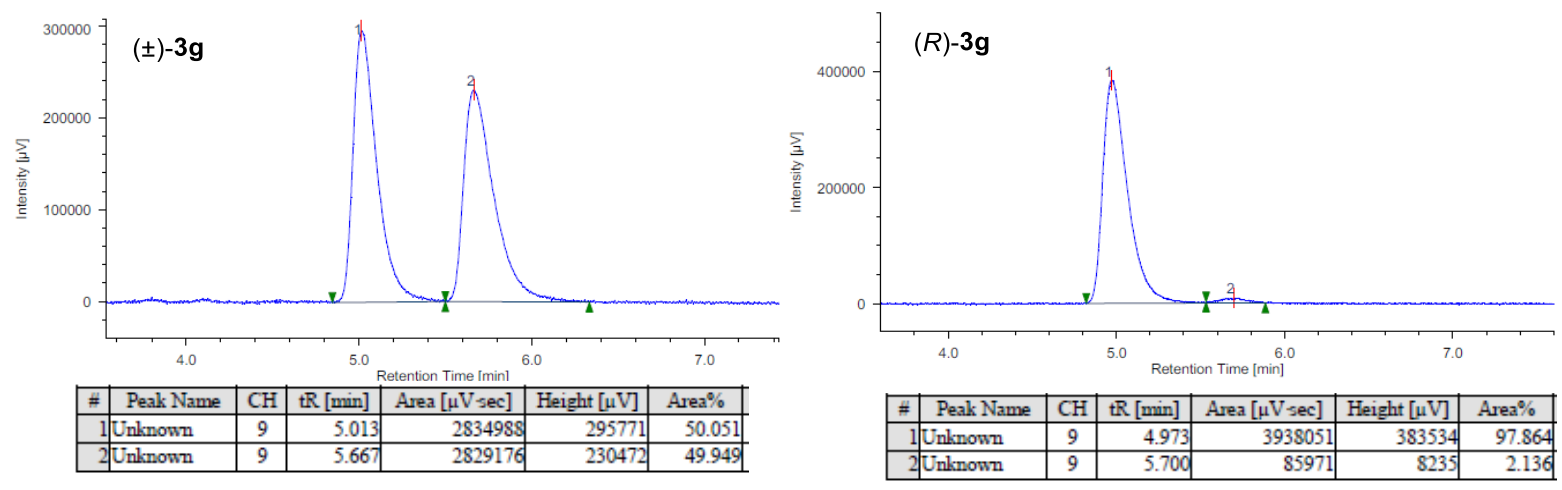


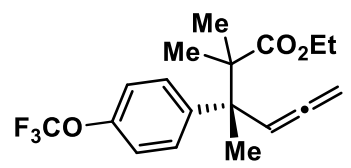

ethyl $(R)-2,2,3-$ trimethyl-3-(4-(trifluoromethoxy)phenyl)hexa-4,5-

dienoate $(\mathbf{3 h})$ - Was synthesized according to general procedure $2 \mathrm{~b}$ using $1 \mathbf{h}(97.6 \mathrm{mg}, 0.4 \mathrm{mmol}),[\operatorname{Ir}(\operatorname{cod}) \mathrm{Cl}]_{2}(13.4 \mathrm{mg}, 20 \mu \mathrm{mol}, 5.0$ mol\%), $(S)-\mathbf{L}_{1}(40.6 \mathrm{mg}, 80 \mu \mathrm{mol}, 20 \mathrm{~mol} \%), \mathbf{2 b}(222 \mu \mathrm{L}, 1.0 \mathrm{mmol}, 2.5$ equiv), and $\mathrm{La}(\mathrm{OTf})_{3}(23.4 \mathrm{mg}, 40 \mu \mathrm{mol}, 10 \mathrm{~mol} \%)$, and the reaction was run for 67 hours. The regiomeric ratio (rr) was determined to be $>20: 1$ by ${ }^{1} \mathrm{H}$ NMR analysis of the unpurified reaction mixture. The title compound was obtained by purification via $\mathrm{SiO}_{2}$ flash column chromatography using pentane: $\mathrm{Et}_{2} \mathrm{O}(50: 1 \mathrm{v}: \mathrm{v})$. The title compound was obtained as a clear colorless oil (90.9 mg, $0.27 \mathrm{mmol}, 66 \%$ yield). ${ }^{1} \mathbf{H} \mathbf{~ N M R}\left(400 \mathrm{MHz}, \mathrm{CDCl}_{3}\right) \delta 7.40-7.34(\mathrm{~m}$, $2 \mathrm{H}), 7.15-7.08(\mathrm{~m}, 2 \mathrm{H}), 6.09(\mathrm{t}, J=6.7 \mathrm{~Hz}, 1 \mathrm{H}), 4.84-4.68(\mathrm{~m}, 2 \mathrm{H}), 3.94(\mathrm{q}, J=7.1 \mathrm{~Hz}$, 2H), $1.54(\mathrm{~s}, 3 \mathrm{H}), 1.16(\mathrm{~s}, 6 \mathrm{H}), 1.08(\mathrm{t}, J=7.1 \mathrm{~Hz}, 3 \mathrm{H}) .{ }^{13} \mathrm{C}$ NMR $\left(100 \mathrm{MHz}, \mathrm{CDCl}_{3}\right) \delta$ 207.6, 176.0, $147.70-147.55(\mathrm{~m}), 143.3,129.4(2 \mathrm{C}), 120.47$ (q, $J=256.8 \mathrm{~Hz}), 119.5(2 \mathrm{C}), 96.4,77.2$, 60.4, 49.5, 46.1, 22.3, 22.0, 22.0, 13.8. ${ }^{19} \mathbf{F}$ NMR $\left(376 \mathrm{MHz}, \mathrm{CDCl}_{3}\right) \delta$-57.9. IR (atr, $\left.\mathrm{cm}^{-1}\right)$ 2982, 1955, 1718, 1510, 1473, 1381, 1257, 1214, 1164, 1086, 1018, 845, 547. HRMS (ESI+, $\mathrm{M}+\mathrm{Na}$ ) Calc'd for $\mathrm{C}_{18} \mathrm{H}_{21} \mathrm{O}_{3} \mathrm{~F}_{3} \mathrm{Na} 365.1335$, found 365.1329. Enantiomeric purity was determined by HPLC analysis in comparison with racemic material $\mathrm{T}_{\mathrm{R}}=6.6$ (minor) $\mathrm{T}_{\mathrm{R}}=7.3$ (major) (96:3 er shown); Chiracel AD-H column, 99.9\% hexane to $0.1 \% \mathrm{iPrOH}, 1.0 \mathrm{~mL} / \mathrm{min}$, $\left.214 \mathrm{~nm}, 618 \mathrm{psi}, 26{ }^{\circ} \mathrm{C}\right) .[\alpha]_{D}^{25}=-68.4\left(\mathrm{c}=0.5, \mathrm{CHCl}_{3}\right)$. The absolute stereochemistry of this compound was assigned in analogy to $\mathbf{3 a}$.
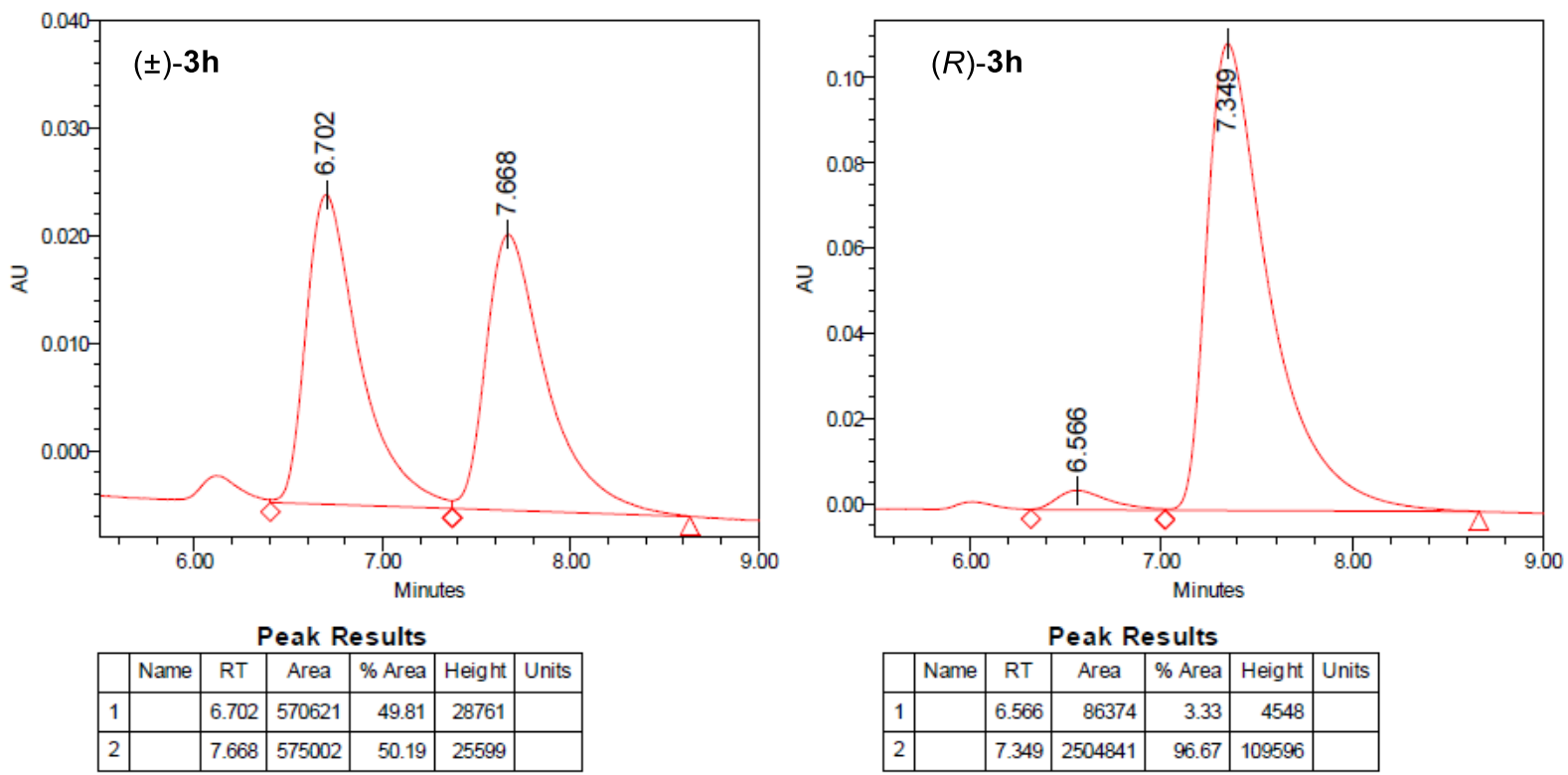


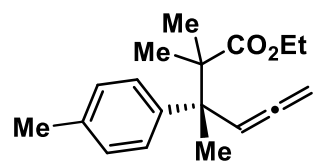

ethyl $(R)$-2,2,3-trimethyl-3-(p-tolyl)hexa-4,5-dienoate $(3 i)$ - Was synthesized according to general procedure 2a using 1i $(69.7 \mathrm{mg}, 0.4$ $\mathrm{mmol}),[\mathrm{Ir}(\mathrm{cod}) \mathrm{Cl}]_{2}(13.4 \mathrm{mg}, 20 \mu \mathrm{mol}, 5.0 \mathrm{~mol} \%),(S)-\mathbf{L} \mathbf{1}(40.6 \mathrm{mg}, 80$ $\mu \mathrm{mol}, 20 \mathrm{~mol} \%)$, $2 \mathbf{a}(424 \mu \mathrm{L}, 1.6 \mathrm{mmol}, 4.0$ equiv), and La(OTf) 3 (17.6 $\mathrm{mg}, 30 \mu \mathrm{mol}, 7.5 \mathrm{~mol} \%$ ), and the reaction was run for 23 hours. The regiomeric ratio (rr) was determined to be $>20: 1$ by ${ }^{1} \mathrm{H}$ NMR analysis of the unpurified reaction mixture. The title compound was obtained by purification via $\mathrm{SiO}_{2}$ flash column chromatography using $100 \%$ pentane to pentane:Et $\mathrm{O}_{2} \mathrm{O}(50: 1 \mathrm{v}: \mathrm{v})$. The title compound was obtained as a clear colorless oil (81.8 mg, $0.30 \mathrm{mmol}, 75 \%$ yield). ${ }^{1} \mathbf{H}$ NMR $\left(400 \mathrm{MHz}, \mathrm{CDCl}_{3}\right) \delta 7.27-7.22(\mathrm{~m}, 2 \mathrm{H}), 7.11-$ $7.05(\mathrm{~m}, 2 \mathrm{H}), 6.12(\mathrm{t}, J=6.7 \mathrm{~Hz}, 1 \mathrm{H}), 4.80-4.64(\mathrm{~m}, 2 \mathrm{H}), 3.99(\mathrm{q}, J=7.1 \mathrm{~Hz}, 2 \mathrm{H}), 2.31(\mathrm{~s}$, $3 \mathrm{H}), 1.54(\mathrm{~s}, 3 \mathrm{H}), 1.14(\mathrm{~s}, 6 \mathrm{H}), 1.13(\mathrm{t}, J=7.2 \mathrm{~Hz}, 3 \mathrm{H}) .{ }^{13} \mathbf{C} \mathbf{N M R}\left(100 \mathrm{MHz}, \mathrm{CDCl}_{3}\right) \delta 207.5$, 176.3, 141.4, 135.8, 128.0 (2C), 127.9 (2C), 96.8, 76.8, 60.2, 49.5, 45.9, 22.3, 22.1, 21.9, 20.8, 13.9. IR (atr, $\left.\mathrm{cm}^{-1}\right)$ 2979, 2946, 1954, 1716, 1514, 1462, 1379, 1262, 1171, 1137, 1085, 1020, 838, 807, 726, 529. HRMS (ESI+, M+H) Calc'd for $\mathrm{C}_{18} \mathrm{H}_{25} \mathrm{O}_{2} 273.1849$, found 273.1846. Enantiomeric purity was determined by HPLC analysis in comparison with racemic material $\mathrm{T}_{\mathrm{R}}=7.7$ (minor) $\mathrm{T}_{\mathrm{R}}=16.9$ (major) (99:1 er shown); Chiracel IC column, $99.7 \%$ hexane to $0.3 \%$ iPrOH, $\left.1.0 \mathrm{~mL} / \mathrm{min}, 217 \mathrm{~nm}, 485 \mathrm{psi}, 25{ }^{\circ} \mathrm{C}\right)[\boldsymbol{\alpha}]_{\boldsymbol{D}}^{\mathbf{2 6}}=-101.3\left(\mathrm{c}=0.5, \mathrm{CHCl}_{3}\right)$. The absolute stereochemistry of this compound was assigned in analogy to $\mathbf{3 a}$.
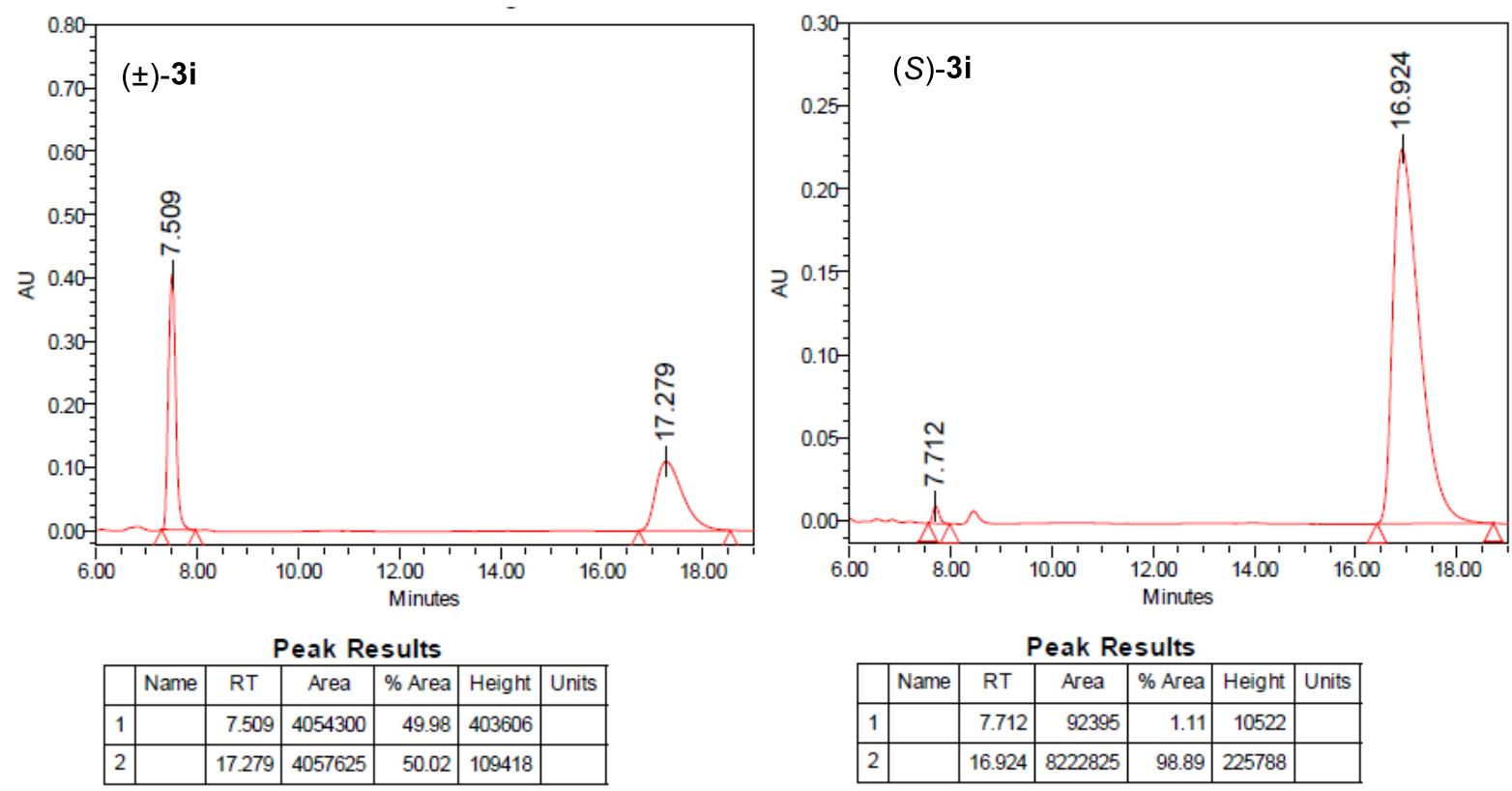


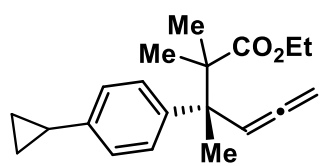

ethyl $(R)$-3-(4-cyclopropylphenyl)-2,2,3-trimethylhexa-4,5-dienoate

(3j) - Was synthesized according to general procedure $2 \mathrm{~b}$ using $\mathbf{1 j}(80.1$ $\mathrm{mg}, 0.4 \mathrm{mmol}),[\mathrm{Ir}(\mathrm{cod}) \mathrm{Cl}]_{2}(13.4 \mathrm{mg}, 20 \mu \mathrm{mol}, 5.0 \mathrm{~mol} \%),(S)-\mathbf{L} \mathbf{1}(40.6$ $\mathrm{mg}, 80 \mu \mathrm{mol}, 20 \mathrm{~mol} \%), \mathbf{2 a}\left(424 \mu \mathrm{L}, 1.6 \mathrm{mmol}, 4.0\right.$ equiv), and $\mathrm{La}(\mathrm{OTf})_{3}$ (17.6 mg, $30 \mu \mathrm{mol}, 7.5 \mathrm{~mol} \%$ ), and the reaction was run for 17 hours. The regiomeric ratio (rr) was determined to be $>20: 1$ by ${ }^{1} \mathrm{H}$ NMR analysis of the unpurified reaction mixture. The title compound was obtained by purification via $\mathrm{SiO}_{2}$ flash column chromatography using pentane: $\mathrm{Et}_{2} \mathrm{O}(50: 1 \mathrm{v}: \mathrm{v})$. The title compound was obtained as a clear colorless oil $(97.4 \mathrm{mg}$, $0.33 \mathrm{mmol}, 82 \%$ yield). ${ }^{1} \mathbf{H}$ NMR $\left(400 \mathrm{MHz} \mathrm{CDCl}_{3}\right) \delta 7.26-7.21(\mathrm{~m}, 2 \mathrm{H}), 7.01-6.94(\mathrm{~m}$, $2 \mathrm{H}), 6.11(\mathrm{t}, J=6.7 \mathrm{~Hz}, 1 \mathrm{H}), 4.82-4.63(\mathrm{~m}, 2 \mathrm{H}), 3.98(\mathrm{q}, J=7.1 \mathrm{~Hz}, 2 \mathrm{H}), 1.86(\mathrm{tt}, J=8.4,5.1$ $\mathrm{Hz}, 1 \mathrm{H}), 1.53(\mathrm{~s}, 3 \mathrm{H}), 1.18-1.07(\mathrm{~m}, 9 \mathrm{H}), 0.99-0.86(\mathrm{~m}, 2 \mathrm{H}), 0.75-0.60(\mathrm{~m}, 2 \mathrm{H}) .{ }^{13} \mathbf{C} \mathbf{~ N M R}$ $\left(100 \mathrm{MHz}, \mathrm{CDCl}_{3}\right) \delta 207.5,176.3,141.9,141.4,127.9$ (2C), 124.5 (2C), 96.7, 76.8, 60.2, 49.5, 45.9, 22.3, 22.1, 21.8, 14.9, 13.9, 9.1, 9.1. IR (atr, $\left.\mathrm{cm}^{-1}\right)$ 3083, 2979, 2934, 1954, 1718, 1517, 1461, 1380, 1264, 1172, 1139, 1086, 1017, 901, 839, 810, 558. HRMS (ESI+, M+H) Calc'd for $\mathrm{C}_{20} \mathrm{H}_{27} \mathrm{O}_{2}$ 299.2006, found 299.2011. Enantiomeric purity was determined by HPLC analysis in comparison with racemic material $\mathrm{T}_{\mathrm{R}}=5.9$ (minor) $\mathrm{T}_{\mathrm{R}}=7.6$ (major) (99:1 er shown); Chiracel IC column, $99 \%$ hexane to $1 \%$ iPrOH, $\left.1.0 \mathrm{~mL} / \mathrm{min}, 230 \mathrm{~nm}, 490 \mathrm{psi}, 26{ }^{\circ} \mathrm{C}\right)$. $[\boldsymbol{\alpha}]_{\boldsymbol{D}}^{\mathbf{2 5}}=$ $-98.1\left(\mathrm{c}=0.5, \mathrm{CHCl}_{3}\right)$. The absolute stereochemistry of this compound was assigned in analogy to $3 \mathbf{a}$.
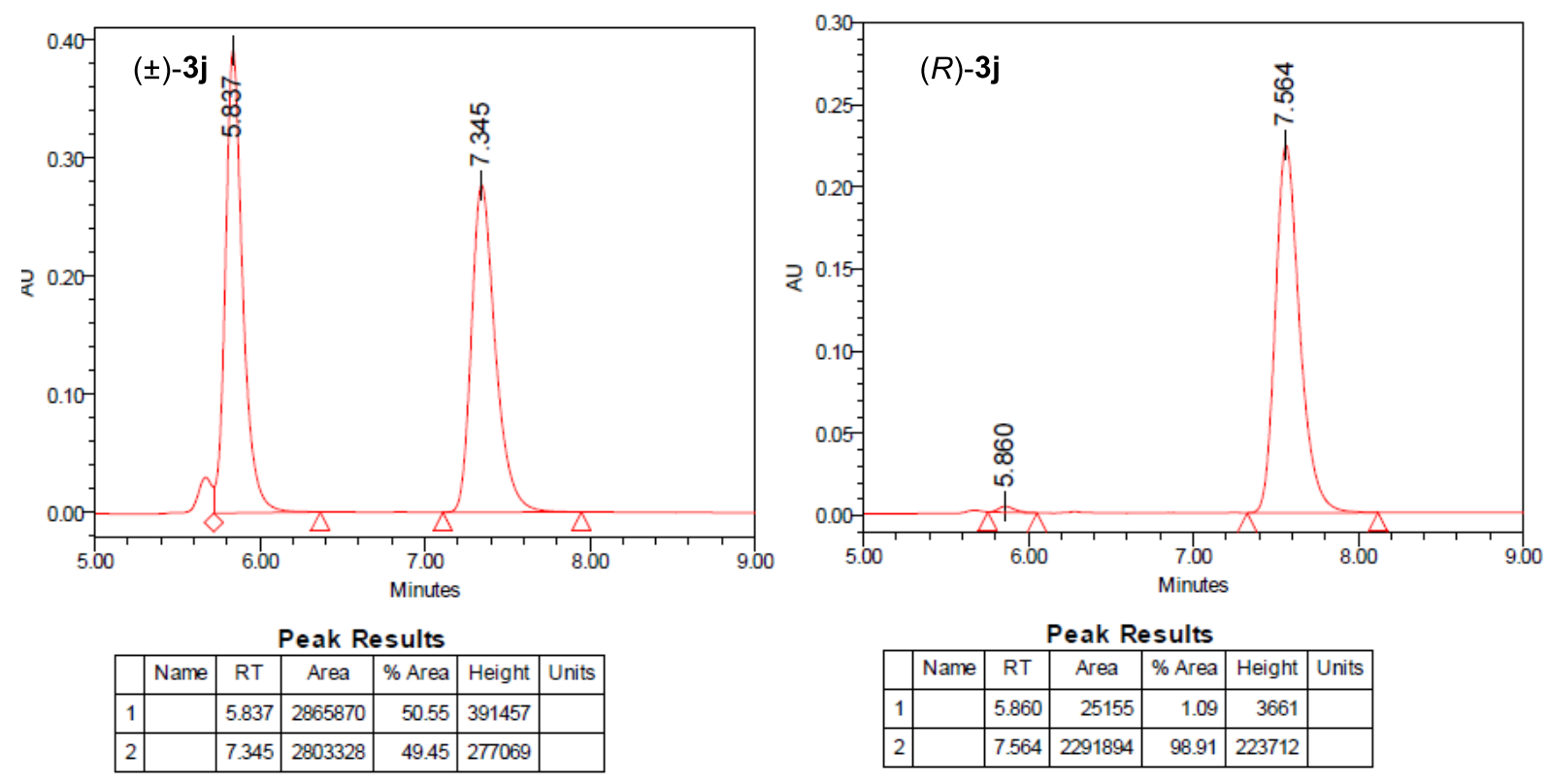


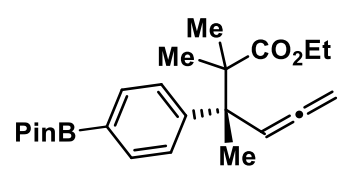

ethyl

(R)-2,2,3-trimethyl-3-(4-(4,4,5,5-tetramethyl-1,3,2-

dioxaborolan-2-yl)phenyl)hexa-4,5-dienoate (3k) - Was synthesized according to general procedure $2 \mathrm{a}$ using $1 \mathbf{k}(114.5 \mathrm{mg}, 0.4 \mathrm{mmol})$, $\left[\operatorname{Ir}(\operatorname{cod}) \mathrm{Cl}_{2}(13.4 \mathrm{mg}, 20 \mu \mathrm{mol}, 5.0 \mathrm{~mol} \%),(S)-\mathbf{L}_{1}(40.6 \mathrm{mg}, 80 \mu \mathrm{mol}\right.$, $20 \mathrm{~mol} \%), \mathbf{2 b}(222 \mu \mathrm{L}, 1.0 \mathrm{mmol}, 2.5$ equiv), and La(OTf) $3(17.6 \mathrm{mg}, 30 \mu \mathrm{mol}, 7.5 \mathrm{~mol} \%)$, and the reaction was run for 24 hours. The regiomeric ratio (rr) was determined to be $>20: 1$ by ${ }^{1} \mathrm{H}$ NMR analysis of the unpurified reaction mixture. The title compound was obtained by purification via $\mathrm{SiO}_{2}$ flash column chromatography using pentane:Et ${ }_{2} \mathrm{O}(8: 1 \mathrm{v}: \mathrm{v})$. The title compound was obtained as a clear colorless oil (125.4 mg, $0.33 \mathrm{mmol}, 82 \%$ yield). ${ }^{1} \mathbf{H}$ NMR $\left(400 \mathrm{MHz}, \mathrm{CDCl}_{3}\right) \delta 7.75-7.67(\mathrm{~m}, 2 \mathrm{H}), 7.40-7.31(\mathrm{~m}, 2 \mathrm{H}), 6.13(\mathrm{t}, J=6.7 \mathrm{~Hz}, 1 \mathrm{H}), 4.80-$ $4.62(\mathrm{~m}, 2 \mathrm{H}), 3.99$ (q, $J=7.1 \mathrm{~Hz}, 2 \mathrm{H}), 1.55(\mathrm{~s}, 3 \mathrm{H}), 1.33(\mathrm{~s}, 12 \mathrm{H}), 1.16-1.10(\mathrm{~m}, 9 \mathrm{H}) .{ }^{13} \mathrm{C}$ NMR $\left(100 \mathrm{MHz}, \mathrm{CDCl}_{3}\right) \delta$ 207.6, 176.2, 147.8, 133.8 (2C), 127.5 (2C), 96.6, 83.7 (2C), 76.9, $60.3,49.4,46.5,24.8$ (4C), 22.3, 22.2, 21.9, 13.9. (NOTE: one aromatic carbon peak could not be observed due to quadrupole relaxation) ${ }^{11} \mathbf{B} \mathbf{N M R}\left(128 \mathrm{MHz}, \mathrm{CDCl}_{3}\right) \delta-52.3$. IR (atr, $\left.\mathrm{cm}^{-1}\right)$ 2978, 2937, 1955, 1717, 1609, 1469, 1400, 1359, 1324, 1262, 1141, 1096, 1018, 962, 859, 842, 754, 660, 578, 453. HRMS (ESI+, M+H) Calc'd for $\mathrm{C}_{23} \mathrm{H}_{34} \mathrm{BO}_{4}$ 385.2549, found 385.2549. Enantiomeric purity was determined by HPLC analysis in comparison with racemic material $\mathrm{T}_{\mathrm{R}}=5.2$ (minor) $\mathrm{T}_{\mathrm{R}}=5.8$ (major) (96.5:3.5 er shown); Chiracel IC column, $99 \%$ hexane to $1 \%$ iPrOH, $\left.1.0 \mathrm{~mL} / \mathrm{min}, 227 \mathrm{~nm}, 495 \mathrm{psi}, 26{ }^{\circ} \mathrm{C}\right) .[\boldsymbol{\alpha}]_{\boldsymbol{D}}^{25}=-84.2\left(\mathrm{c}=0.5, \mathrm{CHCl}_{3}\right)$. The absolute stereochemistry of this compound was assigned in analogy to $\mathbf{3 a}$.
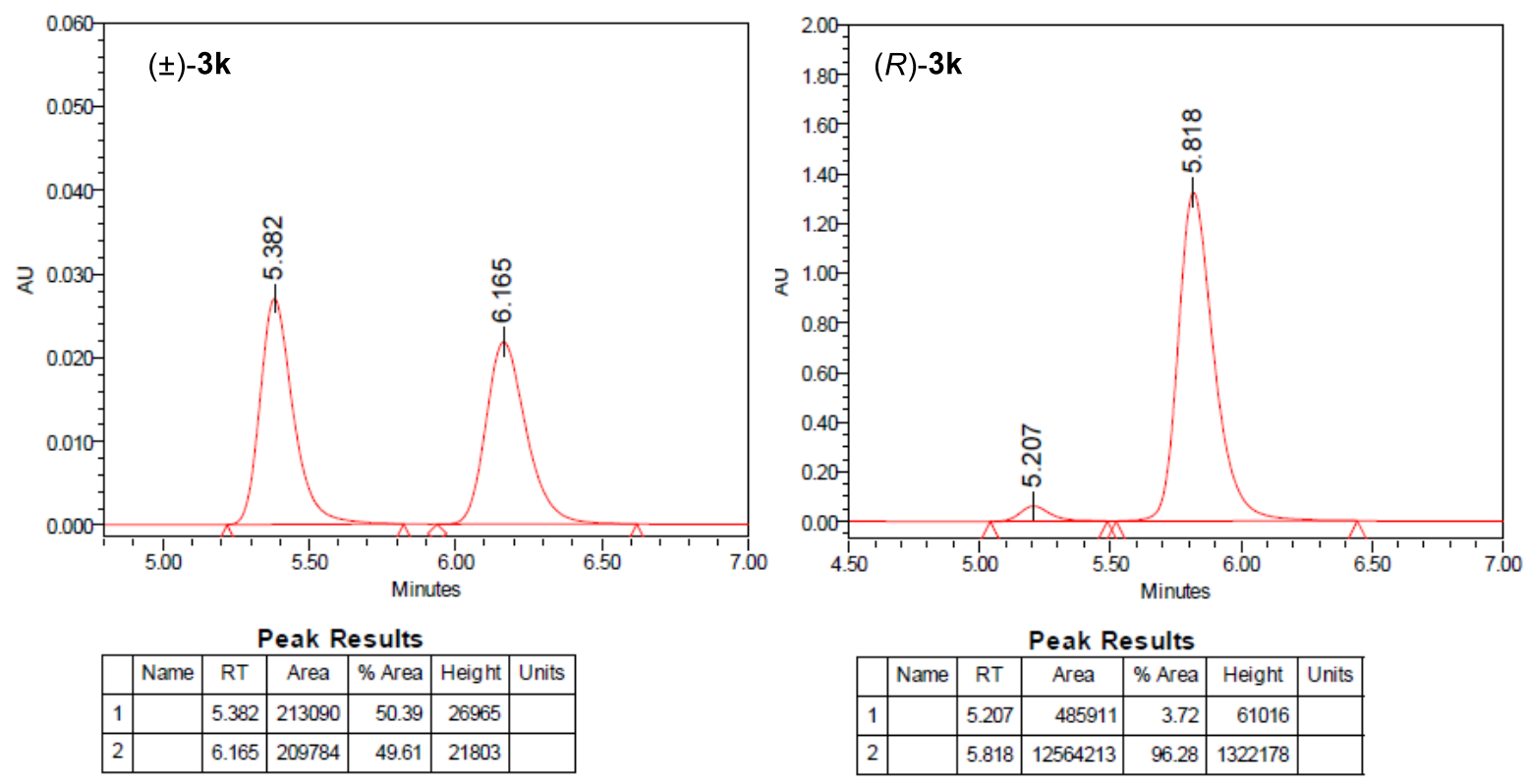


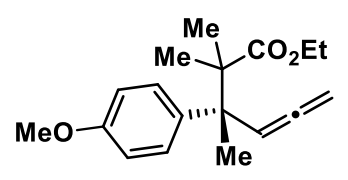

ethyl (R)-3-(4-methoxyphenyl)-2,2,3-trimethylhexa-4,5-dienoate (31)

- Was synthesized according to general procedure $2 \mathrm{~b}$ using $11(76.1 \mathrm{mg}$, $0.4 \mathrm{mmol}),[\mathrm{Ir}(\mathrm{cod}) \mathrm{Cl}]_{2}(13.4 \mathrm{mg}, 20 \mu \mathrm{mol}, 5.0 \mathrm{~mol} \%),(S)-\mathbf{L} \mathbf{1}(40.6 \mathrm{mg}$, $80 \mu \mathrm{mol}, 20 \mathrm{~mol} \%), 2 \mathbf{a}\left(265 \mu \mathrm{L}, 1.0 \mathrm{mmol}, 2.5\right.$ equiv), and $\mathrm{La}(\mathrm{OTf})_{3}(7.0$ (rr) was determined to be 10:1 by ${ }^{1} \mathrm{H}$ NMR analysis of the unpurified reaction mixture. The title compound was obtained as a mixture with inseparable 1,3-diene isomer by purification via $\mathrm{SiO}_{2}$ flash column chromatography using pentane: $\mathrm{Et}_{2} \mathrm{O}(30: 1$ to $20: 1 \mathrm{v}: \mathrm{v})$. The title compound was obtained as a clear colorless oil $\left(87.7 \mathrm{mg}, 0.30 \mathrm{mmol}, 76 \%\right.$ yield). ${ }^{\mathbf{1}} \mathbf{H} \mathbf{~ N M R}\left(400 \mathrm{MHz}, \mathrm{CDCl}_{3}\right)$ $\delta 7.30-7.24(\mathrm{~m}, 2 \mathrm{H}), 6.83-6.77(\mathrm{~m}, 2 \mathrm{H}), 6.10(\mathrm{t}, J=6.7 \mathrm{~Hz}, 1 \mathrm{H}), 4.79-4.66(\mathrm{~m}, 2 \mathrm{H}), 3.99$ $(\mathrm{q}, J=7.1 \mathrm{~Hz}, 2 \mathrm{H}), 3.79(\mathrm{~s}, 3 \mathrm{H}), 1.56-1.47(\mathrm{~m}, 3 \mathrm{H}), 1.17-1.09(\mathrm{~m}, 9 \mathrm{H}) .{ }^{13} \mathbf{C}$ NMR $(100$ $\left.\mathrm{MHz}, \mathrm{CDCl}_{3}\right) \delta 207.5,176.3,157.8,136.5,129.0,112.5,96.8,76.8,60.2,55.1,49.5,45.6$, i22.3, 22.1, 22.0, 13.9. IR (atr, cm ${ }^{-1}$ ) 2979, 2938, 2836, 1954, 1717, 1608, 1513, 1464, 1444, 1379, 1295, 1252, 1188, 1138, 1085, 1033, 839, 816, 544. HRMS (ESI+, M+H) Calc'd for $\mathrm{C}_{18} \mathrm{H}_{25} \mathrm{O}_{3} 289.1798$, found 289.1798. Enantiomeric purity was determined by HPLC analysis in comparison with racemic material $\mathrm{T}_{\mathrm{R}}=10.1$ (minor) $\mathrm{T}_{\mathrm{R}}=11.7$ (major) (98:2 er shown); Chiracel IC column, 99\% hexane to $\left.1 \% \mathrm{iPrOH}, 1.0 \mathrm{~mL} / \mathrm{min}, 222 \mathrm{~nm}, 490 \mathrm{psi}, 26{ }^{\circ} \mathrm{C}\right)[\boldsymbol{\alpha}]_{\boldsymbol{D}}^{\mathbf{2 4}}=-$ $93.4\left(\mathrm{c}=0.5, \mathrm{CHCl}_{3}\right)$. The absolute stereochemistry of this compound was assigned in analogy to $3 \mathbf{a}$.
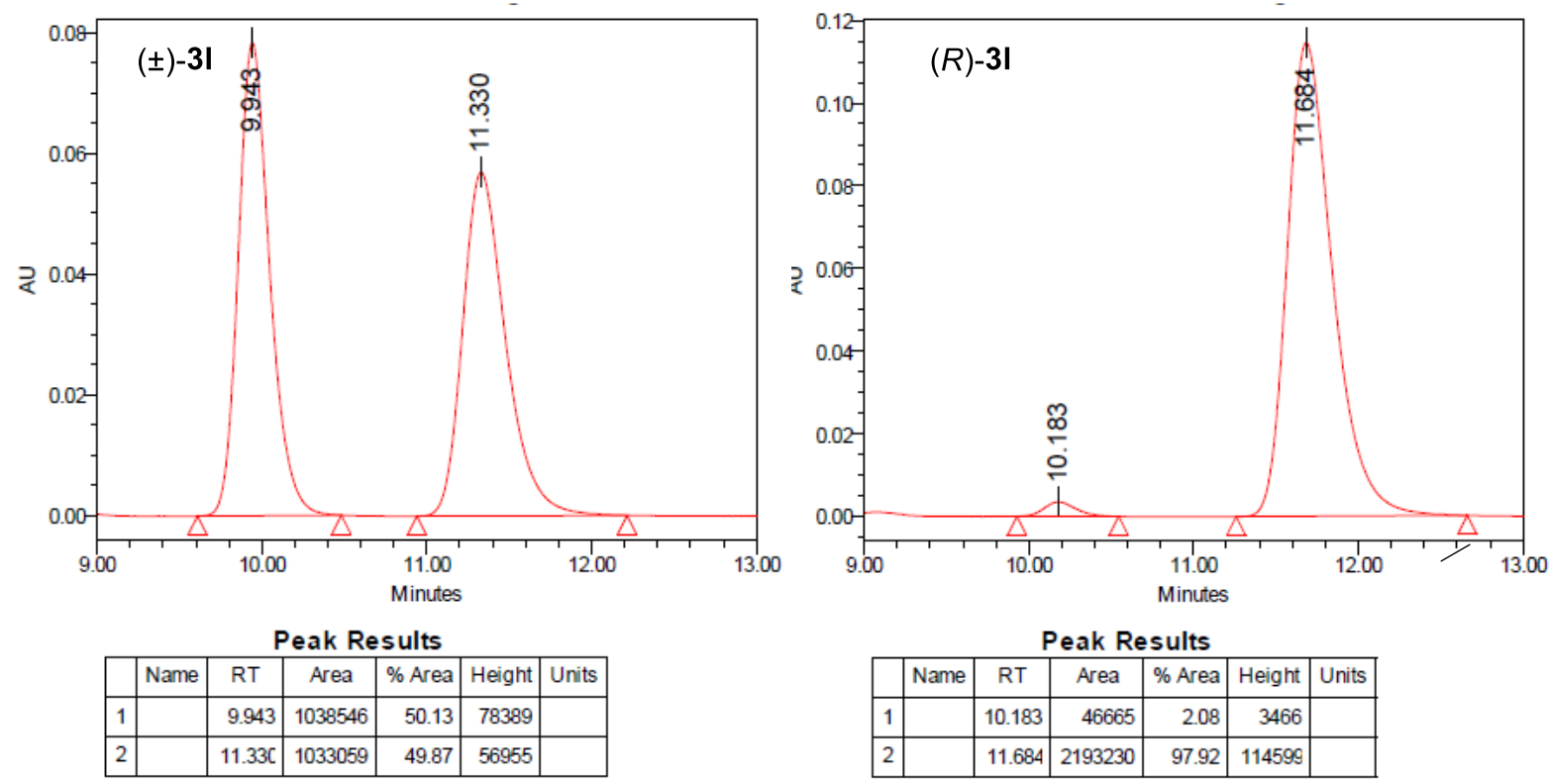

Characterization data for 1, 3-diene byproduct 31':

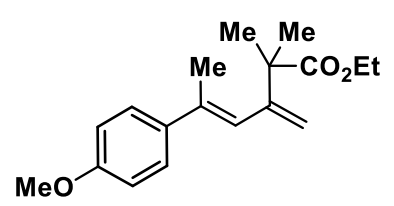

${ }^{1} \mathbf{H}$ NMR $\left(400 \mathrm{MHz}, \mathrm{CDCl}_{3}\right) \delta 7.38-7.32(\mathrm{~m}, 2 \mathrm{H}), 6.89-6.85(\mathrm{~m}$, $2 \mathrm{H}), 6.16(\mathrm{t}, J=1.4 \mathrm{~Hz}, 1 \mathrm{H}), 5.28(\mathrm{~d}, J=1.2 \mathrm{~Hz}, 1 \mathrm{H}), 5.03-4.98(\mathrm{~m}$, $1 \mathrm{H}), 4.11(\mathrm{q}, J=7.1 \mathrm{~Hz}, 2 \mathrm{H}), 3.81(\mathrm{~s}, 3 \mathrm{H}), 2.12(\mathrm{~d}, J=1.5 \mathrm{~Hz}, 3 \mathrm{H})$, $1.38(\mathrm{~s}, 6 \mathrm{H}), 1.22(\mathrm{~d}, J=7.1 \mathrm{~Hz}, 3 \mathrm{H}) .{ }^{13} \mathbf{C ~ N M R}\left(100 \mathrm{MHz}, \mathrm{CDCl}_{3}\right) \delta$ $176.3,158.8,149.0,137.5,136.0,126.9,124.8,113.5,112.6,112.5$, $60.6,55.2,47.7,24.7,17.3,14.1$. (NOTE: carbonyl carbon peak was found to overlap with the major allene product during HMBC measurements on the mixture of compounds.) 
<smiles>C=C[C@](C)(c1cccc(OC)c1)C(C)(C)C(=O)OCC</smiles>

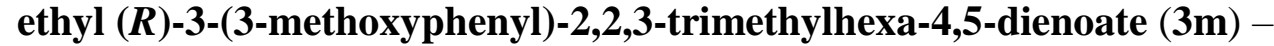
Was synthesized according to general procedure $2 \mathrm{~b}$ using $1 \mathbf{m}(76.1 \mathrm{mg}, 0.4$ $\mathrm{mmol}),\left[\mathrm{Ir}(\mathrm{cod}) \mathrm{Cl}_{2}(13.4 \mathrm{mg}, 20 \mu \mathrm{mol}, 5.0 \mathrm{~mol} \%),(S)-\mathbf{L} \mathbf{1}(40.6 \mathrm{mg}, 80\right.$ $\mu \mathrm{mol}, 20 \mathrm{~mol} \%), \mathbf{2 b}(222 \mu \mathrm{L}, 1.0 \mathrm{mmol}, 2.5 \text { equiv), and La(OTf })_{3}(17.6 \mathrm{mg}$, $30 \mu \mathrm{mol}, 7.5 \mathrm{~mol} \%$ ), and the reaction was run for 8 hours. The regiomeric ratio (rr) was determined to be $>20: 1$ by ${ }^{1} \mathrm{H}$ NMR analysis of the unpurified reaction mixture. The title compound was obtained by purification via $\mathrm{SiO}_{2}$ flash column chromatography using pentane:Et ${ }_{2} \mathrm{O}(20: 1 \mathrm{v}: \mathrm{v})$. The title compound was obtained as a clear colorless oil $(82.1 \mathrm{mg}$, $0.29 \mathrm{mmol}, 71 \%$ yield $).{ }^{1} \mathbf{H}$ NMR $\left(400 \mathrm{MHz}, \mathrm{CDCl}_{3}\right) \delta 7.19(\mathrm{t}, J=8.0 \mathrm{~Hz}, 1 \mathrm{H}), 6.97-6.91(\mathrm{~m}$, $2 \mathrm{H}), 6.78-6.73(\mathrm{~m}, 1 \mathrm{H}), 6.10(\mathrm{t}, J=6.7 \mathrm{~Hz}, 1 \mathrm{H}), 4.80-4.67(\mathrm{~m}, 2 \mathrm{H}), 4.00(\mathrm{t}, J=7.2 \mathrm{~Hz}, 2 \mathrm{H})$, $3.80(\mathrm{~s}, 3 \mathrm{H}), 1.54(\mathrm{~s}, 3 \mathrm{H}), 1.16(\mathrm{~s}, 6 \mathrm{H}), 1.12(\mathrm{t}, J=7.1 \mathrm{~Hz}, 3 \mathrm{H}),{ }^{13} \mathbf{C} \mathbf{~ N M R}\left(100 \mathrm{MHz}, \mathrm{CDCl}_{3}\right)$ $\delta 207.6,176.2,158.7,146.2,128.1,120.6,115.0,110.8,96.6,76.9,60.3,55.2,49.5,46.3,22.4$, 22.2, 21.9, 13.9. IR (atr, $\mathrm{cm}^{-1}$ ) 2980, 2940, 2834, 1955, 1717, 1599, 1581, 1490, 1464, 1429, 1380, 1263, 1172, 1138, 1084, 1046, 842, 778, 713, 699. HRMS (ESI+, M+Na) Calc'd for $\mathrm{C}_{18} \mathrm{H}_{24} \mathrm{O}_{3} \mathrm{Na} 311.1618$, found 311.1617. Enantiomeric purity was determined by HPLC analysis in comparison with racemic material $\mathrm{T}_{\mathrm{R}}=8.5$ (minor) $\mathrm{T}_{\mathrm{R}}=12.7$ (major) (97:3 er shown); Chiracel IC column, $99 \%$ hexane to $\left.1 \% \mathrm{iPrOH}, 1.0 \mathrm{~mL} / \mathrm{min}, 210 \mathrm{~nm}, 490 \mathrm{psi}, 26^{\circ} \mathrm{C}\right)[\boldsymbol{\alpha}]_{\boldsymbol{D}}^{\mathbf{2 5}}=-$ $82.6\left(\mathrm{c}=0.5, \mathrm{CHCl}_{3}\right)$. The absolute stereochemistry of this compound was assigned in analogy to $3 \mathbf{a}$.
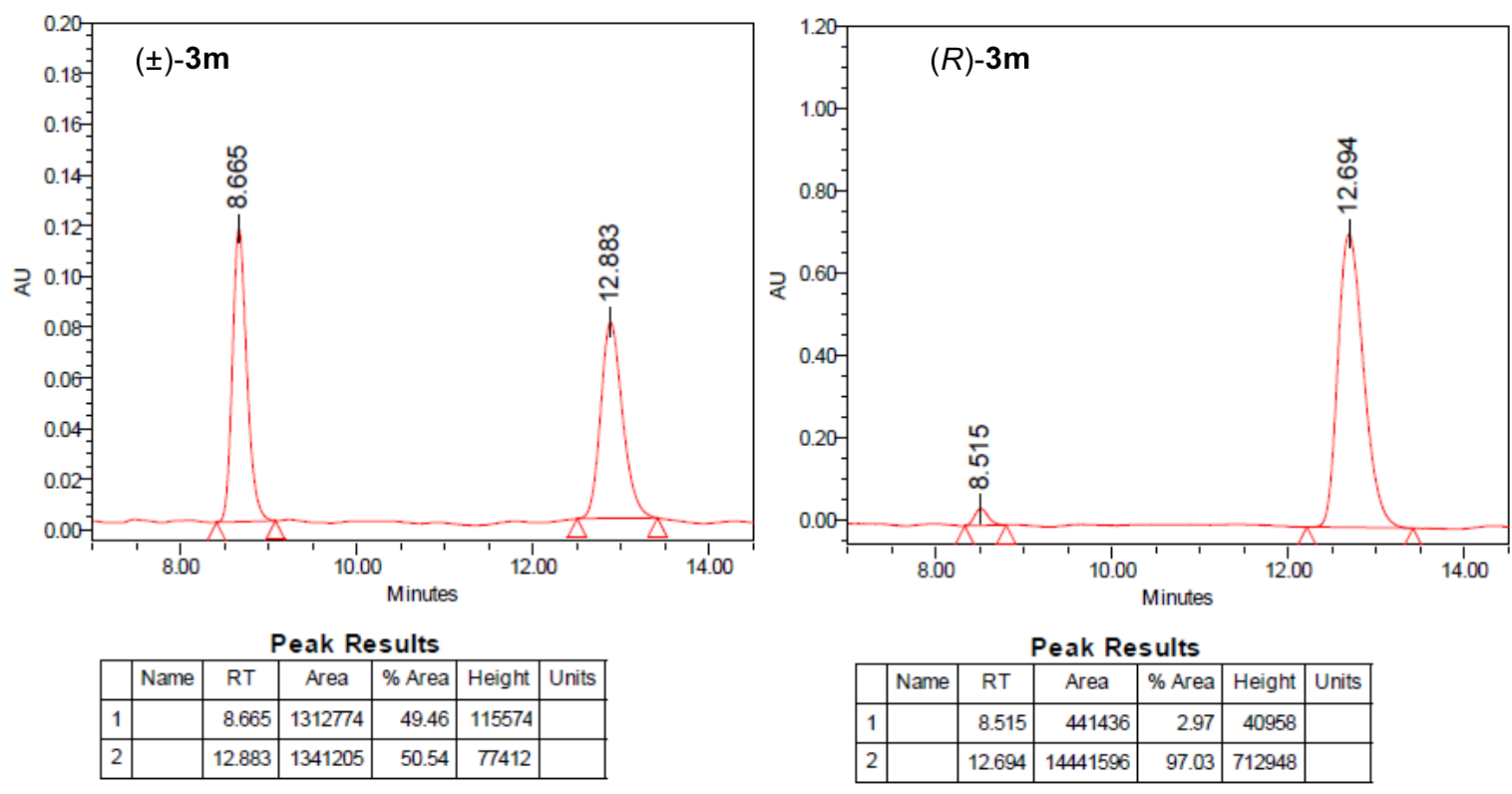


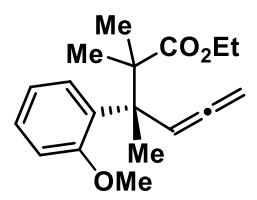

ethyl $(R)$-3-(2-methoxyphenyl)-2,2,3-trimethylhexa-4,5-dienoate (3n) Was synthesized according to general procedure $2 \mathrm{~b}$ using $1 \mathrm{n}(76.1 \mathrm{mg}, 0.4$ $\mathrm{mmol}),[\mathrm{Ir}(\mathrm{cod}) \mathrm{Cl}]_{2}(13.4 \mathrm{mg}, 20 \mu \mathrm{mol}, 5.0 \mathrm{~mol} \%),(S)-\mathbf{L} 1(40.6 \mathrm{mg}, 80 \mu \mathrm{mol}$, $20 \mathrm{~mol} \%), 2 \mathbf{b}\left(222 \mu \mathrm{L}, 1.0 \mathrm{mmol}, 2.5\right.$ equiv), and $\mathrm{La}(\mathrm{OTf})_{3}(17.6 \mathrm{mg}, 30 \mu \mathrm{mol}$, $7.5 \mathrm{~mol} \%$ ), and the reaction was run for 3 hours. The regiomeric ratio (rr) was determined to be $>20: 1$ by ${ }^{1} \mathrm{H}$ NMR analysis of the unpurified reaction mixture. The title compound was obtained by purification via $\mathrm{SiO}_{2}$ flash column chromatography using pentane: $\mathrm{Et}_{2} \mathrm{O}(20: 1 \mathrm{v}: \mathrm{v})$. The title compound was obtained as a clear colorless oil $(59.5 \mathrm{mg}$, $0.21 \mathrm{mmol}, 52 \%$ yield). ${ }^{1} \mathbf{H}$ NMR $\left(400 \mathrm{MHz}, \mathrm{CDCl}_{3}\right) \delta 7.30(\mathrm{dd}, J=7.9,1.7 \mathrm{~Hz}, 1 \mathrm{H}), 7.23-$ $7.16(\mathrm{~m}, 1 \mathrm{H}), 6.92-6.82(\mathrm{~m}, 2 \mathrm{H}), 6.48(\mathrm{t}, J=6.7 \mathrm{~Hz}, 1 \mathrm{H}), 4.79-4.58(\mathrm{~m}, 2 \mathrm{H}), 4.01(\mathrm{q}, J=$ $7.2 \mathrm{~Hz}, 2 \mathrm{H}), 3.75$ (s, 3H), 1.61 (s, 3H), $1.19-1.12(\mathrm{~m}, 9 \mathrm{H}) .{ }^{13} \mathbf{C} \mathbf{N M R}\left(100 \mathrm{MHz}, \mathrm{CDCl}_{3}\right) \delta$ 207.5, 176.4, 158.4, 133.0, 130.4, 127.9, 119.9, 112.4, 97.8, 76.4, 60.1, 55.3, 49.5, 46.6, 22.9, 22.8, 22.6, 14.0. IR (atr, $\mathrm{cm}^{-1}$ ) 3063, 2978, 2933, 1954, 1721, 1596, 1579, 1491, 1464, 1434, $1380,1289,1246,1177,1086,1028,885,840,752$. HRMS (ESI+, M+H) Calc'd for $\mathrm{C}_{18} \mathrm{H}_{25} \mathrm{O}_{3}$ 289.1798, found 289.1797. Enantiomeric purity was determined by HPLC analysis in comparison with racemic material $\mathrm{T}_{\mathrm{R}}=8.1$ (minor) $\mathrm{T}_{\mathrm{R}}=13.0$ (major) (95:5 er shown); Chiracel IC column, $99 \%$ hexane to $\left.1 \% \mathrm{iPrOH}, 1.0 \mathrm{~mL} / \mathrm{min}, 213 \mathrm{~nm}, 488 \mathrm{psi}, 26{ }^{\circ} \mathrm{C}\right)[\boldsymbol{\alpha}]_{\boldsymbol{D}}^{\mathbf{2 5}}=-55.2(\mathrm{c}=$ $\left.0.5, \mathrm{CHCl}_{3}\right)$. The absolute stereochemistry of this compound was assigned in analogy to $\mathbf{3 a}$.
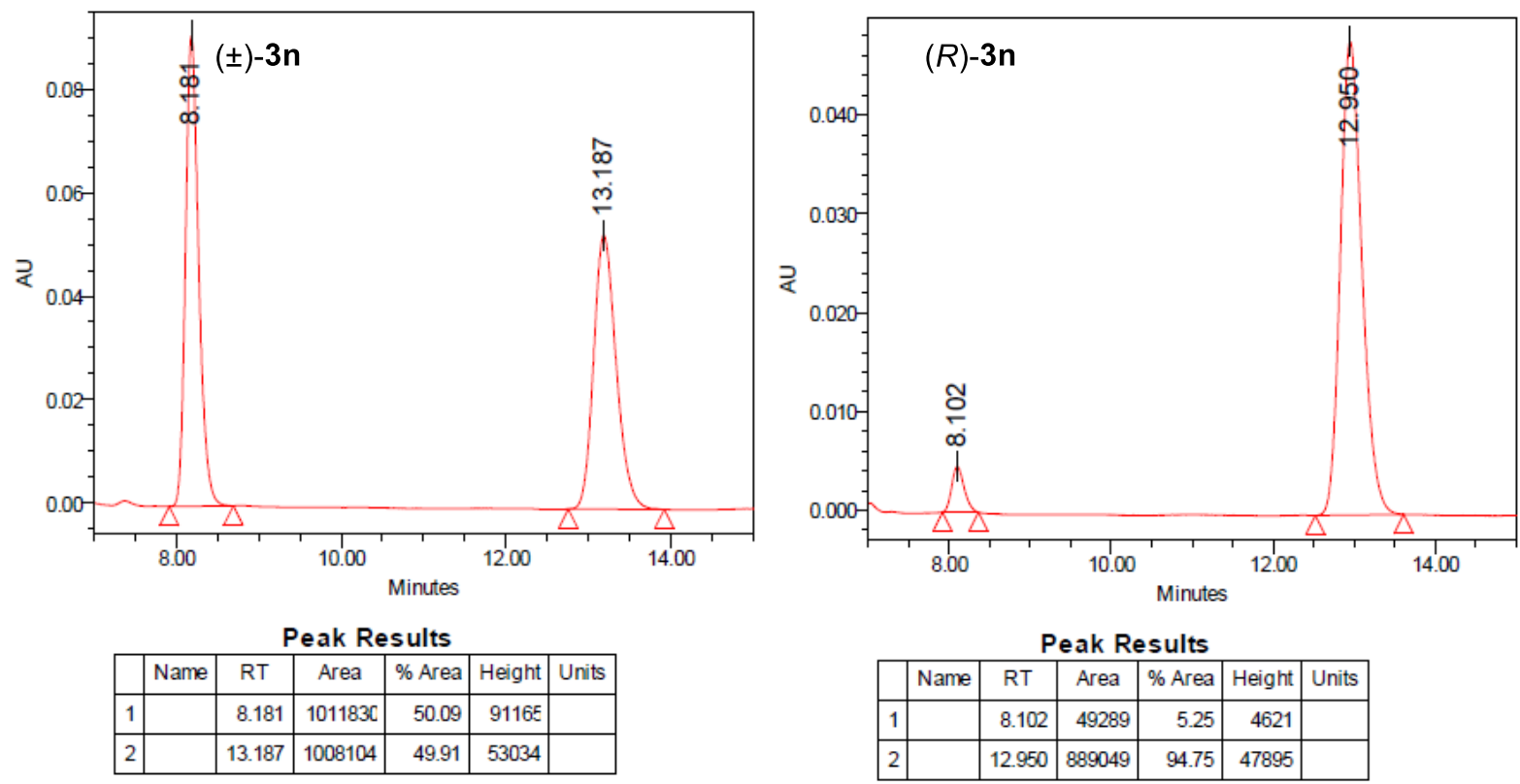
<smiles>C=C[C@](C)(c1cn([As])c2ccccc12)C(C)(C)C(=O)OCC</smiles>

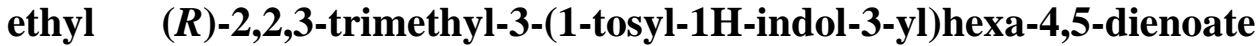

(3o) - Was synthesized according to general procedure $2 \mathrm{~b}$ using 10 (141.4 $\mathrm{mg}, 0.4 \mathrm{mmol}),[\operatorname{Ir}(\mathrm{cod}) \mathrm{Cl}]_{2}(13.4 \mathrm{mg}, 20 \mu \mathrm{mol}, 5.0 \mathrm{~mol} \%),(S)-\mathbf{L}_{1}(40.6$ $\mathrm{mg}, 80 \mu \mathrm{mol}, 20 \mathrm{~mol} \%)$, $2 \mathbf{a}\left(265 \mu \mathrm{L}, 1.0 \mathrm{mmol}, 2.5\right.$ equiv), and $\mathrm{La}(\mathrm{OTf})_{3}$ (17.6 $\mathrm{mg}, 30 \mu \mathrm{mol}, 7.5 \mathrm{~mol} \%$ ), and the reaction was run for 27 hours. The regiomeric ratio (rr) was determined to be $>20: 1$ by ${ }^{1} \mathrm{H}$ NMR analysis of the unpurified reaction mixture. The title compound was obtained by purification via $\mathrm{SiO}_{2}$ flash column chromatography using pentane: $\mathrm{Et}_{2} \mathrm{O}(10: 1 \mathrm{v}: \mathrm{v})$. The title compound was obtained as a clear colorless oil (117.3 mg, $0.26 \mathrm{mmol}, 65 \%$ yield). ${ }^{1} \mathbf{H}$ NMR $\left(400 \mathrm{MHz}, \mathrm{CDCl}_{3}\right) \delta 7.98-7.92(\mathrm{~m}$, $1 \mathrm{H}), 7.75-7.65(\mathrm{~m}, 3 \mathrm{H}), 7.43(\mathrm{~s}, 1 \mathrm{H}), 7.26-7.11(\mathrm{~m}, 4 \mathrm{H}), 6.15(\mathrm{t}, J=6.7 \mathrm{~Hz}, 1 \mathrm{H}), 4.78-$ $4.58(\mathrm{~m}, 2 \mathrm{H}), 3.80(\mathrm{qd}, J=7.2,4.6 \mathrm{~Hz}, 2 \mathrm{H}), 2.33(\mathrm{~s}, 3 \mathrm{H}), 1.62(\mathrm{~s}, 3 \mathrm{H}), 1.21(\mathrm{~d}, J=9.8 \mathrm{~Hz}, 6 \mathrm{H})$, $0.84(\mathrm{t}, J=7.1 \mathrm{~Hz}, 3 \mathrm{H}) .{ }^{13} \mathbf{C} \mathbf{~ N M R}\left(100 \mathrm{MHz}, \mathrm{CDCl}_{3}\right) \delta 208.0,176.2,144.8,135.2,135.1$, 130.6, 129.7 (2C), 126.8, 126.8 (2C), 125.1, 123.9, 123.2, 122.6, 113.5, 96.1, 77.5, 60.5, 49.3, 44.4, 23.0, 22.6, 22.6, 21.5, 13.5. IR (atr, $\left.\mathrm{cm}^{-1}\right)$ 2981, 2938, 1954, 1716, 1597, 1446, 1370, 1266, 1174, 1128, 1089, 1028, 963, 845, 812, 746, 720, 682, 665, 583, 573, 538. HRMS (ESI+, $\mathrm{M}+\mathrm{Na}$ ) Calc'd for $\mathrm{C}_{26} \mathrm{H}_{29} \mathrm{NO}_{4} \mathrm{SNa}$ 474.1710, found 474.1707. Enantiomeric purity was determined by SFC analysis in comparison with racemic material $\mathrm{T}_{\mathrm{R}}=11.0$ (major) $\mathrm{T}_{\mathrm{R}}=12.2$ (minor) (96.5:3.5 er shown); Chiracel IC column, 90\% $\mathrm{CO}_{2}$ to $10 \% \mathrm{MeOH}, 2.0 \mathrm{~mL} / \mathrm{min}, 210$ $\mathrm{nm}, 125$ bar, $\left.25{ }^{\circ} \mathrm{C}\right)[\boldsymbol{\alpha}]_{\boldsymbol{D}}^{28}=-92.0\left(\mathrm{c}=0.5, \mathrm{CHCl}_{3}\right)$. The absolute stereochemistry of this compound was assigned in analogy to $\mathbf{3 a}$.
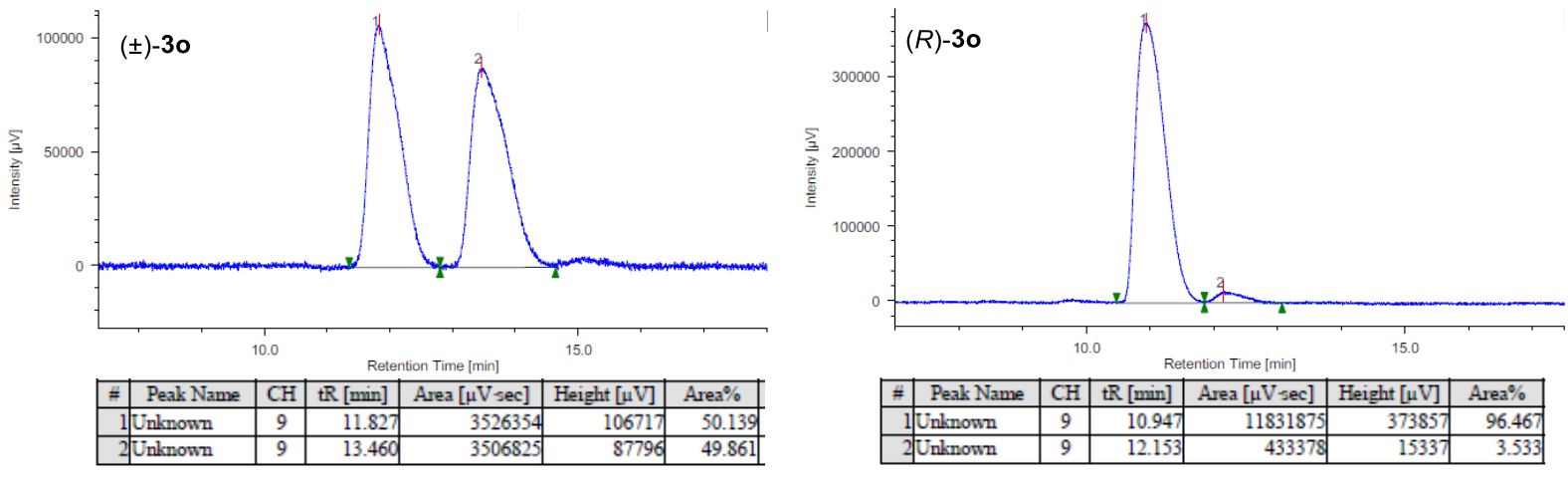


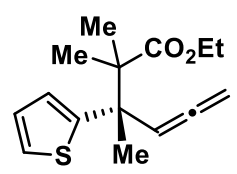

ethyl (S)-2,2,3-trimethyl-3-(thiophen-2-yl)hexa-4,5-dienoate (3p) - Was synthesized according to general procedure $2 \mathrm{~b}$ using $1 \mathbf{p}(66.5 \mathrm{mg}, 0.4 \mathrm{mmol})$, $\left[\operatorname{Ir}(\operatorname{cod}) \mathrm{Cl}_{2}(13.4 \mathrm{mg}, 20 \mu \mathrm{mol}, 5.0 \mathrm{~mol} \%),(S)-\mathbf{L} \mathbf{1}(40.6 \mathrm{mg}, 80 \mu \mathrm{mol}, 20\right.$ mol\%), 2a (265 $\mu \mathrm{L}, 1.0 \mathrm{mmol}, 2.5$ equiv), and La(OTf) 3 (17.6 mg, $30 \mu \mathrm{mol}$, $7.5 \mathrm{~mol} \%$ ), and the reaction was run for 2 hours. The regiomeric ratio (rr) was determined to be 7:1 by ${ }^{1} \mathrm{H}$ NMR analysis of the unpurified reaction mixture. The title compound was obtained as a mixture with inseparable 1,3-diene isomer by purification via $\mathrm{SiO}_{2}$ flash column chromatography using pentane: $\mathrm{Et}_{2} \mathrm{O}(50: 1$ to $30: 1 \mathrm{v}: \mathrm{v})$. The title compound was obtained as a clear colorless oil (74.4 mg, $0.28 \mathrm{mmol}, 70 \%$ yield). ${ }^{1} \mathbf{H}$ NMR $\left(500 \mathrm{MHz}, \mathrm{CDCl}_{3}\right) \delta 7.15$ (dd, $J=5.1,1.2 \mathrm{~Hz}, 1 \mathrm{H}), 6.93(\mathrm{dd}, J=5.1,3.6 \mathrm{~Hz}, 1 \mathrm{H}), 6.89(\mathrm{dd}, J=3.6,1.2 \mathrm{~Hz}, 1 \mathrm{H}), 6.00(\mathrm{td}, J=$ 6.7, $0.6 \mathrm{~Hz}, 1 \mathrm{H}), 4.86-4.75(\mathrm{~m}, 2 \mathrm{H}), 4.00(\mathrm{q}, J=7.1 \mathrm{~Hz}, 2 \mathrm{H}), 1.58(\mathrm{~d}, J=0.6 \mathrm{~Hz}, 3 \mathrm{H}), 1.24$ $(\mathrm{s}, 3 \mathrm{H}), 1.22(\mathrm{~s}, 3 \mathrm{H}), 1.15(\mathrm{t}, J=7.1 \mathrm{~Hz}, 3 \mathrm{H}) \cdot{ }^{13} \mathbf{C} \mathbf{N M R}\left(125 \mathrm{MHz}, \mathrm{CDCl}_{3}\right) \delta 207.5,176.0$, 149.7, 126.0, 124.9, 123.2, 97.0, 77.6, 60.4, 50.0, 45.5, 23.4, 22.5, 22.2, 14.0. IR (atr, $\left.\mathrm{cm}^{-1}\right)$ 2980, 2938, 1956, 1718, 1463, 1379, 1266, 1173, 1135, 1085, 1026, 849, 696. HRMS (ESI+, $\mathrm{M}+\mathrm{Na}$ ) Calc'd for $\mathrm{C}_{15} \mathrm{H}_{20} \mathrm{O}_{2} \mathrm{SNa}$ 287.1076, found 287.1073. Enantiomeric purity was determined by HPLC analysis in comparison with racemic material $\mathrm{T}_{\mathrm{R}}=5.3$ (minor) $\mathrm{T}_{\mathrm{R}}=7.0$ (major) (96.5:3.5 er shown); Chiracel IC column, 99\% hexane to $1 \% \mathrm{iPrOH}, 1.0 \mathrm{~mL} / \mathrm{min}, 240$ $\left.\mathrm{nm}, 480 \mathrm{psi}, 2{ }^{\circ} \mathrm{C}\right)[\boldsymbol{\alpha}]_{\boldsymbol{D}}^{26}=-64.3\left(\mathrm{c}=0.5, \mathrm{CHCl}_{3}\right)$. The absolute stereochemistry of this compound was assigned in analogy to $\mathbf{3 a}$.
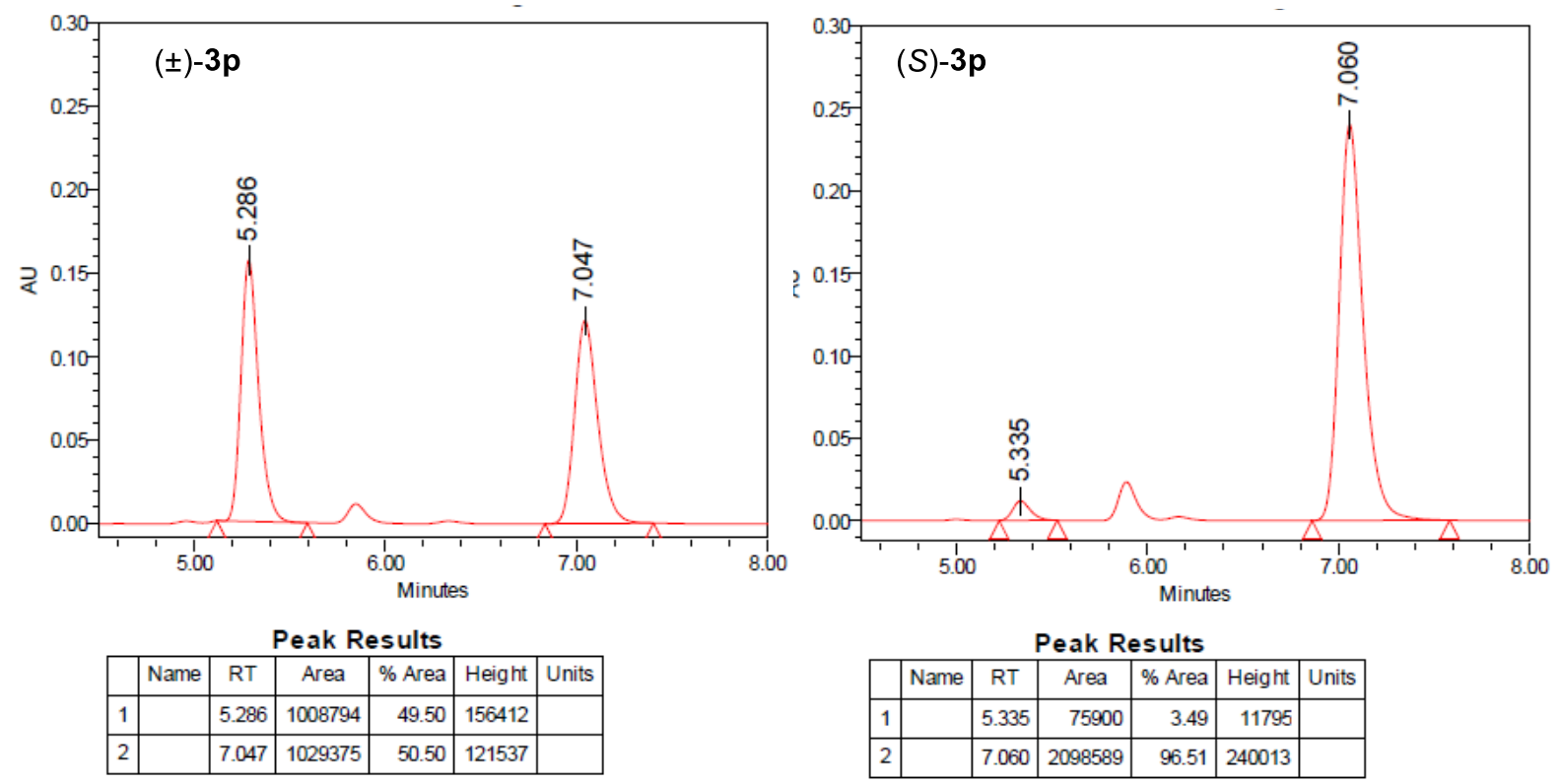

Characterization data for 1,3-diene byproduct 3p':

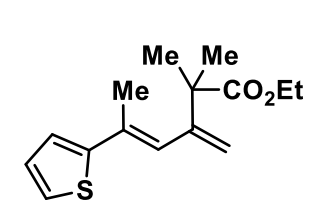

${ }^{1} \mathbf{H}$ NMR $\left(500 \mathrm{MHz} \mathrm{CDCl}_{3}\right) \delta 7.15-7.14(\mathrm{~m}, 1 \mathrm{H}), 7.04(\mathrm{dd}, J=3.6,1.2$ $\mathrm{Hz}, 1 \mathrm{H}), 6.98(\mathrm{dd}, J=5.1,3.6 \mathrm{~Hz}, 1 \mathrm{H}), 6.40(\mathrm{t}, J=1.4 \mathrm{~Hz}, 1 \mathrm{H}), 5.30(\mathrm{~d}, J$ $=1.1 \mathrm{~Hz}, 1 \mathrm{H}), 5.01(\mathrm{dd}, J=1.5,1.1 \mathrm{~Hz}, 1 \mathrm{H}), 4.13(\mathrm{q}, J=7.1 \mathrm{~Hz}, 2 \mathrm{H}), 2.17$ $(\mathrm{d}, J=1.4 \mathrm{~Hz}, 3 \mathrm{H}), 1.37(\mathrm{~s}, 6 \mathrm{H}), 1.23(\mathrm{t}, J=7.1 \mathrm{~Hz}, 3 \mathrm{H})$.

${ }^{13} \mathrm{C}$ NMR $\left(125 \mathrm{MHz}, \mathrm{CDCl}_{3}\right) \delta 176.1,148.3,147.6,132.0,127.3,123.8$, 123.3, 113.6, 112.7, 60.7, 47.7, 24.8 (2C), 17.3, 14.2. 


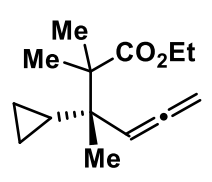

ethyl (S)-3-cyclopropyl-2,2,3-trimethylhexa-4,5-dienoate (3q) - Was synthesized according to general procedure $2 \mathrm{~b}$ using $1 \mathrm{q}(49.7 \mathrm{mg}, 0.4 \mathrm{mmol})$, $[\operatorname{Ir}(\mathrm{cod}) \mathrm{Cl}]_{2}(13.4 \mathrm{mg}, 20 \mu \mathrm{mol}, 5.0 \mathrm{~mol} \%),(S)-\mathbf{L} \mathbf{1}(40.6 \mathrm{mg}, 80 \mu \mathrm{mol}, 20 \mathrm{~mol} \%)$, 2a $\left(265 \mu \mathrm{L}, 1.0 \mathrm{mmol}, 2.5\right.$ equiv), and $\mathrm{La}(\mathrm{OTf})_{3}(17.6 \mathrm{mg}, 30 \mu \mathrm{mol}, 7.5 \mathrm{~mol} \%)$, and the reaction was run at $23{ }^{\circ} \mathrm{C}$ for 22 hours. The regiomeric ratio (rr) was determined to be $>20: 1$ by ${ }^{1} \mathrm{H}$ NMR analysis of the unpurified reaction mixture. The title compound was obtained by purification via $\mathrm{SiO}_{2}$ flash column chromatography using pentane:Et $\mathrm{O}_{2} \mathrm{O}$ (100:1 to 50:1 v:v). The title compound was obtained as a clear colourless oil (48.3 mg, $0.22 \mathrm{mmol}, 54 \%$ yield). ${ }^{1} \mathbf{H}$ NMR $\left(400 \mathrm{MHz}, \mathrm{CDCl}_{3}\right) \delta 5.12(\mathrm{t}, J=6.7 \mathrm{~Hz}, 1 \mathrm{H}), 4.76-4.66(\mathrm{~m}, 2 \mathrm{H}), 4.15-4.07(\mathrm{~m}, 2 \mathrm{H})$, 1.26 (t, $J=7.2 \mathrm{~Hz}, 3 \mathrm{H}), 1.23$ (s, 6H), $1.03(\mathrm{tt}, J=8.4,5.6 \mathrm{~Hz}, 1 \mathrm{H}), 0.94(\mathrm{~s}, 3 \mathrm{H}), 0.38-0.19$ (m, $3 \mathrm{H}), 0.15-0.07(\mathrm{~m}, 1 \mathrm{H}) .{ }^{13} \mathbf{C} \mathbf{N M R}\left(100 \mathrm{MHz}, \mathrm{CDCl}_{3}\right) \delta 208.5,176.8,92.9,75.9,60.2,49.3$, 41.8, 22.1, 21.8, 19.2, 17.2, 14.2, 1.9, 0.5. IR (atr, $\left.\mathrm{cm}^{-1}\right)$ 3080, 2979, 1954, 1720, 1462, 1380, $1265,1173,1142,1097,1022,948,839,769$. HRMS (ESI+, M+Na) Calc'd for $\mathrm{C}_{14} \mathrm{H}_{22} \mathrm{O}_{2} \mathrm{Na}$ 245.1512 , found 245.1514. Enantiomeric purity was determined by GC analysis in comparison with racemic material $\mathrm{T}_{\mathrm{R}}=202.1$ (minor) $\mathrm{T}_{\mathrm{R}}=204.9$ (major) (97:3 er shown); Agilent CP-ChiDEX-CB; $25 \mathrm{~m}, 0.25 \mathrm{~mm}, 0.25 \mu \mathrm{m}$ (Intuvo) $70{ }^{\circ} \mathrm{C}, 2.5 \mathrm{~mL} / \mathrm{min} .[\boldsymbol{\alpha}]_{\boldsymbol{D}}^{\mathbf{2 6}}=-21.8(\mathrm{c}=0.45$, $\left.\mathrm{CHCl}_{3}\right)$. The absolute stereochemistry of this compound was assigned by $\mathrm{X}$-ray crystallographic analysis of a derivative $(S)$-S8 (vide infra).

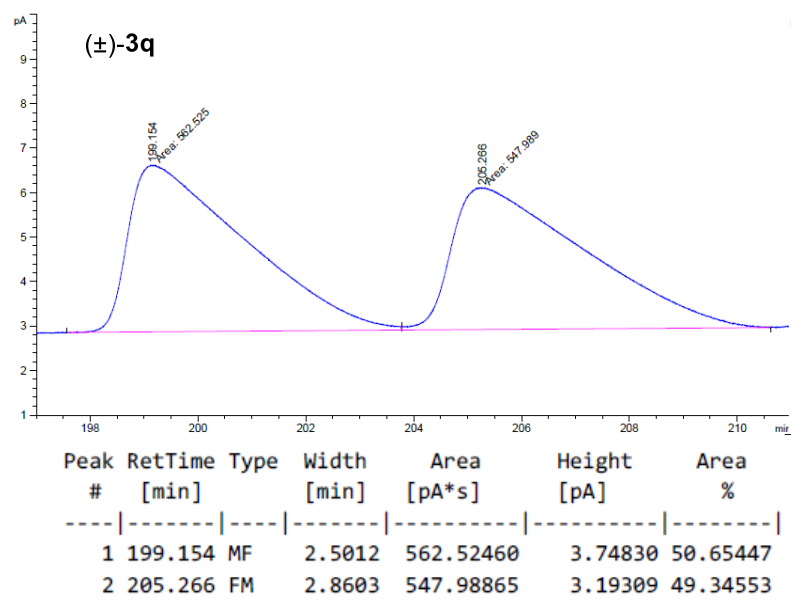

$(S)-3 q$

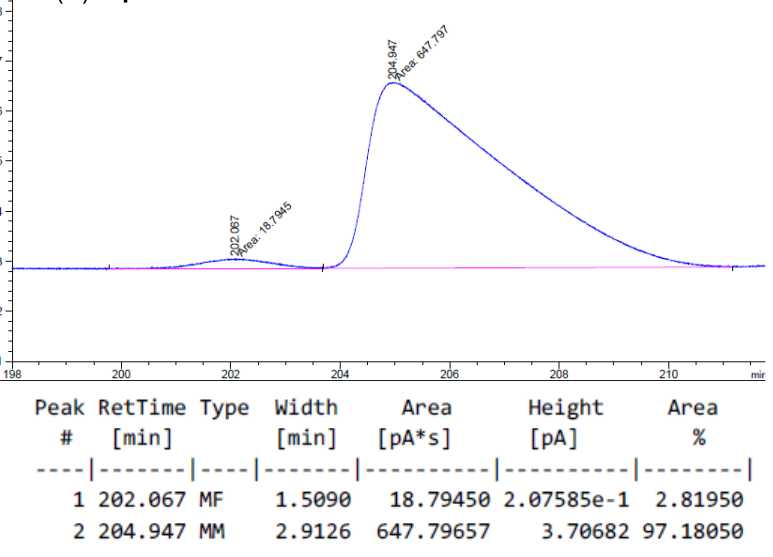




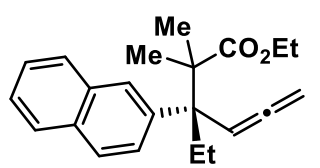

ethyl $(R)$-3-ethyl-2,2-dimethyl-3-(naphthalen-2-yl)hexa-4,5-dienoate

(3s) - Was synthesized according to general procedure 2a using 1s $(89.7$ $\mathrm{mg}, 0.4 \mathrm{mmol}),[\mathrm{Ir}(\mathrm{cod}) \mathrm{Cl}]_{2}(13.4 \mathrm{mg}, 20 \mu \mathrm{mol}, 5.0 \mathrm{~mol} \%),(S)-\mathbf{L} \mathbf{1}(40.6$ $\mathrm{mg}, 80 \mu \mathrm{mol}, 20 \mathrm{~mol} \%), \mathbf{2 b}\left(222 \mu \mathrm{L}, 1.0 \mathrm{mmol}, 2.5\right.$ equiv), and $\mathrm{La}(\mathrm{OTf})_{3}$ (17.6 mg, $30 \mu \mathrm{mol}, 7.5 \mathrm{~mol} \%$ ), and the reaction was run for 53 hours. The regiomeric ratio (rr) was determined to be $>20: 1$ by ${ }^{1} \mathrm{H}$ NMR analysis of the unpurified reaction mixture. The title compound was obtained by purification via $\mathrm{SiO}_{2}$ flash column chromatography using pentane:Et ${ }_{2} \mathrm{O}(70: 1 \mathrm{v}: \mathrm{v})$. The title compound was obtained as a clear colourless oil $(67.6 \mathrm{mg}$, $0.21 \mathrm{mmol}, 52 \%$ yield). ${ }^{1} \mathbf{H}$ NMR $\left(400 \mathrm{MHz}, \mathrm{CDCl}_{3}\right) \delta 7.85-7.73(\mathrm{~m}, 4 \mathrm{H}), 7.50-7.43(\mathrm{~m}$, $3 \mathrm{H}), 5.96(\mathrm{td}, J=6.8,1.3 \mathrm{~Hz}, 1 \mathrm{H}), 4.84-4.72(\mathrm{~m}, 2 \mathrm{H}), 4.05-3.88(\mathrm{~m}, 2 \mathrm{H}), 2.55(\mathrm{dq}, J=14.4$, $7.1 \mathrm{~Hz}, 1 \mathrm{H}), 1.95(\mathrm{dq}, J=14.4,7.2 \mathrm{~Hz}, 1 \mathrm{H}), 1.21(\mathrm{~s}, 3 \mathrm{H}), 1.13(\mathrm{~s}, 3 \mathrm{H}), 1.05(\mathrm{t}, J=7.1 \mathrm{~Hz}, 3 \mathrm{H})$, $0.74(\mathrm{t}, J=7.2 \mathrm{~Hz}, 3 \mathrm{H}) .{ }^{13} \mathbf{C}$ NMR $\left(100 \mathrm{MHz}, \mathrm{CDCl}_{3}\right) \delta 208.8,176.4,137.6,132.7,131.9$, 128.6, 128.1, 127.6, 127.2, 126.2, 125.7, 125.7, 92.8, 76.6, 60.3, 52.4, 50.8, 25.4, 22.7, 22.5, 13.8, 10.0. IR (atr, $\left.\mathrm{cm}^{-1}\right)$ 3057, 2977, 2937, 2879, 1955, 1715, 1598, 1506, 1469, 1386, 1367, $1260,1169,1138,1027,857,841,816,745,478$. HRMS (ESI+, M+Na) Calc'd for $\mathrm{C}_{22} \mathrm{H}_{26} \mathrm{O}_{2} \mathrm{Na}$ 345.1825 , found 345.1827 . Enantiomeric purity was determined by SFC analysis in comparison with racemic material $\mathrm{T}_{\mathrm{R}}=9.1$ (minor) $\mathrm{T}_{\mathrm{R}}=16.1$ (major) (95:5 er shown); Chiracel OJ-H column, $90 \% \mathrm{CO}_{2}$ to $\left.10 \% \mathrm{MeOH}, 2.0 \mathrm{~mL} / \mathrm{min}, 222 \mathrm{~nm}, 126 \mathrm{bar}, 25^{\circ} \mathrm{C}\right)[\boldsymbol{\alpha}]_{\boldsymbol{D}}^{24}=-109.7(\mathrm{c}=$ $\left.0.5, \mathrm{CHCl}_{3}\right)$. The absolute stereochemistry of this compound was assigned in analogy to $\mathbf{3 a}$.
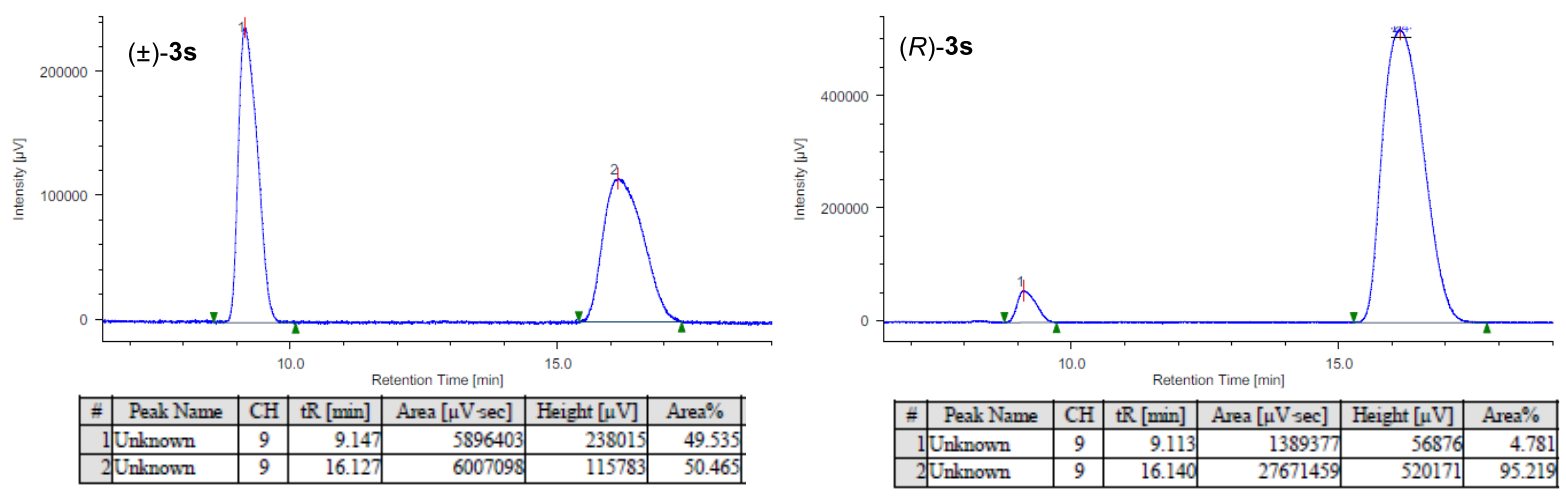


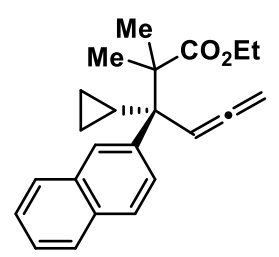

ethyl

(S)-3-cyclopropyl-2,2-dimethyl-3-(naphthalen-2-yl)hexa-4,5-

dienoate (3t) - Was synthesized according to general procedure $2 \mathrm{~b}$ using $\mathbf{1 t}$ (94.5 mg, $0.4 \mathrm{mmol}),\left[\operatorname{Ir}(\operatorname{cod}) \mathrm{Cl}_{2}(13.4 \mathrm{mg}, 20 \mu \mathrm{mol}, 5.0 \mathrm{~mol} \%),(S)-\mathbf{L}_{1}\right.$ (40.6 mg, $80 \mu \mathrm{mol}, 20 \mathrm{~mol} \%), 2 \mathbf{b}(222 \mu \mathrm{L}, 1.0 \mathrm{mmol}, 2.5$ equiv), and $\mathrm{La}(\mathrm{OTf})_{3}(17.6 \mathrm{mg}, 30 \mu \mathrm{mol}, 7.5 \mathrm{~mol} \%)$, and the reaction was run for 44 hours. The regiomeric ratio (rr) was determined to be $>20: 1$ by ${ }^{1} \mathrm{H}$ NMR analysis of the unpurified reaction mixture. The title compound was obtained by purification via $\mathrm{SiO}_{2}$ flash column chromatography using pentane: $\mathrm{Et}_{2} \mathrm{O}(50: 1 \mathrm{v}: \mathrm{v})$. The title compound was obtained as a clear colourless oil (73.1 mg, $0.22 \mathrm{mmol}, 55 \%$ yield). ${ }^{1} \mathbf{H} \mathbf{~ N M R}$ (400 MHz, $\mathrm{CDCl}_{3}$ ) $\delta 7.99-7.95(\mathrm{~m}, 1 \mathrm{H}), 7.86-7.78(\mathrm{~m}, 2 \mathrm{H}), 7.75-7.69(\mathrm{~m}, 1 \mathrm{H}), 7.67-7.60(\mathrm{~m}, 1 \mathrm{H}), 7.50-$ $7.40(\mathrm{~m}, 2 \mathrm{H}), 5.75(\mathrm{t}, J=6.8 \mathrm{~Hz}, 1 \mathrm{H}), 4.63-4.38(\mathrm{~m}, 2 \mathrm{H}), 4.15-3.98(\mathrm{~m}, 2 \mathrm{H}), 1.85(\mathrm{tt}, J=$ 8.1, $5.7 \mathrm{~Hz}, 1 \mathrm{H}), 1.37(\mathrm{~s}, 3 \mathrm{H}), 1.18(\mathrm{t}, J=7.1 \mathrm{~Hz}, 3 \mathrm{H}), 1.01(\mathrm{~s}, 3 \mathrm{H}), 0.65-0.50(\mathrm{~m}, 3 \mathrm{H}), 0.13$ $-0.03(\mathrm{~m}, 1 \mathrm{H}) .{ }^{13} \mathbf{C ~ N M R}\left(100 \mathrm{MHz}, \mathrm{CDCl}_{3}\right) \delta 209.0,176.5,140.5,132.6,132.1,129.0,128.6$, $128.3,127.2$, 125.7, 125.6, 125.5, 90.4, 76.7, 60.5, 52.2, 50.0, 23.2, 22.6, 16.1, 14.0, 4.3, 1.0. IR $\left(\mathrm{atr}, \mathrm{cm}^{-1}\right)$ 3057, 2977, 2928, 1954, 1714, 1597, 1505, 1467, 1388, 1262, 1174, 1138, 1120 , 1027, 898, 857, 816, 745, 478. HRMS (ESI+, M+Na) Calc'd for $\mathrm{C}_{23} \mathrm{H}_{26} \mathrm{O}_{2} \mathrm{Na} 357.1825$, found 357.1823. Enantiomeric purity was determined by SFC analysis in comparison with racemic material $\mathrm{T}_{\mathrm{R}}=9.4$ (major) $\mathrm{T}_{\mathrm{R}}=14.4$ (major) (97:3 er shown); Chiracel OJ-H column, $88 \% \mathrm{CO}_{2}$ to $2 \% \mathrm{MeOH}, 2.0 \mathrm{~mL} / \mathrm{min}, 226 \mathrm{~nm}, 130$ bar, $\left.25^{\circ} \mathrm{C}\right)[\alpha]_{D}^{25}=+20.5\left(\mathrm{c}=0.5, \mathrm{CHCl}_{3}\right)$. The absolute stereochemistry of this compound was assigned by X-ray crystallographic analysis of a derivative (S)-S10 (vide infra).
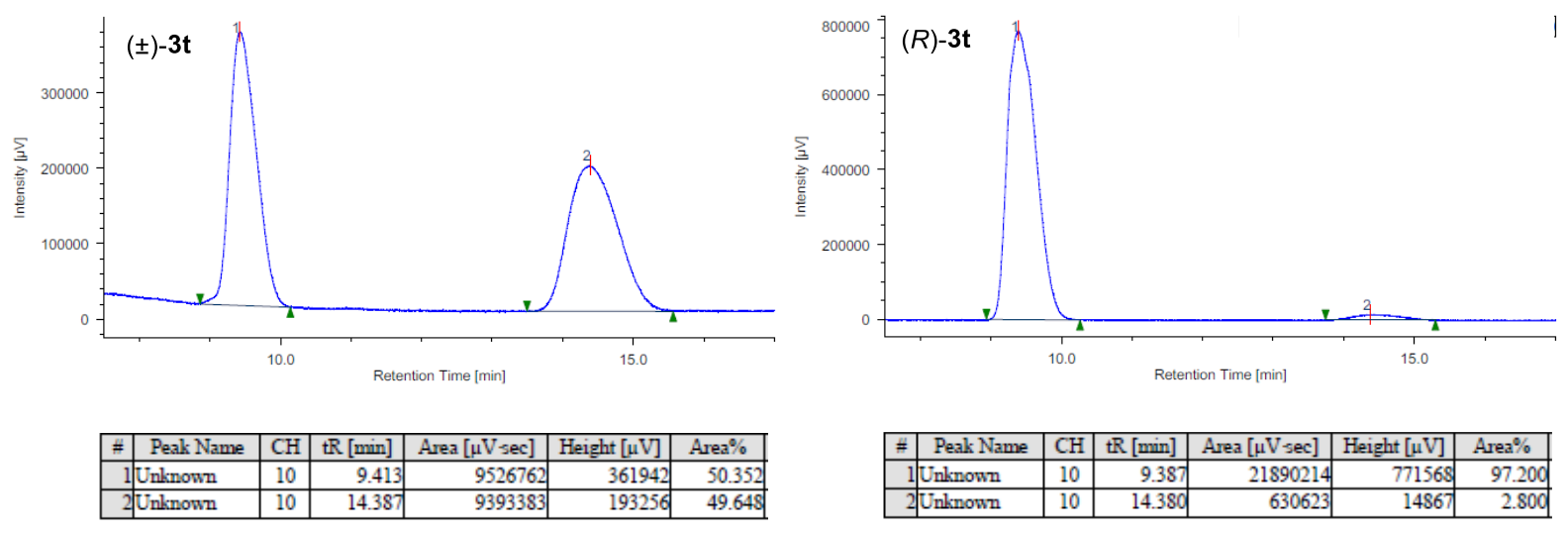


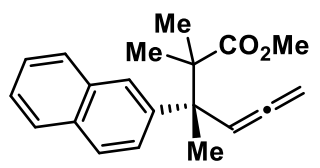

methyl $(R)-2,2,3-$ trimethyl-3-(naphthalen-2-yl)hexa-4,5-dienoate (3u)

- Was synthesized according to general procedure 2a using $1 \mathbf{a}(89.7 \mathrm{mg}$, $0.4 \mathrm{mmol}),\left[\mathrm{Ir}(\mathrm{cod}) \mathrm{Cl}_{2}(13.4 \mathrm{mg}, 20 \mu \mathrm{mol}, 5.0 \mathrm{~mol} \%),(S)-\mathbf{L} \mathbf{1}(40.6 \mathrm{mg}\right.$, $80 \mu \mathrm{mol}, 20 \mathrm{~mol} \%), \mathbf{2 d}(246 \mu \mathrm{L}, 1.0 \mathrm{mmol}, 2.5 \text { equiv), and La(OTf })_{3}(23.4$ $\mathrm{mg}, 40 \mu \mathrm{mol}, 10 \mathrm{~mol} \%$ ), and the reaction was run for 36 hours. The regiomeric ratio (rr) was determined to be $>20: 1$ by ${ }^{1} \mathrm{H}$ NMR analysis of the unpurified reaction mixture. The title compound was obtained by purification via $\mathrm{SiO}_{2}$ flash column chromatography using pentane: $\mathrm{Et}_{2} \mathrm{O}(40: 1$ to $20: 1 \mathrm{v}: \mathrm{v})$. The title compound was obtained as a clear slightly yellow oil (82.4 mg, $0.28 \mathrm{mmol}, 70 \%$ yield). ${ }^{1} \mathbf{H}$ NMR $\left(400 \mathrm{MHz}, \mathrm{CDCl}_{3}\right) \delta 7.86-7.71(\mathrm{~m}, 4 \mathrm{H}), 7.53-$ $7.40(\mathrm{~m}, 3 \mathrm{H}), 6.25(\mathrm{t}, J=6.7 \mathrm{~Hz}, 1 \mathrm{H}), 4.84-4.66(\mathrm{~m}, 2 \mathrm{H}), 3.50(\mathrm{~s}, 3 \mathrm{H}), 1.66(\mathrm{~s}, 3 \mathrm{H}), 1.21(\mathrm{~s}$, 6H). ${ }^{13} \mathbf{C}$ NMR $\left(100 \mathrm{MHz}, \mathrm{CDCl}_{3}\right) \delta 207.7,176.8,142.0,132.8,131.9,128.1,127.2,126.8$, 126.5(2C), 125.8, 125.7, 96.6, 77.1, 51.4, 49.9, 46.6, 22.4, 22.2, 22.1. IR $\left(\right.$ atr, $\left.\mathrm{cm}^{-1}\right) 3057,2979$, 2947, 1954, 1721, 1598, 1506, 1464, 1432, 1380, 1392, 1267, 1189, 1139, 1126, 1087, 985, 948, 850, 818, 745, 477. HRMS (ESI+, M+K) Calc'd for $\mathrm{C}_{20} \mathrm{H}_{22} \mathrm{O}_{2} \mathrm{~K}$ 333.1251, found 333.1254. Enantiomeric purity was determined by SFC analysis in comparison with racemic material $\mathrm{T}_{\mathrm{R}}=7.7$ (major) $\mathrm{T}_{\mathrm{R}}=8.9$ (minor) (97.5:2.5 er shown); Chiracel OB-H column, 95\% $\mathrm{CO}_{2}$ to $\left.5 \% \mathrm{MeOH}, 2.0 \mathrm{~mL} / \mathrm{min}, 223 \mathrm{~nm}, 121 \mathrm{bar}, 25^{\circ} \mathrm{C}\right)[\alpha]_{D}^{24}=-160.3\left(\mathrm{c}=0.5, \mathrm{CHCl}_{3}\right)$. The absolute stereochemistry of this compound was assigned in analogy to $\mathbf{3 a}$.
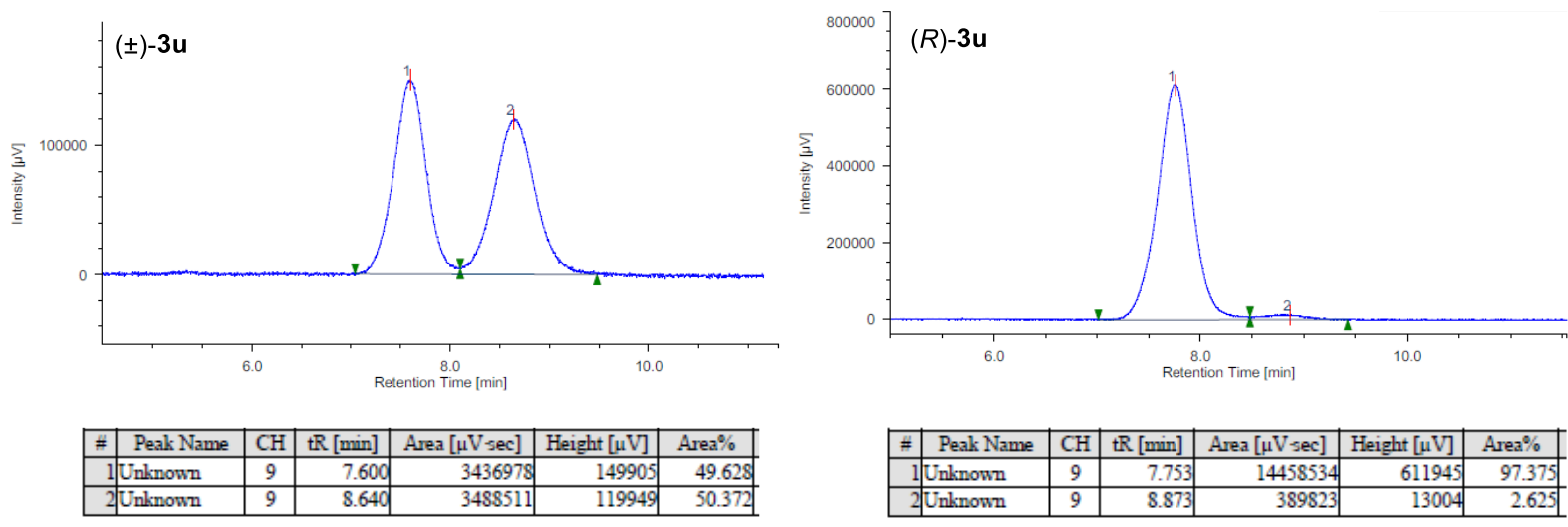


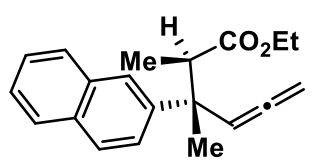

ethyl $\quad(2 R, 3 S)-2,3-d i m e t h y l-3-(n a p h t h a l e n-2-y l) h e x a-4,5-d i e n o a t e$

$[(R, S)-3 \mathbf{v}]$ - Was synthesized according to general procedure 2a using 1a (89.7 mg, $0.4 \mathrm{mmol}),[\operatorname{Ir}(\mathrm{cod}) \mathrm{Cl}]_{2}(13.4 \mathrm{mg}, 20 \mu \mathrm{mol}, 5.0 \mathrm{~mol} \%),(S)-\mathbf{L} \mathbf{1}$ (40.6 mg, $80 \mu \mathrm{mol}, 20 \mathrm{~mol} \%),(E)-2 \mathrm{e}(250 \mu \mathrm{L}, 1.0 \mathrm{mmol}, 2.5$ equiv), and $\mathrm{La}(\mathrm{OTf})_{3}(17.6 \mathrm{mg}, 30 \mu \mathrm{mol}, 7.5 \mathrm{~mol} \%)$, and the reaction was run for 36 hours. The regiomeric ratio (rr) was determined to be $>20: 1$ and the diastereomeric ratio (dr) was determined to be 2.4:1 by ${ }^{1} \mathrm{H}$ NMR analysis of the unpurified reaction mixture. The title compound was obtained by purification via $\mathrm{SiO}_{2}$ flash column chromatography using pentane:Et $\mathrm{t}_{2} \mathrm{O}$ (50:1 to $33: 1 \mathrm{v}: \mathrm{v}$ ) The title compound was obtained as a clear colorless oil which solidified to a white solid upon standing at $-20{ }^{\circ} \mathrm{C}\left(63.3 \mathrm{mg}, 0.22 \mathrm{mmol}, 54 \%\right.$ yield, $\left.\mathrm{MP}=61.9-62.5^{\circ} \mathrm{C}\right) .{ }^{1} \mathbf{H}$ NMR $(400 \mathrm{MHz}$, $\left.\mathrm{CDCl}_{3}\right) \delta 7.87-7.74(\mathrm{~m}, 4 \mathrm{H}), 7.63-7.53(\mathrm{~m}, 1 \mathrm{H}), 7.49-7.38(\mathrm{~m}, 2 \mathrm{H}), 5.95(\mathrm{t}, J=6.6 \mathrm{~Hz}$, $1 \mathrm{H}), 4.93-4.74(\mathrm{~m}, 2 \mathrm{H}), 3.94(\mathrm{qd}, J=7.1,1.8 \mathrm{~Hz}, 2 \mathrm{H}), 3.16(\mathrm{q}, J=7.1 \mathrm{~Hz}, 1 \mathrm{H}), 1.56(\mathrm{~s}, 3 \mathrm{H})$, $1.13(\mathrm{~d}, J=7.1 \mathrm{~Hz}, 3 \mathrm{H}), 1.01(\mathrm{t}, J=7.1 \mathrm{~Hz}, 3 \mathrm{H}) .{ }^{13} \mathbf{C} \mathbf{N M R}\left(100 \mathrm{MHz}, \mathrm{CDCl}_{3}\right) \delta 207.8,174.8$, 144.0, 133.1, 132.0, 128.0, 127.6, 127.3, 125.9, 125.6, 125.1, 125.0, 96.9, 77.1, 59.9, 48.9, 44.1, 23.2, 14.0, 13.5. IR (atr, $\left.\mathrm{cm}^{-1}\right)$ 3057, 2978, 2936, 1955, 1728, 1598, 1505, 1453, 1371, 1339, 1259, 1195, 1150, 1129, 1082, 1020, 853, 816, 746, 477. HRMS (ESI+, M+K) Calc'd for $\mathrm{C}_{20} \mathrm{H}_{22} \mathrm{O}_{2} \mathrm{~K} 333.1251$, found 333.1257. Enantiomeric purity was determined by HPLC analysis in comparison with racemic material $\mathrm{T}_{\mathrm{R}}=6.1$ (minor) $\mathrm{T}_{\mathrm{R}}=6.8$ (major) (92.5:7.5 er shown); Chiracel IC column, 99\% hexane to $\left.1 \% \mathrm{iPrOH}, 1.0 \mathrm{~mL} / \mathrm{min}, 221 \mathrm{~nm}, 495 \mathrm{psi}, 25^{\circ} \mathrm{C}\right)[\boldsymbol{\alpha}]_{\boldsymbol{D}}^{\mathbf{2 6}}=-$ $155.8\left(\mathrm{c}=0.5, \mathrm{CHCl}_{3}\right)$. The absolute and relative stereochemistry of this compound was assigned by X-ray crystallographic analysis of a derivative $(R, S)$-S12 and noe measurement of a derivative $(R, S)$-S13 (vide infra).
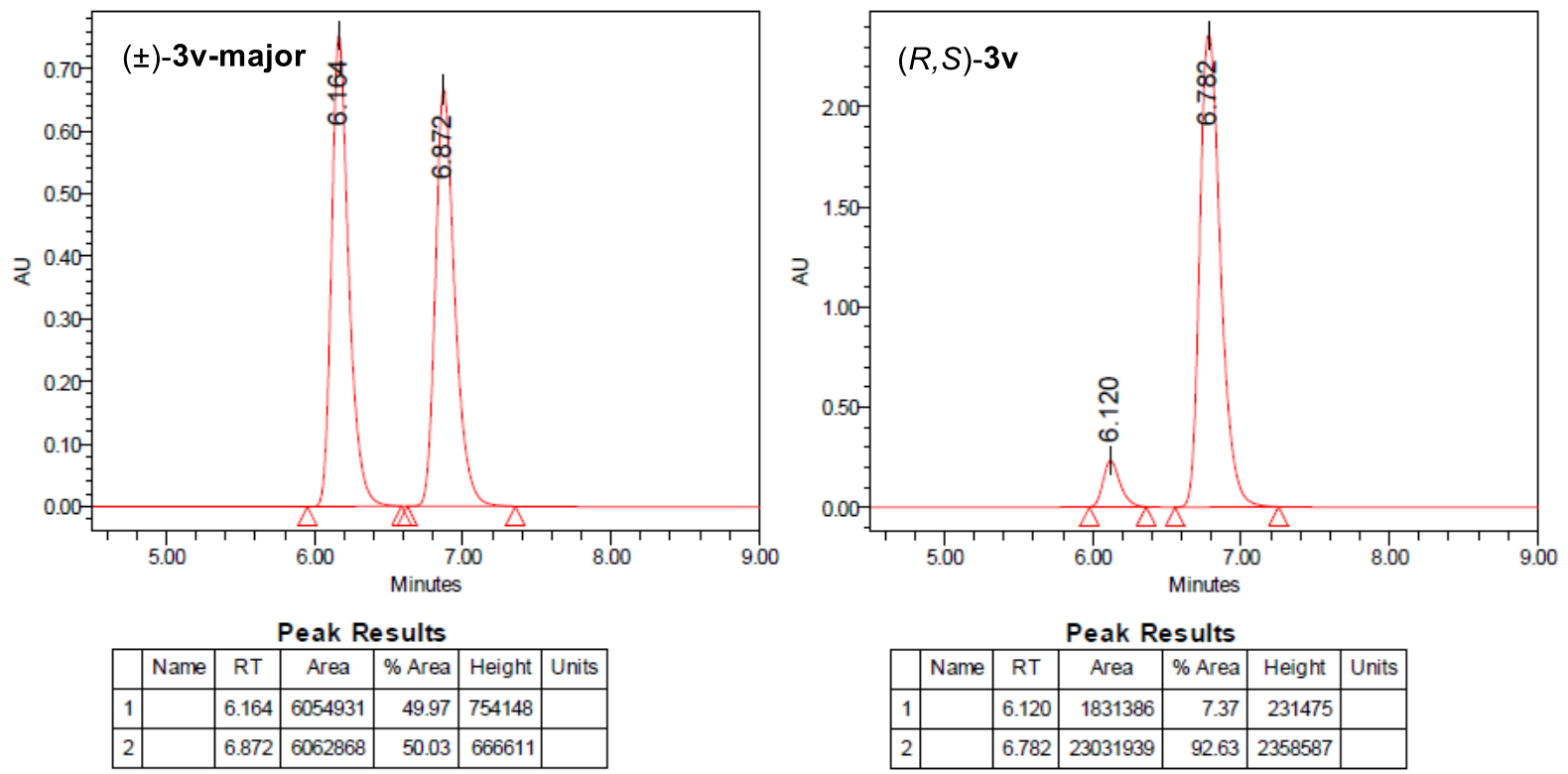

Characterization data for the minor diastereomer $(S, S)-3 \mathrm{v}$ :

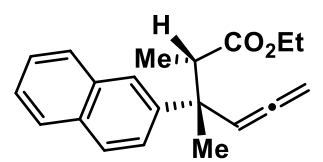

ethyl

(2S,3S)-2,3-dimethyl-3-(naphthalen-2-yl)hexa-4,5-dienoate

$[(S, S)-3 \mathbf{v}]-$ The title compound was obtained as a colorless oil $(21.4 \mathrm{mg}$, $0.07 \mathrm{mmol}, 18 \%$ yield $)^{\mathbf{1}} \mathbf{H}$ NMR $\left(400 \mathrm{MHz}, \mathrm{CDCl}_{3}\right) \delta 7.85-7.73(\mathrm{~m}$, 4H), $7.60-7.53(\mathrm{~m}, 1 \mathrm{H}), 7.50-7.38(\mathrm{~m}, 2 \mathrm{H}), 5.57(\mathrm{t}, J=6.7 \mathrm{~Hz}, 1 \mathrm{H}), 4.98-4.81(\mathrm{~m}, 2 \mathrm{H})$, $3.88-3.73(\mathrm{~m}, 2 \mathrm{H}), 3.12(\mathrm{q}, J=7.0 \mathrm{~Hz}, 1 \mathrm{H}), 1.63(\mathrm{~s}, 3 \mathrm{H}), 1.16(\mathrm{~d}, J=7.0 \mathrm{~Hz}, 3 \mathrm{H}), 0.85(\mathrm{t}, J$ $=7.1 \mathrm{~Hz}, 3 \mathrm{H}) .{ }^{13} \mathbf{C} \mathbf{N M R}\left(100 \mathrm{MHz}, \mathrm{CDCl}_{3}\right) \delta 207.7,174.5,143.6,133.1,132.0,128.1,127.6$, 
127.3, 125.9, 125.7, 125.2, 125.0, 97.1, 77.6, 59.9, 48.8, 44.2, 22.4, 13.8, 13.4. IR (atr, $\left.\mathrm{cm}^{-1}\right)$ 3057, 2979. 2935, 1955, 1728, 1631, 1599, 1505, 1455, 1375, 1339, 1259, 1194, 1155, 1129, 1096, 1070, 1044, 1019, 949, 853, 815, 746, 477. HRMS (ESI+, M+Na) Calc'd for $\mathrm{C}_{20} \mathrm{H}_{22} \mathrm{O}_{2} \mathrm{Na}$ 317.1512, found 317.1510. Enantiomeric purity was determined by HPLC analysis in comparison with racemic material $\mathrm{T}_{\mathrm{R}}=6.2$ (major) $\mathrm{T}_{\mathrm{R}}=6.8$ (minor) (91:9 er shown); Chiracel IC column, $99 \%$ hexane to $\left.1 \% \mathrm{iPrOH}, 1.0 \mathrm{~mL} / \mathrm{min}, 225 \mathrm{~nm}, 495 \mathrm{psi}, 25^{\circ} \mathrm{C}\right)[\boldsymbol{\alpha}]_{\boldsymbol{D}}^{\mathbf{2 5}}=+6.2(\mathrm{c}=$ $\left.0.5, \mathrm{CHCl}_{3}\right)$.
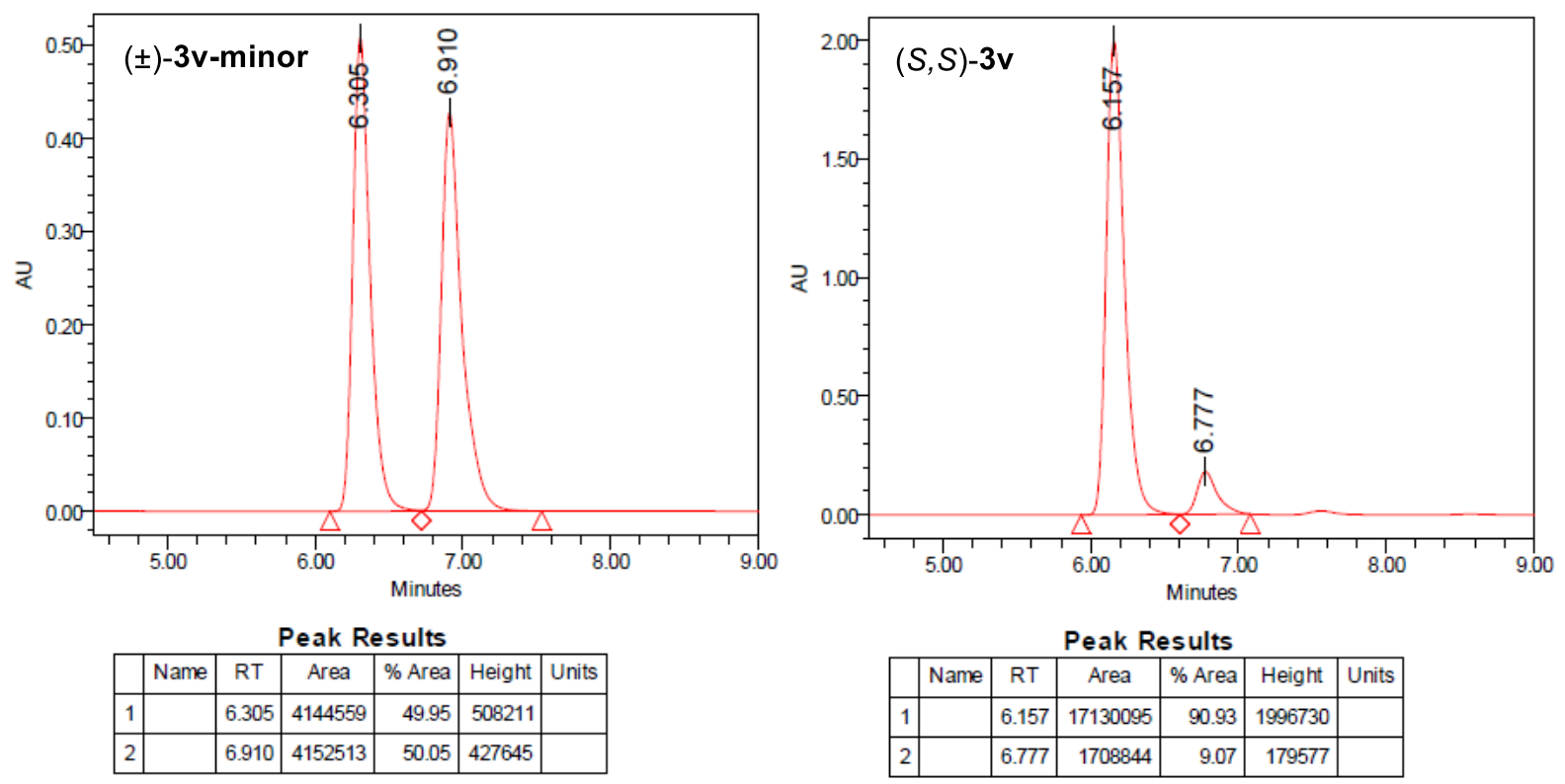


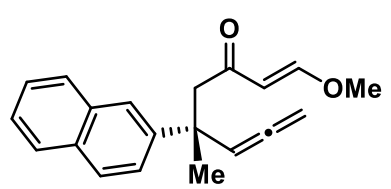

$(R, E)$-1-methoxy-5-methyl-5-(naphthalen-2-yl)octa-1,6,7-trien-3one $[(R, E)-3 \mathbf{w}]-$ Was synthesized according to general procedure $2 \mathrm{a}$ using 1a $(89.7 \mathrm{mg}, 0.4 \mathrm{mmol})$, [ $\mathrm{Ir}(\mathrm{cod}) \mathrm{Cl}]_{2}(13.4 \mathrm{mg}, 20 \mu \mathrm{mol}, 5.0$ mol\%), (S)-L 1 (40.6 mg, $80 \mu \mathrm{mol}, 20 \mathrm{~mol} \%), 2 f(194 \mu \mathrm{L}, 1.0 \mathrm{mmol}$, 2.5 equiv), and $\mathrm{La}(\mathrm{OTf})_{3}(7.0 \mathrm{mg}, 12 \mu \mathrm{mol}, 3.0 \mathrm{~mol} \%)$, and the reaction was run for 78 hours. The regiomric ratio (rr) was determined to be $>20: 1$ by ${ }^{1} \mathrm{H}$ NMR analysis of the unpurified reaction mixture. The title compound was obtained by purification via $\mathrm{SiO}_{2}$ flash column chromatography using pentane:Et $\mathrm{t}_{2} \mathrm{O}(5: 1 \mathrm{v}: \mathrm{v})$ The title compound was obtained as a clear yellow oil (63.4 mg, $0.22 \mathrm{mmol}, 54 \%$ yield). ${ }^{1} \mathbf{H} \mathbf{~ N M R}\left(400 \mathrm{MHz}, \mathrm{CDCl}_{3}\right) \delta 7.85-7.74(\mathrm{~m}, 4 \mathrm{H})$, $7.62-7.51(\mathrm{~m}, 1 \mathrm{H}), 7.49-7.40(\mathrm{~m}, 2 \mathrm{H}), 7.38(\mathrm{~d}, J=12.5 \mathrm{~Hz}, 1 \mathrm{H}), 5.64(\mathrm{t}, J=6.7 \mathrm{~Hz}, 1 \mathrm{H}), 5.24(\mathrm{~d}, J$ $=12.5 \mathrm{~Hz}, 1 \mathrm{H}), 4.99-4.82(\mathrm{~m}, 2 \mathrm{H}), 3.37(\mathrm{~s}, 3 \mathrm{H}), 3.08-2.92(\mathrm{~m}, 2 \mathrm{H}), 1.65(\mathrm{~s}, 3 \mathrm{H}) .{ }^{13} \mathbf{C}$ NMR $(100$ $\left.\mathrm{MHz}, \mathrm{CDCl}_{3}\right) \delta 206.8,197.5,162.1,144.2,133.2,132.0,128.0,127.7,127.3,125.9,125.6,125.2,124.6$, 106.1, 99.9, 78.0, 57.1, 53.3, 41.3, 26.6. IR (atr, $\left.\mathrm{cm}^{-1}\right)$ 3056, 2969, 2934, 2840, 1954, 1677, 1619, 1591, 1505, 1454, 1436, 1339, 1309, 1247, 1200, 1232, 1096, 1045, 951, 855, 817, 748, 660, 477. HRMS (ESI+, M+Na) Calc'd for $\mathrm{C}_{20} \mathrm{H}_{20} \mathrm{O}_{2} \mathrm{Na} 315.1356$, found 315.1354. Enantiomeric purity was determined by SFC analysis in comparison with racemic material $\mathrm{T}_{\mathrm{R}}=4.5$ (minor) $\mathrm{T}_{\mathrm{R}}=5.3$ (major) (94:6 er shown); Chiracel AS-H column, $90 \% \mathrm{CO}_{2}$ to $10 \% \mathrm{MeOH}, 2.0$ $\left.\mathrm{mL} / \mathrm{min}, 220 \mathrm{~nm}, 135 \mathrm{bar}, 25^{\circ} \mathrm{C}\right)[\boldsymbol{\alpha}]_{D}^{24}=+75.3\left(\mathrm{c}=0.5, \mathrm{CHCl}_{3}\right)$. The absolute stereochemistry of this compound was assigned in analogy to $\mathbf{3 a}$.
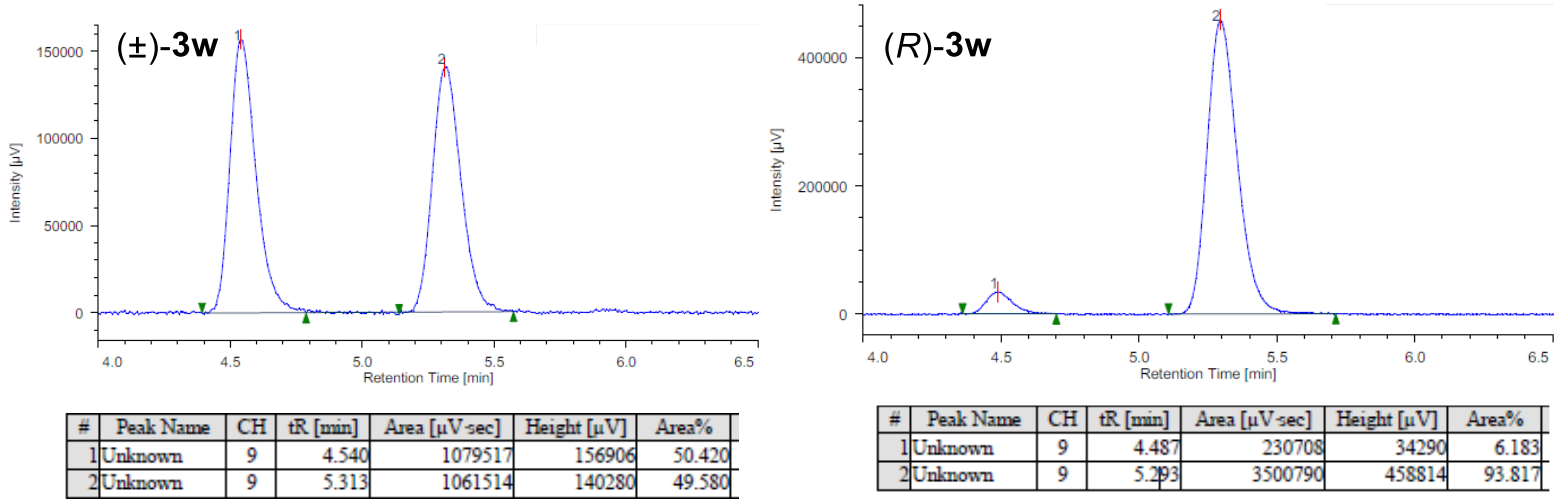


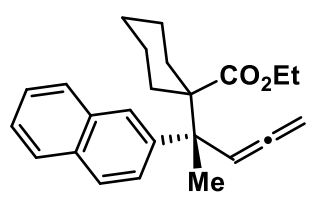

ethyl (R)-1-(2-(naphthalen-2-yl)penta-3,4-dien-2-yl)cyclohexane-1carboxylate $(\mathbf{3 x})$ - Was synthesized according to general procedure $2 \mathrm{a}$ using $1 \mathrm{a}(89.7 \mathrm{mg}, 0.4 \mathrm{mmol}),[\operatorname{Ir}(\operatorname{cod}) \mathrm{Cl}]_{2}(13.4 \mathrm{mg}, 20 \mu \mathrm{mol}, 5.0 \mathrm{~mol} \%)$, $(S)-\mathbf{L}_{1}$ (40.6 mg, $\left.80 \mu \mathrm{mol}, 20 \mathrm{~mol} \%\right), \mathbf{2 g}(251 \mu \mathrm{L}, 1.0 \mathrm{mmol}, 2.5$ equiv), and $\mathrm{La}(\mathrm{OTf})_{3}(17.6 \mathrm{mg}, 30 \mu \mathrm{mol}, 7.5 \mathrm{~mol} \%)$, and the reaction was run for 5 hours. The regiomeric ratio (rr) was determined to be $>20: 1$ by ${ }^{1} \mathrm{H}$ NMR analysis of the unpurified reaction mixture. The title compound was obtained by purification via $\mathrm{SiO}_{2}$ flash column chromatography using pentane:Et $\mathrm{t}_{2} \mathrm{O}(40: 1 \mathrm{v}: \mathrm{v})$ (NOTE: the ester from ketene acetal may co-elute with the product. In this case, the resulting clear oil was put in a high vac (5 bar) heating with $50{ }^{\circ} \mathrm{C}$ overnight.). The title compound was obtained as a clear slightly yellow oil which solidified to a white solid upon standing at $-20{ }^{\circ} \mathrm{C}$. $(114.4 \mathrm{mg}, 0.33 \mathrm{mmol}, 82 \%$ yield, $\left.\mathrm{MP}=42.1-42.7{ }^{\circ} \mathrm{C}\right) .{ }^{1} \mathbf{H}$ NMR $\left(400 \mathrm{MHz}, \mathrm{CDCl}_{3}\right) \delta 7.85-7.69(\mathrm{~m}, 4 \mathrm{H}), 7.51(\mathrm{dd}, J=8.8,2.1$ $\mathrm{Hz}, 1 \mathrm{H}), 7.48-7.41(\mathrm{~m}, 2 \mathrm{H}), 6.25(\mathrm{t}, J=6.7 \mathrm{~Hz}, 1 \mathrm{H}), 4.82-4.61(\mathrm{~m}, 2 \mathrm{H}), 3.95(\mathrm{qd}, J=7.2$, $1.2 \mathrm{~Hz}, 2 \mathrm{H}), 2.31-2.19(\mathrm{~m}, 2 \mathrm{H}), 1.73-1.46(\mathrm{~m}, 5 \mathrm{H}), 1.35-1.04(\mathrm{~m}, 5 \mathrm{H}), 0.99(\mathrm{t}, J=7.2 \mathrm{~Hz}$, $3 \mathrm{H}), 0.96-0.81(\mathrm{~m}, 1 \mathrm{H}){ }^{13} \mathbf{C}$ NMR $\left(100 \mathrm{MHz}, \mathrm{CDCl}_{3}\right) \delta 207.6,174.3,142.1,132.7,131.9$, $128.1,127.2$, 127.1, 127.0, 126.2, 125.7, 125.7, 96.7, 77.0, 60.0, 55.2, 47.0, 30.0, 30.0, 25.3, 24.0, 24.0, 21.9, 13.8. IR (atr, $\left.\mathrm{cm}^{-1}\right)$ 3056, 2978, 2936, 2862, 1954, 1713, 1598, 1506, 1451, 1385, 1347, 1303, 1204, 1130, 1095, 1026, 842, 816, 744, 477. HRMS (ESI+, M+Na) Calc'd for $\mathrm{C}_{24} \mathrm{H}_{28} \mathrm{O}_{2} \mathrm{Na} 371.1982$, found 371.1982. Enantiomeric purity was determined by HPLC analysis in comparison with racemic material $\mathrm{T}_{\mathrm{R}}=5.5$ (minor) $\mathrm{T}_{\mathrm{R}}=9.3$ (major) (95.5:4.5 er shown); Chiracel IC column, 98\% hexane to $2 \% \mathrm{iPrOH}, 1.0 \mathrm{~mL} / \mathrm{min}, 227 \mathrm{~nm}, 490 \mathrm{psi}, 26{ }^{\circ} \mathrm{C}$ ) $[\alpha]_{D}^{24}=-124.0\left(\mathrm{c}=0.5, \mathrm{CHCl}_{3}\right)$. The absolute stereochemistry of this compound was assigned in analogy to $\mathbf{3 a}$.
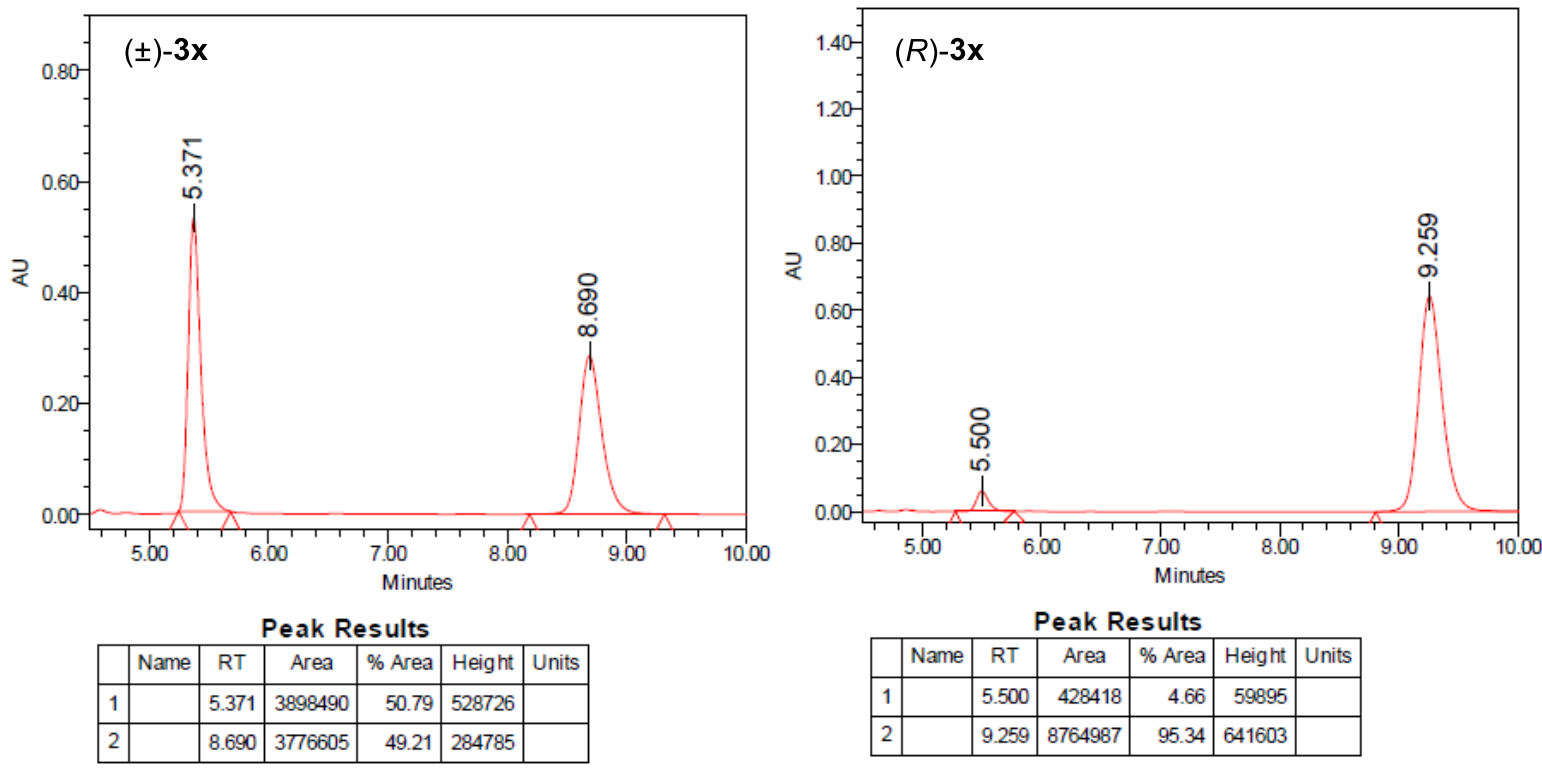


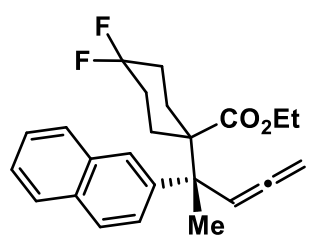

ethyl (R)-4,4-difluoro-1-(2-(naphthalen-2-yl)penta-3,4-dien-2yl)cyclohexane-1-carboxylate $(\mathbf{3 y})$ - Was synthesized according to general procedure $2 \mathrm{a}$ using $\mathbf{1 a}(89.7 \mathrm{mg}, 0.4 \mathrm{mmol}),[\mathrm{Ir}(\operatorname{cod}) \mathrm{Cl}]_{2}(13.4 \mathrm{mg}$, $20 \mu \mathrm{mol}, 5.0 \mathrm{~mol} \%),(S)-\mathbf{L}_{\mathbf{1}}(40.6 \mathrm{mg}, 80 \mu \mathrm{mol}, 20 \mathrm{~mol} \%), \mathbf{2 h}(257 \mu \mathrm{L}$, $1.0 \mathrm{mmol}, 2.5$ equiv), and $\mathrm{La}(\mathrm{OTf})_{3}(17.6 \mathrm{mg}, 30 \mu \mathrm{mol}, 7.5 \mathrm{~mol} \%)$, and the reaction was run for 14 hours. The regiomeric ratio (rr) was determined to be $>20: 1$ by ${ }^{1} \mathrm{H}$ NMR analysis of the unpurified reaction mixture. The title compound was obtained by purification via $\mathrm{SiO}_{2}$ flash column chromatography using pentane:Et ${ }_{2} \mathrm{O}$ (100:1 to 50:1 to 20:1 v:v) (NOTE: the ester from ketene acetal may co-elute with product. In this case, the resulting clear oil was put in a high vac (5 bar) heating with $50{ }^{\circ} \mathrm{C}$ overnight.). The title compound was obtained as a clear slightly yellow oil ( $88.0 \mathrm{mg}, 0.23 \mathrm{mmol}, 57 \%$ yield). ${ }^{1} \mathbf{H}$ NMR $\left(400 \mathrm{MHz}, \mathrm{CDCl}_{3}\right) \delta 7.85-7.74(\mathrm{~m}, 4 \mathrm{H}), 7.51-7.43(\mathrm{~m}, 3 \mathrm{H}), 6.22(\mathrm{t}, J=6.7 \mathrm{~Hz}, 1 \mathrm{H})$, $4.87-4.73(\mathrm{~m}, 2 \mathrm{H}), 3.95(\mathrm{q}, J=7.1 \mathrm{~Hz}, 1 \mathrm{H}), 2.38-2.28(\mathrm{~m}, 2 \mathrm{H}), 2.07-1.96(\mathrm{~m}, 2 \mathrm{H}), 1.79-$ $1.53(\mathrm{~m}, 7 \mathrm{H}), 0.95(\mathrm{t}, J=7.1 \mathrm{~Hz}, 3 \mathrm{H}) .{ }^{13} \mathrm{C}$ NMR $\left(100 \mathrm{MHz}, \mathrm{CDCl}_{3}\right) \delta 207.7,173.2,141.3$, $132.7,132.0,128.1,127.2,126.9,126.6,126.3,126.0,125.9,122.95(\mathrm{dd}, J=242.3,238.9 \mathrm{~Hz})$, 96.0, 77.5, 60.7, 54.1, 46.5, 31.63 (ddd, $J=25.2,22.8,2.1 \mathrm{~Hz}, 2 \mathrm{C}), 22.1,26.45(\mathrm{dd}, J=17.6$, $9.8 \mathrm{~Hz}, 2 \mathrm{C}), 13.7 .{ }^{19} \mathbf{F}$ NMR $\left(376 \mathrm{MHz}, \mathrm{CDCl}_{3}\right) \delta-92.08(\mathrm{~d}, J=234.1 \mathrm{~Hz}),-103.35(\mathrm{~d}, J=$ $234.2 \mathrm{~Hz}$ ). IR $\left(\mathrm{atr}, \mathrm{cm}^{-1}\right)$ 3062, 2983, 2949, 1954, 1716, 1443, 1377, 1305, 1234, 1207, 1127 , 1112, 1092, 1024, 982, 860, 817, 744, 476. HRMS (ESI+, M+Na) Calc'd for $\mathrm{C}_{24} \mathrm{H}_{26} \mathrm{~F}_{2} \mathrm{O}_{2} \mathrm{Na}$ 407.1793, found 407.1801. Enantiomeric purity was determined by HPLC analysis in comparison with racemic material $\mathrm{T}_{\mathrm{R}}=5.5$ (minor) $\mathrm{T}_{\mathrm{R}}=6.0$ (major) (98:2 er shown); Chiracel IC column, $99 \%$ hexane to $\left.1 \% \mathrm{iPrOH}, 1.0 \mathrm{~mL} / \mathrm{min}, 215 \mathrm{~nm}, 478 \mathrm{psi}, 27^{\circ} \mathrm{C}\right)[\boldsymbol{\alpha}]_{\boldsymbol{D}}^{\mathbf{2 5}}=-101.6(\mathrm{c}$ $\left.=0.5, \mathrm{CHCl}_{3}\right)$. The absolute stereochemistry of this compound was assigned in analogy to $\mathbf{3 a}$.
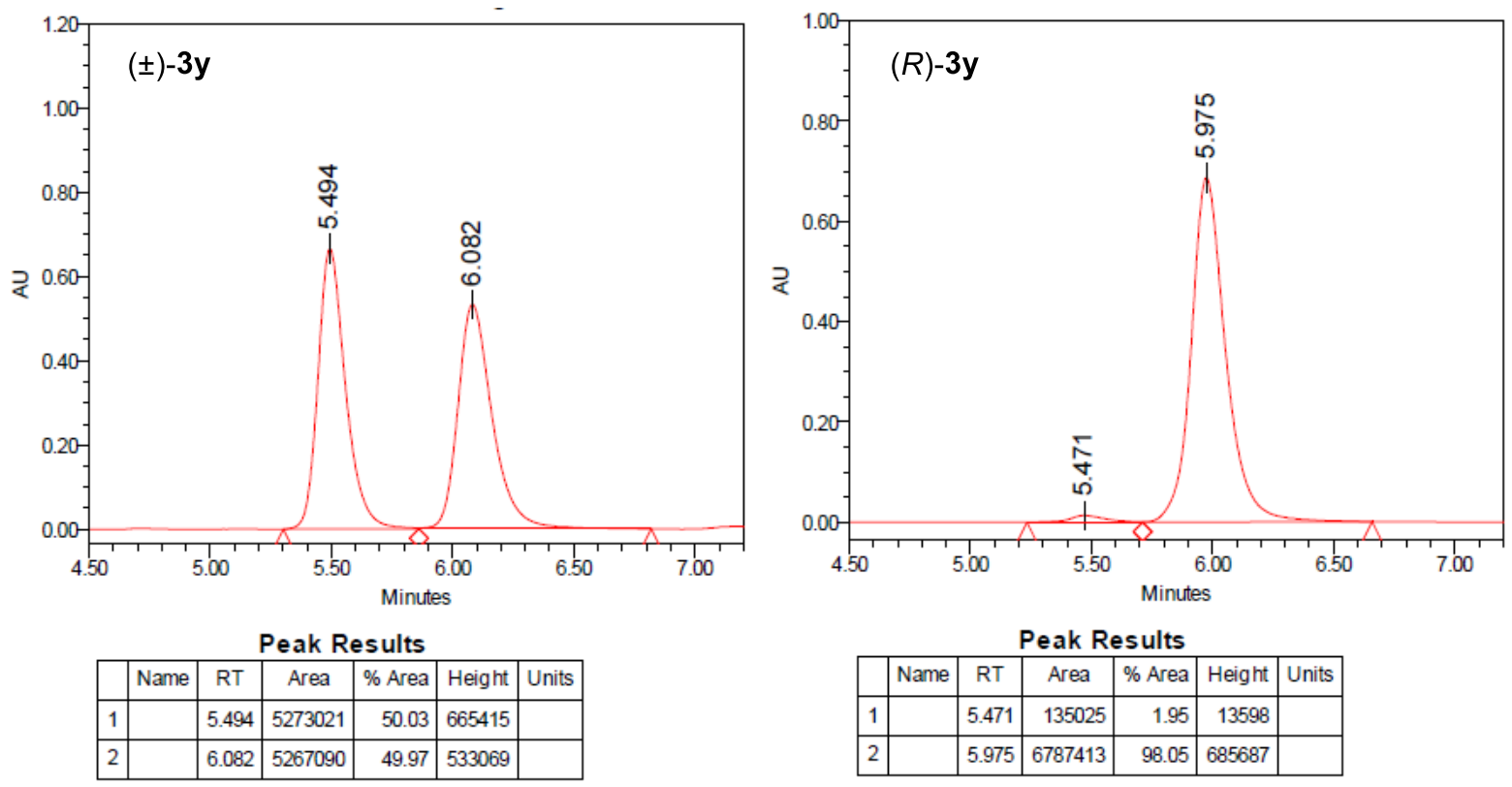
<smiles>C=CC[C@](C)(c1ccc2ccccc2c1)C(CCO)(CCO)C(=O)OCC</smiles>

ethyl (R)-4-(2-(naphthalen-2-yl)penta-3,4-dien-2-yl)tetrahydro-2Hpyran-4-carboxylate $(\mathbf{3 z})$ - Was synthesized according to general procedure 2a using 1a $(89.7 \mathrm{mg}, 0.4 \mathrm{mmol}),[\operatorname{Ir}(\operatorname{cod}) \mathrm{Cl}]_{2}(13.4 \mathrm{mg}, 20$ $\mu \mathrm{mol}, 5.0 \mathrm{~mol} \%),(S)-\mathbf{L}_{1}(40.6 \mathrm{mg}, 80 \mu \mathrm{mol}, 20 \mathrm{~mol} \%), \mathbf{2 i}(235 \mu \mathrm{L}, 1.0$ mmol, 2.5 equiv), and $\mathrm{La}(\mathrm{OTf})_{3}(17.6 \mathrm{mg}, 30 \mu \mathrm{mol}, 7.5 \mathrm{~mol} \%)$, and the reaction was run for 14 hours. The regiomeric ratio (rr) was determined to be $>20: 1$ by ${ }^{1} \mathrm{H}$ NMR analysis of the unpurified reaction mixture. The title compound was obtained by purification via $\mathrm{SiO}_{2}$ flash column chromatography using pentane:Et $\mathrm{t}_{2} \mathrm{O}(10: 1$ to 5:1 v:v) (NOTE: the ester from ketene acetal may co-elute with product. In this case, the resulting clear oil was put in a high vac (5 bar) heating with $50{ }^{\circ} \mathrm{C}$ overnight.). The title compound was obtained as a clear slightly yellow oil $\left(95.8 \mathrm{mg}, 0.27 \mathrm{mmol}, 68 \%\right.$ yield). ${ }^{1} \mathbf{H} \mathbf{~ N M R}\left(400 \mathrm{MHz}, \mathrm{CDCl}_{3}\right) \delta 7.84-$ $7.72(\mathrm{~m}, 4 \mathrm{H}), 7.53-7.41(\mathrm{~m}, 3 \mathrm{H}), 6.20(\mathrm{t}, J=6.7 \mathrm{~Hz}, 1 \mathrm{H}), 4.84-4.71(\mathrm{~m}, 2 \mathrm{H}), 4.00-3.83$ $(\mathrm{m}, 4 \mathrm{H}), 3.25(\mathrm{tdd}, J=12.1,7.5,1.8 \mathrm{~Hz}, 2 \mathrm{H}), 2.17-2.09(\mathrm{~m}, 2 \mathrm{H}), 1.76(\mathrm{tt}, J=12.7,5.0 \mathrm{~Hz}$, $2 \mathrm{H}), 1.66(\mathrm{~s}, 3 \mathrm{H}), 0.93(\mathrm{t}, J=7.1 \mathrm{~Hz}, 3 \mathrm{H}) .{ }^{13} \mathbf{C} \mathbf{N M R}\left(100 \mathrm{MHz}, \mathrm{CDCl}_{3}\right) \delta 207.8,173.5,141.2$, $132.7,132.0,128.1,127.2,126.9,126.6,126.5,125.9,125.9,95.8,77.3,66.1,66.0,60.5,53.5$, 46.6, 30.2, 30.1, 21.5, 13.7. IR (atr, $\left.\mathrm{cm}^{-1}\right)$ 3056, 2977, 2854, 1954, 1715, 1598, 1505, 1471, $1441,1389,1365,1350,1300,1276,1252,1223,1200,1135,1105,1095,1050,1029,944$, 861, 818, 745, 677, 549, 477, 420. HRMS (ESI+, M+Na) Calc'd for $\mathrm{C}_{23} \mathrm{H}_{26} \mathrm{O}_{3} \mathrm{Na} 373.1774$, found 373.1779. Enantiomeric purity was determined by HPLC analysis in comparison with racemic material $\mathrm{T}_{\mathrm{R}}=25.2$ (minor) $\mathrm{T}_{\mathrm{R}}=27.8$ (major) (96:4 er shown); Chiracel IC column, $99 \%$ hexane to $\left.1 \% \mathrm{iPrOH}, 1.0 \mathrm{~mL} / \mathrm{min}, 224 \mathrm{~nm}, 483 \mathrm{psi}, 27{ }^{\circ} \mathrm{C}\right)[\boldsymbol{\alpha}]_{D}^{25}=-133.2(\mathrm{c}=0.5$, $\mathrm{CHCl}_{3}$ ). The absolute stereochemistry of this compound was assigned in analogy to 3a.
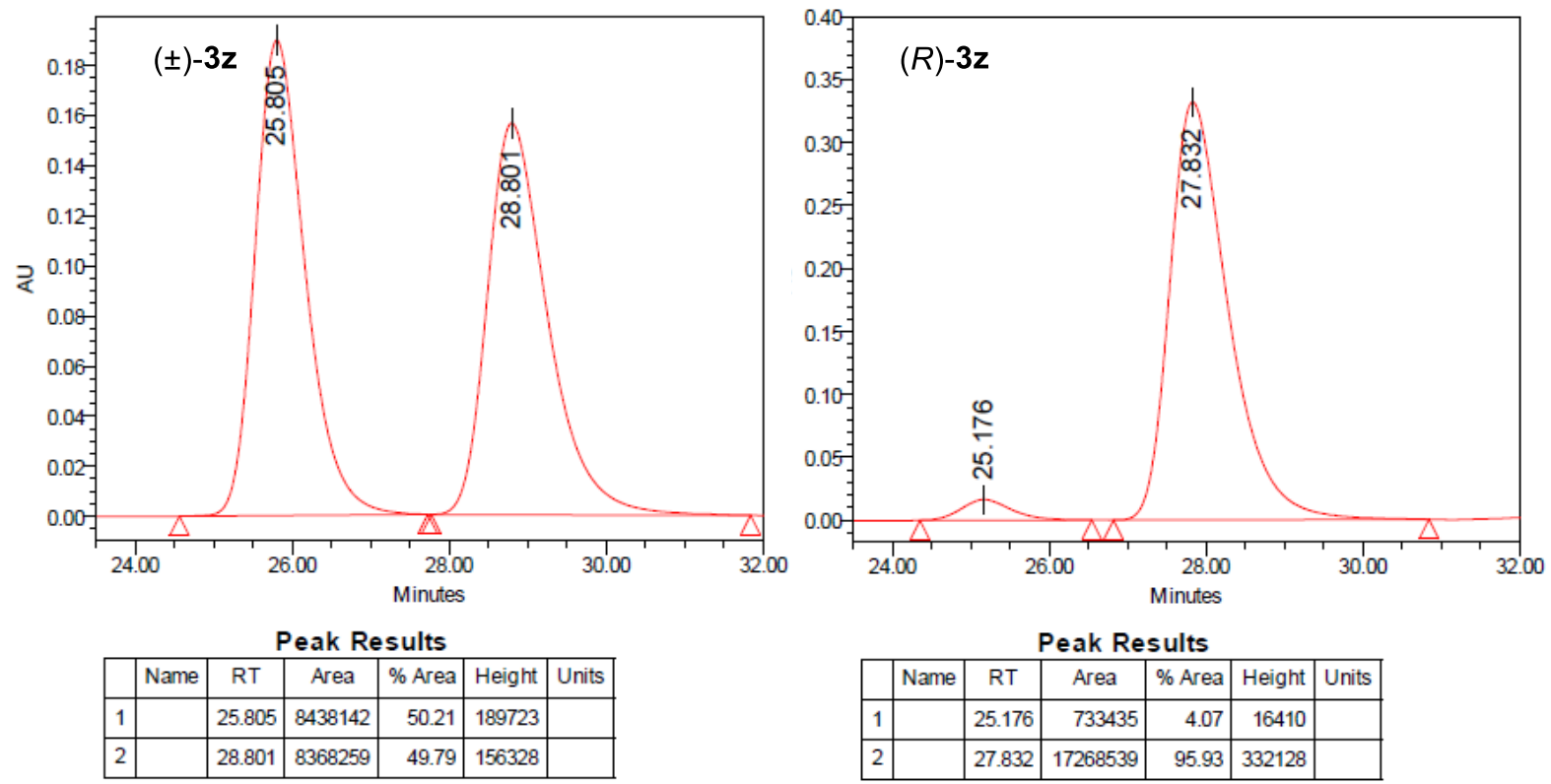


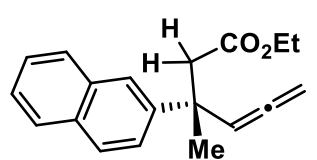

ethyl (R)-3-methyl-3-(naphthalen-2-yl)hexa-4,5-dienoate (3aa) - A dry $5 \mathrm{~mL}$ vial containing a magnetic stir-bar was charged with $( \pm)-1(0.2$ mmol, 1 equiv), $[\operatorname{Ir}(\operatorname{cod}) \mathrm{Cl}]_{2}(6.72 \mathrm{mg}, 10 \mu \mathrm{mol}, 5.0 \mathrm{~mol} \%)$ and $(S)-\mathbf{L} \mathbf{1}$ (20.3 mg, $40 \mu \mathrm{mol}, 20 \mathrm{~mol} \%)$. These solids were taken up in wet THF (2.0 $\mathrm{mL},[( \pm)-1]=0.1 \mathrm{M}$, containing $25 \mathrm{~mol} \%(0.5 \mathrm{mmol})$ of $\left.\mathrm{H}_{2} \mathrm{O}\right)$ and the resulting solution was stirred under dry Ar gas for 10 minutes. tert-butyl((2-ethoxyallyl)oxy)dimethylsilane (2k) (70.6 $\mu \mathrm{L}, 0.3 \mathrm{mmol}, 1.5$ equiv) and $\mathrm{Zn}(\mathrm{OTf})_{2}(5.45 \mathrm{mg}, 15 \mu \mathrm{mol}, 7.5 \mathrm{~mol} \%)$ were then added, and the vial was sealed with a rubber septum. The reaction was stirred at $45^{\circ} \mathrm{C}$. After 10 minutes, $2 \mathbf{k}(40.0 \mu \mathrm{L}, 0.17 \mathrm{mmol}, 0.85$ equiv) was added maintaining the temperature, the reaction was stirred for 10 minutes, and another $2 \mathbf{k}(40.0 \mu \mathrm{L}, 0.17 \mathrm{mmol}, 0.85$ equiv) was added. After 10 minutes, $2 \mathbf{k}$ ( $37.6 \mu \mathrm{L}, 0.16 \mathrm{mmol}, 0.80$ equiv) was added, the vial was re-purged with Ar and then sealed with a PTFE screw cap. The reaction was stirred for 5.5 hours, and then the mixture was passed through a plug of Celite eluting with $\mathrm{Et}_{2} \mathrm{O}$ and the filtrate was concentrated in vacuo. The regiomeric ratio (rr) was determined to be $>20: 1$ by ${ }^{1} \mathrm{H}$ NMR analysis of the unpurified reaction mixture. The title compound was obtained by purification via $\mathrm{SiO}_{2}$ flash column chromatography using pentane: $\mathrm{Et}_{2} \mathrm{O}(50: 1 \mathrm{v}: \mathrm{v}$ The title compound was obtained as a clear slightly yellow oil $(39.7 \mathrm{mg}, 0.14 \mathrm{mmol}, 71 \%$ yield $) .{ }^{1} \mathbf{H}$ NMR $\left(400 \mathrm{MHz}, \mathrm{CDCl}_{3}\right) \delta 7.85-$ $7.74(\mathrm{~m}, 4 \mathrm{H}), 7.59-7.50(\mathrm{~m}, 1 \mathrm{H}), 7.50-7.38(\mathrm{~m}, 2 \mathrm{H}), 5.64(\mathrm{t}, J=6.7 \mathrm{~Hz}, 1 \mathrm{H}), 4.91(\mathrm{dd}, J=$ 6.7, $1.1 \mathrm{~Hz}, 2 \mathrm{H}), 4.01-3.92(\mathrm{~m}, 2 \mathrm{H}), 2.95-2.81(\mathrm{~m}, 2 \mathrm{H}), 1.67(\mathrm{~s}, 3 \mathrm{H}), 1.04(\mathrm{t}, J=7.1 \mathrm{~Hz}$, 3H). ${ }^{13} \mathrm{C}$ NMR $\left(100 \mathrm{MHz}, \mathrm{CDCl}_{3}\right) \delta$ 206.9, 171.1, 143.9, 133.2, 132.0, 128.0, 127.7, 127.4, 125.9, 125.6, 125.1, 124.4, 99.4, 78.0, 60.0, 46.3, 40.9, 26.8, 14.0. IR (atr, $\left.\mathrm{cm}^{-1}\right)$ 3057, 2962, 2924, 2853, 1955, 1730, 1632, 1599, 1505, 1462, 1367, 1338, 1302, 1273, 1217, 1197, 1128, 1030, 948, 851, 815, 745, 661, 475. HRMS (ESI+, M+H) Calc'd for $\mathrm{C}_{19} \mathrm{H}_{21} \mathrm{O}_{2} 281.1536$, found 281.1535. Enantiomeric purity was determined by SFC analysis in comparison with racemic material $\mathrm{T}_{\mathrm{R}}=7.7$ (minor) $\mathrm{T}_{\mathrm{R}}=9.0$ (major) (96:4 er shown); Chiralcel OJ-H column, $90 \% \mathrm{CO}_{2}$ to $\left.10 \% \mathrm{MeOH}, 2.0 \mathrm{~mL} / \mathrm{min}, 220 \mathrm{~nm}, 125 \mathrm{bar}, 25{ }^{\circ} \mathrm{C}\right)[\alpha]_{D}^{27}=+12.0\left(\mathrm{c}=0.48, \mathrm{CHCl}_{3}\right)$. The absolute stereochemistry of this compound was assigned in analogy to $\mathbf{3 a}$.
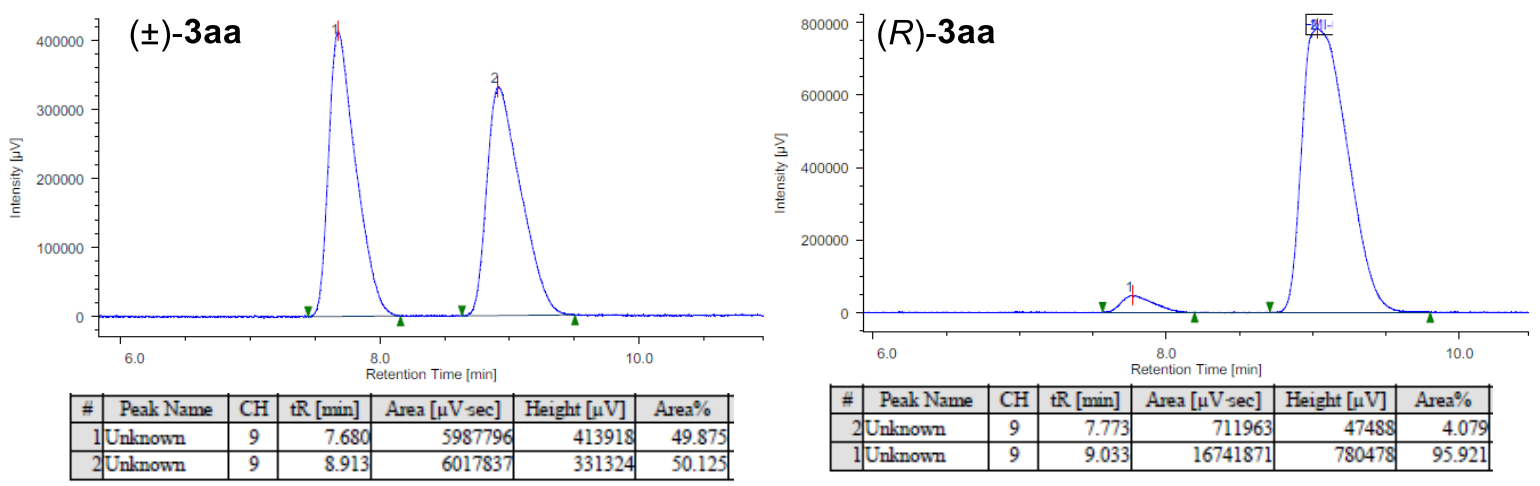


\section{Derivatization of the Products}

Molybdenium-catalyzed Hydrosilylation ${ }^{17}$

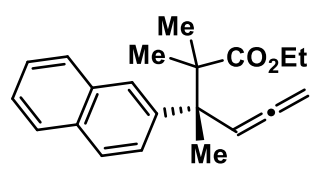

$(R)-3 a$

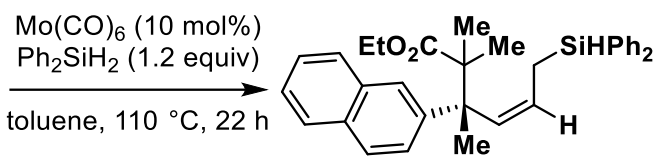

$(R, Z)-5$

$>20: 1 \mathrm{rr} ;>20: 1 \mathrm{Z:E}$

ethyl $(R, Z)-6$-(diphenylsilyl)-2,2,3-trimethyl-3-(naphthalen-2-yl)hex-4-enoate $[(R, Z)-5]-\mathrm{A}$ dried $1 \mathrm{~mL}$ vial containing a magnetic stir-bar was charged with $(R)-3 \mathbf{a}(61.7 \mathrm{mg}, 0.20 \mathrm{mmol}$, 1 equiv), $\mathrm{Mo}(\mathrm{CO})_{6}(5.3 \mathrm{mg}, 20 \mu \mathrm{mol}, 10 \mathrm{~mol} \%)$ and diphenylsilane $(44.7 \mu \mathrm{L}, 0.24 \mathrm{mmol}, 1.2$ equiv), which were taken up with toluene $(0.2 \mathrm{~mL})$. The vial was purged with dried Ar and then sealed with a screw cap. The reaction was stirred at $110{ }^{\circ} \mathrm{C}$ for 22 hours. The regiomeric ratio (rr) and the $Z: E$ ratio were determined to be $>20: 1$ by ${ }^{1} \mathrm{H}$ NMR analysis of the unpurified reaction mixture. The solution was passed through a plug of Celite eluting with $\mathrm{Et}_{2} \mathrm{O}$ and the filtrate was concentrated in vacuo. The crude residue was purified via $\mathrm{SiO}_{2}$ flash column chromatography using pentane: $\mathrm{Et}_{2} \mathrm{O}(15: 1 \mathrm{v}: \mathrm{v})$, and the title compound was isolated as a clear colorless oil (71.5 mg, $0.15 \mathrm{mmol}, 73 \%$ yield). ${ }^{1} \mathbf{H}$ NMR $\left(400 \mathrm{MHz}, \mathrm{CDCl}_{3}\right) \delta 7.87-7.78(\mathrm{~m}$, $3 \mathrm{H}), 7.71(\mathrm{~d}, J=8.7 \mathrm{~Hz}, 1 \mathrm{H}), 7.56-7.52(\mathrm{~m}, 1 \mathrm{H}), 7.51-7.45(\mathrm{~m}, 2 \mathrm{H}), 7.42-7.34(\mathrm{~m}, 3 \mathrm{H})$, $7.34-7.26(\mathrm{~m}, 3 \mathrm{H}), 7.18-7.07(\mathrm{~m}, 4 \mathrm{H}), 6.41(\mathrm{dt}, J=11.6,1.7 \mathrm{~Hz}, 1 \mathrm{H}), 5.57(\mathrm{dt}, J=11.6,8.6$ $\mathrm{Hz}, 1 \mathrm{H}), 4.70-4.64(\mathrm{~m}, 1 \mathrm{H}), 4.09-3.94(\mathrm{~m}, 2 \mathrm{H}), 1.66(\mathrm{~s}, 3 \mathrm{H}), 1.56-1.36(\mathrm{~m}, 2 \mathrm{H}), 1.21$ (s, $3 \mathrm{H}), 1.12-1.05(\mathrm{~m}, 6 \mathrm{H}) .{ }^{13} \mathrm{C}$ NMR $\left(100 \mathrm{MHz}, \mathrm{CDCl}_{3}\right) \delta 176.7,142.1,135.2(2 \mathrm{C}), 135.0(2 \mathrm{C})$, 134.8, 133.6, 133.5, 132.9, 132.0, 129.6, 129.4, 128.2 (2C), 127.9 (2C), 127.7 (2C), 127.4, 127.3, 126.5, 126.2, 125.7, 125.5, 60.3, 49.6, 48.5, 22.9, 22.3, 21.9, 16.0, 14.0. IR (atr, cm $\left.{ }^{-1}\right) 3051$, 2978, 2126, 1716, 1630, 1598, 1504, 1465, 1428, 1264, 1138, 1127, 1115, 1081, 1026, 996, 857, 807, 730, 699, 479. HRMS (ESI+, M+Na) Calc'd for $\mathrm{C}_{33} \mathrm{H}_{36} \mathrm{SiO}_{2} \mathrm{Na}$ 515. 2377, found 515.2378. Enantiomeric purity was determined by HPLC analysis in comparison with racemic material $\mathrm{T}_{\mathrm{R}}=7.9$ (minor) $\mathrm{T}_{\mathrm{R}}=9.7$ (major) (99.5:0.5 er shown); Chiracel IC column, $99.5 \%$ hexane to $\left.0.5 \% \mathrm{iPrOH}, 1.0 \mathrm{~mL} / \mathrm{min}, 236 \mathrm{~nm}, 480 \mathrm{psi}, 26^{\circ} \mathrm{C}\right)[\alpha]_{D}^{24}=-82.9\left(\mathrm{c}=0.5, \mathrm{CHCl}_{3}\right)$.
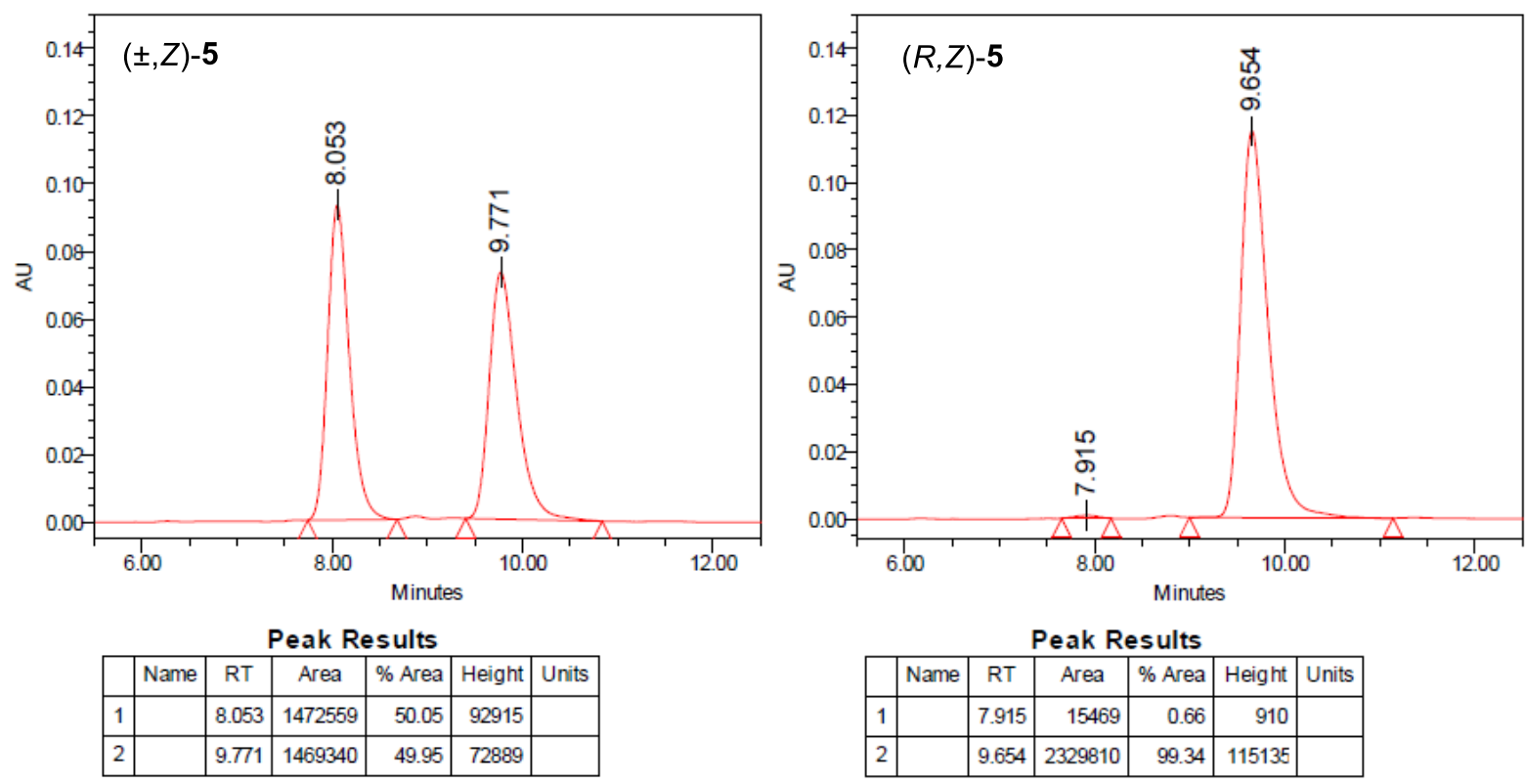

Palladium catalyzed aminoarylation ${ }^{18}$ 


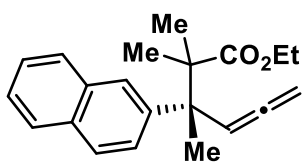

$(R)-\mathbf{3 a}$

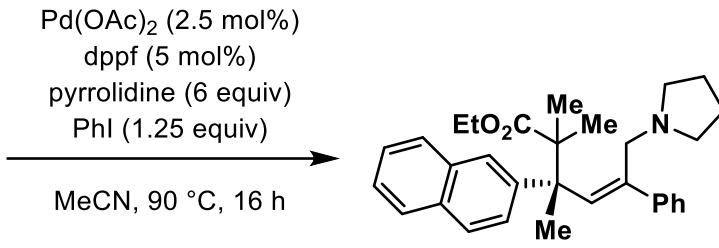

$(R, Z)-6$

$>20: 1 \mathrm{rr} ;>20: 1 Z: E$

ethyl (R,Z)-2,2,3-trimethyl-3-(naphthalen-2-yl)-5-phenyl-6-(pyrrolidin-1-yl)hex-4-enoate [(S,Z)-6] - A dried Schlenk tube containing a magnetic stir bar was added $\operatorname{Pd}(\mathrm{OAc})_{2}(1.12 \mathrm{mg}$, $5.0 \mu \mathrm{mol}, 2.5 \mathrm{~mol} \%)$, dppe $(3.98 \mathrm{mg}, 10.0 \mu \mathrm{mol}, 5 \mathrm{~mol} \%)$, iodobenzene $(28 \mu \mathrm{L}, 0.25 \mathrm{mmol}$, 1.25 equiv) and pyrrolidine ( $0.1 \mathrm{~mL}, 1.2 \mathrm{mmol}, 6.0$ equiv), which were taken up with acetonitrile $(0.5 \mathrm{~mL})$. The mixture was heated to $90^{\circ} \mathrm{C}$, a solution of $(R)-3 \mathbf{a}(61.7 \mathrm{mg}, 0.2 \mathrm{mmol}$, 1 equiv) in acetonitrile $(0.5 \mathrm{~mL})$ was added via syringe. The mixture was refluxed for 16 hours after which time it was cooled to room temperature. The reaction mixture was then passed through a plug of Celite eluting with $\mathrm{Et}_{2} \mathrm{O}$, and filtrate was concentrated in vacuo. The regiomeric ratio (rr) and the $Z: E$ ratio were determined to be $>20: 1$ by ${ }^{1} \mathrm{H} N M R$ analysis of the unpurified reaction mixture. The crude residue was purified by $\mathrm{SiO}_{2}$ flash column silica gel chromatography using hexane:EtOAc (3:1 to $1: 4 \mathrm{v}: \mathrm{v})$. The title compound was obtained as a slightly yellow oil $\left(63.5 \mathrm{mg}, 0.14 \mathrm{mmol}, 70 \%\right.$ yield). ${ }^{1} \mathbf{H} \mathbf{~ N M R}\left(400 \mathrm{MHz}, \mathrm{CDCl}_{3}\right) \delta 7.87-$ $7.68(\mathrm{~m}, 4 \mathrm{H}), 7.53-7.38(\mathrm{~m}, 5 \mathrm{H}), 7.35-7.30(\mathrm{~m}, 2 \mathrm{H}), 7.28-7.20(\mathrm{~m}, 1 \mathrm{H}), 6.78(\mathrm{~s}, 1 \mathrm{H}), 4.10$ $-3.91(\mathrm{~m}, 2 \mathrm{H}), 2.93-2.77(\mathrm{~m}, 2 \mathrm{H}), 2.05-1.90(\mathrm{~m}, 4 \mathrm{H}), 1.87(\mathrm{~s}, 3 \mathrm{H}), 1.41-1.30(\mathrm{~m}, 4 \mathrm{H})$, $1.22(\mathrm{~s}, 3 \mathrm{H}), 1.13-1.03(\mathrm{~m}, 6 \mathrm{H}) .{ }^{13} \mathrm{C}$ NMR $\left(100 \mathrm{MHz}, \mathrm{CDCl}_{3}\right) \delta 176.6,144.2,142.4,140.5$, 137.4, 132.8, 131.9, 128.1, 127.8, 127.7 (2C), 127.3, 127.2, 127.0 (2C), 126.4, 126.3, 125.7, 125.5, 60.4, 54.6, 53.7 (2C), 50.2, 48.8, 23.3 (2C), 23.2, 22.4, 22.1, 13.9. IR (atr, $\mathrm{cm}^{-1}$ ) 3053, 2975, 2782, 1716, 1598, 1462, 1379, 1264, 1142, 1088, 1024, 858, 810, 743, 699, 479. HRMS (ESI+, M+H) Calc'd for $\mathrm{C}_{31} \mathrm{H}_{38} \mathrm{NO}_{2}$ 456.2897, found 456.2899. Enantiomeric purity was determined by HPLC analysis in comparison with racemic material $\mathrm{T}_{\mathrm{R}}=5.7$ (major) $\mathrm{T}_{\mathrm{R}}=6.6$ (minor) (99.5:0.5 er shown); Chiracel IC column, 50\% hexane to 50\% $\mathrm{iPrOH}, 1.0 \mathrm{~mL} / \mathrm{min}, 225$ $\left.\mathrm{nm}, 1120 \mathrm{psi}, 25^{\circ} \mathrm{C}\right)[\boldsymbol{\alpha}]_{\boldsymbol{D}}^{23}=+28.0\left(\mathrm{c}=0.5, \mathrm{CHCl}_{3}\right)$.
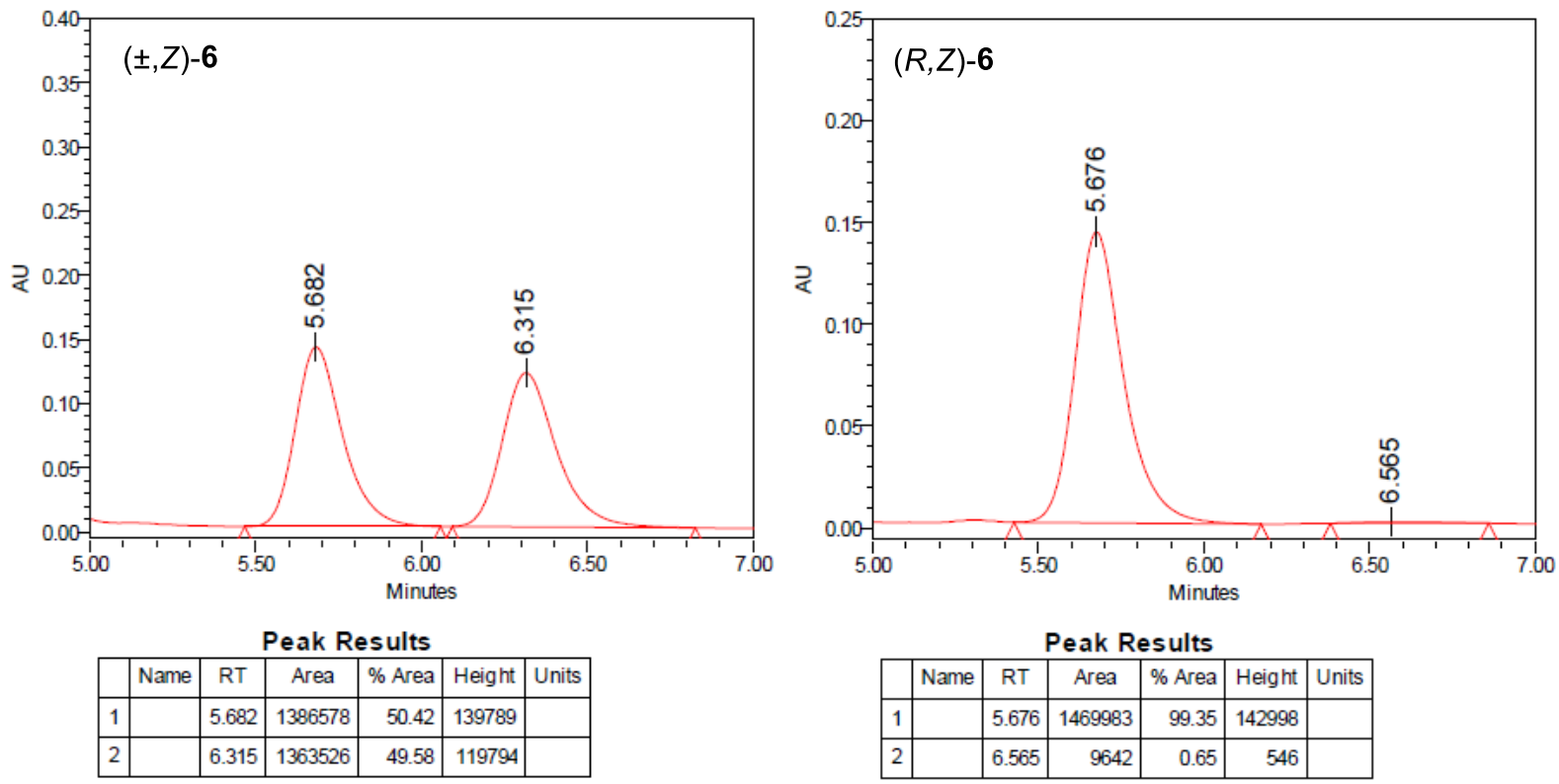
Conversion of the ester motif to a carboxylic acid:
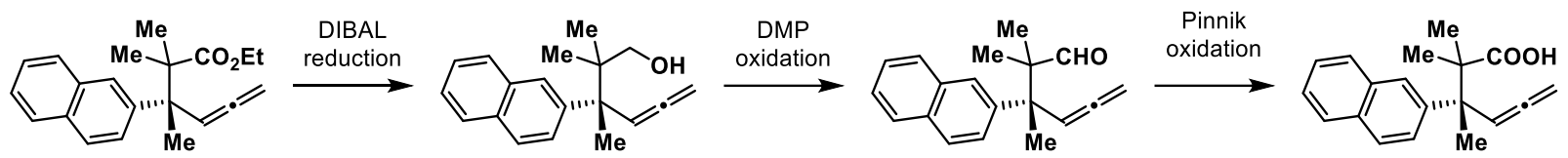

NOTE: this reaction sequence can be conducted without any $\mathrm{SiO}_{2}$ flash column chromatographic purification.

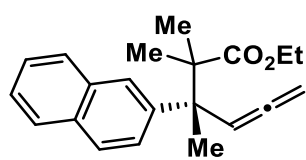

$(R)-3 a$

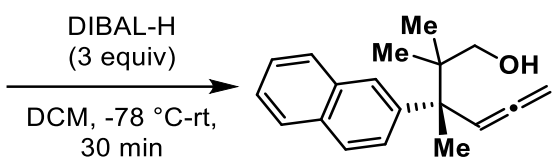

$(R)-\mathbf{S 3}$

(R)-2,2,3-trimethyl-3-(naphthalen-2-yl)hexa-4,5-dien-1-ol $[(R)-S 3]$ - A dried $50 \mathrm{~mL}$ flask containing magnetic stir bar was charged with 3a (700 mg, $2.27 \mathrm{mmol}, 1$ equiv) which was taken up with DCM $(11.3 \mathrm{~mL})$. The mixture was cooled to $-78^{\circ} \mathrm{C}$ and DIBAL-H $(6.81 \mathrm{~mL}$, $6.81 \mathrm{mmol}, 1 \mathrm{M}$ in toluene, 3 equiv) was added drop-wise over 3 minutes. The resulting mixture was warmed to room temperature and stirred for 30 minutes. The reaction was cooled to $0{ }^{\circ} \mathrm{C}$ and quenched with saturated Rochelle's Salt. The resulting biphasic mixture was stirred vigorously for 1 hour. The organics were extracted with DCM $(3 \times 30 \mathrm{~mL})$ and the combined organic layers were washed with brine, dried over $\mathrm{Na}_{2} \mathrm{SO}_{4}$, filtered and concentrated in vacuo. The crude residue was purified via $\mathrm{SiO}_{2}$ flash column chromatography using pentane:Et $\mathrm{t}_{2} \mathrm{O}(4: 1$ $\mathrm{v}: \mathrm{v})$. The title compound was isolated as a white solid $(578 \mathrm{mg}, 2.17 \mathrm{mmol}, 96 \%$ yield, MP = 88.9-89.0 $\left.{ }^{\circ} \mathrm{C}\right) .{ }^{1} \mathbf{H}$ NMR $\left(400 \mathrm{MHz}, \mathrm{CDCl}_{3}\right) \delta 7.88-7.75(\mathrm{~m}, 4 \mathrm{H}), 7.65-7.57(\mathrm{~m}, 1 \mathrm{H}), 7.52-$ $7.42(\mathrm{~m}, 2 \mathrm{H}), 6.21(\mathrm{t}, J=6.6 \mathrm{~Hz}, 1 \mathrm{H}), 4.86-4.70(\mathrm{~m}, 2 \mathrm{H}), 3.51(\mathrm{q}, J=11.1 \mathrm{~Hz}, 2 \mathrm{H}), 1.64(\mathrm{~s}$, $3 \mathrm{H}), 1.36(\mathrm{br}, 1 \mathrm{H}), 0.99(\mathrm{~d}, J=4.2 \mathrm{~Hz}, 6 \mathrm{H}) .{ }^{13} \mathbf{C ~ N M R}\left(100 \mathrm{MHz}, \mathrm{CDCl}_{3}\right) \delta 207.5,142.8,132.7$, 131.7, 128.1, 127.2, 127.0 (2C), 126.5, 125.8, 125.7, 97.3, 76.9, 69.7, 46.3, 41.8, 21.8, 21.1, 20.9. IR (atr, $\left.\mathrm{cm}^{-1}\right) 3595,3387,3056,2976,1952,1690,1690,1630,1597,1505,1461,1377$, 1276, 1126, 1026, 948, 843, 818, 762, 743, 476. HRMS (ESI+, M+Na) Calc'd for $\mathrm{C}_{19} \mathrm{H}_{22} \mathrm{ONa}$ 289.1563, found 289.1563. Enantiomeric purity was determined by HPLC analysis in comparison with racemic material $\mathrm{T}_{\mathrm{R}}=16.3$ (minor) $\mathrm{T}_{\mathrm{R}}=24.1$ (major) (99:1 er shown); Chiracel OJ-H column, $90 \%$ hexane to $\left.10 \% \mathrm{iPrOH}, 1.0 \mathrm{~mL} / \mathrm{min}, 218 \mathrm{~nm}, 600 \mathrm{psi}, 25^{\circ} \mathrm{C}\right)[\boldsymbol{\alpha}]_{\boldsymbol{D}}^{\mathbf{2 4}}$ $=-207.2\left(\mathrm{c}=0.5, \mathrm{CHCl}_{3}\right)$.
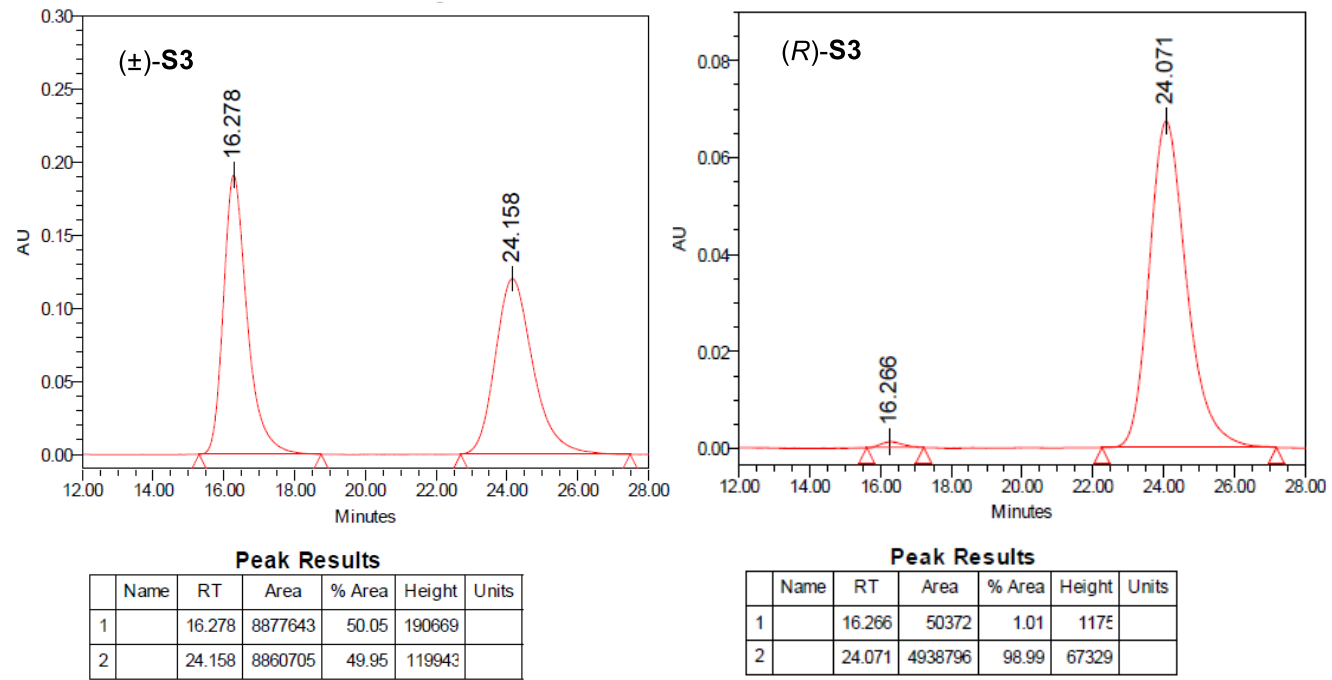

Peak Results
\begin{tabular}{|l|c|c|r|r|r|r|}
\hline & Name & RT & Area & $\%$ Area & Height & Units \\
\hline 1 & & 16.266 & 50372 & 1.01 & 1175 & \\
\hline 2 & & 24.071 & 4938796 & 98.99 & 67329 & \\
\hline
\end{tabular}




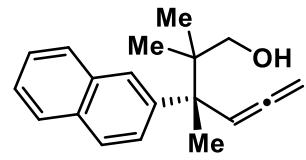

$(R)-\mathbf{S 3}$

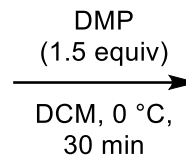

$30 \mathrm{~min}$

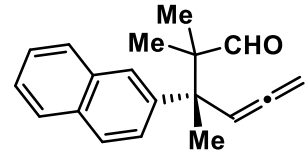

$(R)-\mathbf{S 4}$

(R)-2,2,3-trimethyl-3-(naphthalen-2-yl)hexa-4,5-dienal [(R)-S4] - A dried $25 \mathrm{~mL}$ flask containing magnetic stir bar was charged with $\mathbf{S 3}$ (450 mg, $1.69 \mathrm{mmol}, 1$ equiv) which was taken up with DCM $(8.5 \mathrm{~mL})$. The solution was cooled to $0{ }^{\circ} \mathrm{C}$ and DMP $(1.08 \mathrm{~g}, 2.53 \mathrm{mmol}$, 1.5 equiv) was added. The reaction was stirred for 30 minutes maintaining the temperature. The reaction was diluted $\mathrm{Et}_{2} \mathrm{O}(15 \mathrm{~mL})$, filtered through a plug of $\mathrm{SiO}_{2}$ eluting with $\mathrm{Et}_{2} \mathrm{O}(30 \mathrm{~mL})$, and the filtrate was concentrated in vacuo. The crude residue was purified via $\mathrm{SiO}_{2}$ flash column chromatography using pentane: $\mathrm{Et}_{2} \mathrm{O}(20: 1 \mathrm{v}: \mathrm{v})$, and the title compound was isolated as a white solid (400 mg, $1.51 \mathrm{mmol}, 90 \%$ yield, $\left.\mathrm{MP}=63.3-63.5^{\circ} \mathrm{C}\right){ }^{\mathbf{1}} \mathbf{H} \mathbf{~ N M R}\left(400 \mathrm{MHz}, \mathrm{CDCl}_{3}\right) \delta 9.72$ $(\mathrm{s}, 1 \mathrm{H}), 7.89-7.78(\mathrm{~m}, 4 \mathrm{H}), 7.56-7.44(\mathrm{~m}, 3 \mathrm{H}), 6.01(\mathrm{t}, J=6.7 \mathrm{~Hz}, 1 \mathrm{H}), 4.95-4.76(\mathrm{~m}, 2 \mathrm{H})$, $1.67(\mathrm{~s}, 3 \mathrm{H}), 1.10(\mathrm{~d}, J=1.3 \mathrm{~Hz}, 6 \mathrm{H}) .{ }^{13} \mathbf{C} \mathbf{~ N M R}\left(100 \mathrm{MHz}, \mathrm{CDCl}_{3}\right) \delta$ 207.8, 206.4, 141.1, 132.8, 131.9, 128.1, 127.2, 127.0, 126.9, 126.4, 126.1, 126.0, 95.9, 77.7, 51.4, 46.0, 22.0, 19.1, 18.9. IR $\left(\right.$ atr, $\left.\mathrm{cm}^{-1}\right) 3057,2978,2926,2852,2720,1953,1718,1598,1505,1461,1378,1276$, $1241,1178,1127,1089,948,852,819,745,477$. HRMS (ESI+, M+Na) Calc'd for $\mathrm{C}_{19} \mathrm{H}_{20} \mathrm{ONa}$ 287.1406, found 287.1403. Enantiomeric purity was determined by HPLC analysis in comparison with racemic material $\mathrm{T}_{\mathrm{R}}=14.9$ (minor) $\mathrm{T}_{\mathrm{R}}=22.1$ (major) (99:1 er shown); Chiracel OJ-H column, $90 \%$ hexane to $\left.10 \% \mathrm{iPrOH}, 1.0 \mathrm{~mL} / \mathrm{min}, 219 \mathrm{~nm}, 600 \mathrm{psi}, 25^{\circ} \mathrm{C}\right)[\boldsymbol{\alpha}]_{\boldsymbol{D}}^{\mathbf{2 5}}$ $=-159.7\left(\mathrm{c}=0.5, \mathrm{CHCl}_{3}\right)$.
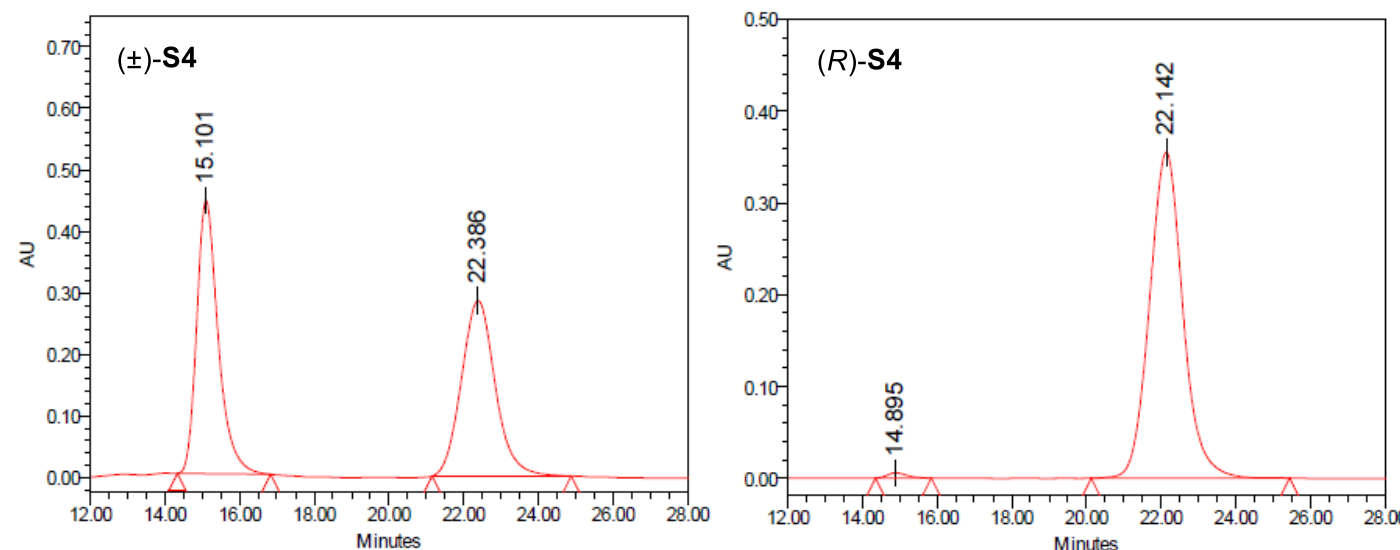

Peak Results
\begin{tabular}{|c|c|c|c|r|c|c|}
\hline & Name & RT & Area & $\%$ Area & Height & Units \\
\hline 1 & & 15.101 & 17790169 & 49.82 & 444006 & \\
\hline 2 & & 22.386 & 17919484 & 50.18 & 284966 & \\
\hline
\end{tabular}

Peak Results
\begin{tabular}{|l|c|c|r|r|r|r|}
\hline & Name & RT & Area & $\%$ Area & Height & Units \\
\hline 1 & & 14.895 & 205323 & 0.92 & 5756 & \\
\hline 2 & & 22.142 & 22088453 & 99.08 & 354661 & \\
\hline
\end{tabular}




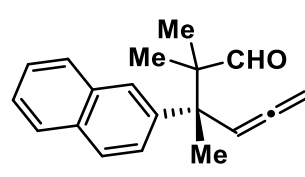

$(R)-\mathbf{S} 4$

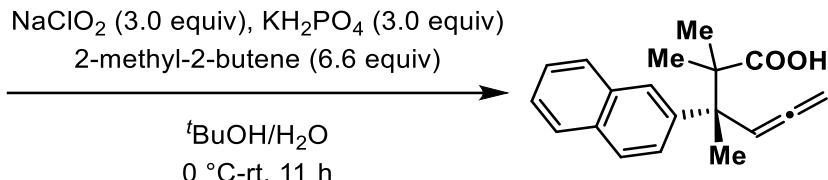

$(R)-7$

$(\boldsymbol{R})$-2,2,3-trimethyl-3-(naphthalen-2-yl)hexa-4,5-dienoic acid [(R)-7] - A dried $25 \mathrm{~mL}$ flask containing magnetic stir bar was charged with $(R)$-S4 $(370 \mathrm{mg}, 1.40 \mathrm{mmol}, 1$ equiv) and 2methyl-2-butene $\left(0.98 \mathrm{~mL}, 9.24 \mathrm{mmol}, 6.6\right.$ equiv). The mixture was taken up with ${ }^{t} \mathrm{BuOH}(3.0$ $\mathrm{mL})$ and was cooled to $0{ }^{\circ} \mathrm{C}$. To another $15 \mathrm{~mL}$ flask was charged with $\mathrm{NaClO}_{2}(475 \mathrm{mg}, 4.20$ mmol, 3.0 equiv) and $\mathrm{KH}_{2} \mathrm{PO}_{4}$ (571 $\mathrm{mg}$, 4.20 mmol, 3.0 equiv) which was dissolved in $\mathrm{H}_{2} \mathrm{O}(2$ $\mathrm{mL}$ ). This solution was taken via syringe and added to the aldehyde solution dropwise. After 5 minutes, the reaction was warmed to room temperature and was stirred for 11 hours. The reaction was concentrated in vacuo to remove ${ }^{t} \mathrm{BuOH}$, and diluted with $\mathrm{H}_{2} \mathrm{O}(10 \mathrm{~mL})$. The organics were extracted with EtOAc $\left(15 \mathrm{~mL}\right.$ x 3), dried over $\mathrm{Na}_{2} \mathrm{SO}_{4}$, filtered, and concentrated in vасио. The crude residue was purified via $\mathrm{SiO}_{2}$ flash column chromatography using pentane:Et ${ }_{2} \mathrm{O}(4: 1 \mathrm{v}: \mathrm{v})$, and the title compound was isolated as a white solid (327 $\mathrm{mg}, 1.17$ mmol, 83\% yield, $\left.\mathrm{MP}=92.9-93.3{ }^{\circ} \mathrm{C}\right) .{ }^{1} \mathbf{H}$ NMR $\left(400 \mathrm{MHz}, \mathrm{CDCl}_{3}\right) \delta 7.87-7.70(\mathrm{~m}, 4 \mathrm{H})$, $7.60-7.55(\mathrm{~m}, 1 \mathrm{H}), 7.48-7.42(\mathrm{~m}, 2 \mathrm{H}), 6.27(\mathrm{t}, J=6.7 \mathrm{~Hz}, 1 \mathrm{H}), 4.94-4.56(\mathrm{~m}, 2 \mathrm{H}), 1.73(\mathrm{~s}$, $3 \mathrm{H}), 1.22(\mathrm{~d}, J=5.6 \mathrm{~Hz}, 6 \mathrm{H}) .{ }^{13} \mathbf{C} \mathbf{N M R}\left(100 \mathrm{MHz}, \mathrm{CDCl}_{3}\right) \delta 207.9,182.5,141.6,132.7,132.0$, 128.2, 127.2, 127.1, 126.6, 125.8, 125.8, 96.6, 77.2, 76.7, 49.6, 46.5, 22.4, 22.3, 22.3. IR (atr, $\mathrm{cm}^{-1}$ ) 3057, 2979, 2652, 2555, 1954, 1690, 1598, 1506, 1472, 1380, 1287, 1188, 1126, 1087, 1017, 947, 849, 817, 743, 555, 476. HRMS (ESI-, M-H) Calc'd for $\mathrm{C}_{19} \mathrm{H}_{19} \mathrm{O}_{2} 279.1391$, found 279.1395. Enantiomeric purity was determined by HPLC analysis in comparison with racemic material $\mathrm{T}_{\mathrm{R}}=6.6$ (minor) $\mathrm{T}_{\mathrm{R}}=7.1$ (major) (99:1 er shown); Chiralcel IC column, 98\% hexane to $2 \%$ iPrOH, $\left.1.0 \mathrm{~mL} / \mathrm{min}, 225 \mathrm{~nm}, 485 \mathrm{psi}, 25^{\circ} \mathrm{C}\right)[\boldsymbol{\alpha}]_{D}^{25}=-1.0\left(\mathrm{c}=0.5, \mathrm{CHCl}_{3}\right)$.
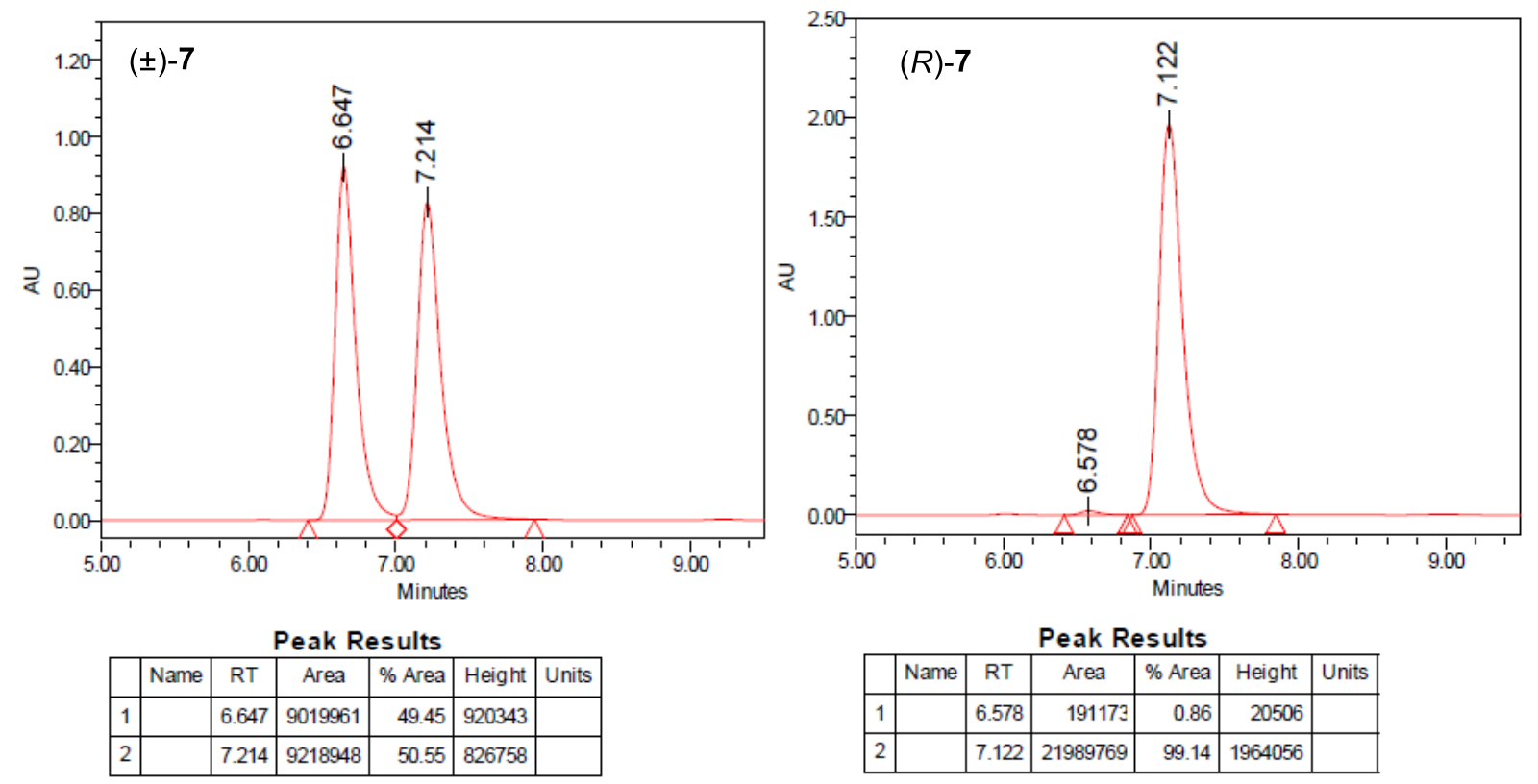


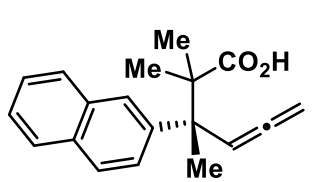

$(R)-7$

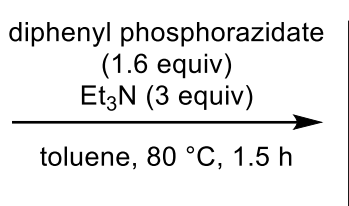$$
\text { . }
$$

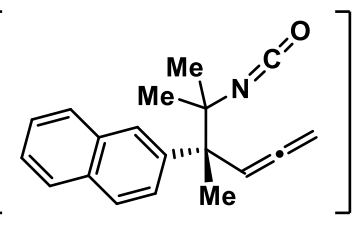

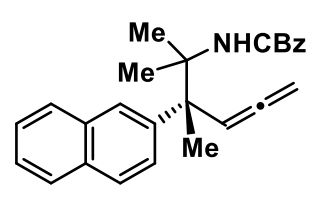

$(R)-8$

benzyl $(R)$-(2,3-dimethyl-3-(naphthalen-2-yl)hexa-4,5-dien-2-yl)carbamate $[(R)-8]$ - A dried $1 \mathrm{~mL}$ vial containing a magnetic stir-bar was charged with $(R)-7(56.1 \mathrm{mg}, 0.2 \mathrm{mmol}, 1$ equiv) which was taken up with toluene $(1 \mathrm{~mL})$. To this solution was added $\mathrm{Et}_{3} \mathrm{~N}(84 \mu \mathrm{L}, 0.6$ mmol, 3.0 equiv) and diphenyl phosphorazidate $(68.8 \mu \mathrm{L}, 0.32 \mathrm{mmol}, 1.6$ equiv). The vial was purged with dried Ar and then sealed with a screw cap. The mixture was stirred at $80{ }^{\circ} \mathrm{C}$ for 1.5 hours. After cooling to room temperature, benzyl alcohol (146 $\mu \mathrm{L}, 1.4 \mathrm{mmol}, 7.0$ equiv) and $\mathrm{Et}_{3} \mathrm{~N}$ (195 $\mu \mathrm{L}, 1.4 \mathrm{mmol}, 7.0$ equiv) were added. The vial was re-purged with dried Ar, sealed with a screw cap, and the reaction was stirred at $80{ }^{\circ} \mathrm{C}$ for 12 hours. The reaction mixture was then passed through a plug of Celite eluting with $\mathrm{Et}_{2} \mathrm{O}$. The filtrate was concentrated in vacuo and the crude residue was purified by $\mathrm{SiO}_{2}$ flash column chromatography using pentane:Et $2 \mathrm{O}$ $(10: 1 \mathrm{v}: \mathrm{v})$. The title compound was obtained as a clear colorless oil $(39.4 \mathrm{mg}, 0.10 \mathrm{mmol}, 51 \%$ yield). ${ }^{1} \mathbf{H}$ NMR $\left(400 \mathrm{MHz}, \mathrm{CDCl}_{3}\right) \delta 7.91-7.87(\mathrm{~m}, 1 \mathrm{H}), 7.86-7.76(\mathrm{~m}, 3 \mathrm{H}), 7.64-7.59$ $(\mathrm{m}, 1 \mathrm{H}), 7.53-7.45(\mathrm{~m}, 2 \mathrm{H}), 7.42-7.31(\mathrm{~m}, 5 \mathrm{H}), 6.12(\mathrm{t}, J=6.7 \mathrm{~Hz}, 1 \mathrm{H}), 5.05(\mathrm{~s}, 2 \mathrm{H}), 4.91$ $-4.73(\mathrm{~m}, 3 \mathrm{H}), 1.68$ (s, 3H), 1.48 (s, 3H), 1.40 (s, 3H). $\left.{ }^{13} \mathbf{C ~ N M R ~ ( 1 0 0 ~ M H z , ~} \mathrm{CDCl}_{3}\right) \delta 208.1$, 155.0, 141.2, 136.9, 132.7, 131.9, 128.4 (2C), 128.2, 128.0 (2C), 128.0, 127.5, 127.2, 126.9, 126.9, 126.0, 125.9, 96.2, 77.3, 66.0, 58.8, 49.6, 23.9, 23.6, 21.7. IR (atr, $\left.\mathrm{cm}^{-1}\right) 3410,3061$, 2984, 1953, 1729, 1502, 1455, 1379, 1245, 1069, 850, 819, 744, 697, 477. HRMS (ESI+, $\mathrm{M}+\mathrm{Na}$ ) Calc'd for $\mathrm{C}_{26} \mathrm{H}_{27} \mathrm{NO}_{2} \mathrm{Na}$ 408.1934, found 408.1934. Enantiomeric purity was determined by HPLC analysis in comparison with racemic material $\mathrm{T}_{\mathrm{R}}=11.9$ (minor) $\mathrm{T}_{\mathrm{R}}=14.3$ (minor) (99:1 er shown); Chiracel OD-H column, 98\% hexane to $2 \% \mathrm{iPrOH}, 1.0 \mathrm{~mL} / \mathrm{min}, 225$ $\left.\mathrm{nm}, 525 \mathrm{psi}, 25^{\circ} \mathrm{C}\right)[\boldsymbol{\alpha}]_{\boldsymbol{D}}^{\mathbf{2 4}}=-105.1\left(\mathrm{c}=0.5, \mathrm{CHCl}_{3}\right)$.
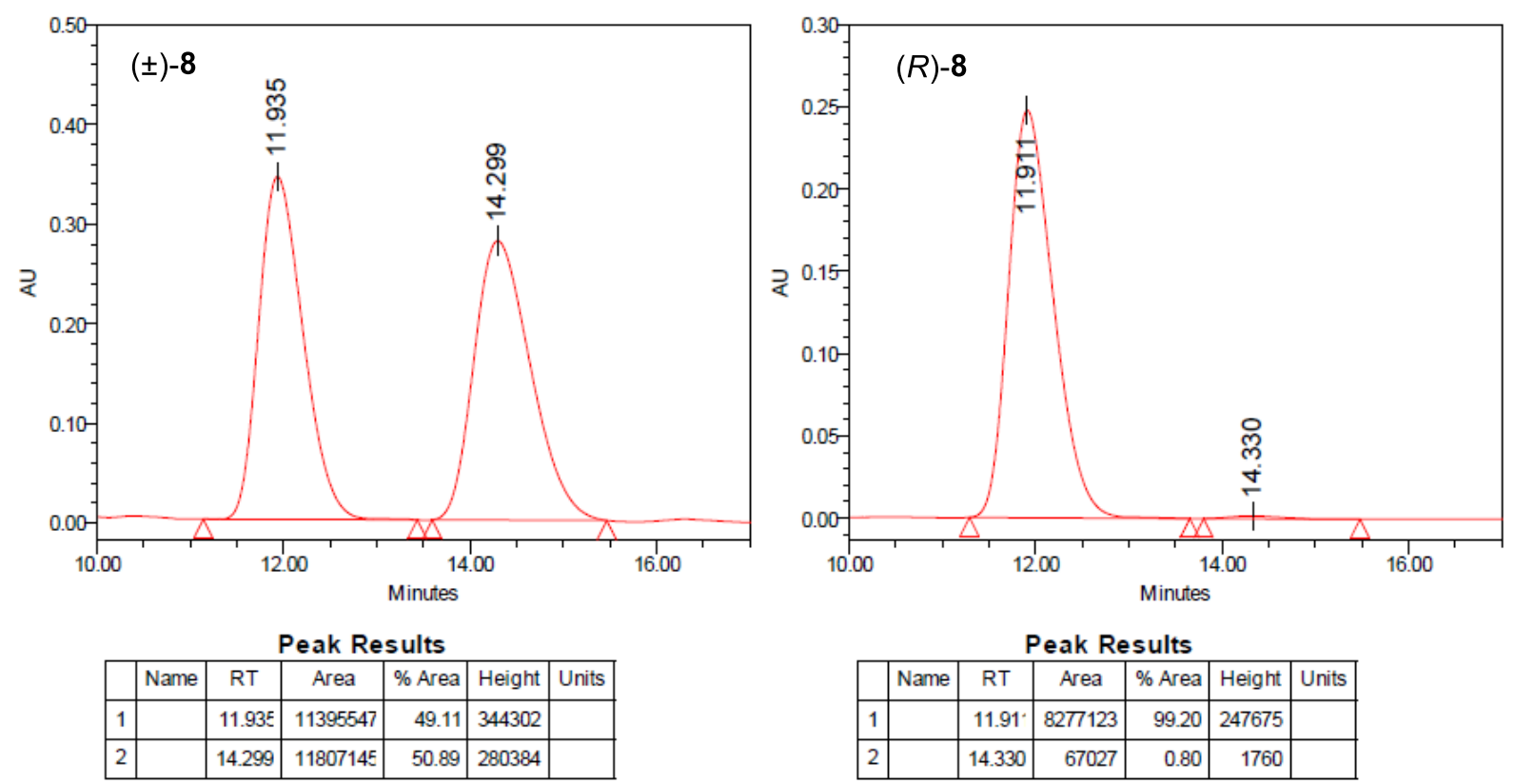

Breit's Rh-catalyzed intramolecular cyclization ${ }^{19}$ 


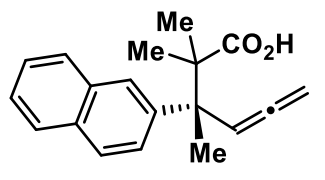

$(R)-7$
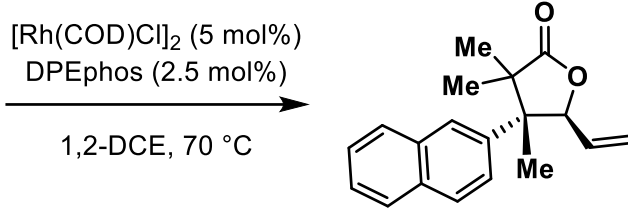

$(R, S)-9 ; 5.2: 1 \mathrm{dr}$ [X-ray]

$(4 R, 5 S)-3,3,4-t r i m e t h y l-4-(n a p h t h a l e n-2-y l)-5$-vinyldihydrofuran-2(3H)-one $[(R, S)-9]-\mathrm{A}$ dried $1 \mathrm{~mL}$ screw vial containing a magnetic stir-bar was charged with $(R)-7(56.1 \mathrm{mg}, 0.2$ mmol, 1 equiv), $[\mathrm{Rh}(\operatorname{cod}) \mathrm{Cl}]_{2}(2.47 \mathrm{mg}, 5 \mu \mathrm{mol}, 2.5 \mathrm{~mol} \%)$, and DPEphos (5.39 $\mathrm{mg}, 10 \mu \mathrm{mol}$, $5.0 \mathrm{~mol} \%)$, which were taken up with 1,2-DCE $(2.0 \mathrm{~mL})$. The vial was purged with dried Ar and then sealed with a screw cap. The mixture was stirred at $70{ }^{\circ} \mathrm{C}$ for 5 hours. The reaction was passed through a plug of $\mathrm{SiO}_{2}$ eluting with DCM and EtOAc, and the filtrate was concentrated in vacuo. The diastereomeric ratio (dr) and was determined to be $5.2: 1$ by ${ }^{1} \mathrm{H} \mathrm{NMR}$ analysis of the unpurified reaction mixture. The crude residue was purified by $\mathrm{SiO}_{2}$ flash column chromatography using pentane:EtOAc:EtOH (60:3:1 v:v:v). The title compound was obtained as a clear colorless oil $(44.4 \mathrm{mg}, 0.16 \mathrm{mmol}, 79 \%$ yield). X-ray quality crystals of $(R, S)-9$ could be obtained by vapor diffusion using $\mathrm{Et}_{2} \mathrm{O}$ (solvent) and pentanes (anti-solvent) cooled to $4{ }^{\circ} \mathrm{C} .{ }^{1} \mathbf{H}$ NMR $\left(400 \mathrm{MHz}, \mathrm{CDCl}_{3}\right) \delta 7.91-7.81(\mathrm{~m}, 3 \mathrm{H}), 7.77-7.70(\mathrm{~m}, 1 \mathrm{H}), 7.56$ $-7.48(\mathrm{~m}, 2 \mathrm{H}), 7.46(\mathrm{dd}, J=8.7,2.1 \mathrm{~Hz}, 1 \mathrm{H}), 6.00(\mathrm{ddd}, J=17.3,10.9,5.0 \mathrm{~Hz}, 1 \mathrm{H}), 5.78$ (dt, $J=5.1,1.6 \mathrm{~Hz}, 1 \mathrm{H}), 5.67(\mathrm{dt}, J=17.3,1.5 \mathrm{~Hz}, 1 \mathrm{H}), 5.42(\mathrm{dt}, J=10.9,1.5 \mathrm{~Hz}, 1 \mathrm{H}), 1.39(\mathrm{~d}, J$ $=3.2 \mathrm{~Hz}, 6 \mathrm{H}), 0.89$ (s, 3H). ${ }^{13} \mathbf{C}$ NMR $\left(100 \mathrm{MHz}, \mathrm{CDCl}_{3}\right) \delta 180.5,137.7,133.1,132.2,131.0$, $128.4,128.0,127.4,126.4,126.3,125.0,124.2,119.0,80.9,51.8,48.3,22.2,19.7,19.3$. IR $\left(\right.$ atr, $\left.\mathrm{cm}^{-1}\right)$ 3062, 2976, 2934, 1771, 1599, 1507, 1460, 1392, 1283, 1190, 1148, 1099, 1015, 992, 852, 818, 748, 476. HRMS (ESI+, M+Na) Calc'd for $\mathrm{C}_{19} \mathrm{H}_{20} \mathrm{O}_{2} \mathrm{Na} 303.1356$, found 303.1353. Enantiomeric purity was determined by HPLC analysis in comparison with racemic material $\mathrm{T}_{\mathrm{R}}=19.1$ (minor) $\mathrm{T}_{\mathrm{R}}=21.7$ (minor) (99:1 er shown); Chiracel IC column, 95\% hexane to 5\% $\left.\mathrm{iPrOH}, 1.0 \mathrm{~mL} / \mathrm{min}, 222 \mathrm{~nm}, 500 \mathrm{psi}, 26{ }^{\circ} \mathrm{C}\right)[\boldsymbol{\alpha}]_{\boldsymbol{D}}^{25}=-275.0\left(\mathrm{c}=0.5, \mathrm{CHCl}_{3}\right)$. The relative stereochemistry of this compound was assigned by X-ray crystallographic analysis.
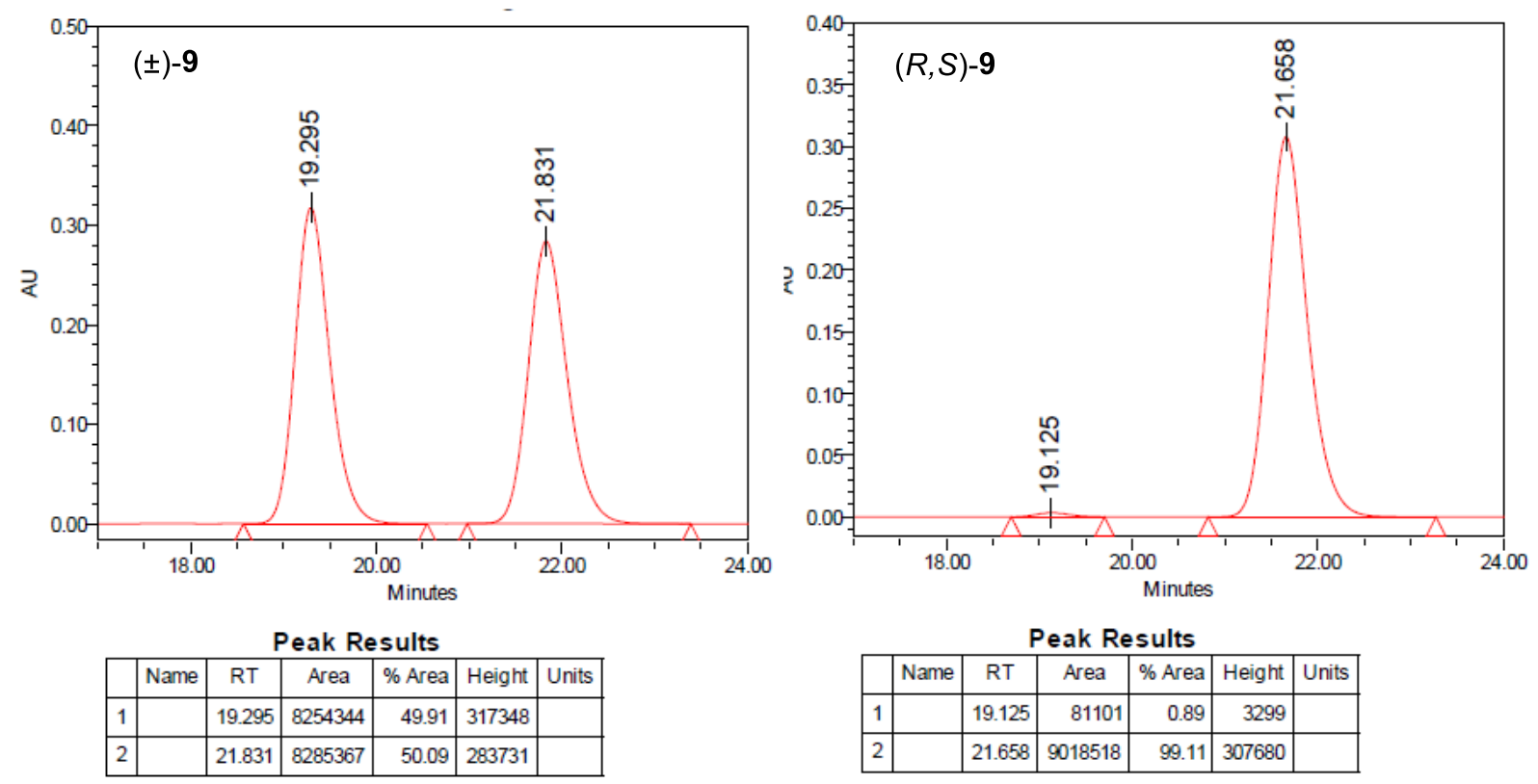
Characterization data for the minor diastereomer $(4 R, 5 R)-3,3,4$-trimethyl-4-(naphthalen2-yl)-5-vinyldihydrofuran-2(3H)-one $[(R, R)-9]$ :

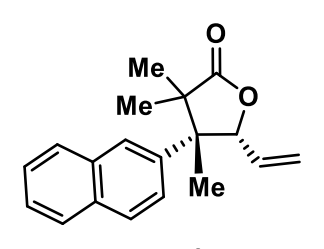

$(R, R)-9$

The title compound was obtained as a clear colorless oil $(8.4 \mathrm{mg}, 0.03$ mmol, $15 \%$ yield). ${ }^{1} \mathbf{H}$ NMR $\left(400 \mathrm{MHz}, \mathrm{CDCl}_{3}\right) \delta 7.85-7.73(\mathrm{~m}, 3 \mathrm{H})$, $7.69-7.65(\mathrm{~m}, 1 \mathrm{H}), 7.52-7.44(\mathrm{~m}, 2 \mathrm{H}), 7.41(\mathrm{dd}, J=8.8,2.2 \mathrm{~Hz}, 1 \mathrm{H})$, 5.61 (ddd, $J=17.2,10.5,6.9 \mathrm{~Hz}, 1 \mathrm{H}), 5.40$ (dt, $J=17.1,1.3 \mathrm{~Hz}, 1 \mathrm{H}), 5.13$ $(\mathrm{dt}, J=10.5,1.2 \mathrm{~Hz}, 1 \mathrm{H}), 4.96(\mathrm{dt}, J=6.8,1.2 \mathrm{~Hz}, 1 \mathrm{H}), 1.58(\mathrm{~s}, 3 \mathrm{H}), 1.39$ $(\mathrm{s}, 3 \mathrm{H}), 0.75(\mathrm{~s}, 3 \mathrm{H}) .{ }^{13} \mathrm{C}$ NMR $\left(100 \mathrm{MHz}, \mathrm{CDCl}_{3}\right) \delta 182.3,137.8,133.1,132.2,131.9,128.2$, 127.8, 127.3, 126.4, 126.2 (2C), 125.6, 120.1, 87.6, 52.4, 48.0, 22.8, 20.2, 17.5. IR (atr, cm ${ }^{-1}$ ) 3062, 2976, 2924, 2855, 1776, 1600, 1506, 1457, 1393, 1380, 1318, 1266, 1136, 1013, 985, 863, 781, 746, 477. IR (atr, $\left.\mathrm{cm}^{-1}\right)$ 3062, 2979, 2924, 2855, 1776, 1600, 1506, 1457, 1393, 1380, $1318,1136,1013,985,863,820,781,746,477$. HRMS (ESI+, M+Na) Calc'd for $\mathrm{C}_{19} \mathrm{H}_{20} \mathrm{O}_{2} \mathrm{Na}$ 303.1356, found 303.1355. $[\alpha]_{D}^{\mathbf{2 4}}=+97.7\left(\mathrm{c}=0.5, \mathrm{CHCl}_{3}\right)$. 
Determination of absolute configuration for the products obtained via enantioselective Ircatalyzed allenylic alkylation: X-ray crystallographic analysis of $\mathbf{S 5}$ :

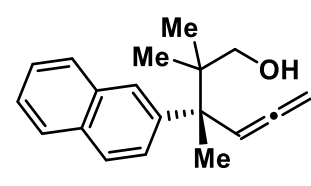

$(R)-\mathbf{S 3}$
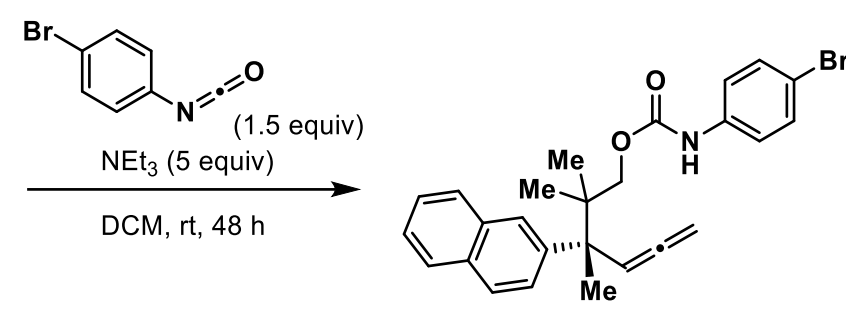

(R)-S5

[X-ray]

$(R)$-2,2,3-trimethyl-3-(naphthalen-2-yl)hexa-4,5-dien-1-yl (4-bromophenyl)carbamate [(R)-S5] - A dried $10 \mathrm{~mL}$ round bottom flask containing a magnetic stir-bar was charged with DCM solution of $(R)-\mathbf{S 3}(25.0 \mathrm{mg}, 94 \mu \mathrm{mol}, 1$ equiv, $0.1 \mathrm{M}), p$-bromophenylisocyanate $(27.9$ $\mathrm{mg}, 0.14 \mathrm{mmol}, 1.5$ equiv) and $\mathrm{NEt}_{3}(65.4 \mu \mathrm{L}, 0.47 \mathrm{mmol}, 5$ equiv) in that order. The resulting heterogeneous solution was stirred at room temperature for 48 hours. The solution was filtered through a short plug of Celite and the filtrate was concentrated in vacuo. The crude residue was purified via $\mathrm{SiO}_{2}$ flash column chromatography using pentane:Et ${ }_{2} \mathrm{O}(4: 1 \mathrm{v}: \mathrm{v})$, and the title compound was isolated as a white solid $\left(30.4 \mathrm{mg}, 65 \mu \mathrm{mol}, 70 \%\right.$ yield, $\mathrm{MP}=109.3-109.5^{\circ} \mathrm{C}$ ). $\mathrm{X}$-ray quality crystals of $(R)$-S5 were obtained by vapor diffusion using toluene (solvent) and pentanes (anti-solvent) at $4{ }^{\circ} \mathrm{C}$ in a cold room. ${ }^{1} \mathbf{H}$ NMR $\left(400 \mathrm{MHz}, \mathrm{CDCl}_{3}\right) \delta 7.86-7.71(\mathrm{~m}$, $4 \mathrm{H}), 7.60-7.54(\mathrm{~m}, 1 \mathrm{H}), 7.50-7.41(\mathrm{~m}, 2 \mathrm{H}), 7.40-7.33(\mathrm{~m}, 2 \mathrm{H}), 7.16(\mathrm{~d}, J=8.4 \mathrm{~Hz}, 2 \mathrm{H})$, $6.29(\mathrm{br}, 1 \mathrm{H}), 6.12(\mathrm{t}, J=6.7 \mathrm{~Hz}, 1 \mathrm{H}), 4.86-4.61(\mathrm{~m}, 2 \mathrm{H}), 4.10(\mathrm{q}, J=10.8 \mathrm{~Hz}, 2 \mathrm{H}), 1.65(\mathrm{~s}$, $3 \mathrm{H}), 1.03(\mathrm{~d}, J=4.5 \mathrm{~Hz}, 6 \mathrm{H}) .{ }^{13} \mathbf{C}$ NMR $\left(100 \mathrm{MHz}, \mathrm{CDCl}_{3}\right) \delta 207.8,153.3,142.5,136.9,132.7$, 131.9 (2C), 131.7, 128.2, 127.2, 127.2, 127.1, 126.4, 125.9, 125.8, 120.0, 115.8, 96.7, 77.2, 71.3, 46.4, 40.9, 21.8, 21.5, 21.4. IR (atr, $\left.\mathrm{cm}^{-1}\right)$ 3407, 3318, 3057, 2977, 2924, 2853, 1953, 1703, 1595, 1521, 1490, 1401, 1307, 1219, 1075, 1056, 1008, 854, 822, 743, 503, 477. HRMS $\left(\mathrm{ESI}+\mathrm{M}+\mathrm{Na}\right.$ ) Calc'd for $\mathrm{C}_{26} \mathrm{H}_{26} \mathrm{BrNO}_{2} \mathrm{Na} 486.1039$, found 486.1041. $[\boldsymbol{\alpha}]_{\boldsymbol{D}}^{\mathbf{2 6}}=-71.0(\mathrm{c}=0.5$, $\left.\mathrm{CHCl}_{3}\right)$. 
Determination of absolute configuration of products obtained via enantioselective Ir-catalyzed allenylic alkylation: $X$-ray crystallographic analysis of $\mathbf{S 8 :}$

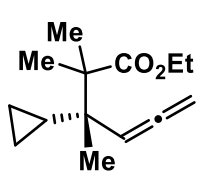

(S)-3q

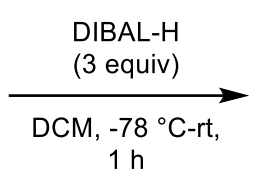

$1 \mathrm{~h}$

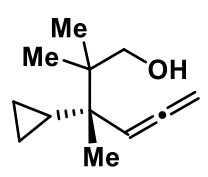

(S)-S6

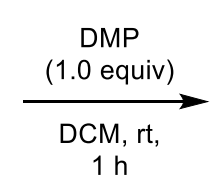

$\mathrm{h}$

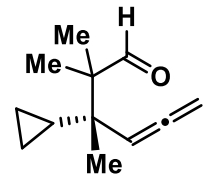

(S)-S7

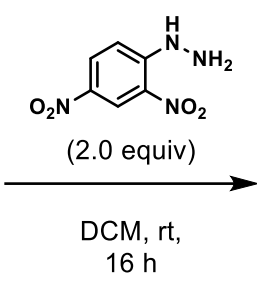

$6 \mathrm{~h}$

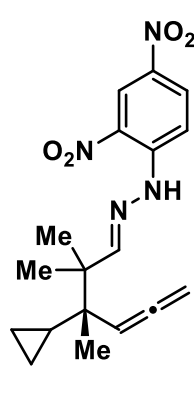

(S)-S8

(S)-3-cyclopropyl-2,2,3-trimethylhexa-4,5-dien-1-ol [(S)-S6] - A dried $10 \mathrm{~mL}$ flask containing magnetic stir bar was charged with $(S)-3 q(59 \mathrm{mg}, 0.27 \mathrm{mmol}, 1$ equiv) which was taken up with DCM $(3.0 \mathrm{~mL})$. The mixture was cooled to $-78^{\circ} \mathrm{C}$ and DIBAL-H $(0.80 \mathrm{~mL}, 0.80$ mmol, $1 \mathrm{M}$ in toluene, 3 equiv) was added dropwise over 3 minutes. The resulting mixture was warmed to room temperature and stirred for 30 minutes. The reaction was cooled to $0{ }^{\circ} \mathrm{C}$ and quenched with saturated Rochelle's Salt. The resulting biphasic mixture was stirred vigorously for 30 minutes. The organics were extracted with DCM (3 x $5 \mathrm{~mL})$ and the combined organic layers were washed with brine, dried over $\mathrm{Na}_{2} \mathrm{SO}_{4}$, filtered and concentrated in vacuo. The crude residue was purified via $\mathrm{SiO}_{2}$ flash column chromatography using pentane:Et $t_{2} \mathrm{O}(5: 1 \mathrm{v}: \mathrm{v})$. The title compound was isolated as a colorless oil (40 mg, $0.22 \mathrm{mmol}, 84 \%$ yiled). ${ }^{1} \mathbf{H}$ NMR $\left(400 \mathrm{MHz}, \mathrm{CDCl}_{3}\right) \delta 5.06(\mathrm{t}, J=6.7 \mathrm{~Hz}, 1 \mathrm{H}), 4.76-4.63(\mathrm{~m}, 2 \mathrm{H}), 3.64-3.49(\mathrm{~m}, 2 \mathrm{H}), 1.50$ (s, 1H), $1.05-0.83(\mathrm{~m}, 1 \mathrm{H}), 1.00(\mathrm{~s}, 3 \mathrm{H}), 0.97(\mathrm{~s}, 3 \mathrm{H}), 0.88(\mathrm{~s}, 3 \mathrm{H}), 0.42-0.22(\mathrm{~m}, 3 \mathrm{H}), 0.21$ $-0.12(\mathrm{~m}, 1 \mathrm{H}) .{ }^{13} \mathbf{C} \mathbf{N M R}\left(100 \mathrm{MHz}, \mathrm{CDCl}_{3}\right) \delta 208.2,93.8,75.8,69.8,41.9,41.3,20.8,20.6$, 18.6, 17.0, 2.0, 0.7. IR (atr, cm $\left.{ }^{-1}\right) 3366,3078,2974,2880,1953,1459,1376,1102,1022,947$, 883, 838, 641. HRMS (EI+, M+) Calc'd for $\mathrm{C}_{12} \mathrm{H}_{20} \mathrm{O} 180.1509$, found 180.1507. $[\boldsymbol{\alpha}]_{\boldsymbol{D}}^{\mathbf{2 6}}=-43.5$ $\left(\mathrm{c}=0.4, \mathrm{CHCl}_{3}\right)$.

(S)-3-cyclopropyl-2,2,3-trimethylhexa-4,5-dienal [(S)-S7] - A dried $25 \mathrm{~mL}$ flask containing magnetic stir bar was charged with $(S)$-S6 $(20 \mathrm{mg}, 0.11 \mathrm{mmol}, 1$ equiv) which was taken up with DCM (5.5 mL). The solution was cooled to $0{ }^{\circ} \mathrm{C}$ and DMP $(47.1 \mathrm{mg}, 0.11 \mathrm{mmol}, 1.0$ equiv) was added. The reaction was stirred for 1 hour maintaining the temperature. The reaction was diluted $\mathrm{Et}_{2} \mathrm{O}(5 \mathrm{~mL})$, filtered through a plug of $\mathrm{SiO}_{2}$ eluting with $\mathrm{Et}_{2} \mathrm{O}(5 \mathrm{~mL})$, and the filtrate was concentrated in vacuo. The crude residue was purified via $\mathrm{SiO}_{2}$ flash column chromatography using pentane: $\mathrm{Et}_{2} \mathrm{O}(20: 1 \mathrm{v}: \mathrm{v})$, and the title compound was isolated as a white solid (19 mg, $0.11 \mathrm{mmol}, 96 \%$ yield). ${ }^{1} \mathbf{H}$ NMR (400 MHz, $\left.\mathrm{CDCl}_{3}\right) \delta 9.80(\mathrm{~s}, 1 \mathrm{H}), 4.98(\mathrm{t}, J=$ $6.6 \mathrm{~Hz}, 1 \mathrm{H}), 4.75(\mathrm{dd}, J=6.6,1.1 \mathrm{~Hz}, 2 \mathrm{H}), 1.14(\mathrm{~s}, 3 \mathrm{H}), 1.11(\mathrm{~s}, 3 \mathrm{H}), 1.01-0.94(\mathrm{~m}, 1 \mathrm{H}), 0.93$ $(\mathrm{s}, 3 \mathrm{H}), 0.46-0.26(\mathrm{~m}, 4 \mathrm{H}), 0.22-0.15(\mathrm{~m}, 1 \mathrm{H}) .{ }^{13} \mathbf{C ~ N M R}\left(100 \mathrm{MHz}, \mathrm{CDCl}_{3}\right) \delta 208.6,207.2$, 92.5, 76.5, 52.1, 41.4, 18.7, 18.4, 18.4, 16.9, 2.0, 0.8. IR (atr, $\left.\mathrm{cm}^{-1}\right)$ 3081, 2976, 2936, 2717 , 1954, 1721, 1461, 1400, 1378, 1101, 1050, 1021, 948, 907, 883, 842, 788. HRMS (EI+, M-H) Calc'd for $\mathrm{C}_{12} \mathrm{H}_{17} \mathrm{O}$ 177.1274, found 177.1272. $[\boldsymbol{\alpha}]_{D}^{25}=-47.6\left(\mathrm{c}=0.5, \mathrm{CHCl}_{3}\right)$.

\section{(S)-1-(3-cyclopropyl-2,2,3-trimethylhexa-4,5-dien-1-ylidene)-2-(2,4-}

dinitrophenyl)hydrazine $[(S)$-S8 $]$ - A $10 \mathrm{~mL}$ round bottom flask containing a magnetic stir bar was charged with $(S)$-S7 (13 mg, $73 \mu \mathrm{mol}, 1$ equiv) and (2,4-dinitrophenyl)hydrazine (28.9 $\mathrm{mg}, 0.146 \mathrm{mmol}, 2.0$ equiv) which was taken up with DCM $(1.0 \mathrm{~mL})$. The flask was purged with $\mathrm{N}_{2}$ and closed with a rubber septum. The reaction was stirred at room temperature for 16 hours. The reaction mixture filtered through a short plug of Celite eluting with DCM. Following 
concentration of the filtrate, the crude residue was purified via $\mathrm{SiO}_{2}$ flask column chromatography using pentane: $\mathrm{Et}_{2} \mathrm{O}(20: 1 \mathrm{v}: \mathrm{v})$ as the mobile phase. The title compound was obtained as a yellow solid $\left(18.8 \mathrm{mg}, 52 \mu \mathrm{mol}, 72 \%\right.$ yield, $\left.\mathrm{MP}=119.5-120.4{ }^{\circ} \mathrm{C}\right)$. X-ray quality crystals of $(S)$-S8 were obtained by vapor diffusion using $\mathrm{Et}_{2} \mathrm{O}$ (solvent) and pentanes (antisolvent) at $4{ }^{\circ} \mathrm{C}$ in a cold room. ${ }^{1} \mathbf{H}$ NMR $\left(400 \mathrm{MHz}, \mathrm{CDCl}_{3}\right) \delta 10.99(\mathrm{~s}, 1 \mathrm{H}), 9.12(\mathrm{~d}, J=2.6$ $\mathrm{Hz}, 1 \mathrm{H}), 8.30(\mathrm{dd}, J=9.7,2.4 \mathrm{~Hz}, 1 \mathrm{H}), 7.94(\mathrm{~d}, J=9.6 \mathrm{~Hz}, 1 \mathrm{H}), 7.67(\mathrm{~s}, 1 \mathrm{H}), 4.98(\mathrm{t}, J=6.6$ $\mathrm{Hz}, 1 \mathrm{H}), 4.82-4.70(\mathrm{~m}, 2 \mathrm{H}), 1.28(\mathrm{~s}, 6 \mathrm{H}), 1.01-0.95(\mathrm{~m}, 1 \mathrm{H}), 0.94(\mathrm{~s}, 3 \mathrm{H}), 0.47-0.34(\mathrm{~m}$, $2 \mathrm{H}), 0.33-0.25(\mathrm{~m}, 1 \mathrm{H}), 0.23-0.14(\mathrm{~m}, 1 \mathrm{H}) .{ }^{13} \mathbf{C} \mathbf{~ N M R}\left(100 \mathrm{MHz}, \mathrm{CDCl}_{3}\right) \delta 208.6,158.9$, 145.3, 137.7, 129.9, 128.8, 123.5, 116.7, 92.8, 76.5, 45.6, 42.6, 21.8, 21.5, 18.9, 17.0, 2.1, 0.8. IR $\left(\right.$ atr, $\left.\mathrm{cm}^{-1}\right)$ 3299, 3109, 2977, 2937, 1953, 1617, 1590, 1518, 1424, 1331, 1308, 1277, 1221, 1137, 1070, 1046, 923, 833, 743. HRMS (ESI+, M+Na) Calc'd for $\mathrm{C}_{18} \mathrm{H}_{22} \mathrm{~N}_{4} \mathrm{O}_{4} \mathrm{Na} 381.1533$, found 381.1537. $[\alpha]_{D}^{24}=+94.6\left(\mathrm{c}=0.5, \mathrm{CHCl}_{3}\right)$. 
Determination of absolute configuration of products obtained via enantioselective Ir-catalyzed allenylic alkylation: $X$-ray crystallographic analysis of $\mathbf{S 1 0 :}$

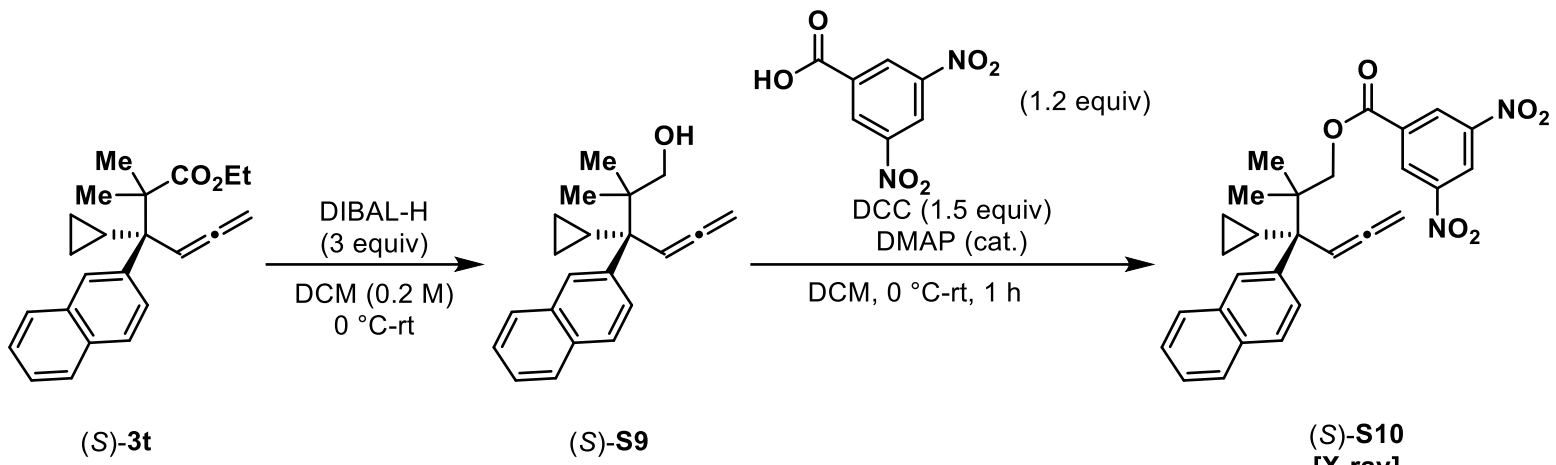

(S)-3-cyclopropyl-2,2-dimethyl-3-(naphthalen-2-yl)hexa-4,5-dien-1-ol [(S)-S9] - DIBAL-H $(90 \mu \mathrm{L}, 0.09 \mathrm{mmol}, 3$ equiv, $1 \mathrm{M}$ in toluene) was added dropwise to a DCM solution of $(S)-3 \mathrm{t}$ (10.0 mg, $30 \mu \mathrm{mol}, 1$ equiv, $0.2 \mathrm{M}, 95 \%$ ee) at $0{ }^{\circ} \mathrm{C}$ and the resulting solution was warmed to room temperature and stirred for 2 hours. The solution was re-cooled to $0{ }^{\circ} \mathrm{C}$ where it was quenched with a saturated aqueous solution of Rochelle's salt. The resulting biphasic mixture was stirred vigorously for 30 minutes until clean phase separation as observed. The layers were separated, and the aqueous layer was extracted with DCM $(3 \times 5 \mathrm{~mL})$ and the combined organic layers were washed with brine, dried over $\mathrm{Na}_{2} \mathrm{SO}_{4}$, filtered and concentrated in vacuo. The crude residue was purified via $\mathrm{SiO}_{2}$ flash column chromatography using hexanes:EtOAc (3:1 v:v) and was isolated as a clear and colorless oil (8.6 mg, $0.029 \mathrm{mmol}, 98 \%$ yield). ${ }^{1} \mathbf{H}$ NMR $\left(300 \mathrm{MHz}, \mathrm{CDCl}_{3}\right) \delta 8.01-7.94(\mathrm{~m}, 1 \mathrm{H}), 7.85-7.77(\mathrm{~m}, 2 \mathrm{H}), 7.74(\mathrm{~d}, J=8.8 \mathrm{~Hz}, 1 \mathrm{H}), 7.67$ $(\mathrm{dd}, J=8.8,2.0 \mathrm{~Hz}, 1 \mathrm{H}), 7.45(\mathrm{ddd}, J=5.4,4.5,3.3 \mathrm{~Hz}, 2 \mathrm{H}), 5.34(\mathrm{t}, J=6.8 \mathrm{~Hz}, 1 \mathrm{H}), 4.63(\mathrm{dd}$, $J=10.7,6.9 \mathrm{~Hz}, 1 \mathrm{H}), 4.47(\mathrm{dd}, J=10.7,6.7 \mathrm{~Hz}, 1 \mathrm{H}), 3.68-3.50(\mathrm{~m}, 2 \mathrm{H}), 1.86-1.73(\mathrm{~m}, 1 \mathrm{H})$, $1.55(\mathrm{~s}, 1 \mathrm{H}), 1.07(\mathrm{~s}, 3 \mathrm{H}), 1.02(\mathrm{~s}, 3 \mathrm{H}), 0.75-0.62(\mathrm{~m}, 2 \mathrm{H}), 0.61-0.50(\mathrm{~m}, 1 \mathrm{H}), 0.10--0.02$ $(\mathrm{m}, 1 \mathrm{H}) .{ }^{13} \mathbf{C}$ NMR $\left(100 \mathrm{MHz}, \mathrm{CDCl}_{3}\right) \delta 208.9,141.6,132.6,131.9,129.1,128.8,128.2,127.2$, 125.7, 125.6, 125.5, 90.5, 76.9, 69.8, 52.4, 43.1, 21.5, 21.4, 16.0, 5.3, 0.7. IR (atr, cm $\left.{ }^{-1}\right) 3388$, 3057, 2972, 2879, 1952, 1630, 1597, 1505, 1473, 1366, 1352, 1275, 1172, 1122, 1027, 997, 963, 899, 854, 821, 838, 743, 679. HRMS (ESI+, M+Na) Calc'd for $\mathrm{C}_{21} \mathrm{H}_{24} \mathrm{NaO} 315.1719$, found 315.1726. $[\alpha]_{D}^{23}=+32.2\left(\mathrm{c}=0.42, \mathrm{CHCl}_{3}\right)$.

\section{(S)-3-cyclopropyl-2,2-dimethyl-3-(naphthalen-2-yl)hexa-4,5-dien-1-yl}

dinitrobenzoate $[(S)$-S10] - A $1 \mathrm{~mL}$ screw-cap vial containing a magnetic stir-bar was charged with $(S)$-S9 $(4.6 \mathrm{mg}, 16 \mu \mathrm{mol})$ which was taken up with DCM $(157 \mu \mathrm{L})$. The solution was cooled to $0{ }^{\circ} \mathrm{C}$ and DMAP (small crystal) and DCC (4.8 mg, $24 \mu \mathrm{mol}, 1.5$ equiv), and 3,5dinitrobenzoic acid ( $4.0 \mathrm{mg}, 19 \mu \mathrm{mol}, 1.2$ equiv) were added in that order. The reaction was allowed to warm to room temperature and stirred for 30 minutes. The reaction mixture filtered through a short plug of Celite eluting with DCM. Following concentration of the filtrate, the crude residue was purified via $\mathrm{SiO}_{2}$ column chromatography using hexanes:EtOAc (9:1 v:v) as the mobile phase. The title compound was obtained as a colorless solid $(5.1 \mathrm{mg}, 11 \mu \mathrm{mol}, 67 \%$ yield, $\left.\mathrm{MP}=135 \cdot 0-137.0^{\circ} \mathrm{C}\right)$. X-ray quality crystals of $(S)$-S10 were obtained by vapor diffusion using EtOAc (solvent) and pentanes (anti-solvent) at room temperature. ${ }^{1} \mathbf{H}$ NMR $(400 \mathrm{MHz}$, $\left.\mathrm{CDCl}_{3}\right) \delta 9.00(\mathrm{t}, J=2.2 \mathrm{~Hz}, 1 \mathrm{H}), 8.61(\mathrm{~d}, J=2.2 \mathrm{~Hz}, 2 \mathrm{H}), 7.97-7.92(\mathrm{~m}, 1 \mathrm{H}), 7.68(\mathrm{dd}, J=$ 8.7, $1.9 \mathrm{~Hz}, 2 \mathrm{H}), 7.58(\mathrm{~d}, J=8.8 \mathrm{~Hz}, 1 \mathrm{H}), 7.48(\mathrm{~d}, J=8.0 \mathrm{~Hz}, 1 \mathrm{H}), 7.32-7.19(\mathrm{~m}, 2 \mathrm{H}), 5.25$ $(\mathrm{t}, J=6.8 \mathrm{~Hz}, 1 \mathrm{H}), 4.75-4.65(\mathrm{~m}, 2 \mathrm{H}), 4.54(\mathrm{dd}, J=11.0,6.7 \mathrm{~Hz}, 1 \mathrm{H}), 4.18(\mathrm{~d}, J=11.5 \mathrm{~Hz}$, $1 \mathrm{H}), 1.84-1.72(\mathrm{~m}, 1 \mathrm{H}), 1.36(\mathrm{~s}, 3 \mathrm{H}), 1.25(\mathrm{~s}, 3 \mathrm{H}), 0.79-0.69(\mathrm{~m}, 2 \mathrm{H}), 0.65-0.55(\mathrm{~m}, 1 \mathrm{H})$, 
$0.13-0.00(\mathrm{~m}, 1 \mathrm{H}) .{ }^{13} \mathrm{C}$ NMR $\left(125 \mathrm{MHz}, \mathrm{CDCl}_{3}\right) \delta 209.2,162.0,148.1,141.6,133.2,132.6$, $131.8,128.8,128.8,128.7,128.1,126.8,125.8,125.6,125.5,121.9,90.1,77.5,71.9,52.1,42.4$, 22.5, 22.4, 16.0, 5.6, 0.7. IR (atr, $\left.\mathrm{cm}^{-1}\right) 3106,3014,2922,1952,1721,1631,1595,1551,1532$, 1456, 1329, 1341, 1273, 1162, 1078, 1023, 1000, 917, 862, 852, 823, 805, 767, 751, 729.

HRMS (ESI+, M+Na) Calc'd for $\mathrm{C}_{28} \mathrm{H}_{26} \mathrm{~N}_{2} \mathrm{NaO}_{6} 509.1683$, found 509.1682. $[\alpha]_{D}^{25}:+47.4(\mathrm{c}=$ $\left.0.11, \mathrm{CHCl}_{3}\right)$. 
Determination of absolute configuration of products obtained via the enantioselective Ircatalyzed allenylic alkylation: X-ray crystallographic analysis of $(R, S)-S 12$ :

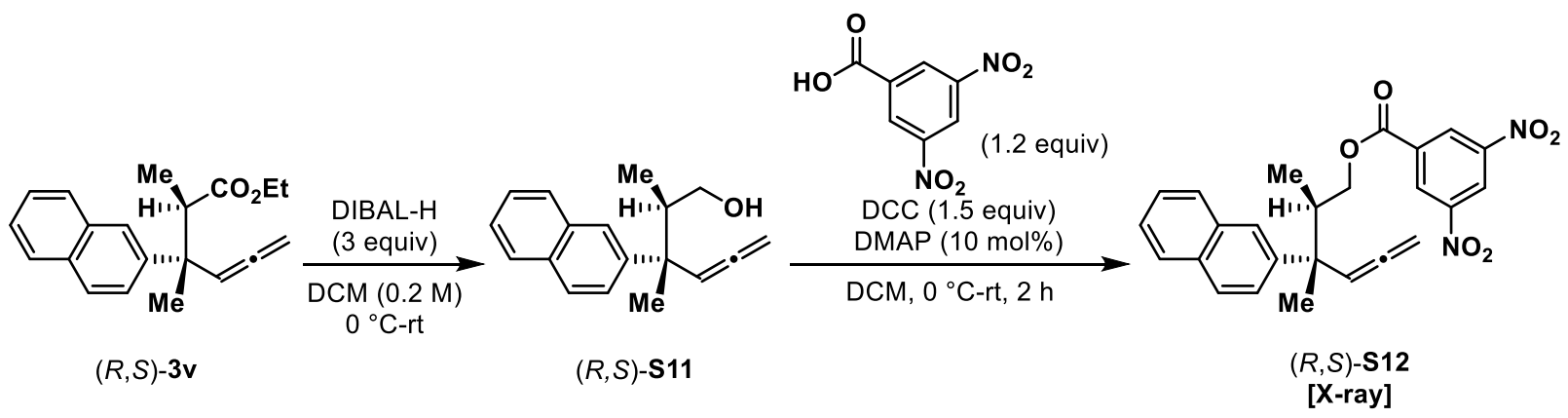

(2R,3S)-2,3-dimethyl-3-(naphthalen-2-yl)hexa-4,5-dien-1-ol [ $(R, S)-S 11]$ - DIBAL-H (122 $\mu \mathrm{L}, 0.122 \mathrm{mmol}, 3$ equiv, $1 \mathrm{M}$ in PhMe) was added dropwise to a DCM solution of $(R, S)-3 \mathbf{v}$ ( $12.0 \mathrm{mg}, 41 \mu \mathrm{mol}, 1$ equiv, $0.2 \mathrm{M}, 83 \%$ ee) at $0{ }^{\circ} \mathrm{C}$ and the resulting solution was warmed to room temperature and stirred for 2 hours. The solution was re-cooled to $0{ }^{\circ} \mathrm{C}$ where it was quenched with a saturated aqueous solution of Rochelle's salt. The resulting biphasic mixture was stirred vigorously for 30 minutes until clean phase separation as observed. The layers were separated, and the aqueous layer was extracted with DCM $(3 \times 5 \mathrm{~mL})$ and the combined organic layers were washed with brine, dried over $\mathrm{Na}_{2} \mathrm{SO}_{4}$, filtered and concentrated in vacuo. The crude residue was purified via $\mathrm{SiO}_{2}$ flash column chromatography using hexanes:EtOAc (3:1 $\mathrm{v}: \mathrm{v})$ and was isolated as a clear and colorless oil $(7.8 \mathrm{mg}, 0.031 \mathrm{mmol}, 76 \%$ yield $) .{ }^{1} \mathbf{H}$ NMR $\left(400 \mathrm{MHz}, \mathrm{CDCl}_{3}\right) \delta 7.83-7.79(\mathrm{~m}, 3 \mathrm{H}), 7.77(\mathrm{~d}, J=2.0 \mathrm{~Hz}, 1 \mathrm{H}), 7.55(\mathrm{dd}, J=8.7,2.0 \mathrm{~Hz}$, $1 \mathrm{H}), 7.51-7.42(\mathrm{~m}, 2 \mathrm{H}), 5.56(\mathrm{t}, J=6.7 \mathrm{~Hz}, 1 \mathrm{H}), 4.94-4.80(\mathrm{~m}, 2 \mathrm{H}), 3.78(\mathrm{dd}, J=10.6,4.0$ $\mathrm{Hz}, 1 \mathrm{H}), 3.39$ (dd, $J=10.6,8.1 \mathrm{~Hz}, 1 \mathrm{H}), 2.38-2.28(\mathrm{~m}, 1 \mathrm{H}), 1.44(\mathrm{~s}, 3 \mathrm{H}), 1.41(\mathrm{~s}(\mathrm{br}), 1 \mathrm{H})$, $0.91(\mathrm{~d}, J=6.8 \mathrm{~Hz}, 3 \mathrm{H}) .{ }^{13} \mathbf{C}$ NMR $\left(100 \mathrm{MHz}, \mathrm{CDCl}_{3}\right) \delta 207.8,145.0,133.3,131.9,128.0$, 127.9, 127.4, 126.0, 125.6, 124.9, 124.7, 97.6, 77.2, 65.7, 44.8, 44.2, 22.4, 12.9. IR (atr, $\left.\mathrm{cm}^{-1}\right)$ 3353, 3056, 2972, 2932, 1954, 1631, 1598, 1504, 1455, 1379, 1274, 1186, 1131, 1020, 948, 846, 817, 746. HRMS (ESI+, M+Na) Calc'd for $\mathrm{C}_{18} \mathrm{H}_{20} \mathrm{NaO} 275.1406$, found 275.1402. $[\alpha]_{D}^{23}$ $=-109.7\left(\mathrm{c}=0.39, \mathrm{CHCl}_{3}\right)$.

(2R,3S)-2,3-dimethyl-3-(naphthalen-2-yl)hexa-4,5-dien-1-yl $\quad 3,5$-dinitrobenzoate $[(R, S)$ S12] - A $1 \mathrm{~mL}$ screw-cap vial containing a magnetic stir-bar was charged with $(R, S)$-S11 (7.2 $\mathrm{mg}, 29 \mu \mathrm{mol})$ which was taken up with DCM $(285 \mu \mathrm{L})$. The solution was cooled to $0{ }^{\circ} \mathrm{C}$ and DMAP (0.35 mg. $2.9 \mu \mathrm{mol}, 10 \mathrm{~mol} \%$ ), 3,5-dinitrobenzoic acid (7.3 mg, $34 \mu \mathrm{mol}, 1.2$ equiv), and DCC ( $8.8 \mathrm{mg}, 43 \mu \mathrm{mol}, 1.5$ equiv), and were added in that order. The reaction was allowed to warm to room temperature and stirred for 2 hours. The reaction mixture filtered through a short plug of Celite eluting with DCM. Following concentration of the filtrate, the crude residue was purified via $\mathrm{SiO}_{2}$ column chromatography using hexanes:EtOAc $(9: 1 \mathrm{v}: \mathrm{v})$ as the mobile phase. The title compound was obtained as a colorless solid (11.4 mg, $26 \mu \mathrm{mol}, 89 \%$ yield, MP $\left.=138.0-139.2^{\circ} \mathrm{C}\right) . \mathrm{X}$-ray quality crystals of $(R, S)$-S12 were grown by slow evaporation of concentrated EtOAc solution of the benzoate at room temperature. ${ }^{1} \mathbf{H} \mathbf{~ N M R}\left(400 \mathrm{MHz}, \mathrm{CDCl}_{3}\right)$ $\delta 8.89(\mathrm{t}, J=2.2 \mathrm{~Hz}, 1 \mathrm{H}), 8.67(\mathrm{~d}, J=2.2 \mathrm{~Hz}, 2 \mathrm{H}), 7.72(\mathrm{~d}, J=2.0 \mathrm{~Hz}, 1 \mathrm{H}), 7.65-7.60(\mathrm{~m}$, 2H), $7.57-7.47(\mathrm{~m}, 2 \mathrm{H}), 7.35-7.26(\mathrm{~m}, 2 \mathrm{H}), 5.73(\mathrm{t}, J=6.7 \mathrm{~Hz}, 1 \mathrm{H}), 5.03-4.87(\mathrm{~m}, 2 \mathrm{H})$, $4.53(\mathrm{dd}, J=11.3,7.5 \mathrm{~Hz}, 1 \mathrm{H}), 4.32(\mathrm{dd}, J=11.3,6.6 \mathrm{~Hz}, 1 \mathrm{H}), 2.97-2.83(\mathrm{~m}, 1 \mathrm{H}), 1.45$ (s, $3 \mathrm{H}), 1.05(\mathrm{~d}, J=6.8 \mathrm{~Hz}, 3 \mathrm{H}) .{ }^{13} \mathbf{C}$ NMR $\left(100 \mathrm{MHz}, \mathrm{CDCl}_{3}\right) \delta 208.5,162.2,147.8,145.4,133.2$, $132.7,131.4,128.8,127.9,127.8,126.9,126.1,125.6,124.8,124.4,121.9,94.7,77.4,68.8$, 44.1, 40.7, 25.3, 13.00. IR (atr, $\left.\mathrm{cm}^{-1}\right)$ 3102, 2977, 2927, 1954, 1730, 1629, 1597, 1543, 14660, 1343, 1275, 1165, 1075, 976, 920, 854, 820, 730, 720. HRMS (ESI+, M+Na) Calc'd for $\mathrm{C}_{25} \mathrm{H}_{22} \mathrm{~N}_{2} \mathrm{NaO}_{6} 469.1370$, found 469.1367. [ $\left.\alpha\right]_{D}^{25}$ : -34.6 (c = 0.57, $\left.\mathrm{CHCl}_{3}\right)$. 
Determination of absolute configuration of products obtained via the enantioselective Ircatalyzed allenylic alkylation: $n O$ e experiment of $(R, S)-S 13$ :

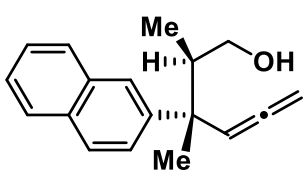

$(R, S)-\mathbf{S} 11$

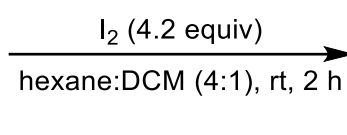

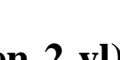

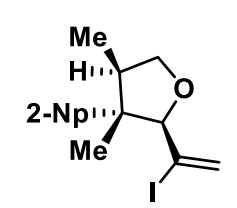

$(S, S, R)-S 13$

(2S,3S,4R)-3,4-dimethyl-3-(naphthalen-2-yl)-2-vinyltetrahydrofuran $[(S, S, R)$-S13 $]$ - A 25 $\mathrm{mL}$ round bottom flask containing a magnetic stir-bar was charged with $(R, S)-\mathbf{S 1 1}(15 \mathrm{mg}, 59$ $\mu \mathrm{mol})$ which was taken up with hexane $(2.0 \mathrm{~mL})$ and DCM $(0.5 \mathrm{~mL}) . \mathrm{I}_{2}(63.7 \mathrm{mg}, 0.25 \mathrm{mmol}$, 4.2 equiv) was added and the mixture was stirred at room temperature for 2 hours. The reaction mixture filtered through a short plug of Celite eluting with DCM. Following concentration of the filtrate, the crude residue was purified via $\mathrm{SiO}_{2}$ column chromatography using pentane:ether $(30: 1 \mathrm{v}: \mathrm{v})$ as the mobile phase. The title compound was obtained as a clear colorless oil (11.2 $\mathrm{mg}, 29 \mu \mathrm{mol}, 49 \%$ yield). ${ }^{1} \mathbf{H}$ NMR $\left(500 \mathrm{MHz}, \mathrm{CDCl}_{3}\right) \delta 7.91-7.78(\mathrm{~m}, 4 \mathrm{H}), 7.59(\mathrm{dd}, J=8.7$, $2.0 \mathrm{~Hz}, 1 \mathrm{H}), 7.56-7.44(\mathrm{~m}, 2 \mathrm{H}), 6.43(\mathrm{t}, J=1.4 \mathrm{~Hz}, 1 \mathrm{H}), 5.90(\mathrm{t}, J=1.2 \mathrm{~Hz}, 1 \mathrm{H}), 4.87(\mathrm{~d}, J=$ $1.6 \mathrm{~Hz}, 1 \mathrm{H}), 4.28(\mathrm{t}, J=8.2 \mathrm{~Hz}, 1 \mathrm{H}), 3.75(\mathrm{dd}, J=10.8,8.2 \mathrm{~Hz}, 1 \mathrm{H}), 2.94(\mathrm{ddq}, J=10.8,8.1$, $6.8 \mathrm{~Hz}, 1 \mathrm{H}), 1.32(\mathrm{~s}, 3 \mathrm{H}), 0.77(\mathrm{~d}, J=6.8 \mathrm{~Hz}, 3 \mathrm{H}) .{ }^{13} \mathbf{C ~ N M R}\left(126 \mathrm{MHz}, \mathrm{CDCl}_{3}\right) \delta 140.9,133.3$, $132.2,128.1,127.8,127.4,126.3,126.0,125.8,125.7,124.5,105.6,92.0,73.3,50.6,46.8,12.6$, 9.9. IR (atr, $\left.\mathrm{cm}^{-1}\right) 3055,2967,2925,2871,1726,1612,1506,1453,1385,1275,1132,1075$, 1041, 962, 897, 867, 846, 814, 741, 656, 476. HRMS (ESI+, M+Na) Calc'd for $\mathrm{C}_{18} \mathrm{H}_{19} \mathrm{NaOI}$ 401.0373, found 401.0371. $[\alpha]_{D}^{26}=-21.7\left(\mathrm{c}=0.40, \mathrm{CHCl}_{3}\right)$. 
nOe experiments
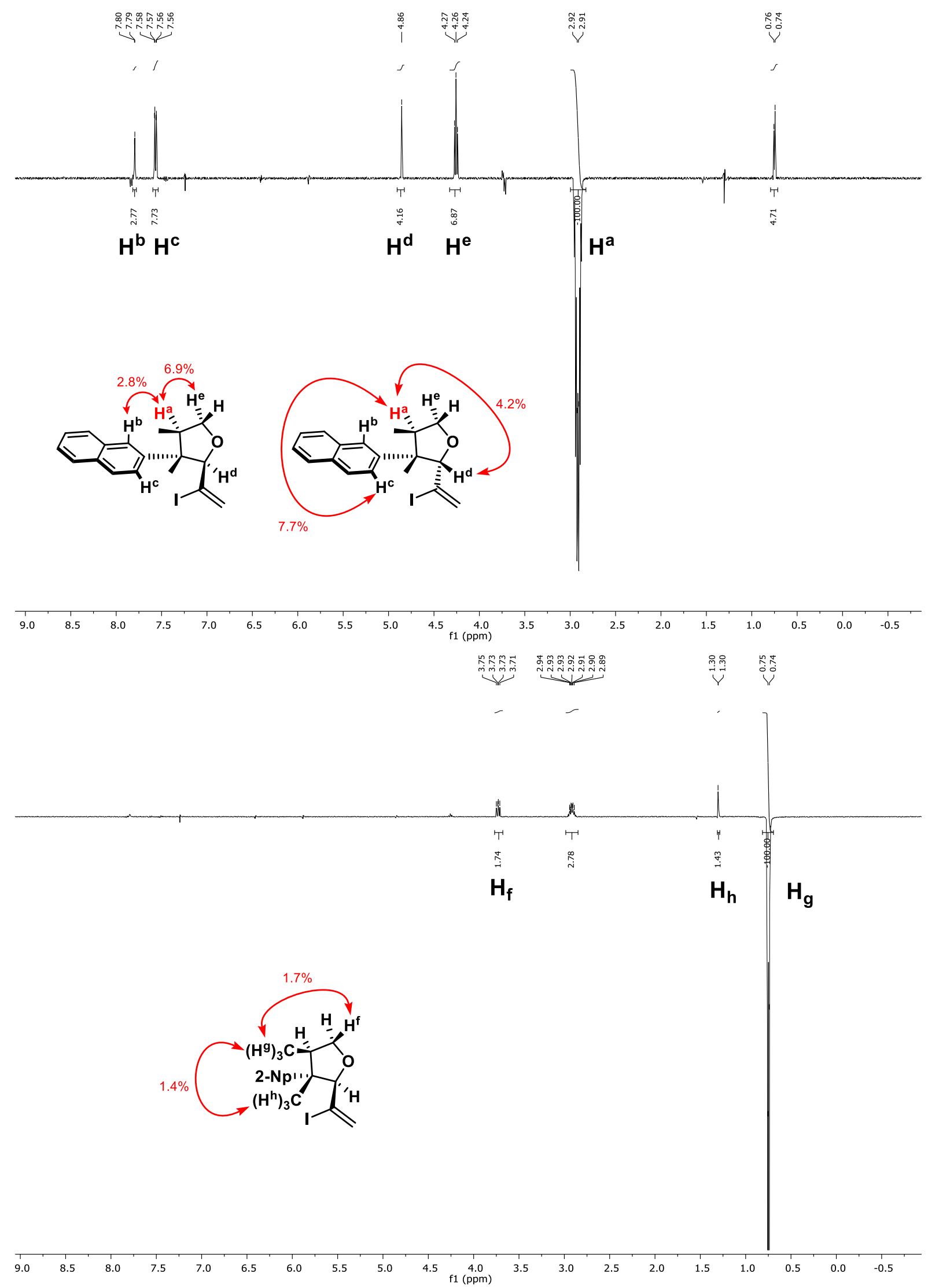


\section{X-Ray Crystallographic Data}

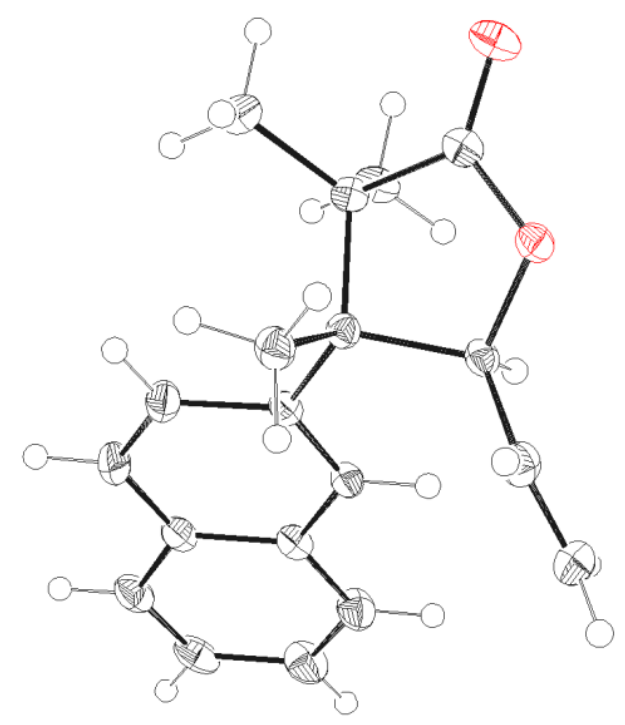

Figure S2. ORTEP diagram of $(R, S)-9$

Table 1 Crystal data and structure refinement for ca120819_1_1.

Identification code

ca120819_1_1

Empirical formula

$\mathrm{C}_{19} \mathrm{H}_{20} \mathrm{O}_{2}$

Formula weight

280.35

Temperature/K

100.0(1)

Crystal system

orthorhombic

Space group

$\mathrm{P} 2{ }_{1}{ }_{1} 2_{1}$

$\mathrm{a} / \AA$

$6.49950(10)$

$\mathrm{b} / \AA$

$9.33120(10)$

$\mathrm{c} / \AA$

25.1064(2)

$\alpha{ }^{\circ}$

90

$\beta /{ }^{\circ}$

90

$\gamma /{ }^{\circ}$

90

Volume $/ \AA^{3}$

1522.66(3)

Z

4

$\rho_{\text {calc }} \mathrm{g} / \mathrm{cm}^{3}$

1.223

$\mu / \mathrm{mm}^{-1}$

0.612

$\mathrm{F}(000)$

600.0

Crystal size $/ \mathrm{mm}^{3}$

$0.149 \times 0.1 \times 0.072$

Radiation

$\mathrm{CuK} \alpha(\lambda=1.54184)$

$2 \Theta$ range for data collection/ ${ }^{\circ} 7.042$ to 159.612

Index ranges

$-8 \leq \mathrm{h} \leq 8,-10 \leq \mathrm{k} \leq 11,-31 \leq 1 \leq 32$

Reflections collected

37649

Independent reflections

$3288\left[\mathrm{R}_{\text {int }}=0.0491, \mathrm{R}_{\text {sigma }}=0.0199\right]$

Data/restraints/parameters

$3288 / 0 / 193$

Goodness-of-fit on $\mathrm{F}^{2}$

1.053 
Final $\mathrm{R}$ indexes $[\mathrm{I}>=2 \sigma(\mathrm{I})] \quad \mathrm{R}_{1}=0.0308, \mathrm{wR}_{2}=0.0781$

Final $\mathrm{R}$ indexes [all data] $\quad \mathrm{R}_{1}=0.0321, \mathrm{wR}_{2}=0.0792$

Largest diff. peak/hole / e $\AA^{-3} \quad 0.20 /-0.17$

Flack parameter

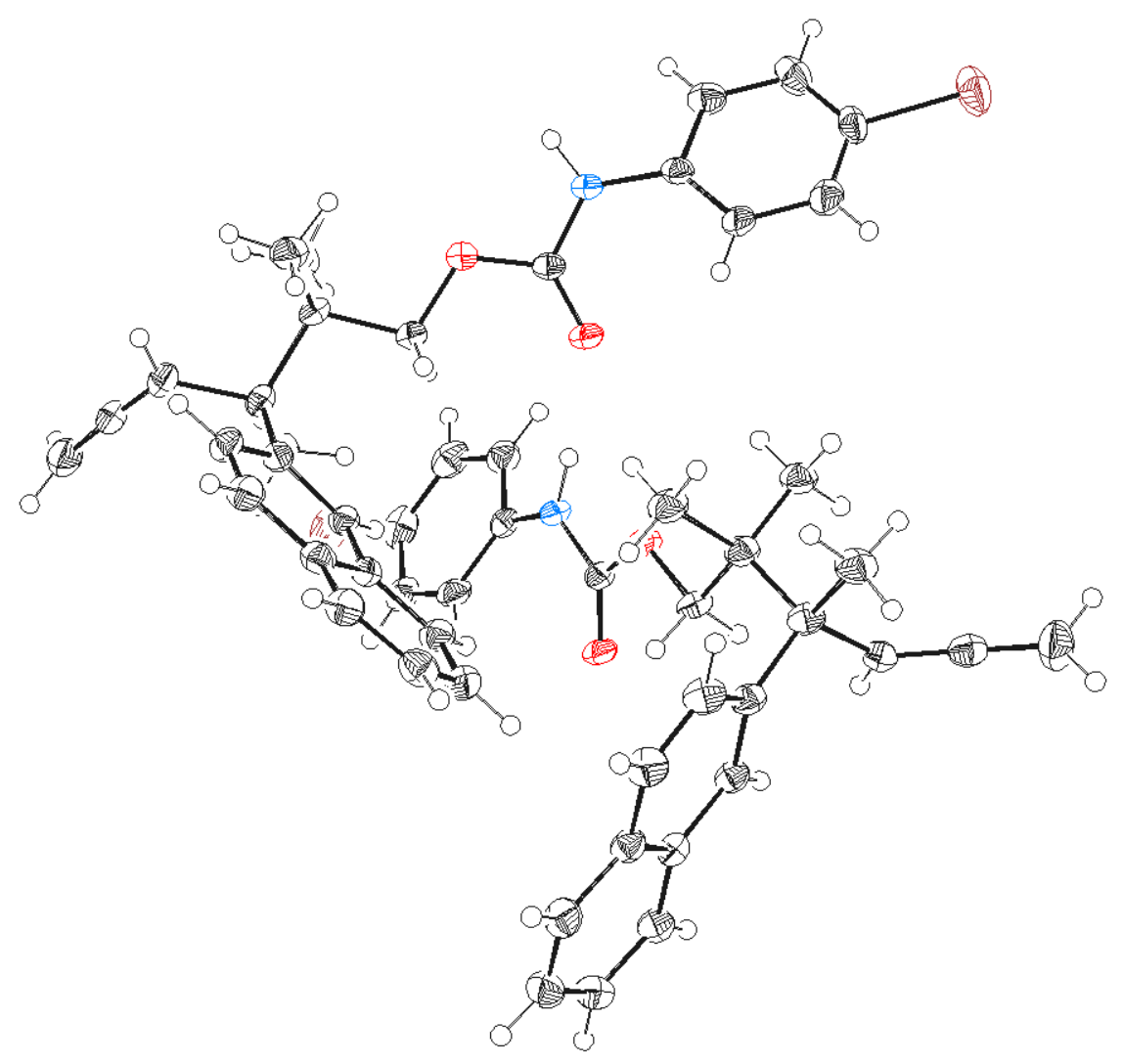

Figure S3. ORTEP diagram of $(R)$-S5

Table 2 Crystal data and structure refinement for ca120219_1_1.

Identification code

Empirical formula

Formula weight

Temperature/K

Crystal system

Space group

$\mathrm{a} / \AA$

$\mathrm{b} / \AA$

$\mathrm{c} / \AA$

$\alpha /{ }^{\circ}$

$\beta /{ }^{\circ}$

$\gamma /{ }^{\circ}$

Volume $/ \AA^{3}$ ca120219_1_1

$\mathrm{C}_{26} \mathrm{H}_{26} \mathrm{BrNO}_{2}$

464.39

$100.0(1)$

monoclinic

$\mathrm{P} 21$

9.6351(2)

25.6665(3)

$10.3770(2)$

90

116.625(3)

90

2294.10(9) 
Z

$\rho_{\text {calc }} / \mathrm{cm}^{3}$

1.345

$\mu / \mathrm{mm}^{-1}$

$\mathrm{F}(000)$

Crystal size $/ \mathrm{mm}^{3}$

Radiation

$2 \Theta$ range for data collection $/{ }^{\circ} 6.888$ to 160.2

Index ranges

Reflections collected

Independent reflections

Data/restraints/parameters

Goodness-of-fit on $\mathrm{F}^{2}$

Final $R$ indexes $[\mathrm{I}>=2 \sigma(\mathrm{I})]$

Final $\mathrm{R}$ indexes [all data]

Largest diff. peak/hole / e $\AA^{-3} 0.57 /-0.96$

Flack parameter

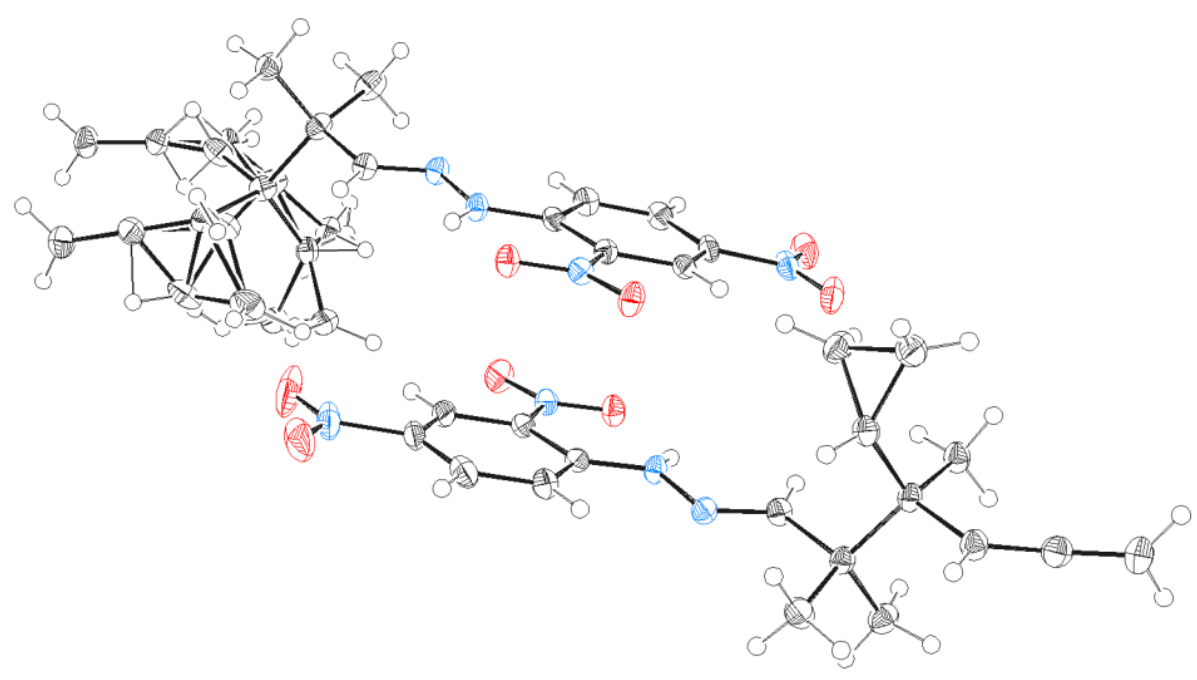

Figure S4. ORTEP diagram of $(R)$-S8

Table 3 Crystal data and structure refinement for ca150819_1_1.

Identification code

Empirical formula

Formula weight

Temperature/K

Crystal system

Space group

$\mathrm{a} / \AA$

$\mathrm{b} / \AA$

c/Å ca150819_1_1

$\mathrm{C}_{18} \mathrm{H}_{22} \mathrm{~N}_{4} \mathrm{O}_{4}$

358.39

100.0(1)

monoclinic

$\mathrm{P} 21$

6.66510(10)

18.9363(2)

14.1449(2) 


\begin{tabular}{|c|c|}
\hline$\alpha /^{\circ}$ & 90 \\
\hline$\beta /{ }^{\circ}$ & $91.1350(10)$ \\
\hline$\gamma /{ }^{\circ}$ & 90 \\
\hline Volume $/ \AA^{3}$ & $1784.91(4)$ \\
\hline $\mathrm{Z}$ & 4 \\
\hline$\rho_{\text {calc }} \mathrm{g} / \mathrm{cm}^{3}$ & 1.334 \\
\hline$\mu / \mathrm{mm}^{-1}$ & 0.793 \\
\hline $\mathrm{F}(000)$ & 760.0 \\
\hline Crystal size $/ \mathrm{mm}^{3}$ & $0.133 \times 0.025 \times 0.022$ \\
\hline Radiation & $\mathrm{CuK} \alpha(\lambda=1.54184)$ \\
\hline $2 \Theta$ range for data collection $/^{\circ}$ & 6.25 to 159.368 \\
\hline Index ranges & $-8 \leq \mathrm{h} \leq 8,-23 \leq \mathrm{k} \leq 23,-17 \leq 1 \leq 15$ \\
\hline Reflections collected & 22607 \\
\hline Independent reflections & $7401\left[\mathrm{R}_{\mathrm{int}}=0.0318, \mathrm{R}_{\text {sigma }}=0.0341\right]$ \\
\hline Data/restraints/parameters & $7401 / 209 / 567$ \\
\hline Goodness-of-fit on $\mathrm{F}^{2}$ & 1.061 \\
\hline Final $R$ indexes $[\mathrm{I}>=2 \sigma(\mathrm{I})]$ & $\mathrm{R}_{1}=0.0355, \mathrm{wR}_{2}=0.0913$ \\
\hline Final $\mathrm{R}$ indexes [all data] & $\mathrm{R}_{1}=0.0404, \mathrm{wR}_{2}=0.0942$ \\
\hline Largest diff. peak/hole / e $\AA^{-3}$ & $0.19 /-0.21$ \\
\hline Flack parameter & $-0.01(10)$ \\
\hline
\end{tabular}

NOTE: Quaternary carbon in one structure is disordered by rotation.

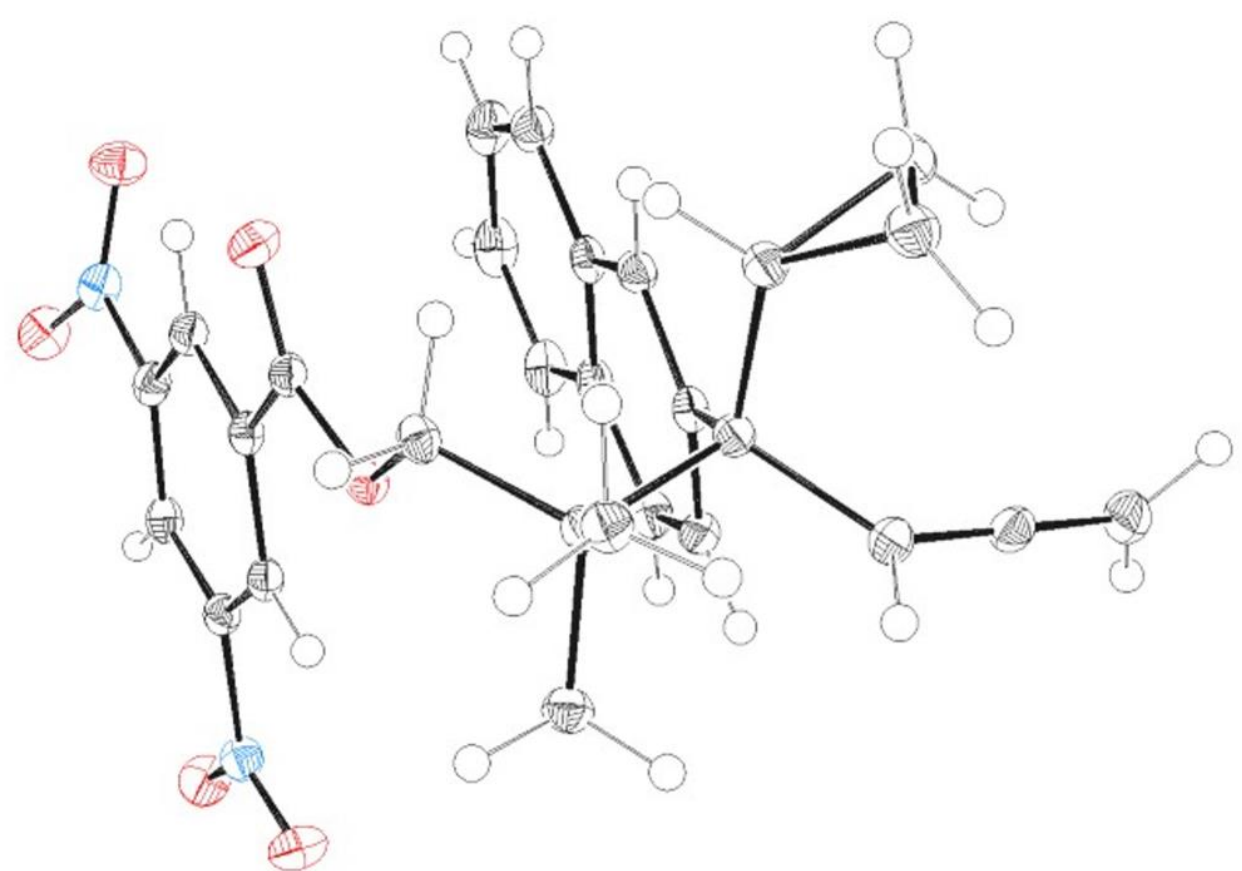

Figure S5. ORTEP diagram of $(S)$-S10

Table 4 Crystal data and structure refinement for ca060619_2_1.

Identification code ca060619_2_1 


\begin{tabular}{|c|c|}
\hline Empirical formula & $\mathrm{C}_{28} \mathrm{H}_{26} \mathrm{~N}_{2} \mathrm{O}_{6}$ \\
\hline Formula weight & 486.51 \\
\hline Temperature/K & $100.0(1)$ \\
\hline Crystal system & orthorhombic \\
\hline Space group & $\mathrm{P} 2{ }_{1} 2{ }_{1}{ }_{1}$ \\
\hline $\mathrm{a} / \AA ̊$ & $7.20532(5)$ \\
\hline $\mathrm{b} / \AA$ & $8.01606(5)$ \\
\hline $\mathrm{c} / \AA$ & $40.3538(2)$ \\
\hline$\alpha /^{\circ}$ & 90 \\
\hline$\beta /{ }^{\circ}$ & 90 \\
\hline$\gamma /{ }^{\circ}$ & 90 \\
\hline Volume $/ \AA^{3}$ & $2330.77(2)$ \\
\hline $\mathrm{Z}$ & 4 \\
\hline$\rho_{\text {calc }} g / \mathrm{cm}^{3}$ & 1.386 \\
\hline$\mu / \mathrm{mm}^{-1}$ & 0.807 \\
\hline $\mathrm{F}(000)$ & 1024.0 \\
\hline Crystal size $/ \mathrm{mm}^{3}$ & $0.167 \times 0.111 \times 0.048$ \\
\hline Radiation & $\operatorname{CuK} \alpha(\lambda=1.54184)$ \\
\hline $2 \Theta$ range for data collection ${ }^{\circ}$ & 4.38 to 159.444 \\
\hline Index ranges & $-9 \leq \mathrm{h} \leq 7,-10 \leq \mathrm{k} \leq 10,-51 \leq 1 \leq 50$ \\
\hline Reflections collected & 61302 \\
\hline Independent reflections & $4983\left[\mathrm{R}_{\mathrm{int}}=0.0445, \mathrm{R}_{\mathrm{sigma}}=0.0175\right]$ \\
\hline Data/restraints/parameters & $4983 / 3 / 333$ \\
\hline Goodness-of-fit on $\mathrm{F}^{2}$ & 1.067 \\
\hline Final $R$ indexes $[\mathrm{I}>=2 \sigma(\mathrm{I})]$ & $\mathrm{R}_{1}=0.0270, \mathrm{wR}_{2}=0.0678$ \\
\hline Final $\mathrm{R}$ indexes [all data] & $\mathrm{R}_{1}=0.0278, \mathrm{wR}_{2}=0.0684$ \\
\hline Largest diff. peak/hole / e $\AA^{-3}$ & $0.19 /-0.21$ \\
\hline Flack parameter & $0.06(5)$ \\
\hline
\end{tabular}




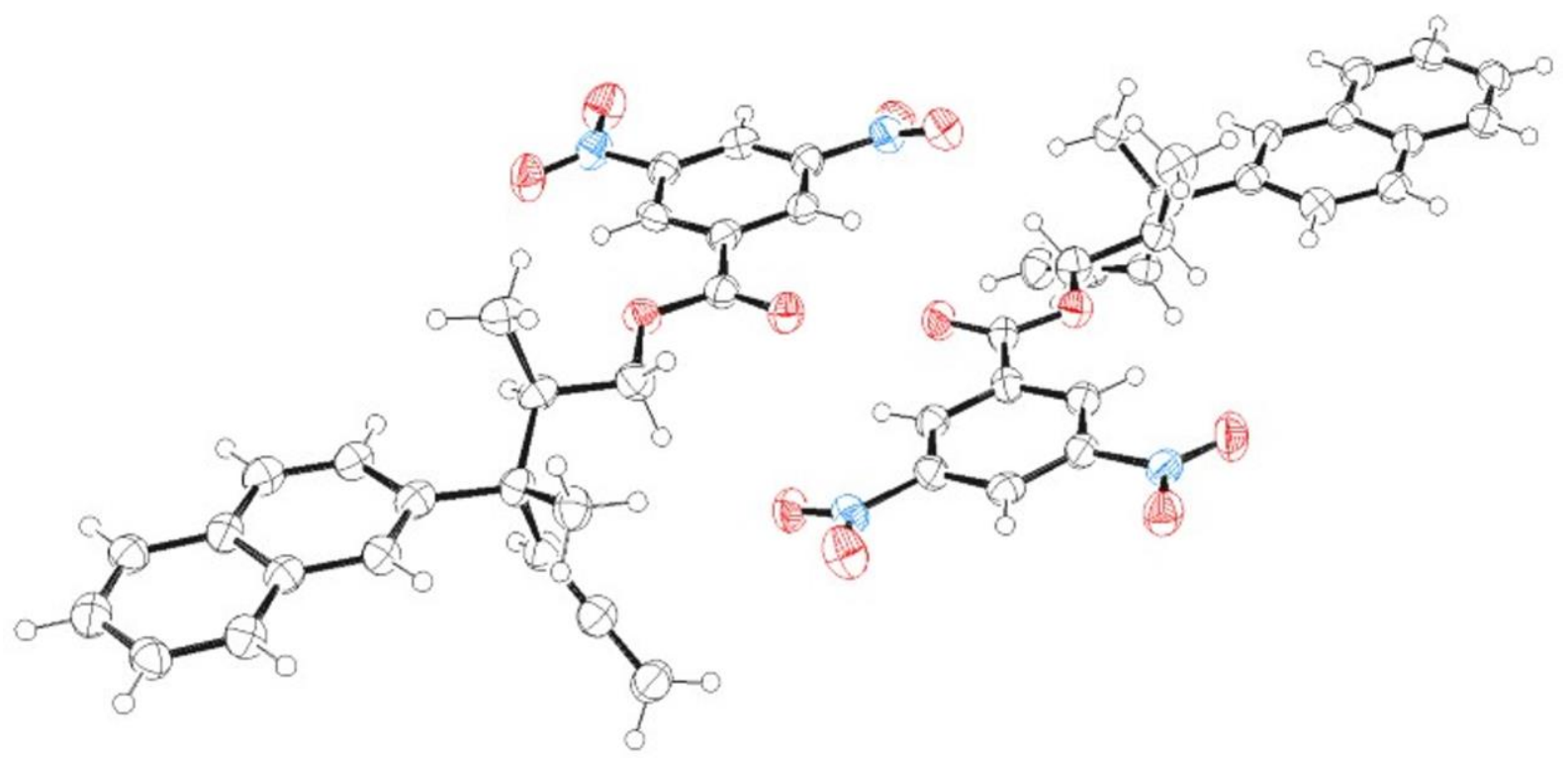

Figure S6. ORTEP diagram of $(R, S)-\mathrm{S12}$

Table 5 Crystal data and structure refinement for ca090919_4_1.

Identification code

ca090919_4_1

Empirical formula

$\mathrm{C}_{25} \mathrm{H}_{22} \mathrm{~N}_{2} \mathrm{O}_{6}$

Formula weight

446.44

Temperature/K

$100.0(1)$

Crystal system

monoclinic

Space group

$\mathrm{P} 21$

$\mathrm{a} / \AA$

7.0168(7)

$\mathrm{b} / \AA$

21.420(3)

$\mathrm{c} / \AA$

$14.7332(13)$

$\alpha /{ }^{\circ}$

90

$\beta /{ }^{\circ}$

$99.068(8)$

$\gamma /{ }^{\circ}$

90

Volume $/ \AA^{3}$

2186.7(4)

$\mathrm{Z}$

4

$\rho_{\text {calc }} \mathrm{g} / \mathrm{cm}^{3}$

1.356

$\mu / \mathrm{mm}^{-1}$

0.811

$\mathrm{F}(000)$

936.0

Crystal size $/ \mathrm{mm}^{3}$

$0.061 \times 0.049 \times 0.017$

Radiation

$\mathrm{Cu} \mathrm{K \alpha}(\lambda=1.54184)$

$2 \Theta$ range for data collection $/^{\circ}$

Index ranges

6.074 to 160.794

Reflections collected

$-8 \leq \mathrm{h} \leq 8,-27 \leq \mathrm{k} \leq 27,-3 \leq 1 \leq 18$

Independent reflections

8823

$8823\left[\mathrm{R}_{\text {int }}=0.1131, \mathrm{R}_{\text {sigma }}=0.1044\right]$

Data/restraints/parameters

$8823 / 9 / 612$

Goodness-of-fit on $\mathrm{F}^{2}$

1.030 
Final $R$ indexes $[\mathrm{I}>=2 \sigma(\mathrm{I})] \quad \mathrm{R}_{1}=0.0685, \mathrm{wR}_{2}=0.1622$

Final $\mathrm{R}$ indexes [all data] $\quad \mathrm{R}_{1}=0.1351, \mathrm{wR}_{2}=0.2069$

Largest diff. peak/hole / e $\AA^{-3} \quad 0.26 /-0.33$

Flack parameter $\quad-0.1(3)$ 


\section{Mechanistic Studies}

Partial conversion experiment - testing for a kinetic resolution:

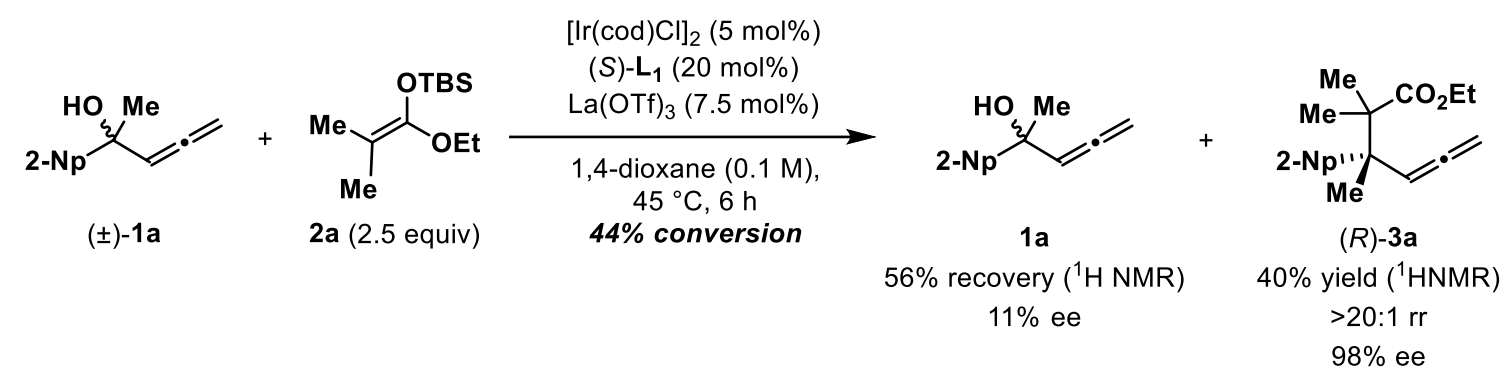

Reaction was carried out according to general procedure 2a using ( \pm )-1a (84 mg, $0.4 \mathrm{mmol}, 1$ equiv), $\left[\operatorname{Ir}(\operatorname{cod}) \mathrm{Cl}_{2}(13.4 \mathrm{mg}, 20 \mu \mathrm{mol}, 5.0 \mathrm{~mol} \%),(S)-\mathbf{L} \mathbf{1}(40.6 \mathrm{mg}, 80 \mu \mathrm{mol}, 20 \mathrm{~mol} \%), \mathbf{2 a}\right.$ $\left(246 \mu \mathrm{L}, 1.0 \mathrm{mmol}, 2.5\right.$ equiv), and $\mathrm{La}(\mathrm{OTf})_{3}(17.6 \mathrm{mg}, 30 \mu \mathrm{mol}, 7.5 \mathrm{~mol} \%)$ in 1,4-dioxane (4.0 $\mathrm{mL}$ ) at $45^{\circ} \mathrm{C}$ for 6 hours. The reaction mixture was filtered through a short plug of $\mathrm{SiO}_{2}$ eluting with $\mathrm{Et}_{2} \mathrm{O}$. After removal of all volatiles in vacuo, the extent of conversion of $( \pm)-\mathbf{1 a}$, the yield of $(R)-3 \mathbf{a}$, and regioselectivity (rr) were determined by quantitative 1HNMR of the residue using tetrachloroethane as an internal standard. Samples of remaining starting material 1a and product $(R)$-3a for chiral chromatographic analysis were isolated by preparative TLC. Enantiomeric purity of recovered 1a and $(R)$-3a were determined by HPLC analysis in comparison with racemic material. Enantiomeric purity of recovered 1a was determined by HPLC analysis in comparison with racemic material $\mathrm{T}_{\mathrm{R}}=10.7$ (major), $\mathrm{T}_{\mathrm{R}}=12.1$ (minor) (55.5:44.5 er shown); Chiracel IC column; 98\% hexane and 2\% iPrOH $1.0 \mathrm{~mL} / \mathrm{min}, 220.0 \mathrm{~nm}$, $\left.480 \mathrm{psi}, 25^{\circ} \mathrm{C}\right)$. Enantiomeric purity of $(R)$-3a was determined by HPLC analysis in comparison with racemic material $\mathrm{T}_{\mathrm{R}}=5.6$ (minor) $\mathrm{T}_{\mathrm{R}}=6.4$ (major) (99:1 er shown); Chiracel IC column; $98 \%$ hexane and $2 \% \mathrm{iPrOH} 1.0 \mathrm{~mL} / \mathrm{min}, 220.0 \mathrm{~nm}, 480 \mathrm{psi}, 25^{\circ} \mathrm{C}$ ). All spectral data of recovered 1a and $(R)-\mathbf{3 a}$ were identical to those reported above.
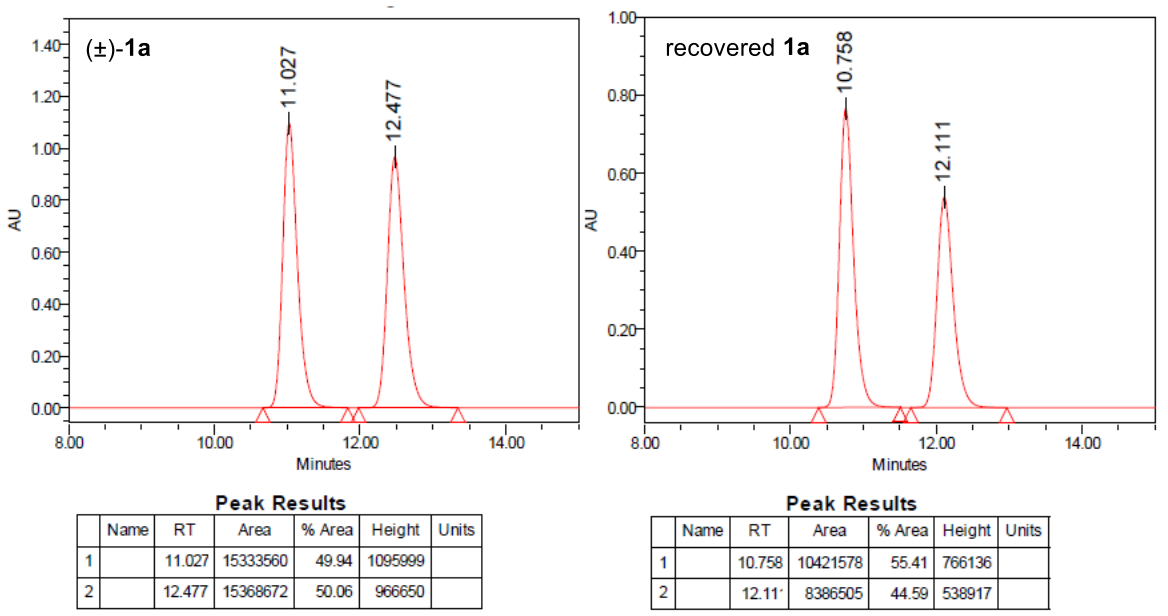

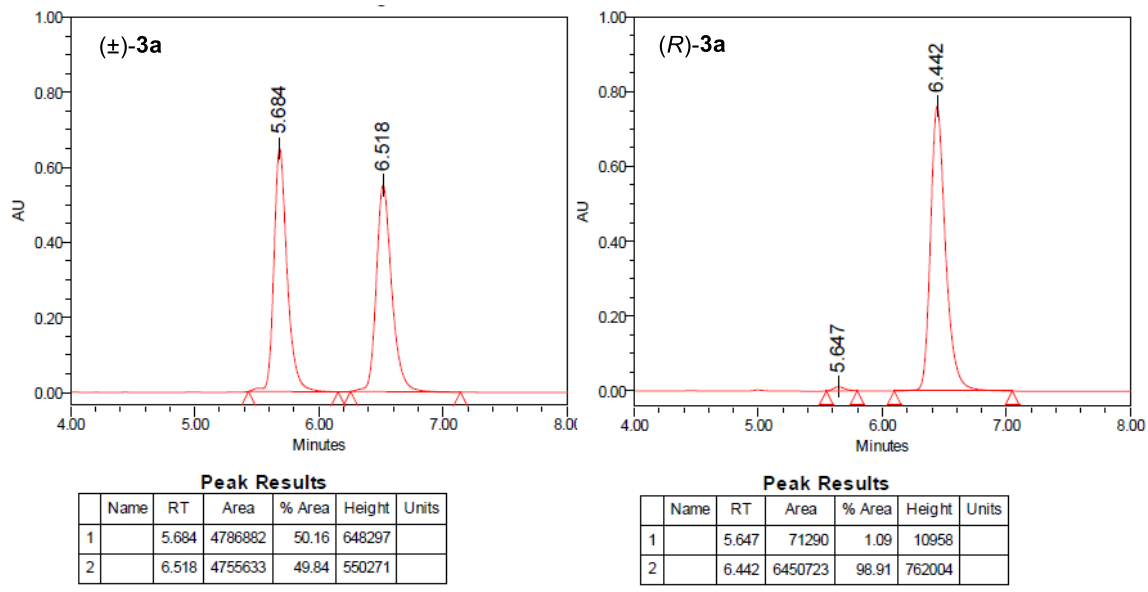

Reaction of enantioenriched tertiary alcohol with (R)- and (S)-L1:

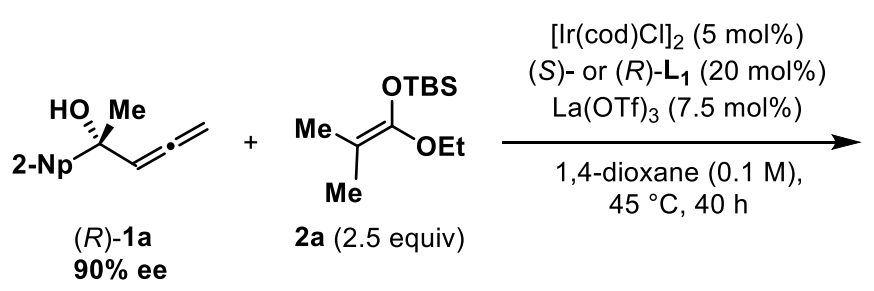

$(S)-L_{1}:$

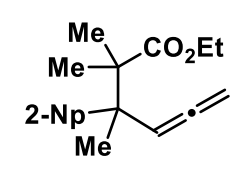

$(R)$ - or $(S)-3 \mathbf{a}$

(R)-3a: $75 \%$ yield $>20: 1 \mathrm{rr} ; 99 \%$ ee

(S)-3a: $81 \%$ yield
$>20: 1 \mathrm{rr} 96 \%$ ee

Reaction was carried out according to general procedure 2 a using $(R)-\mathbf{1 a}^{1}(84 \mathrm{mg}, 0.4 \mathrm{mmol}, 1$ equiv), $[\operatorname{Ir}(\operatorname{cod}) \mathrm{Cl}]_{2}(13.4 \mathrm{mg}, 20 \mu \mathrm{mol}, 5.0 \mathrm{~mol} \%)$, $(S)$ - or $(R)-\mathbf{L} 1$ (40.6 mg, $80 \mu \mathrm{mol}, 20$ mol\%), 2a (246 $\mu \mathrm{L}, 1.0 \mathrm{mmol}, 2.5$ equiv), and $\mathrm{La}(\mathrm{OTf})_{3}(17.6 \mathrm{mg}, 30 \mu \mathrm{mol}, 7.5 \mathrm{~mol} \%)$ in 1,4dioxane $(4.0 \mathrm{~mL})$ at $45^{\circ} \mathrm{C}$ for 40 hours. The reaction mixture was filtered through a short plug of $\mathrm{SiO}_{2}$ eluting with $\mathrm{Et}_{2} \mathrm{O}$. After removal of all volatiles in vacuo, the yield of $\mathbf{3 a}$, and regioselectivity ( $r r$ ) were determined by quantitative ${ }^{1} \mathrm{HNMR}$ of the residue using tetrachloroethane as an internal standard. Product 3a for chiral chromatographic analysis was isolated by preparative TLC. Enantiomeric purity of 3a were determined by HPLC analysis in comparison with racemic material. $\mathrm{T}_{\mathrm{R}}=5.6, \mathrm{~T}_{\mathrm{R}}=6.4\left(0.5: 99.5 \mathrm{er}\right.$ for reaction with $(S)-\mathbf{L}_{1}, 98: 2$ er for reaction with $(R)-\mathbf{L} 1)$; Chiracel IC column; 98\% hexane and $2 \% \mathrm{iPrOH} 1.0 \mathrm{~mL} / \mathrm{min}, 220.0$ $\mathrm{nm}, 480 \mathrm{psi}, 25^{\circ} \mathrm{C}$ ). All spectral data of recovered 3a was identical to those reported above. 
Reaction with $(S)-\mathbf{L}_{\mathbf{1}}$
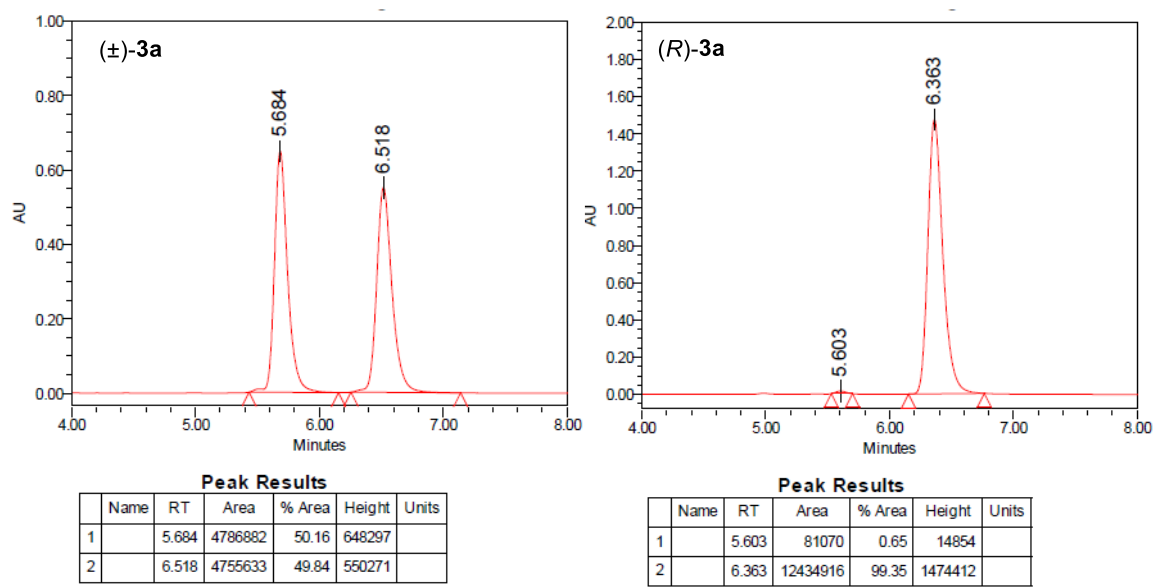

Reaction with $(R)-\mathbf{L} \mathbf{1}$
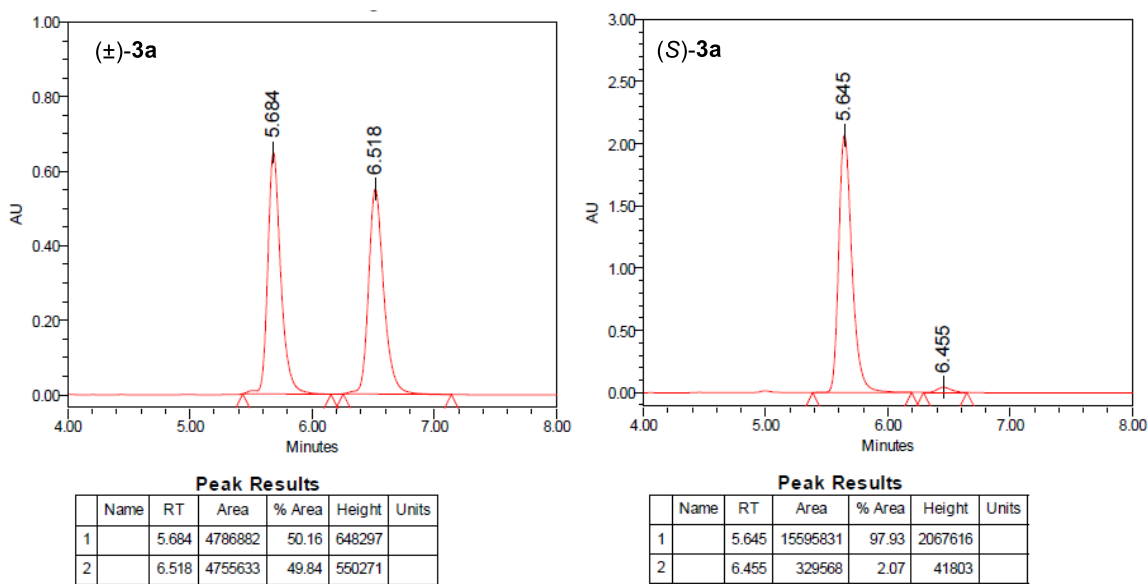


\section{NMR Spectra}
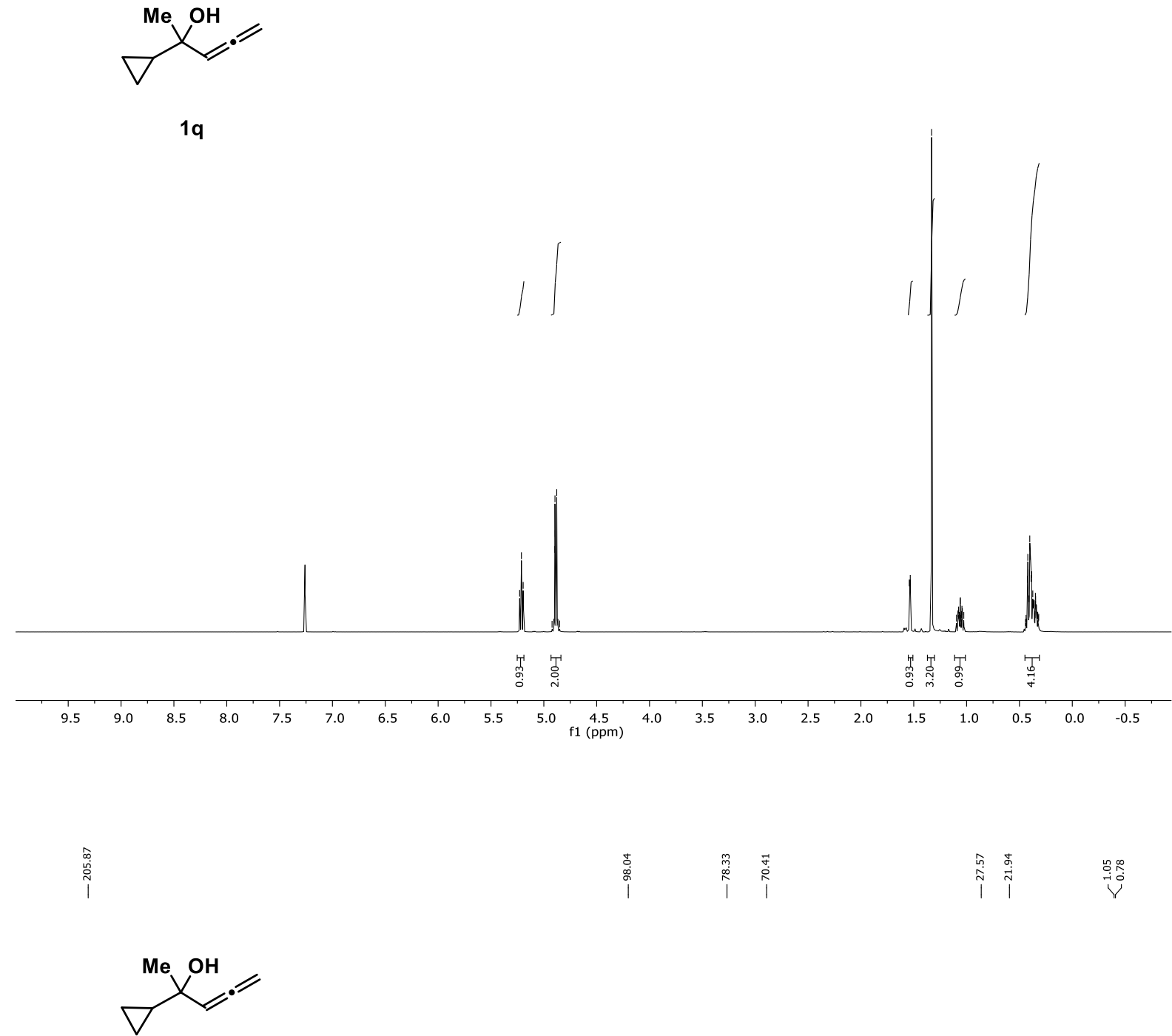

19

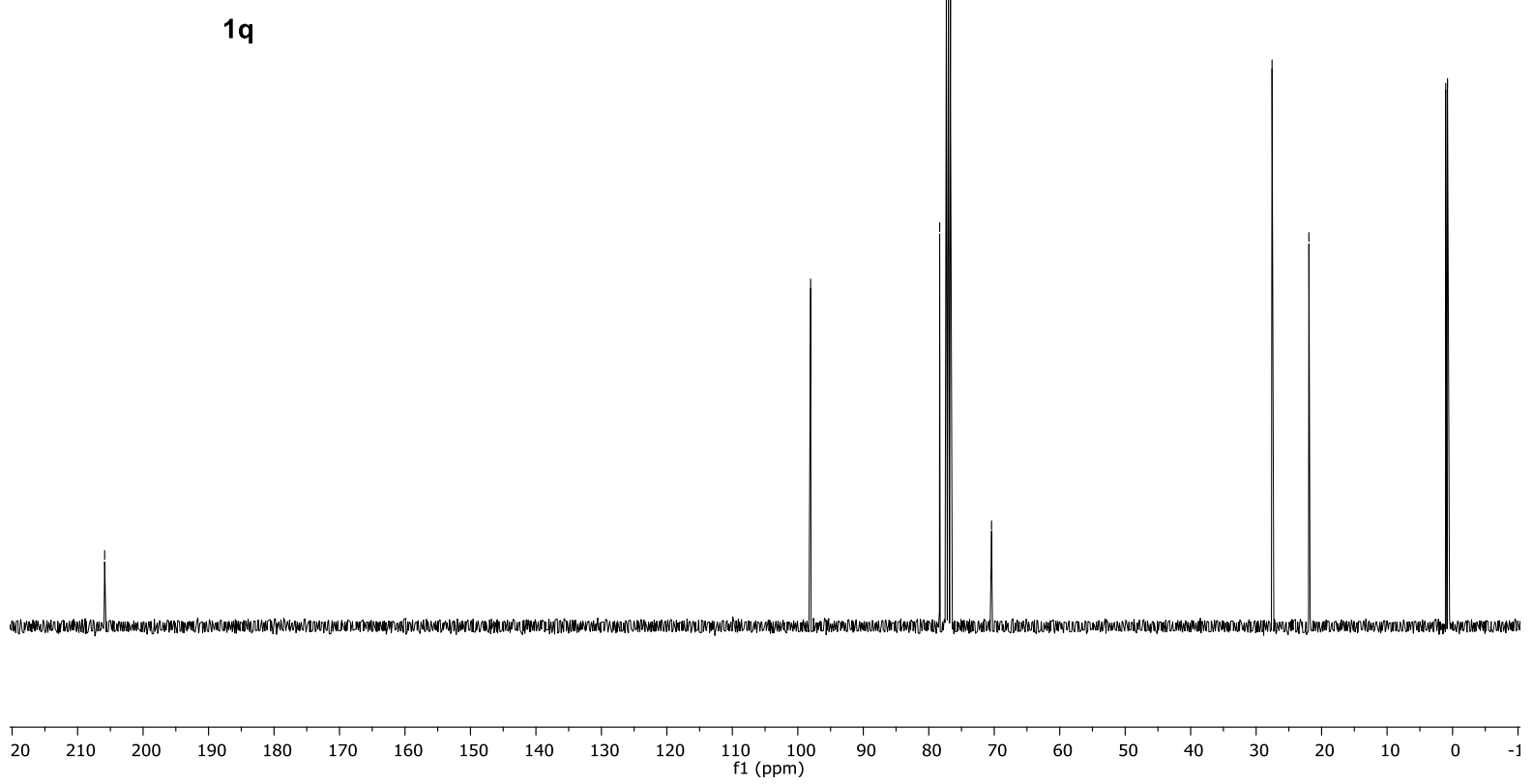



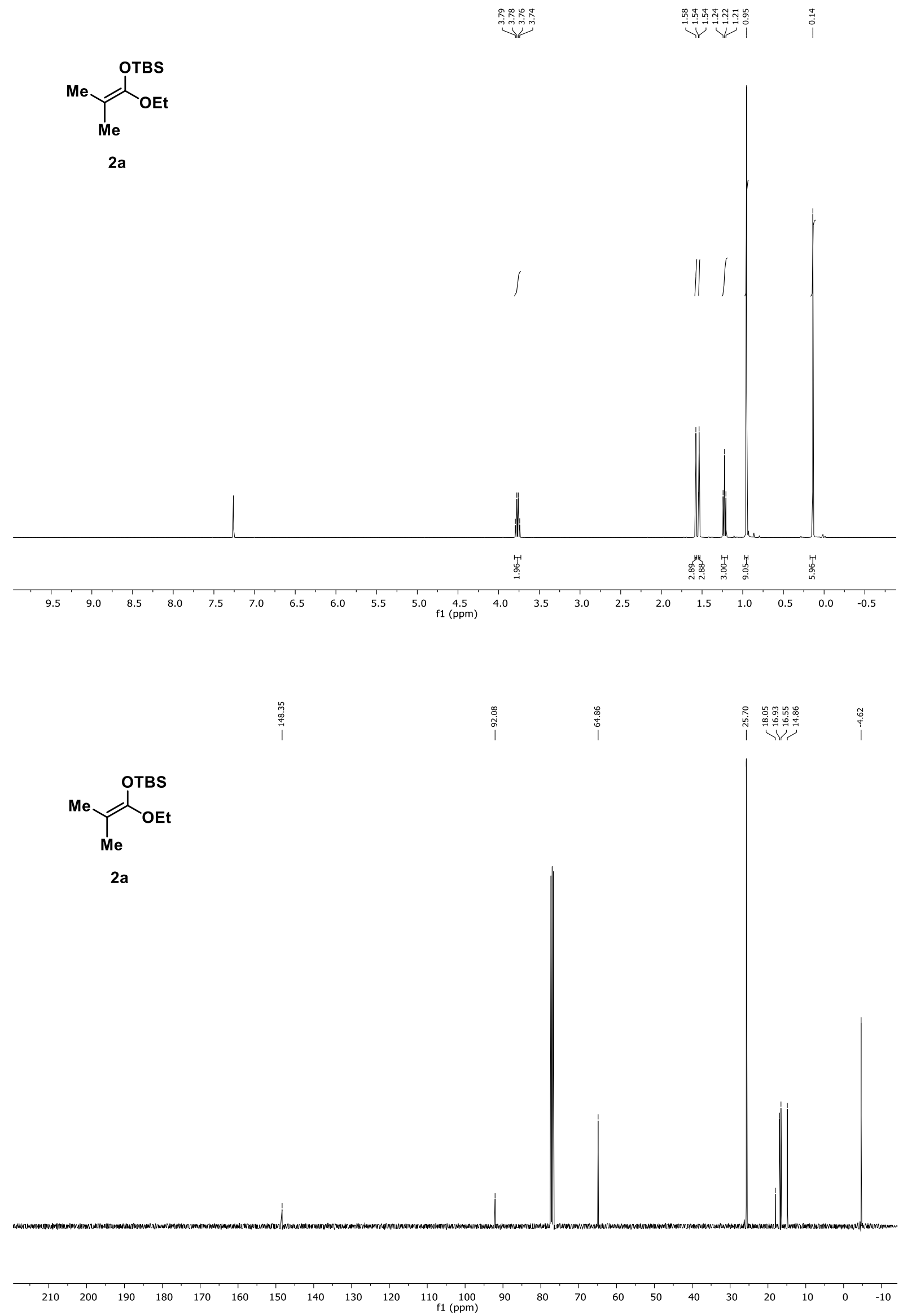


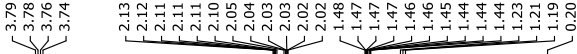

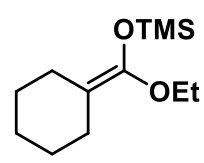

$2 g$

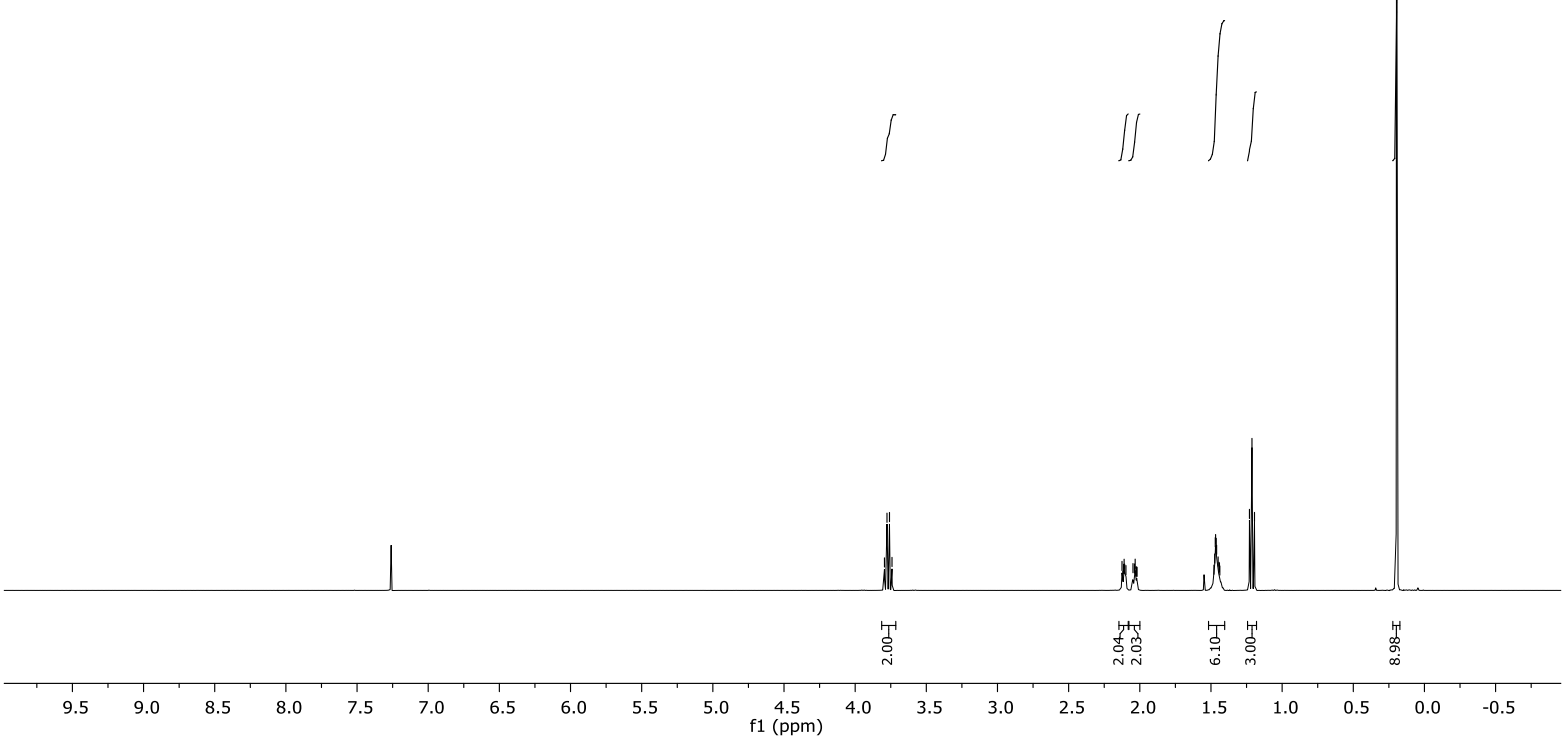

品

?

苦

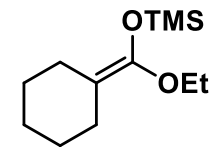

$2 \mathrm{~g}$

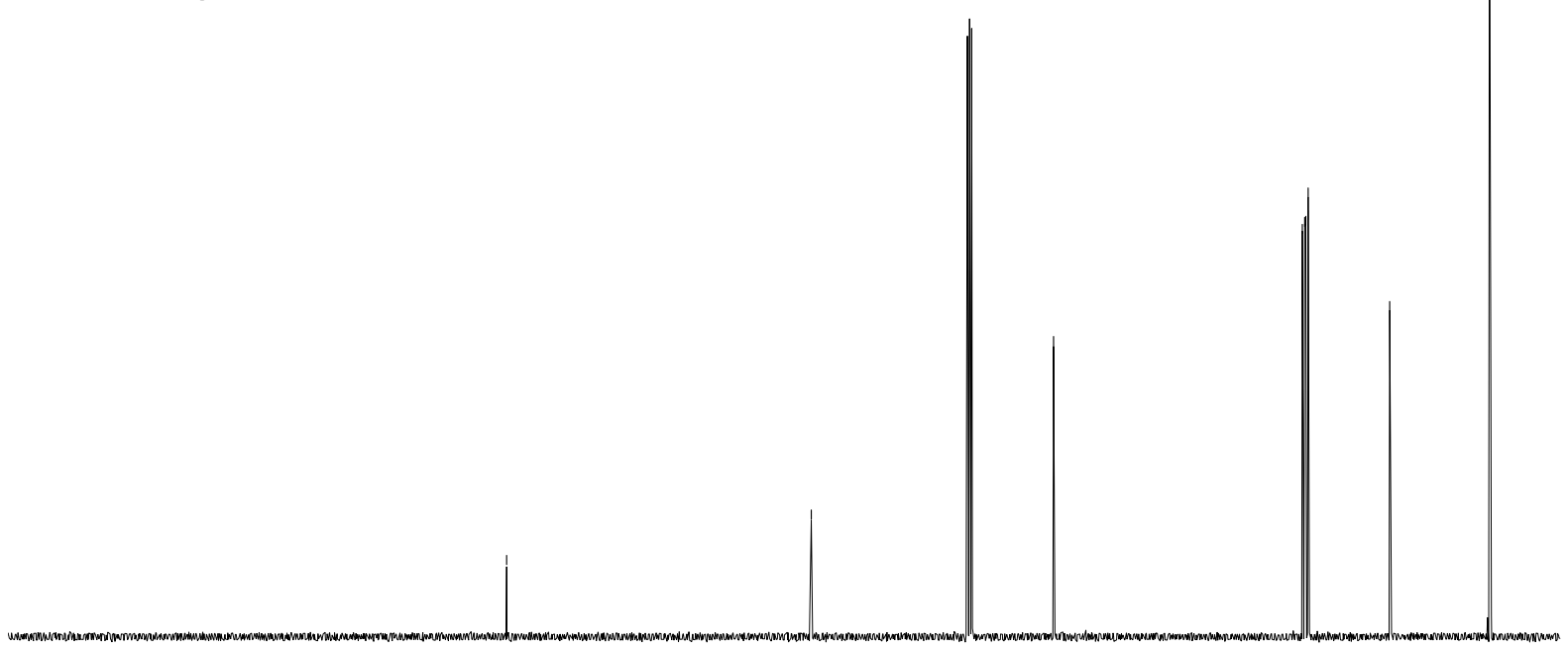


<smiles>CCOC(OC)=C1CCC(F)(F)CC1</smiles>

$2 \mathrm{~h}$

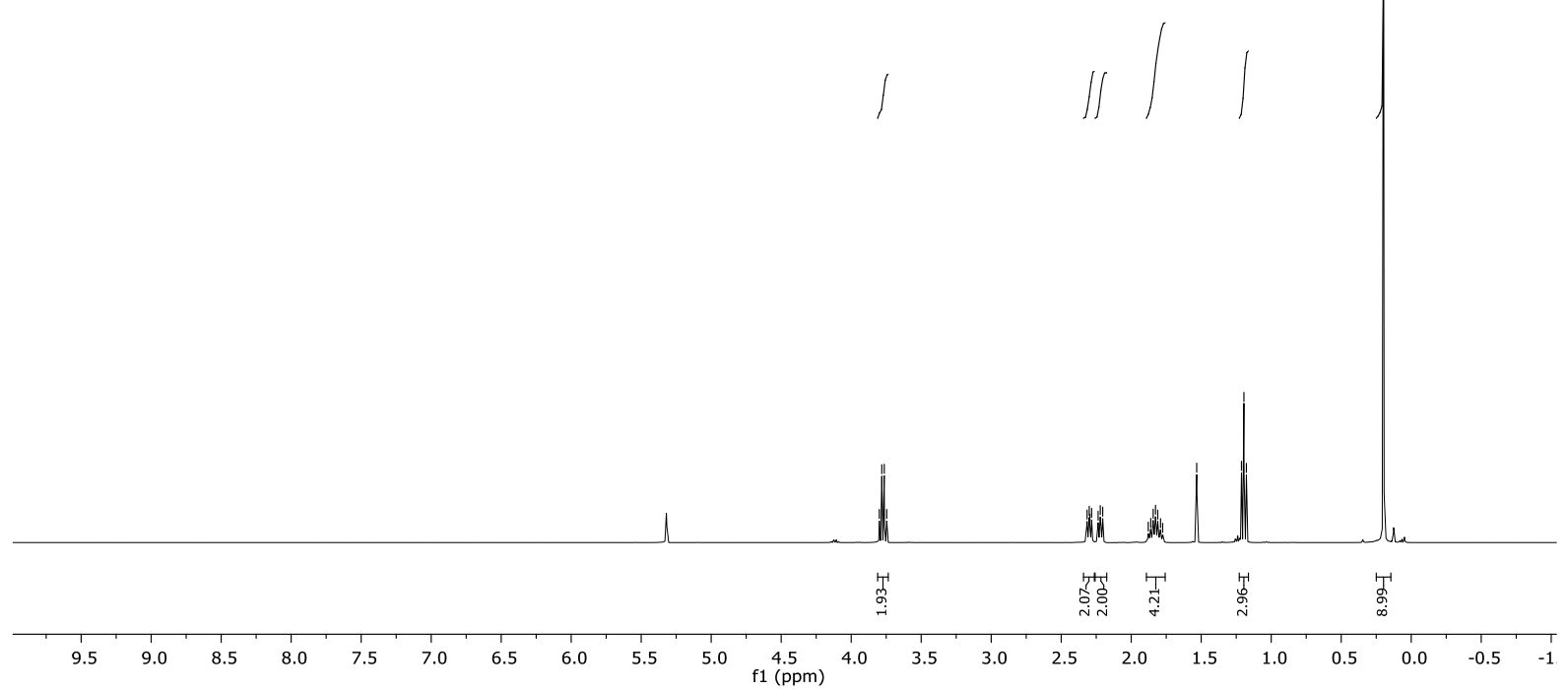<smiles>CCOC(OC)=C1CCC(F)(F)CC1</smiles>

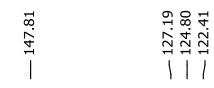

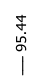

$\underset{\substack{7 \\ \text { i }}}{1}$

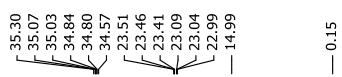

$2 \mathrm{~h}$

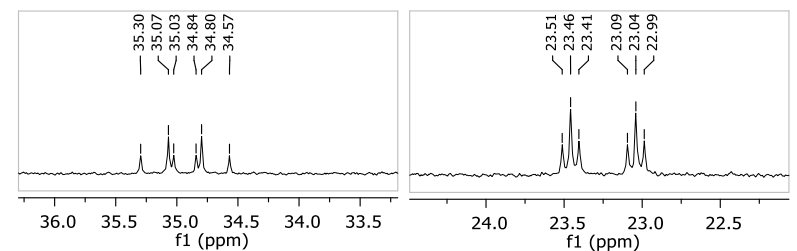

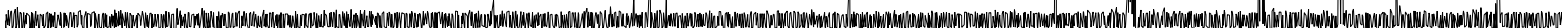

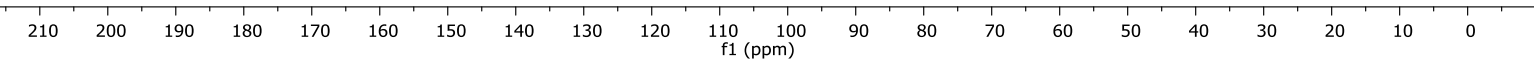



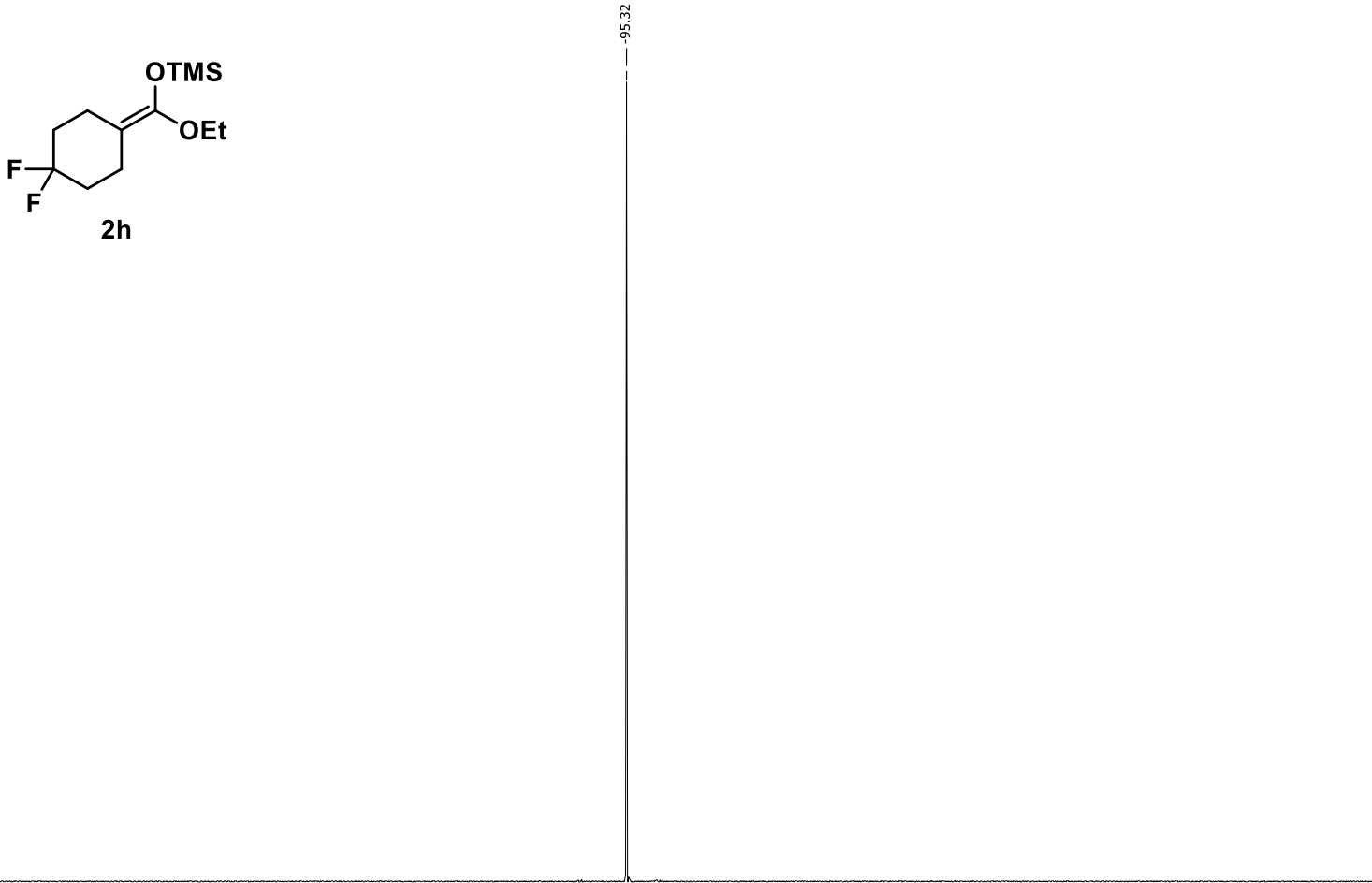

\begin{tabular}{lllllllllllllllllllllllllllllllllllllllll}
\hline 50 & 40 & 30 & 20 & 10 & 0 & -10 & -20 & -30 & -40 & -50 & -60 & -70 & -80 & -90 & -100 & -110 & -120 & -130 & -140 & -150 & -160 & -170 & -180 & -190 & -200 & -210 & -220 & -230 & -240 & $-2 !$
\end{tabular} 


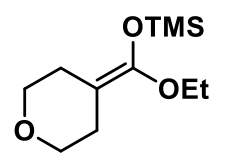

2i

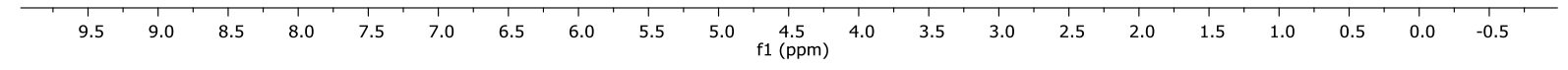

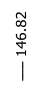

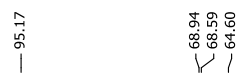

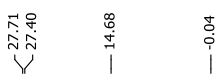

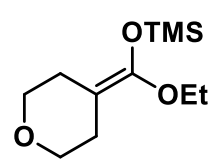

2i

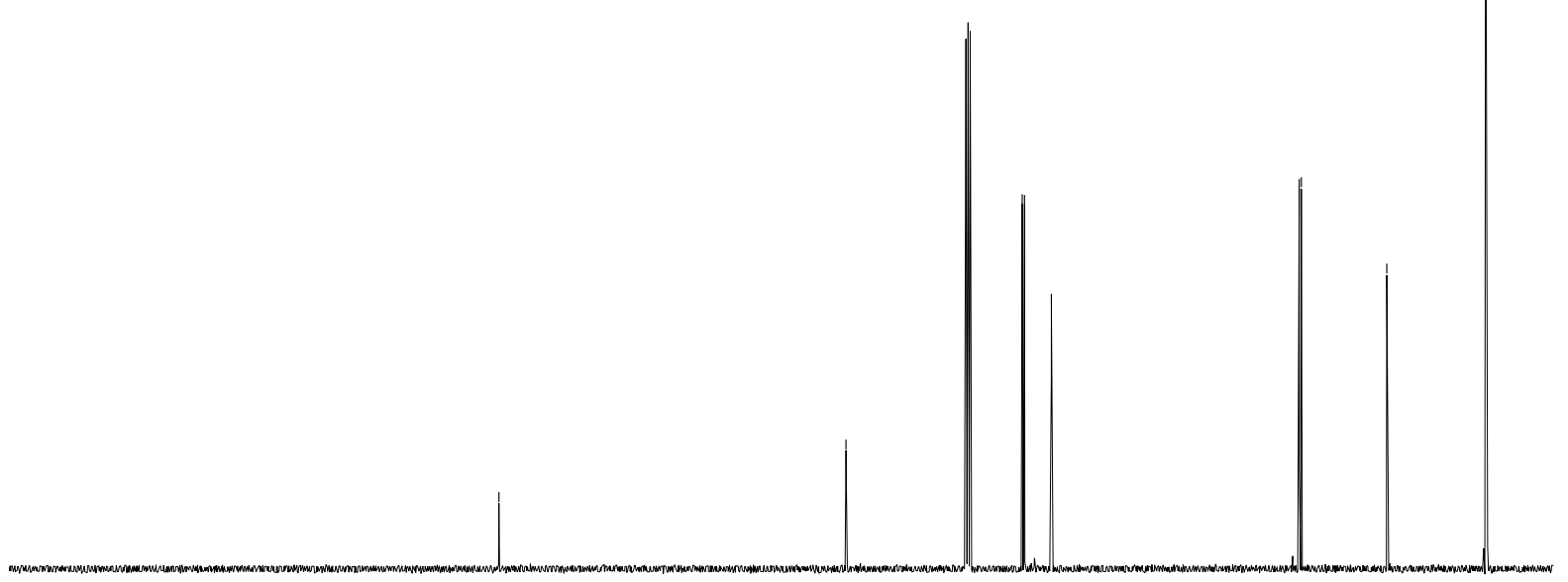

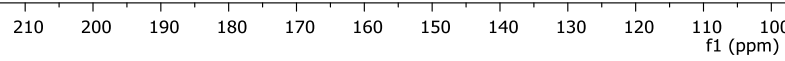



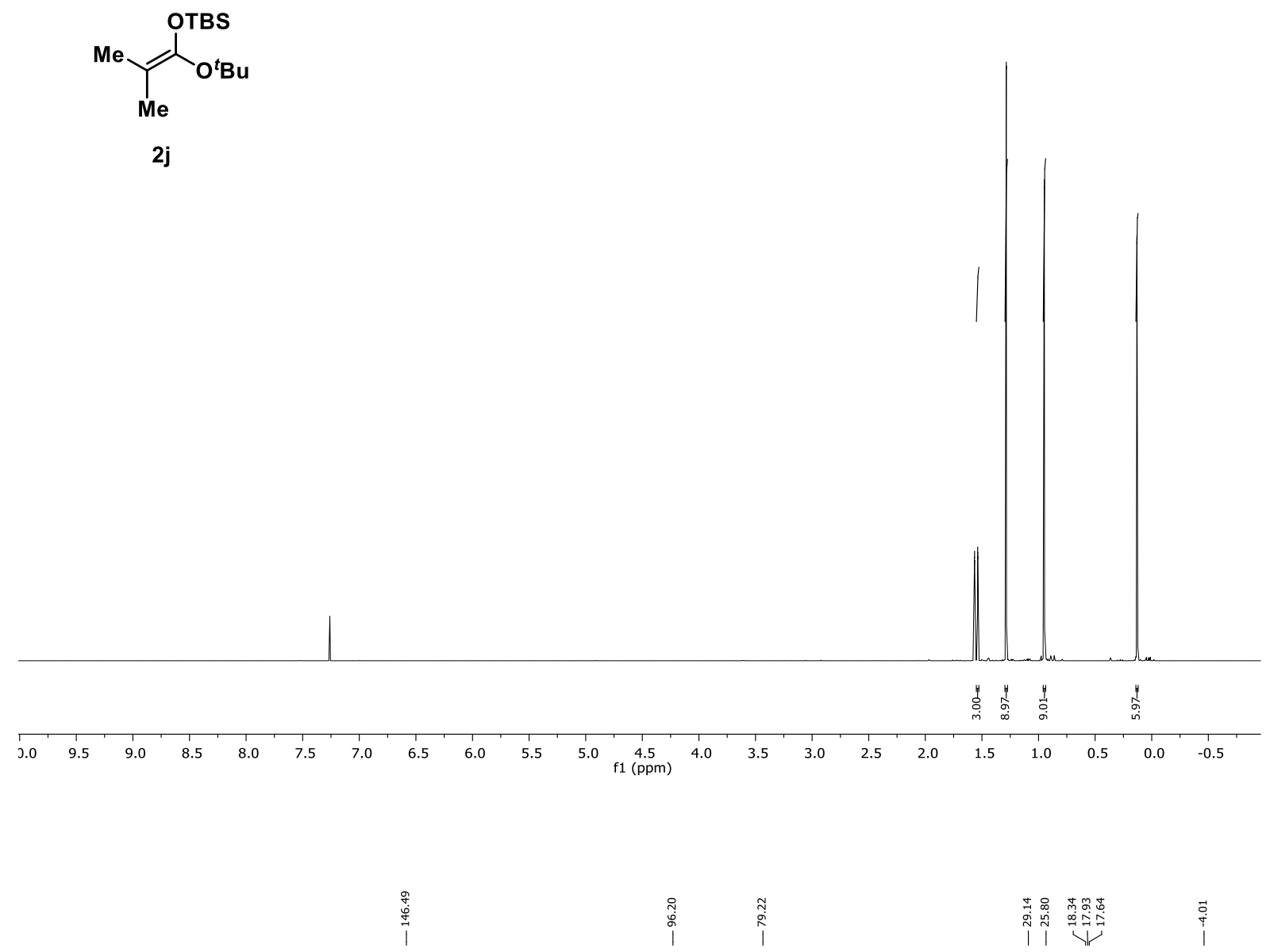<smiles>CCCCOC(O[GaH2])=C([14CH3])[N+](=O)[O-]</smiles>

2j

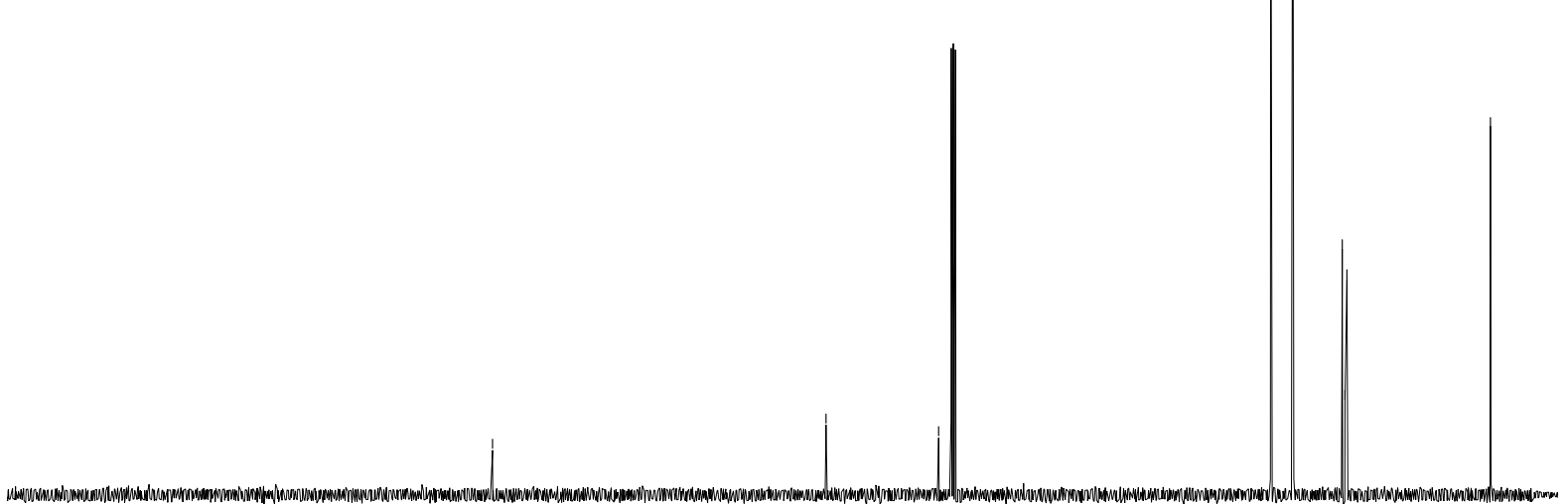

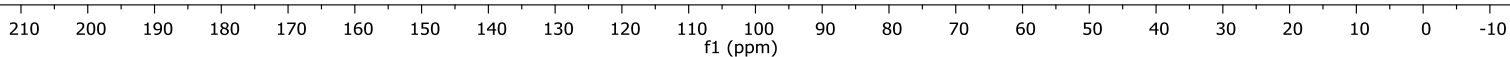




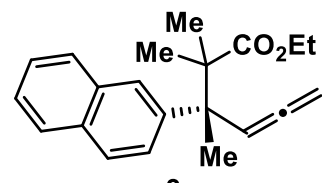

3a

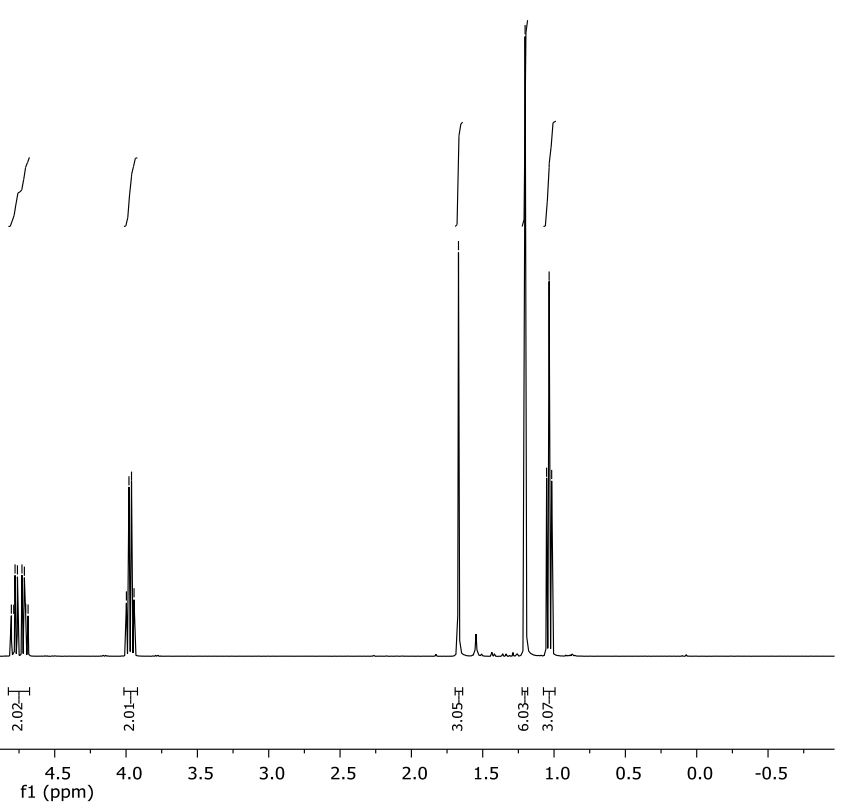

$\stackrel{\substack{0 \\ \stackrel{0}{0}}}{1}$

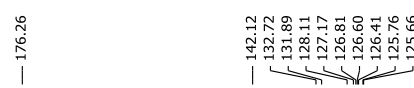

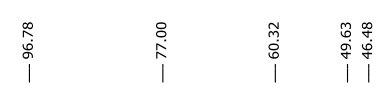

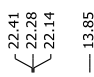
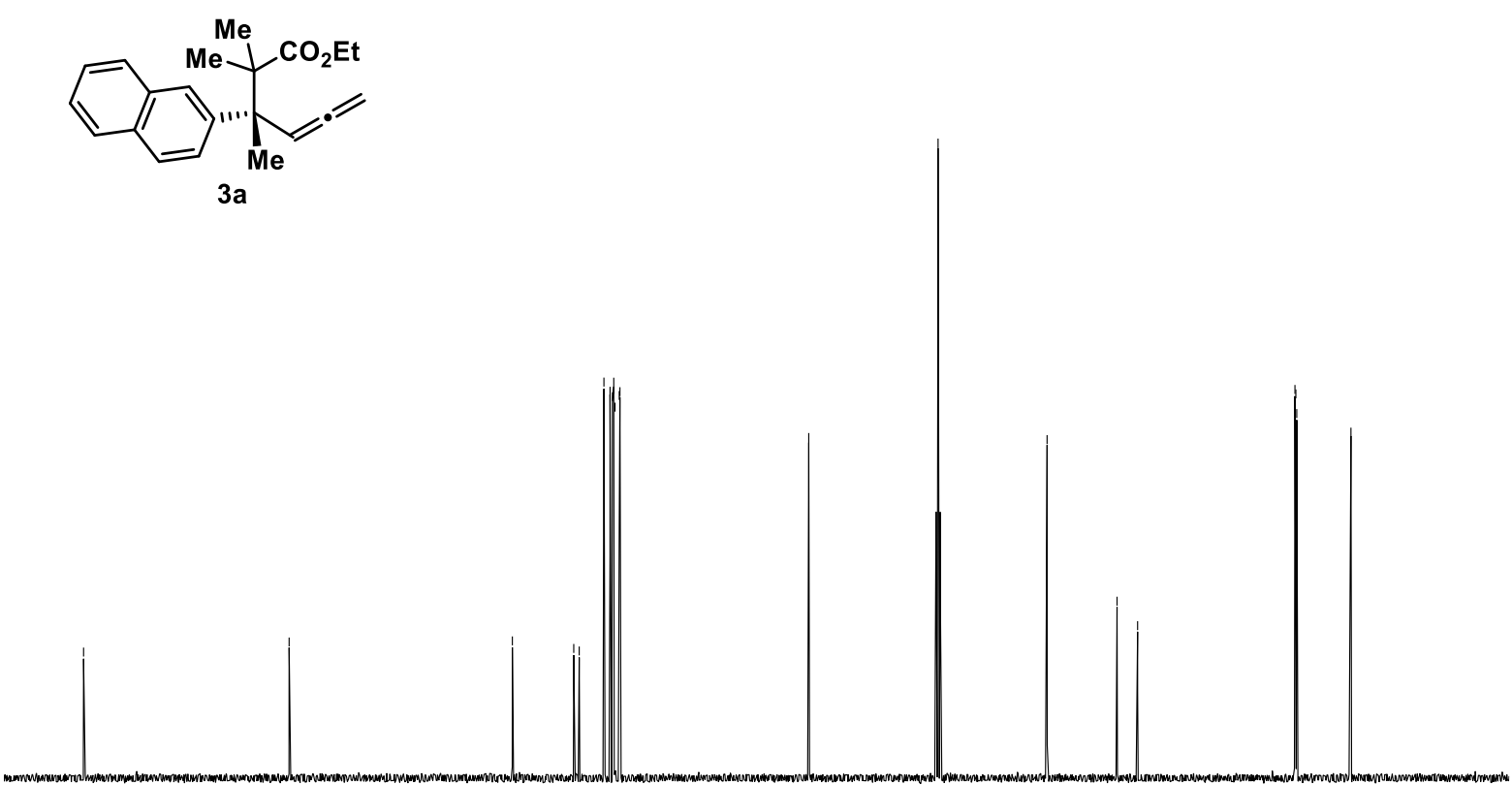

$\begin{array}{llllllllllll}210 & 200 & 190 & 180 & 170 & 160 & 150 & 140 & 130 & 120 & 110 & 100 \\ \text { f1 (ppm) }\end{array}$ 


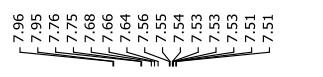

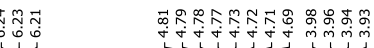

|<smiles>C=CC(C)(C)C(C)(C(C)(C)OCC)C(C)(C)c1ccc2cc(Br)ccc2c1</smiles>

3b

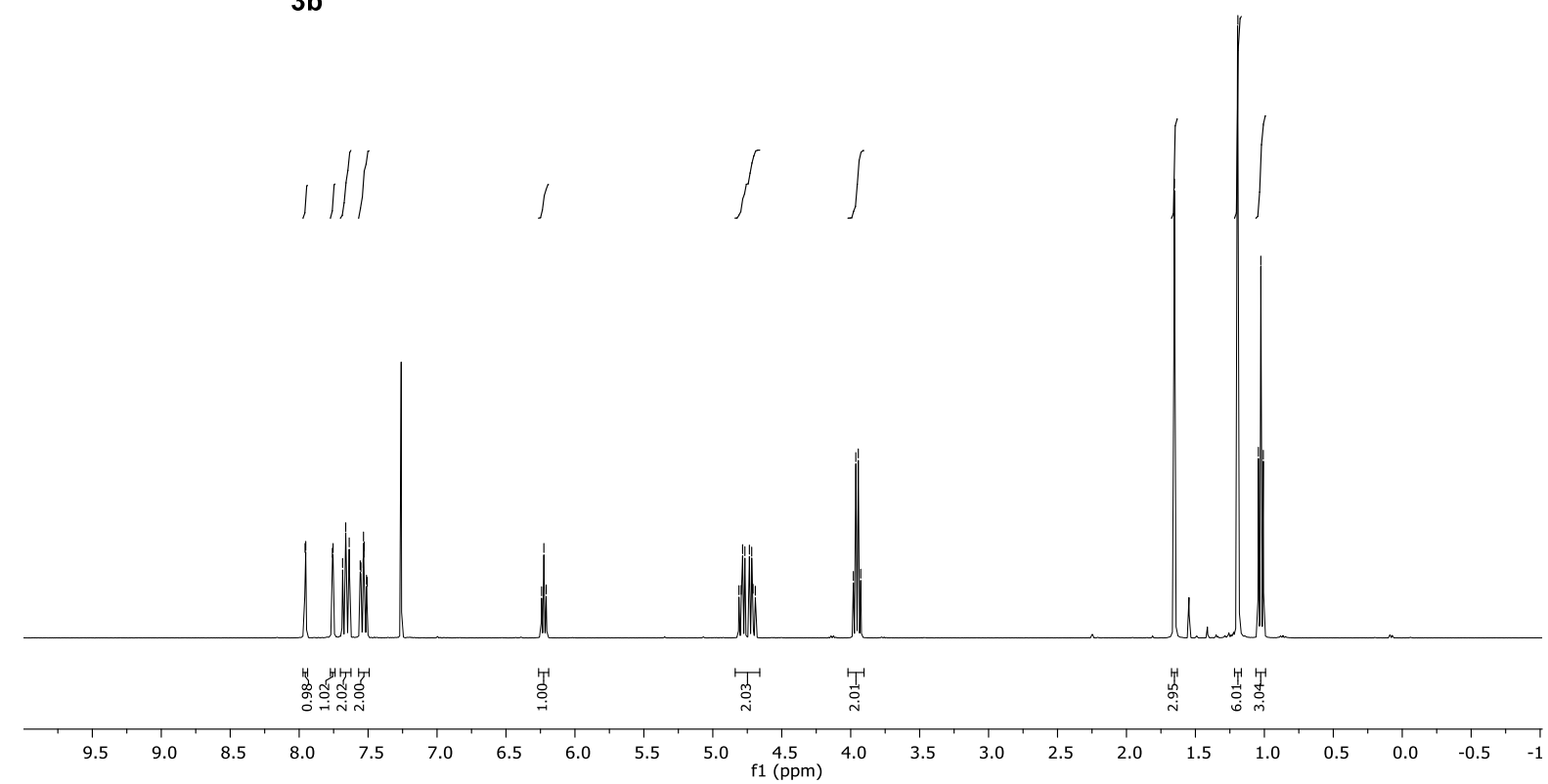

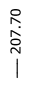

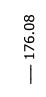

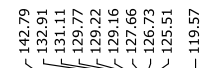

焉

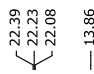

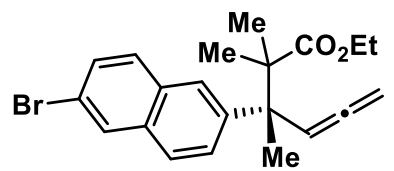

3b

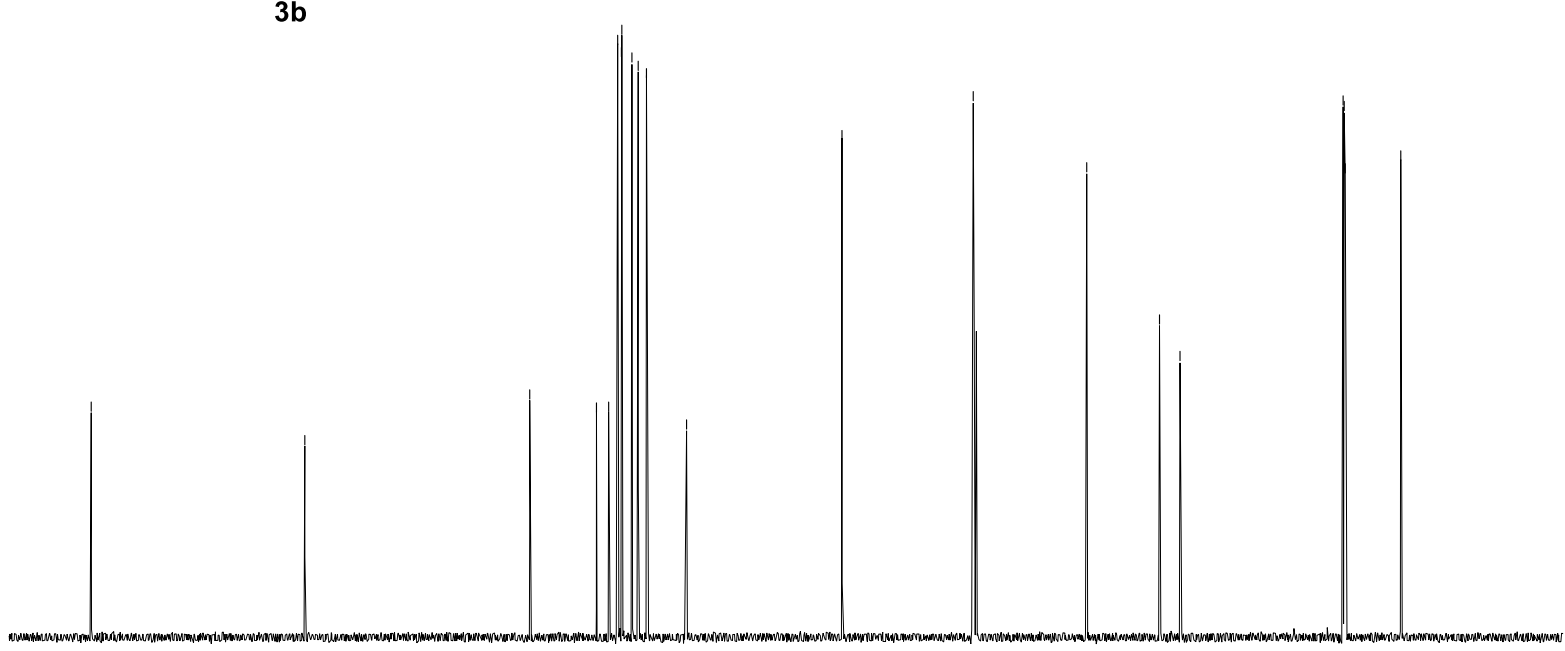

\begin{tabular}{lllllllllll}
210 & 200 & 190 & 180 & 170 & 160 & 150 & 140 & 130 & 120 & $\begin{array}{c}110 \\
\mathrm{f} 1(\mathrm{ppm})\end{array}$ \\
\hline
\end{tabular} 


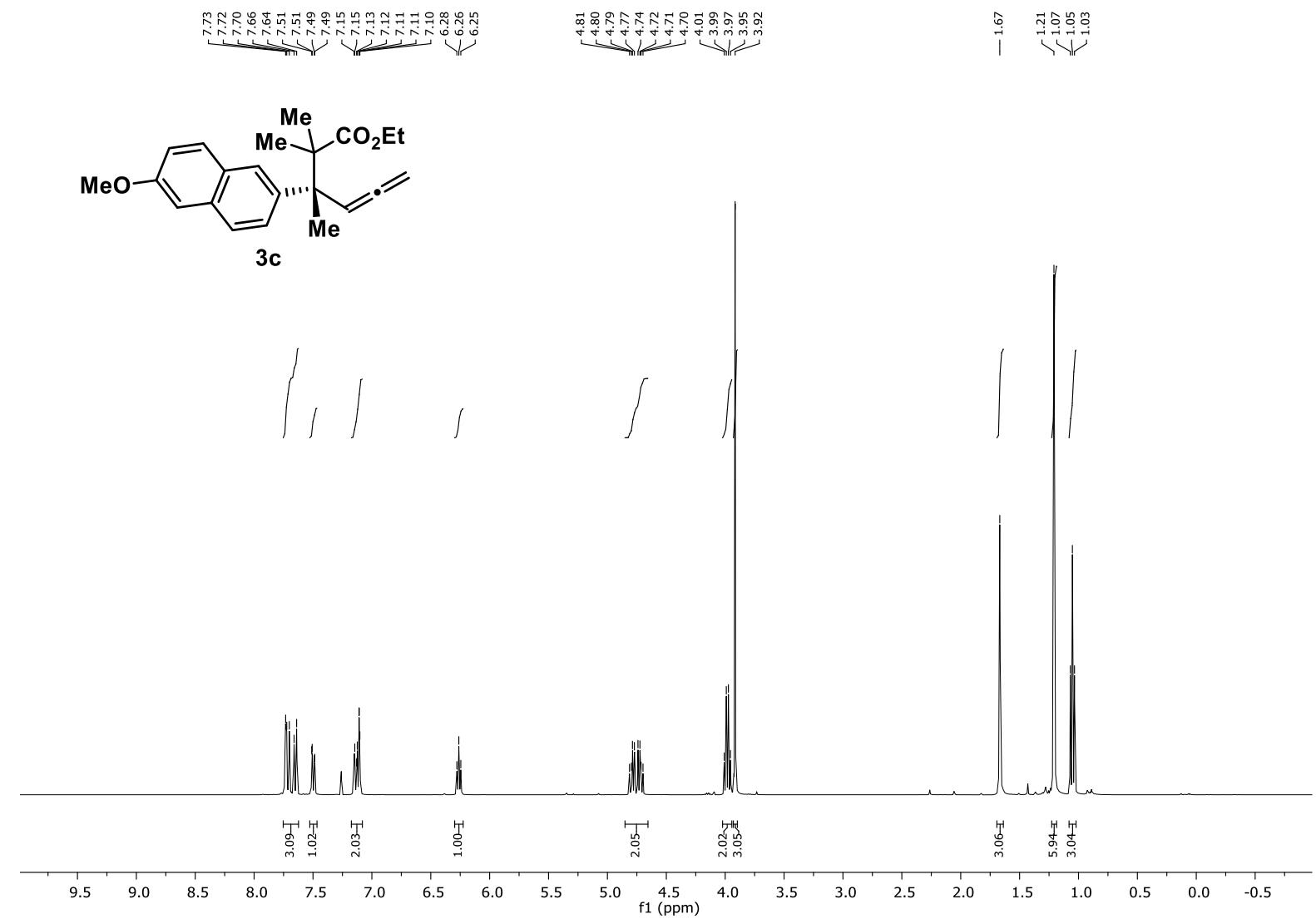

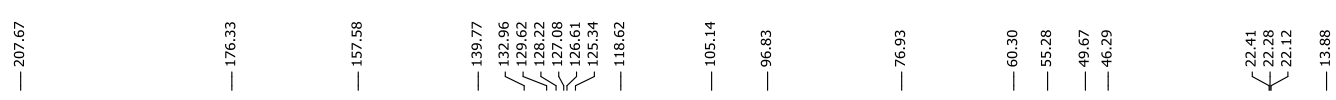
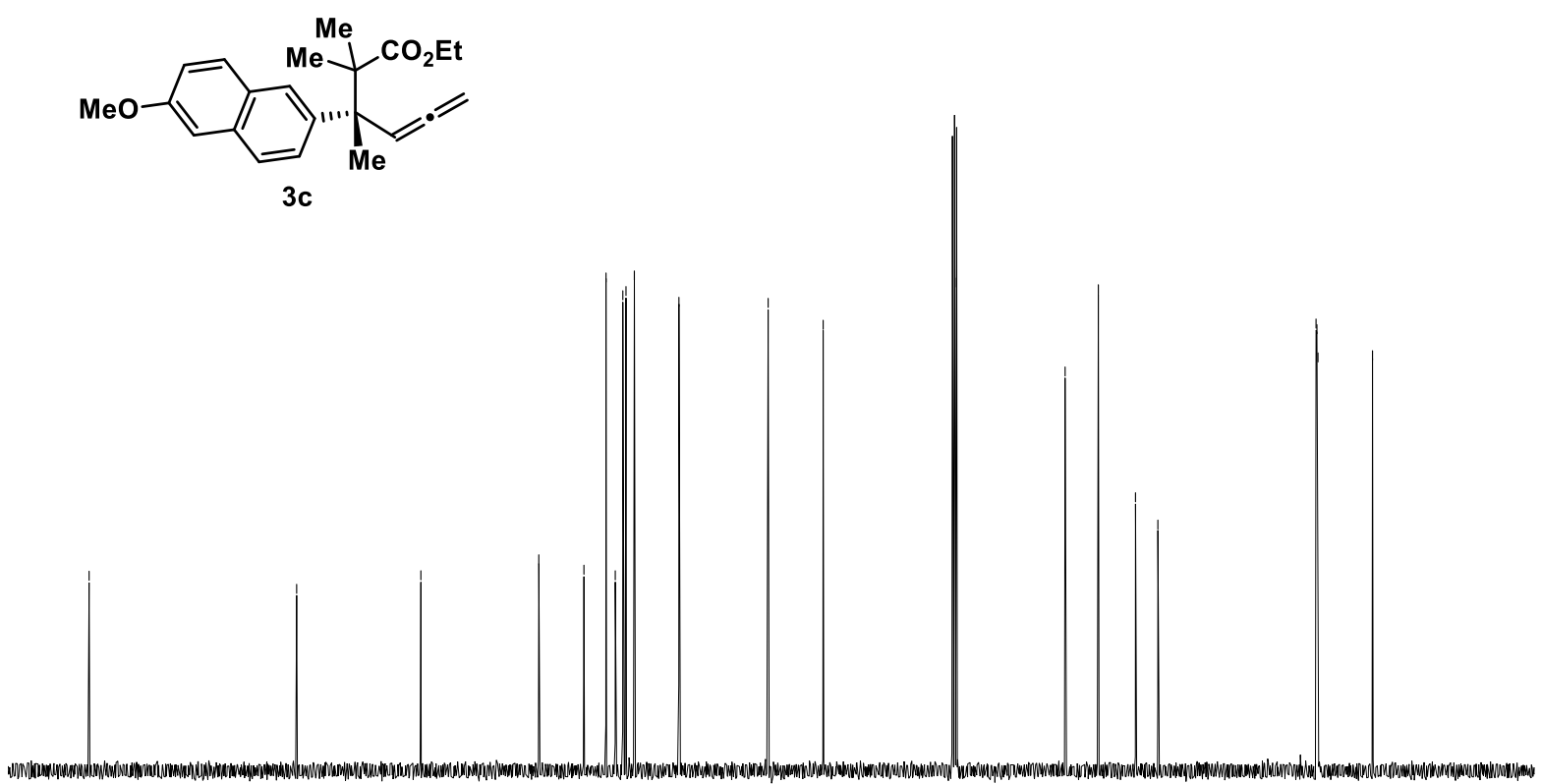

$\begin{array}{llllllllllll}210 & 200 & 190 & 180 & 170 & 160 & 150 & 140 & 130 & 120 & \begin{array}{l}110 \\ \mathrm{f} 1(\mathrm{ppm})\end{array}\end{array}$ 


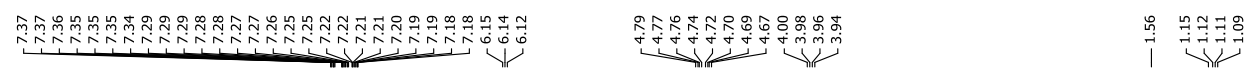

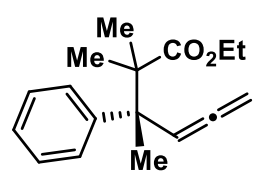

$3 d$

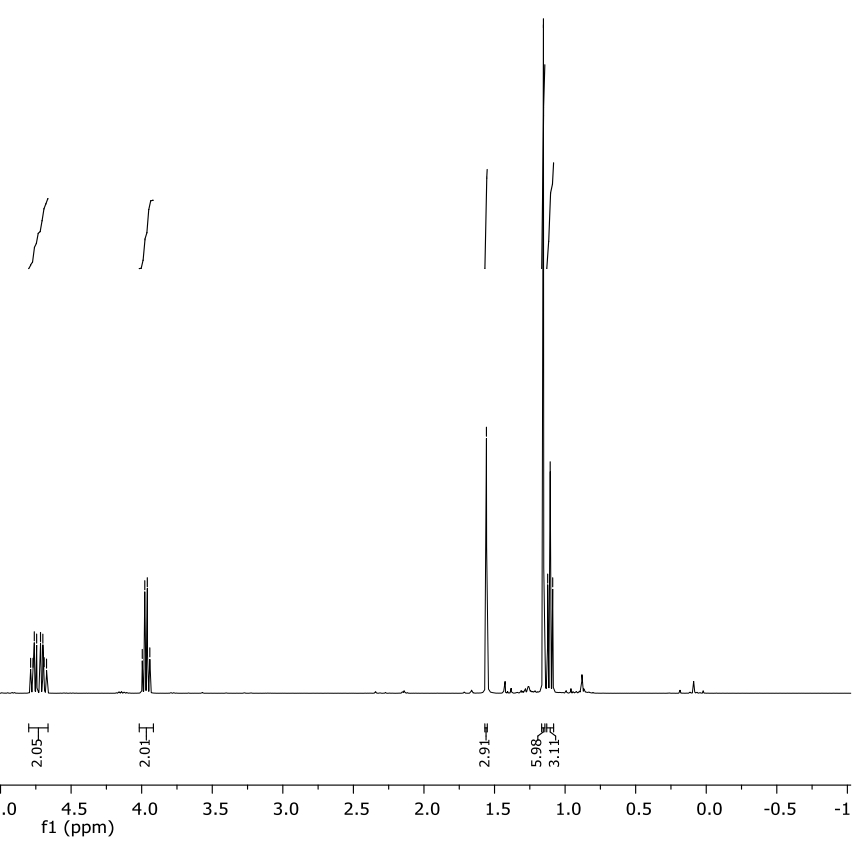

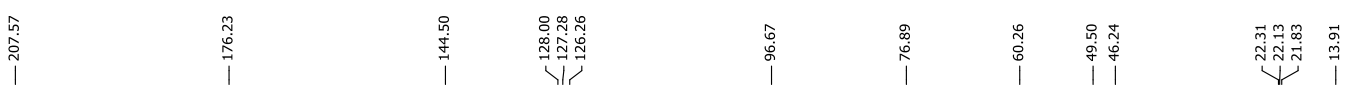

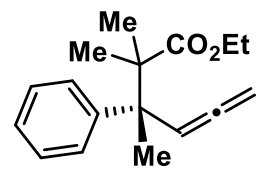

$3 d$

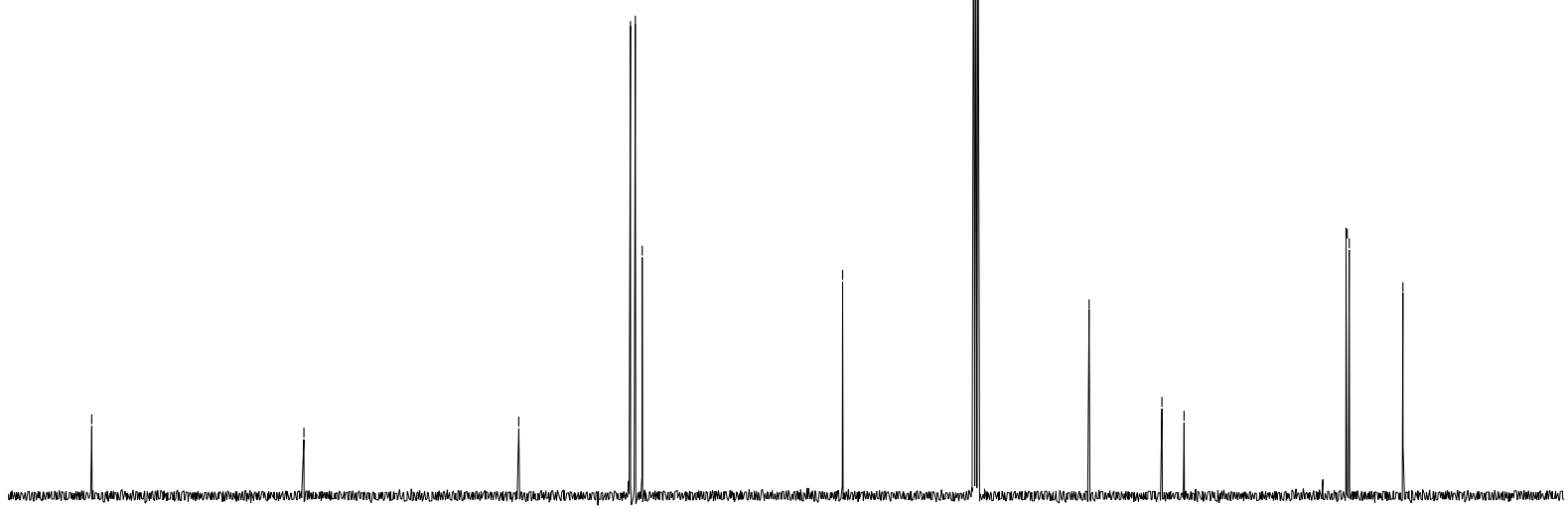

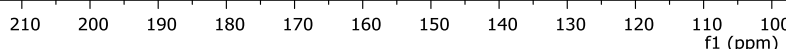




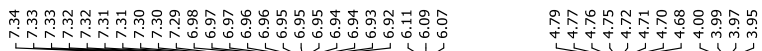

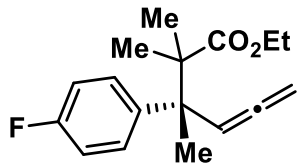

$3 e$

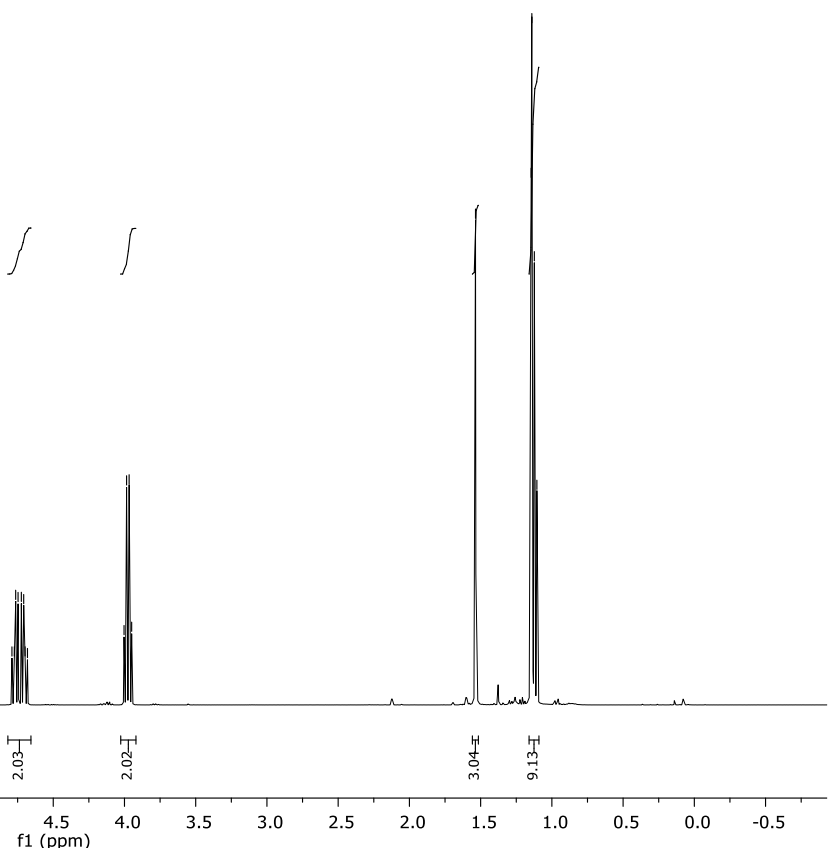

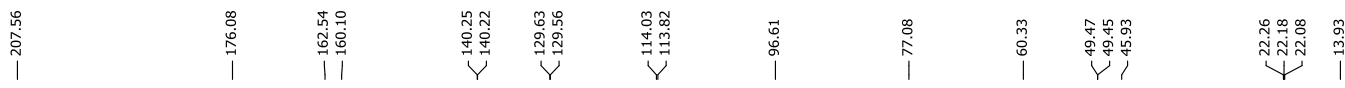

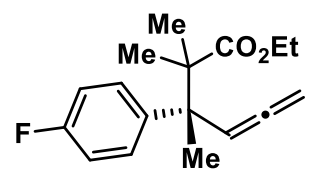

$3 e$

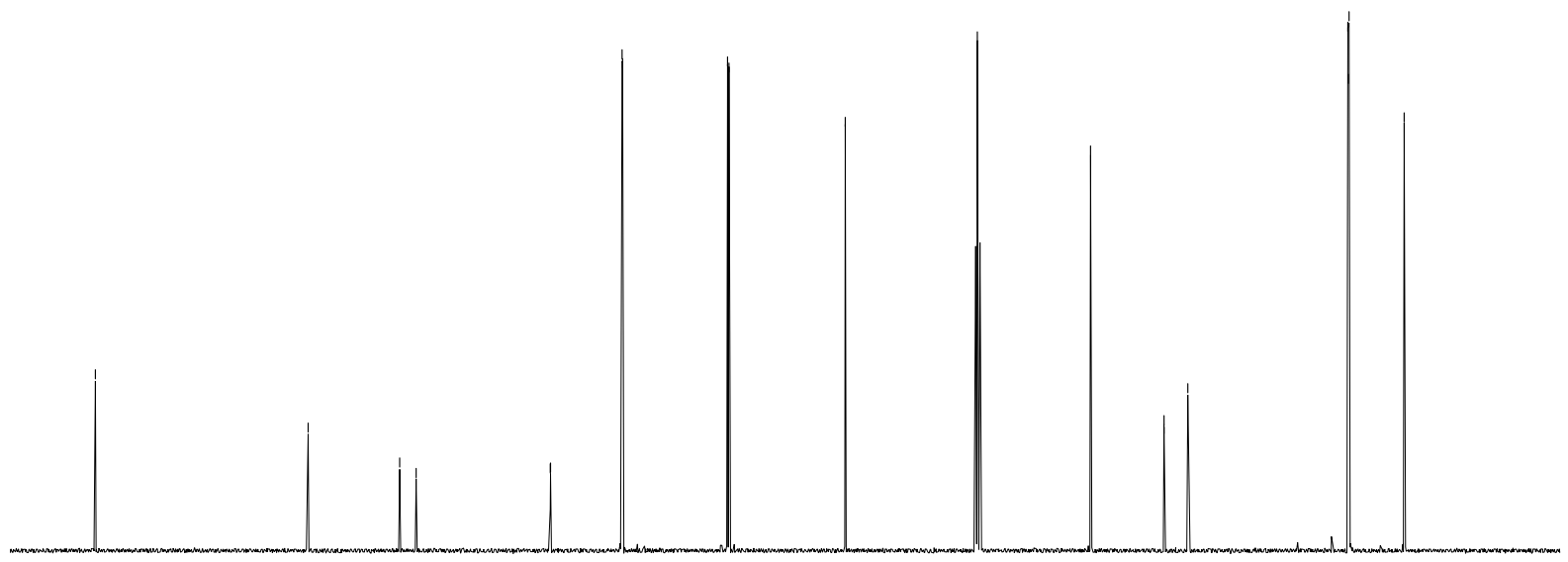

$\begin{array}{llllllllllllllllllllllllllllll}1 & 210 & 200 & 190 & 180 & 170 & 160 & 150 & 140 & 130 & 120 & 110 & 100 & 90 & 80 & 70 & 60 & 50 & 40 & 30 & 20 & 10 & 0 & 1\end{array}$ 


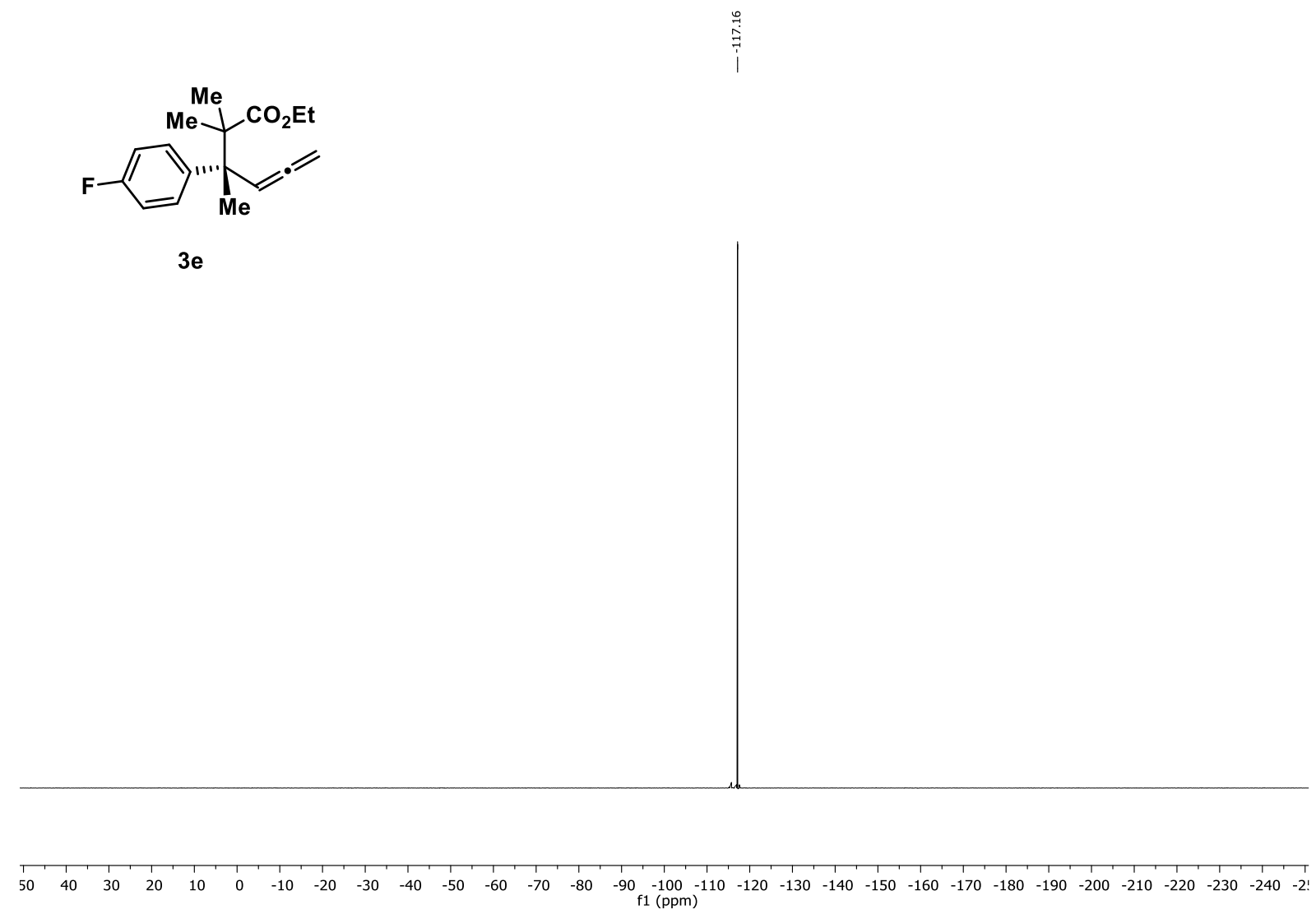




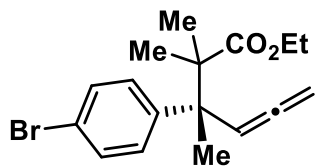

$3 f$

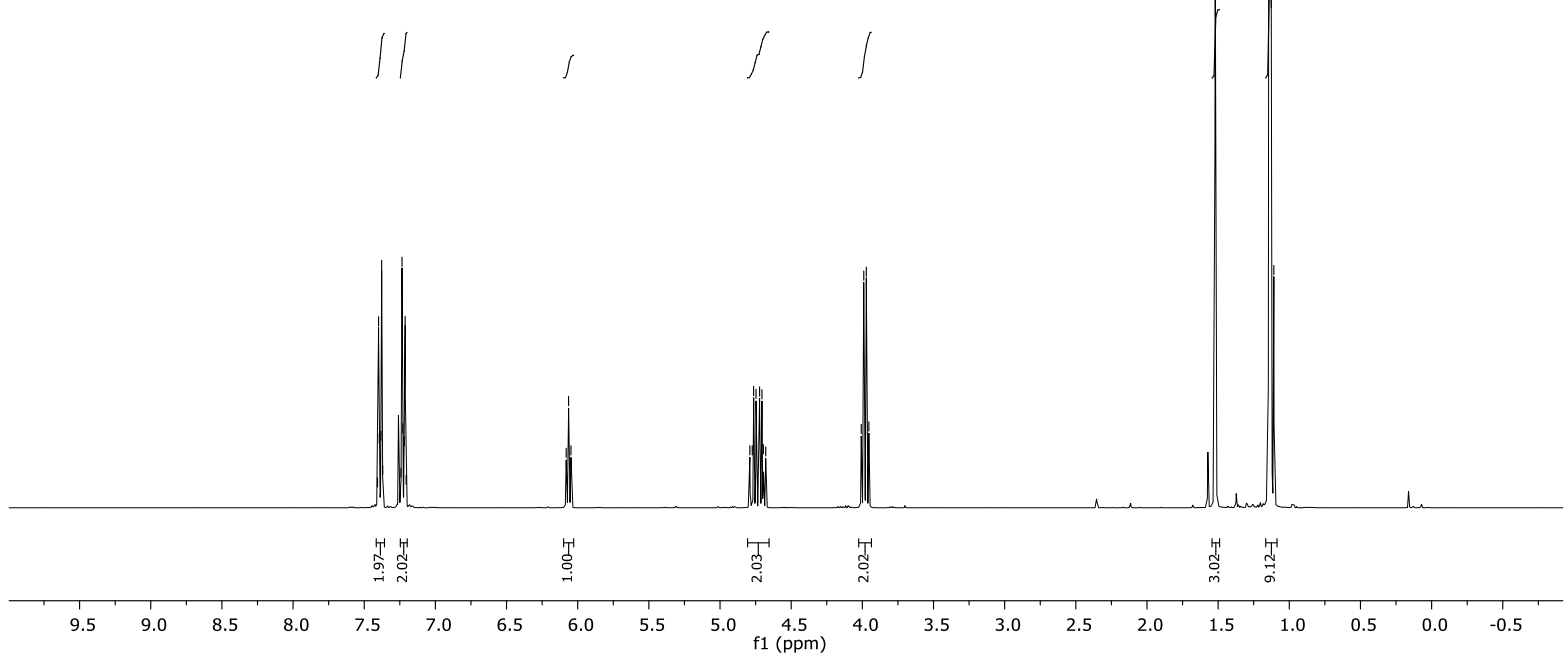

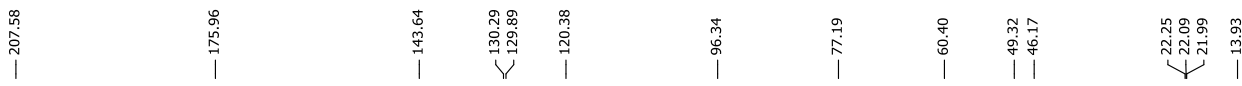

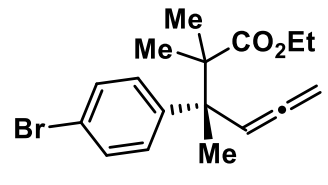

$3 f$

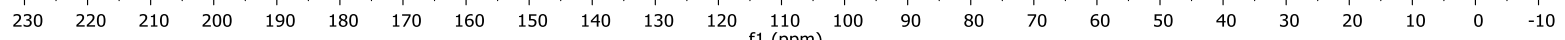




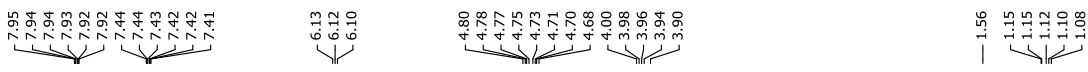

$\mathrm{MeO}_{2} \mathrm{C}$

$3 g$<smiles>CCC1CCC1</smiles>

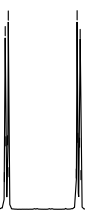

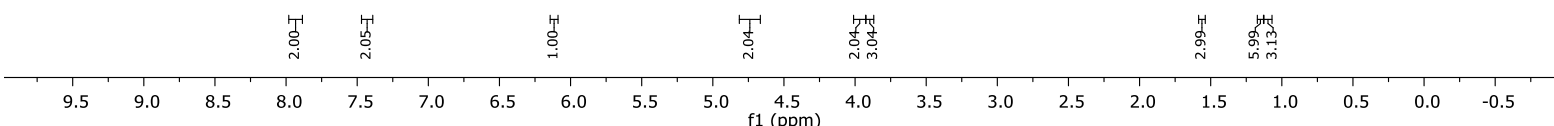

$3 \mathrm{~g}$

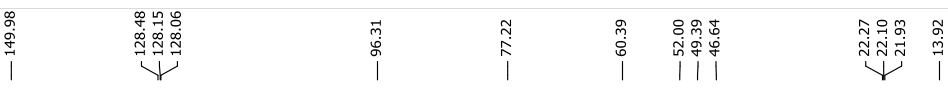
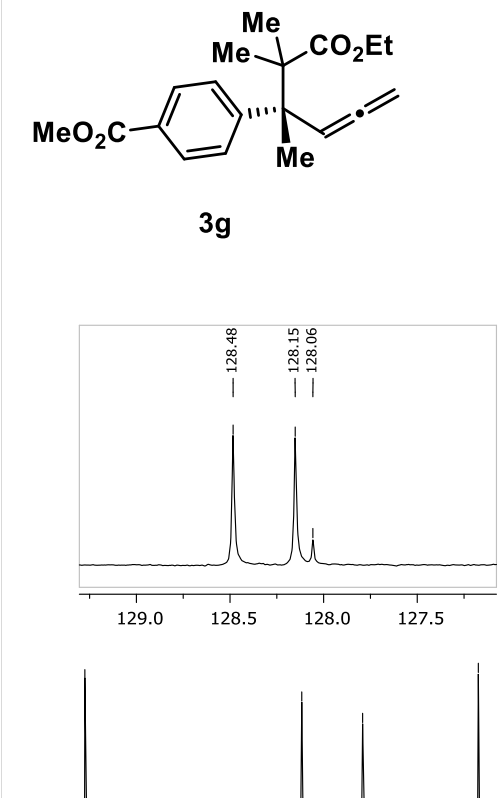

$\begin{array}{llllllllllll}210 & 200 & 190 & 180 & 170 & 160 & 150 & 140 & 130 & 120 & 110 & 100\end{array}$ 


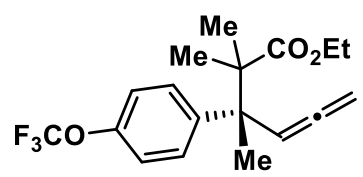

$3 h$

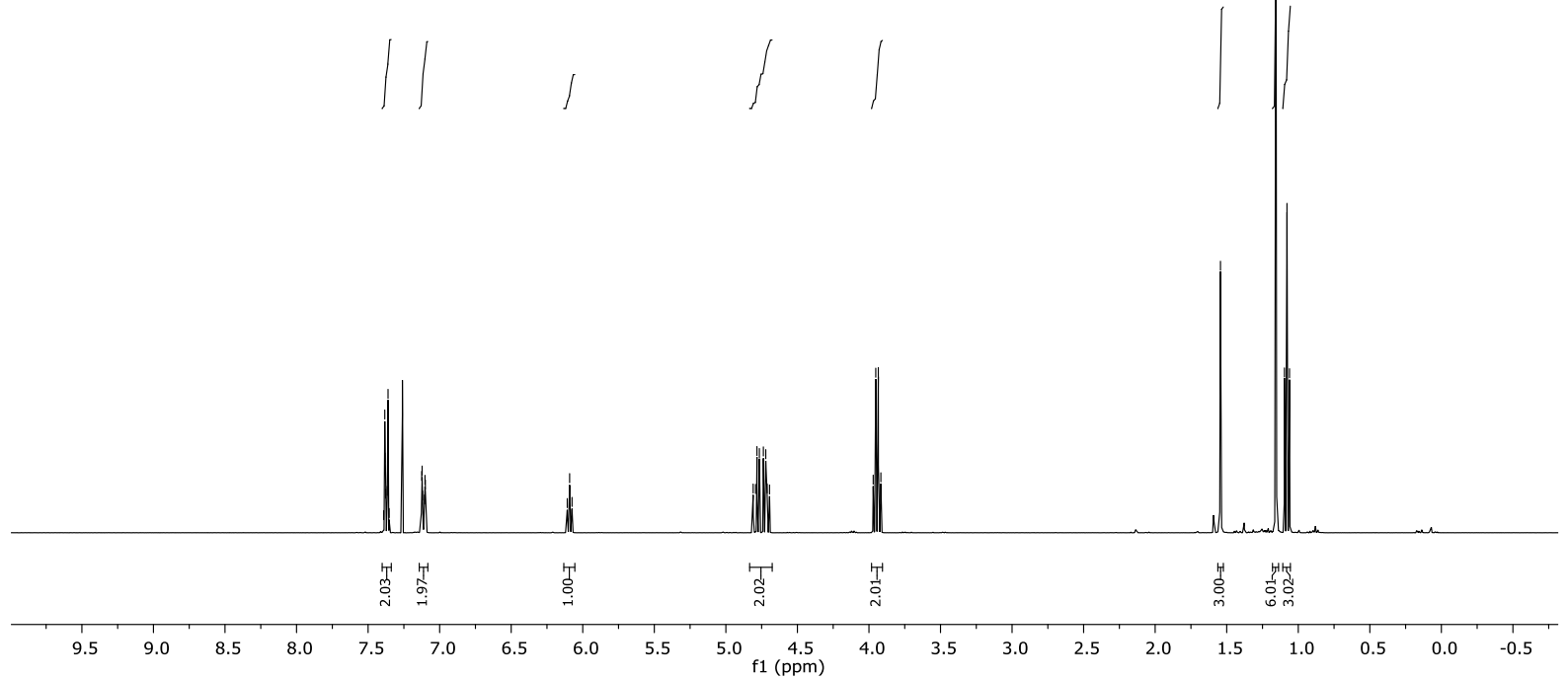

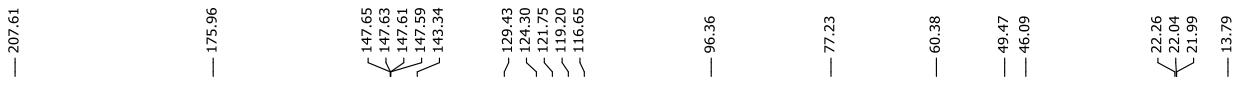

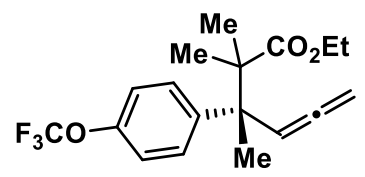

3h

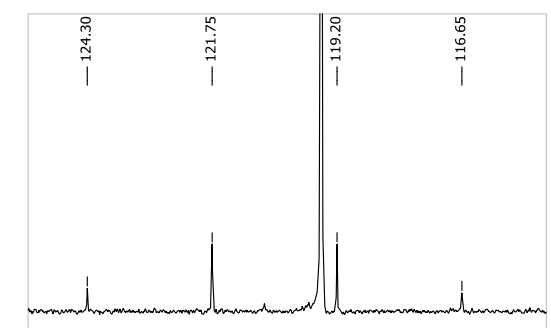

\begin{tabular}{lllllllllllll}
\hline 125 & 124 & 123 & 122 & 121 & 120 & 119 & 118 & 117 & 116 & 115 \\
\hline
\end{tabular}

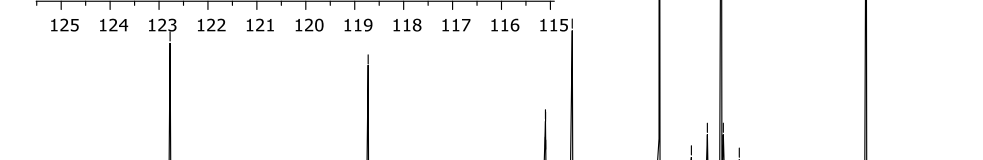

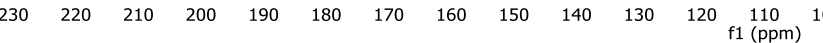




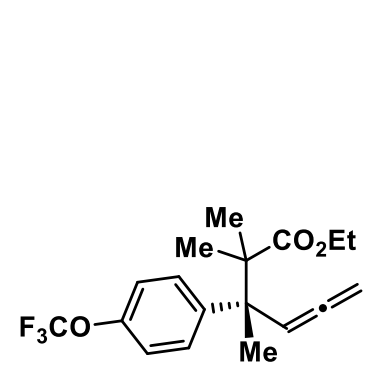

$3 h$

$\begin{array}{lllllllllllllllllllllllllllllllllllllllllllll}50 & 40 & 30 & 20 & 10 & 0 & -10 & -20 & -30 & -40 & -50 & -60 & -70 & -80 & -90 & -100 & -110 & -120 & -130 & -140 & -150 & -160 & -170 & -180 & -190 & -200 & -210 & -220 & -230 & -240 & -2 !\end{array}$ 


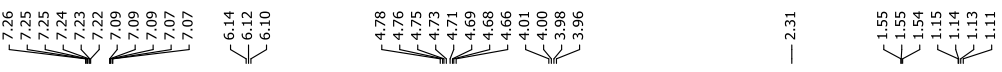

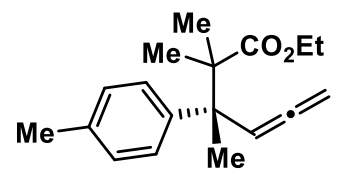

$3 \mathbf{i}$

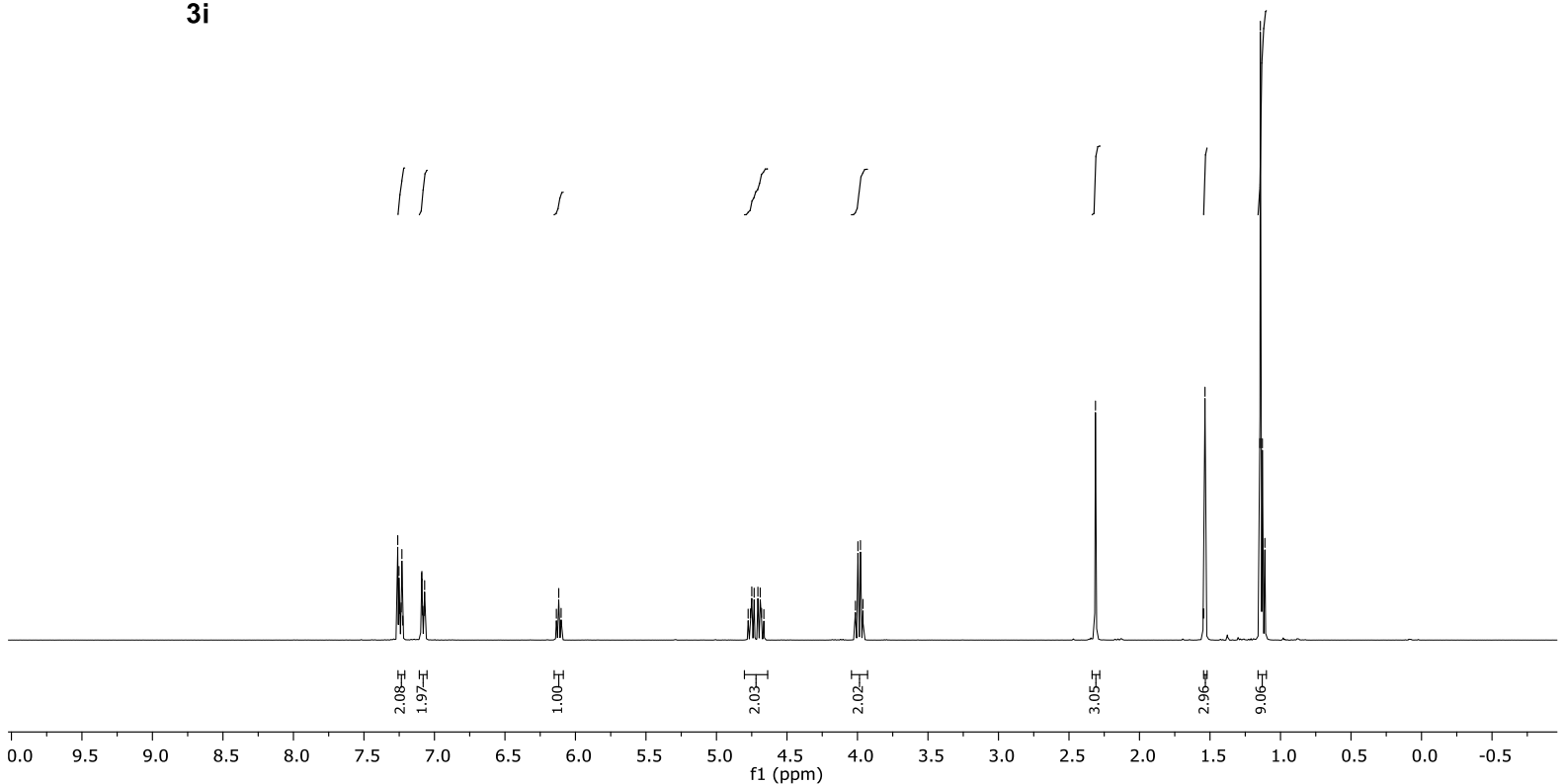

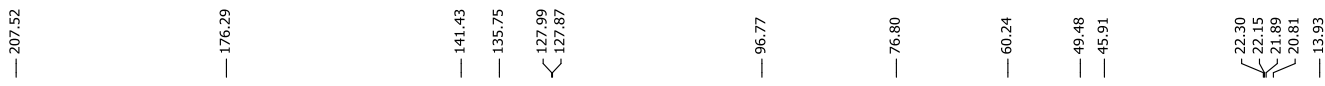

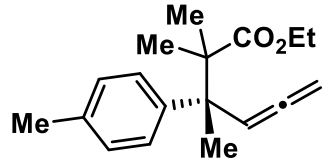

$3 \mathbf{i}$ 


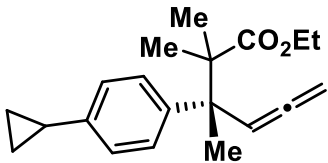

3j

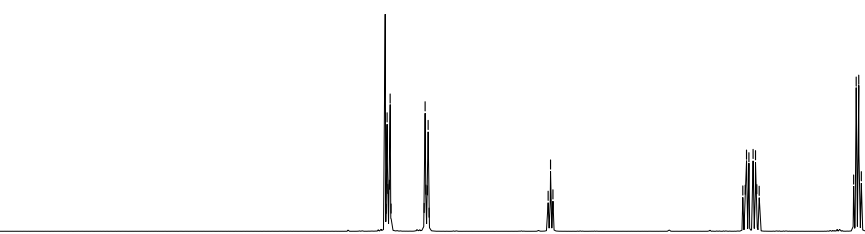

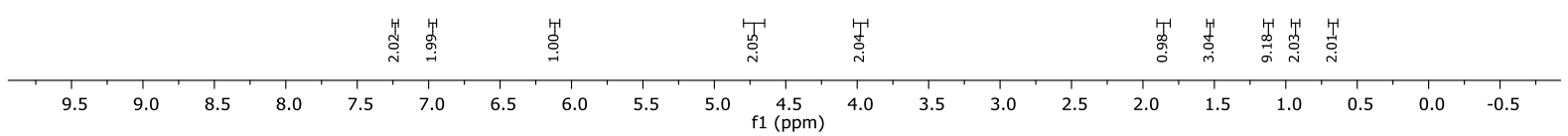

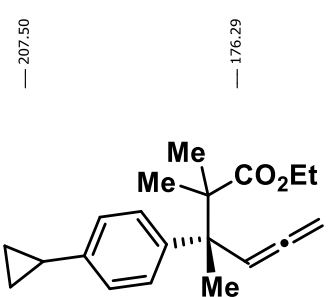

3j

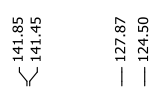

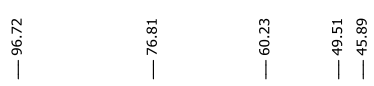

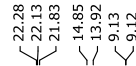

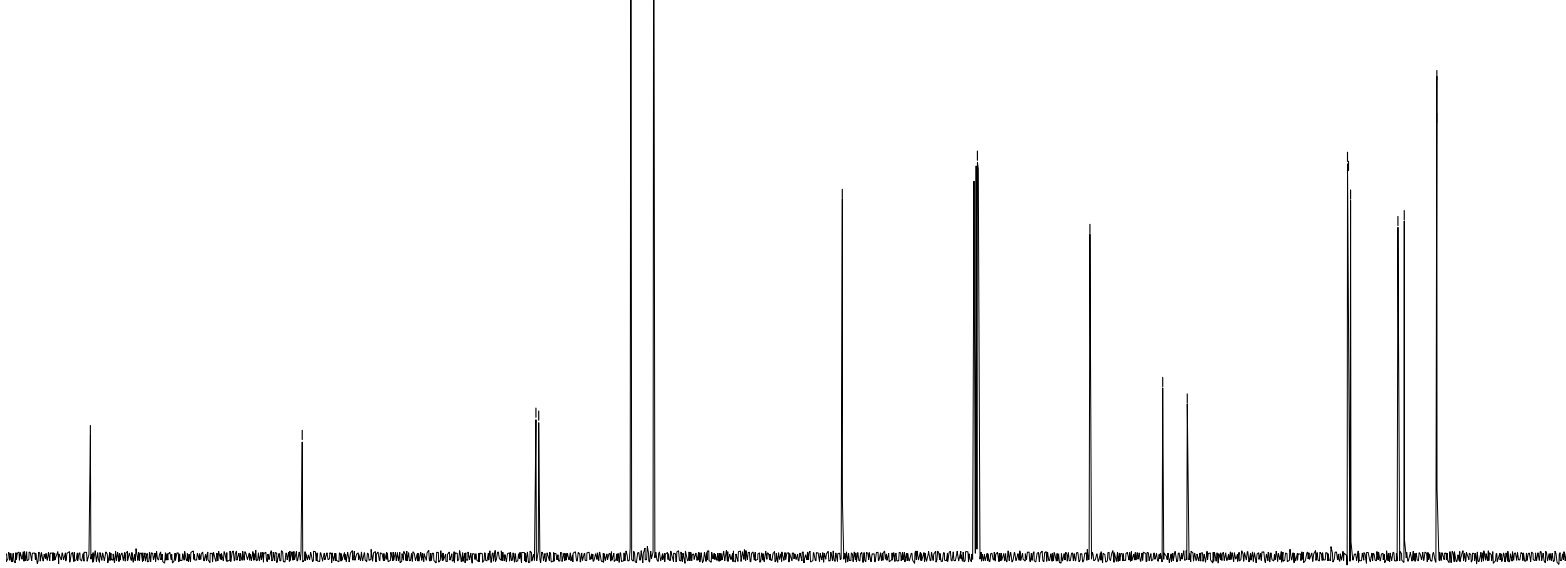

$\begin{array}{lllllllllll}210 & 200 & 190 & 180 & 170 & 160 & 150 & 140 & 130 & 120 & \begin{array}{l}110 \\ \mathrm{f} 1(\mathrm{ppm})\end{array}\end{array}$ 


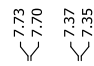

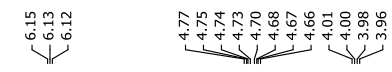

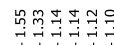

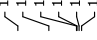

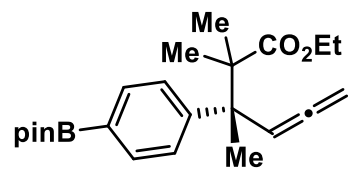

3k

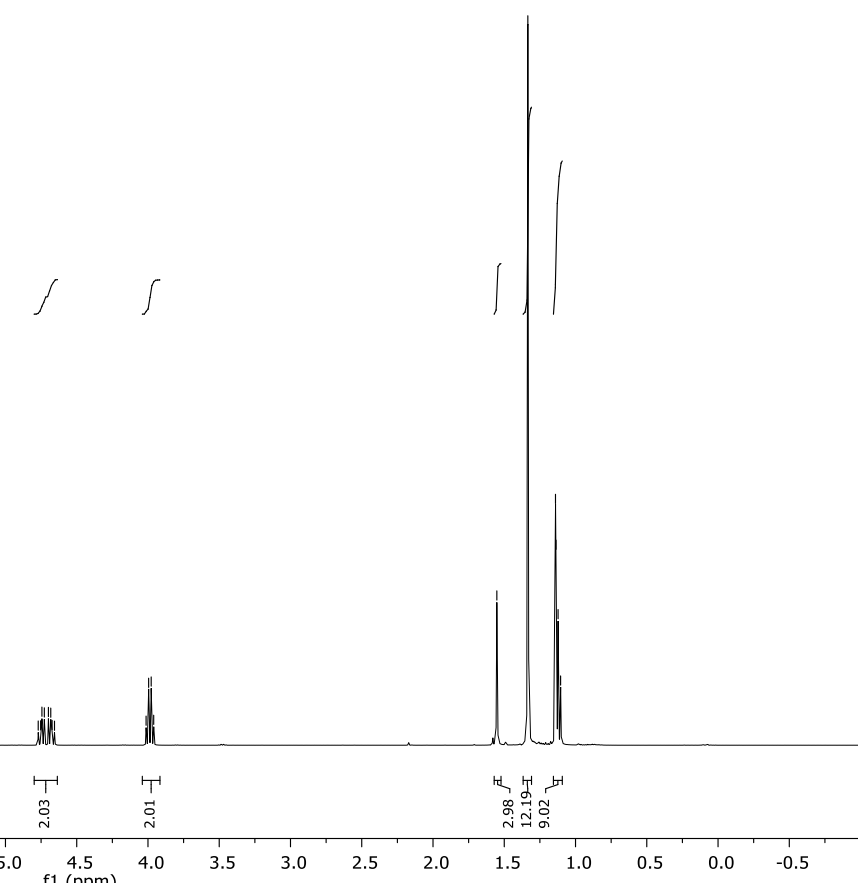

$\stackrel{\overrightarrow{0}}{\stackrel{N}{\hat{~}}}$

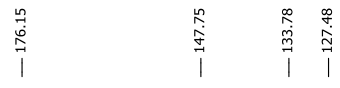

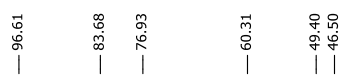

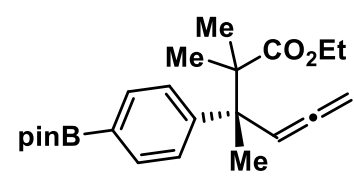

$3 k$

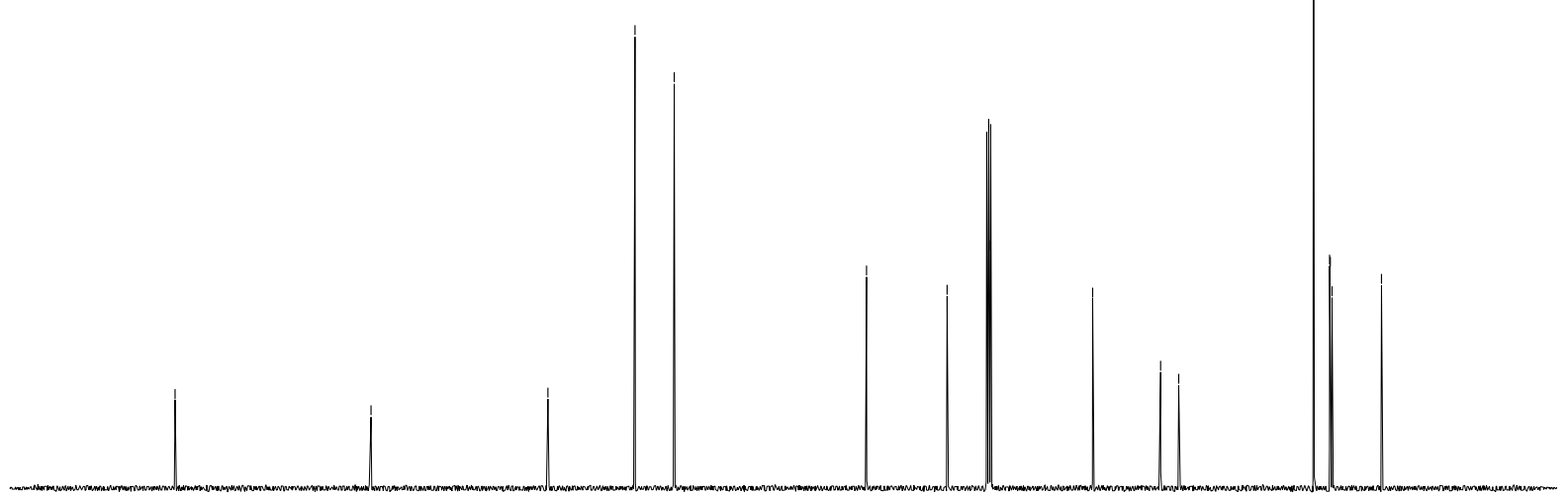

$\begin{array}{lllllllllllllllllllllllllllllllllllllll}230 & 220 & 210 & 200 & 190 & 180 & 170 & 160 & 150 & 140 & 130 & 120 & 110 & 100 & 90 & 80 & 70 & 60 & 50 & 40 & 30 & 20 & 10 & 0 & -10\end{array}$ 


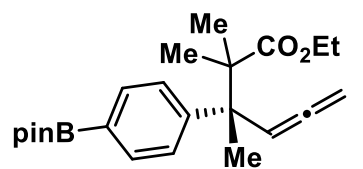

$3 k$

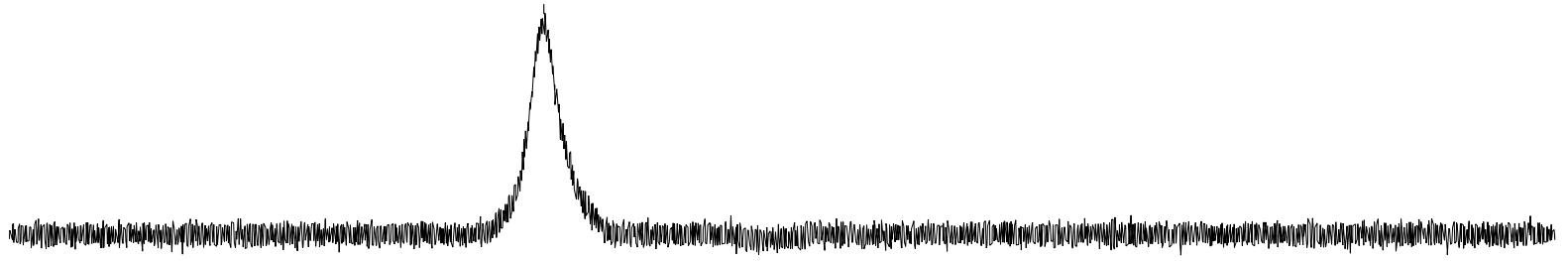

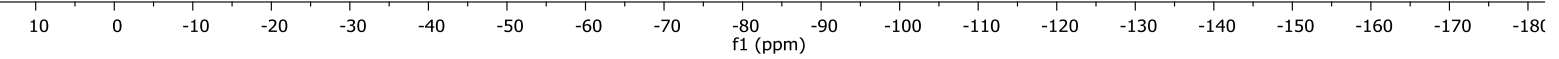




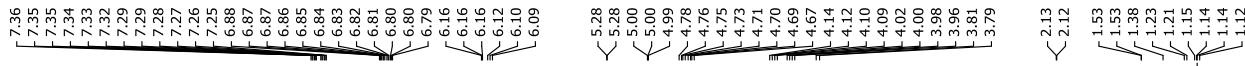

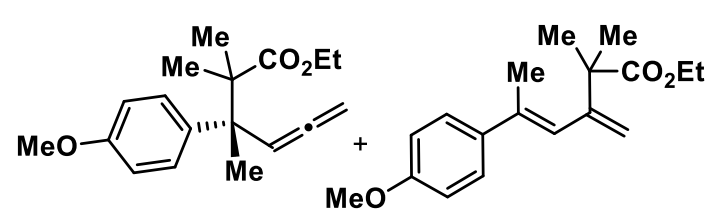

3I

3l'

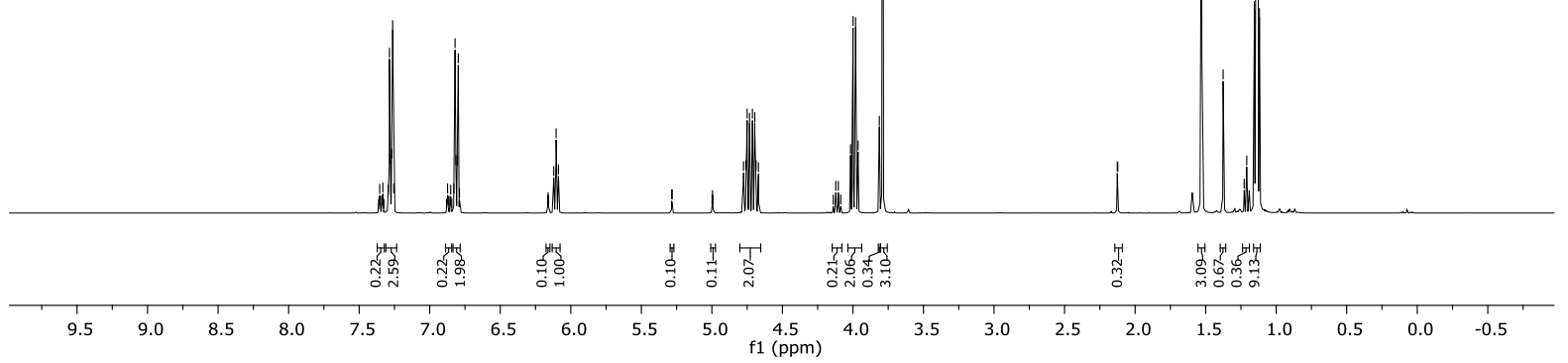

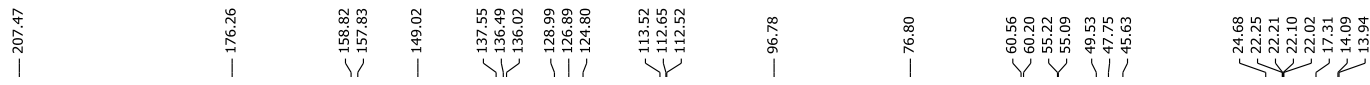

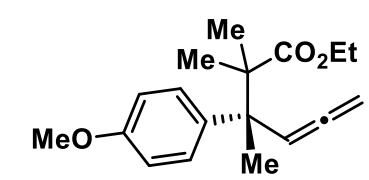

3)

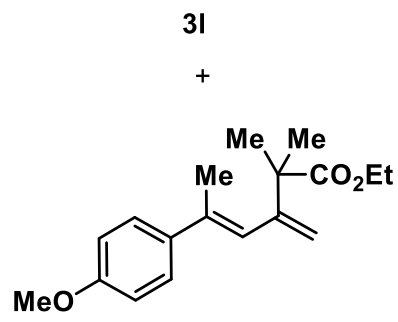

$31^{\prime}$

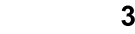

$\int$

$\int$

$\int$
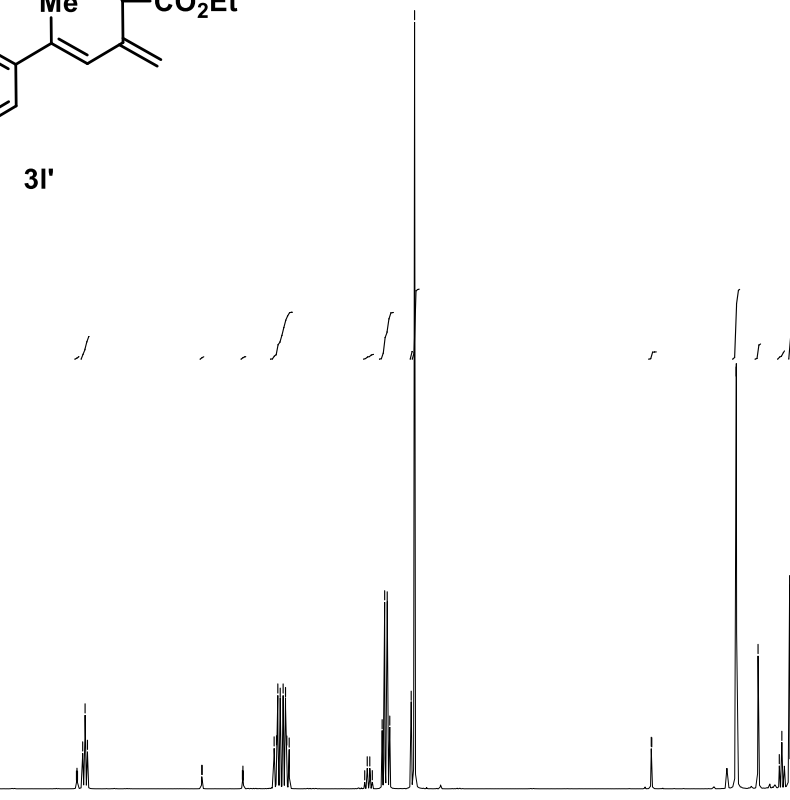


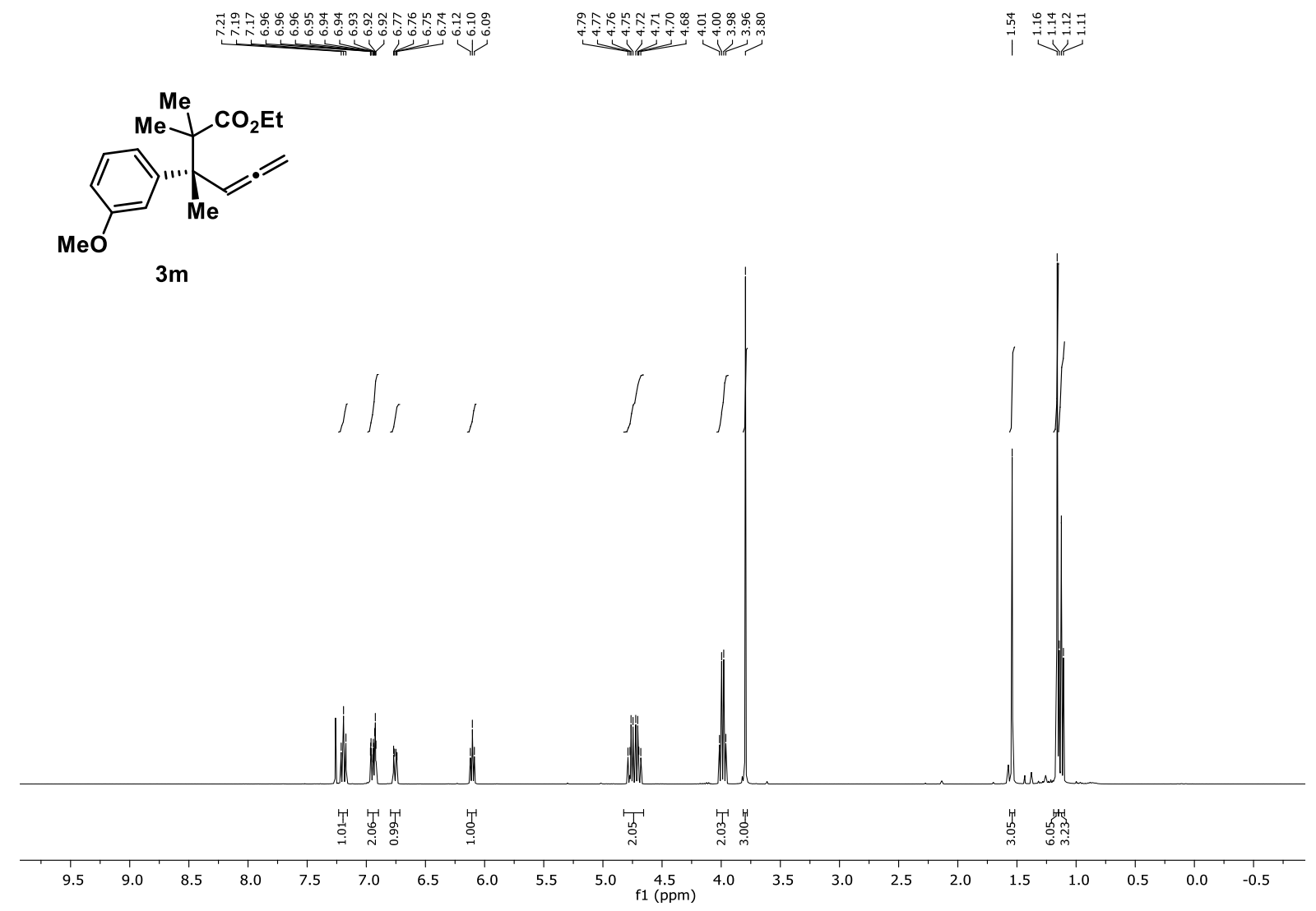

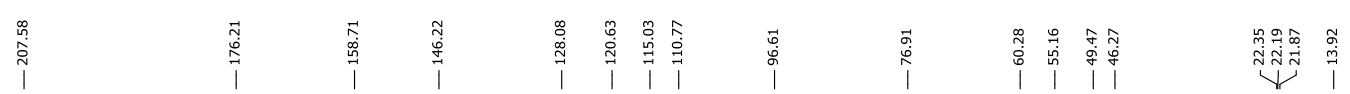

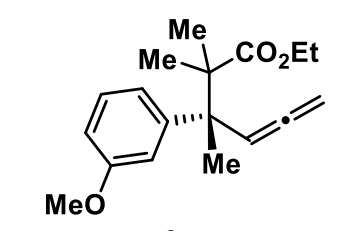

$3 m$

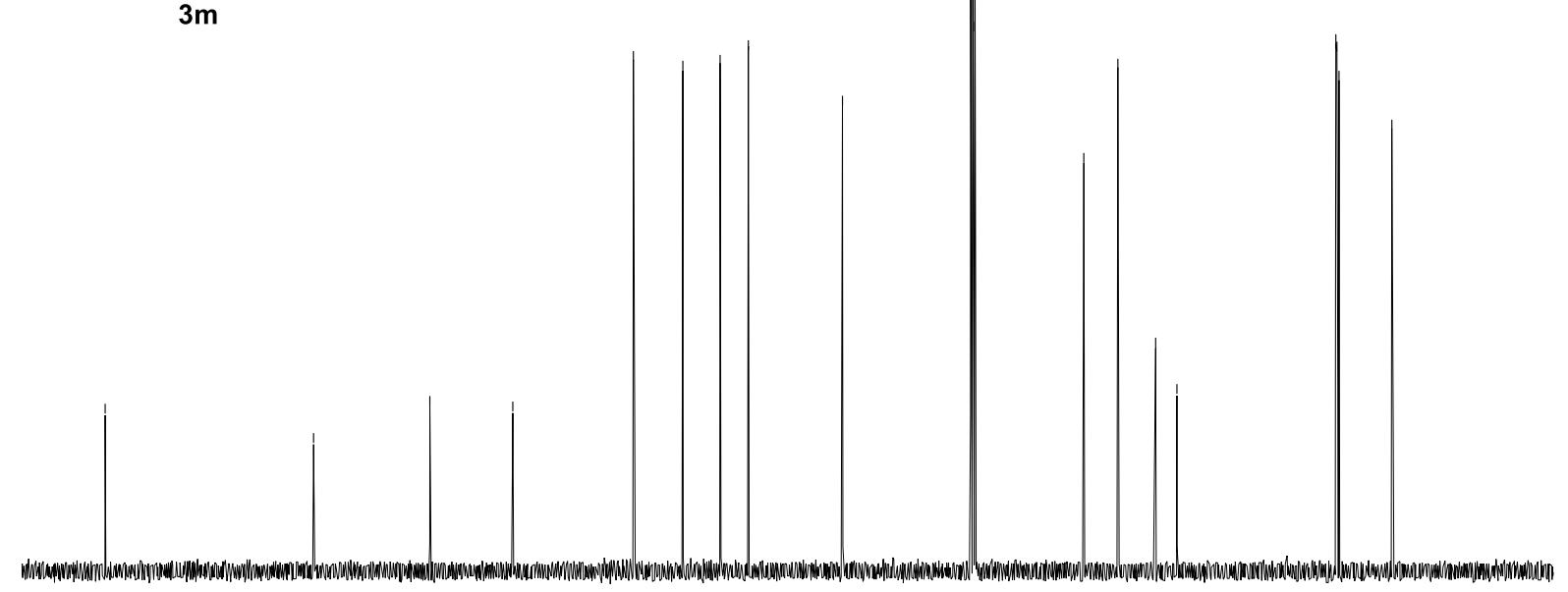

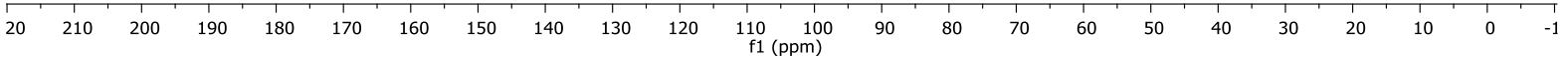


<smiles>C=C=CC(c1ccccc1OC)(c1ccccc1OC)C(C)(C)C(=O)OCC</smiles>

$3 n$

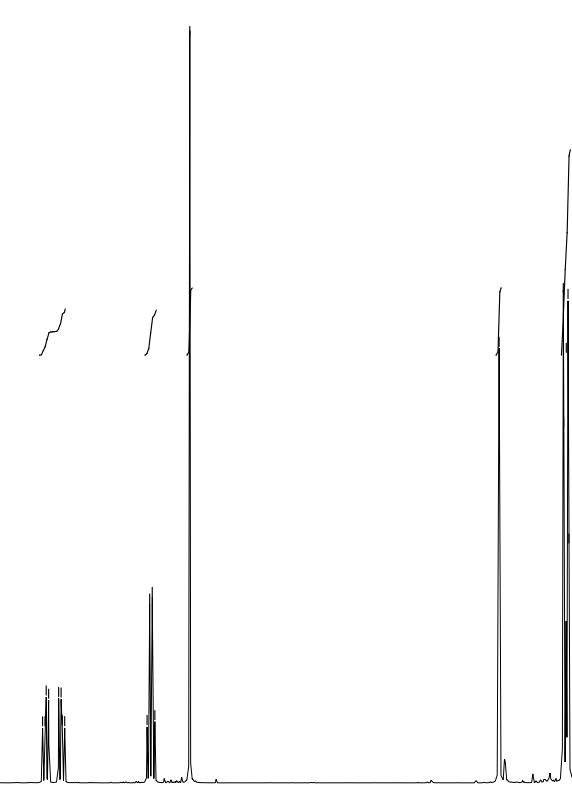

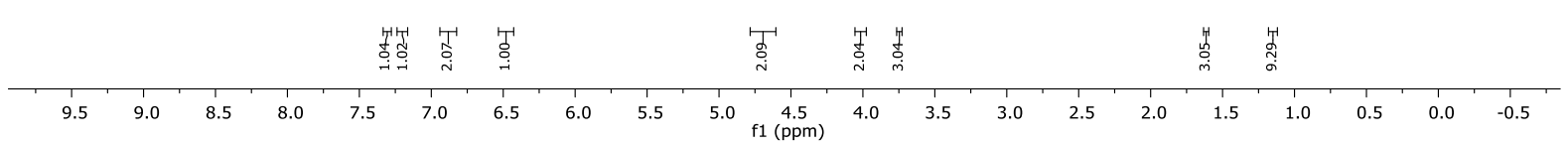

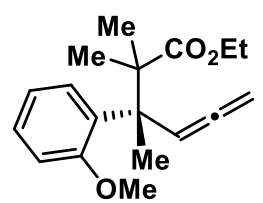

3n

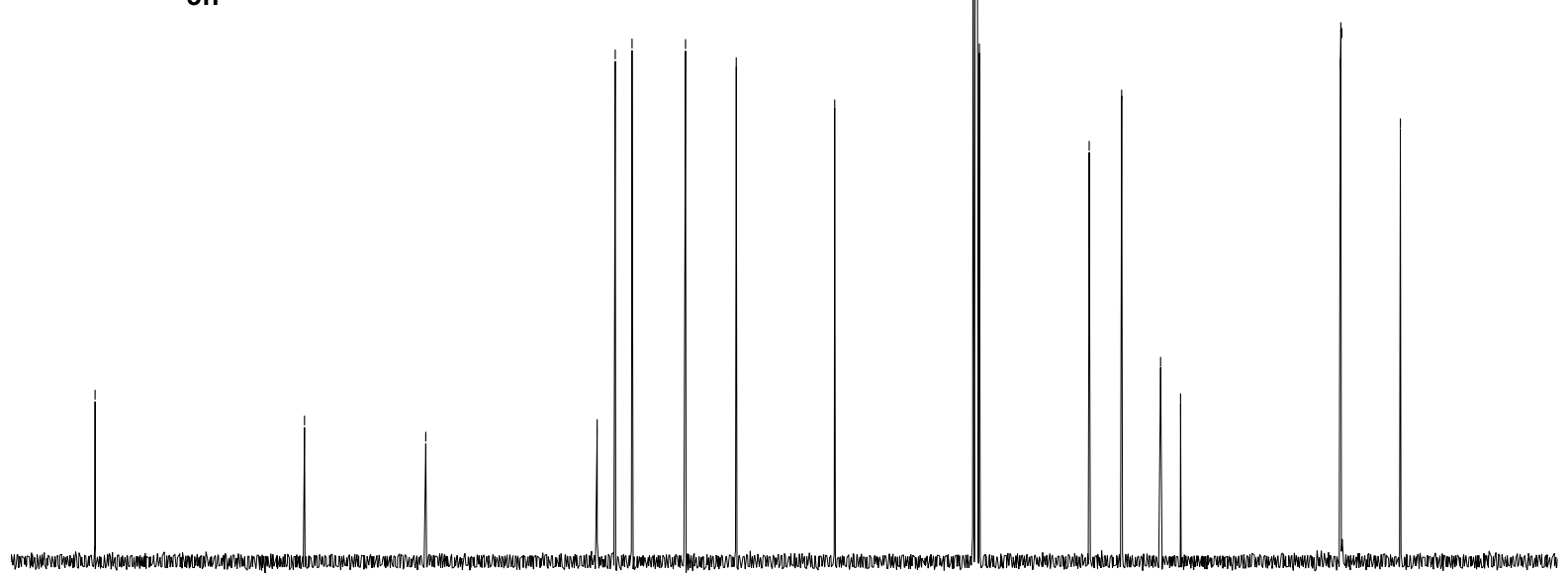

$\begin{array}{llllllllllll}210 & 200 & 190 & 180 & 170 & 160 & 150 & 140 & 130 & 120 & \underset{f}{110}(\mathrm{ppm}) & 100\end{array}$ 

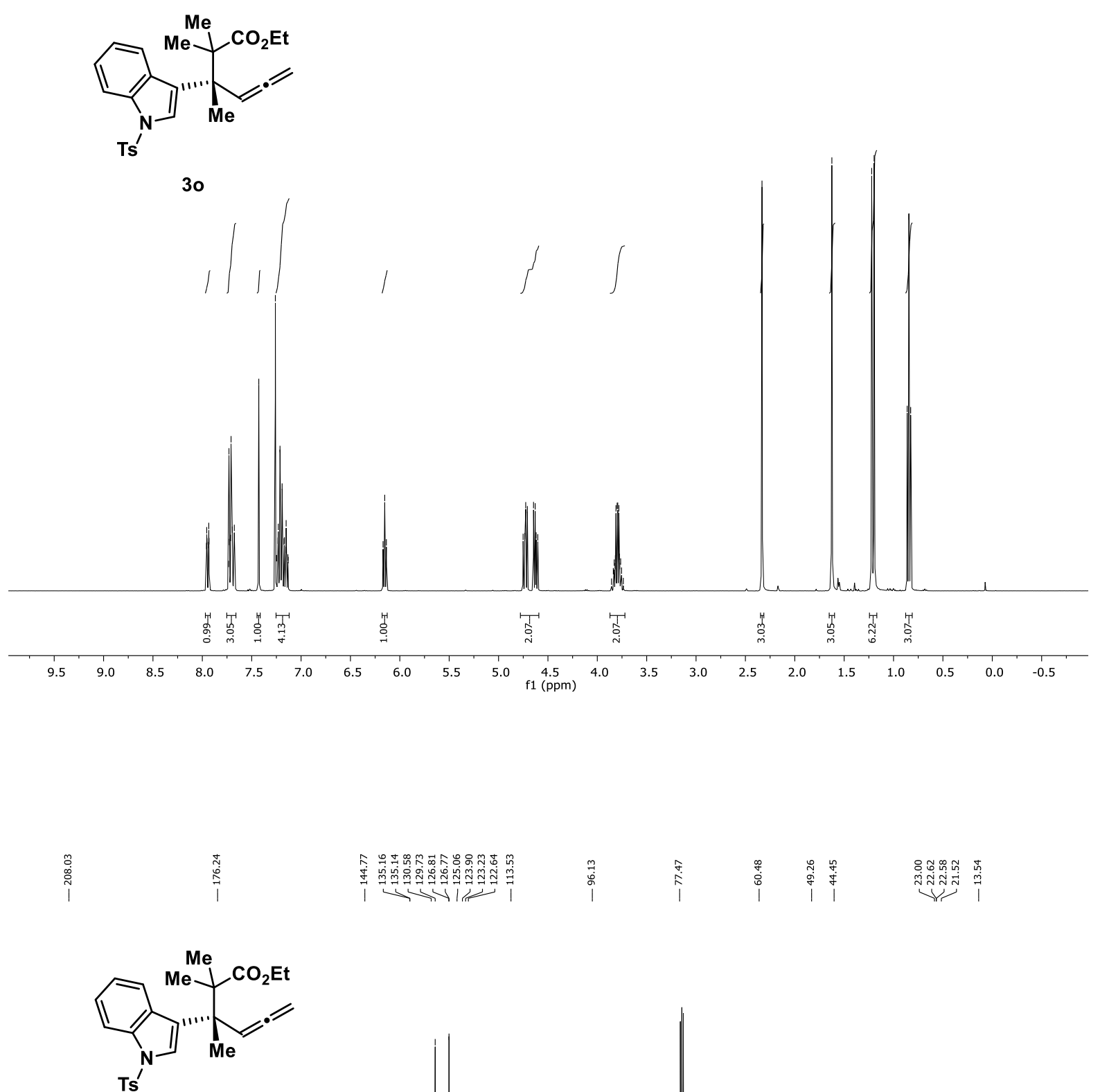

30

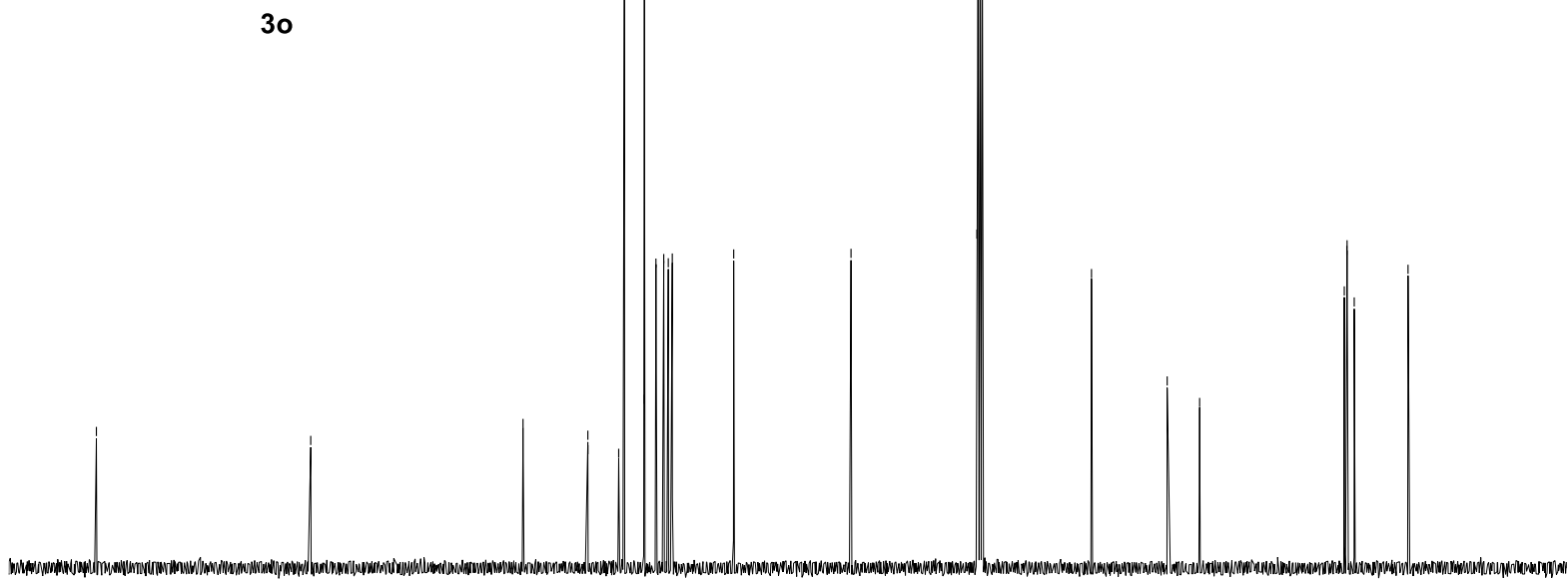

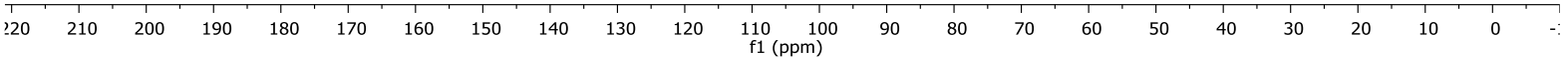




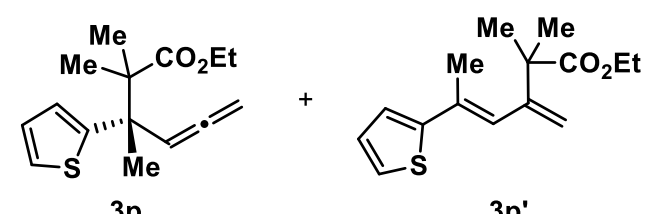

$3 p$

$3 p^{\prime}$

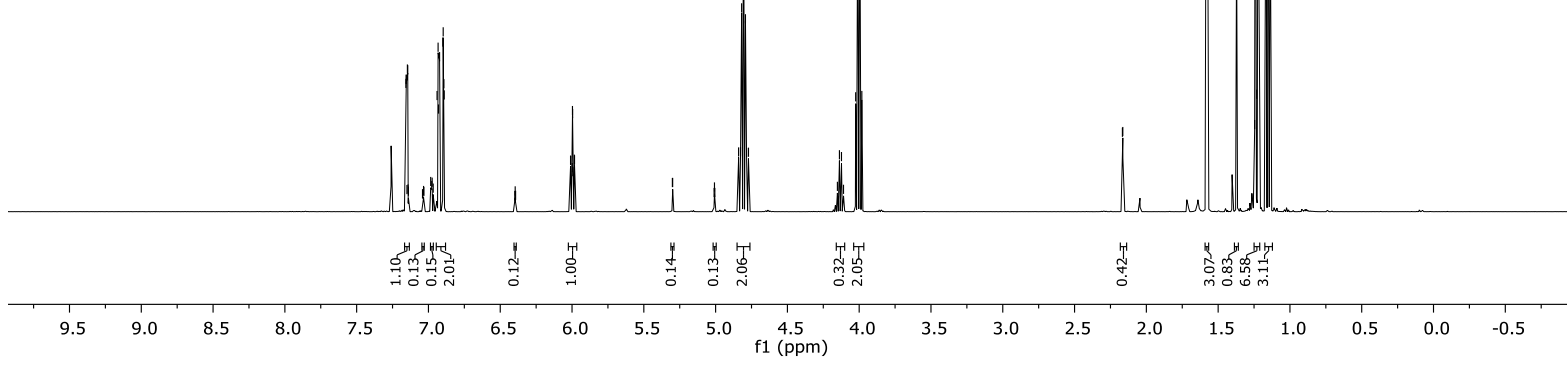

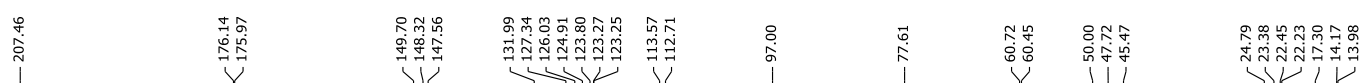
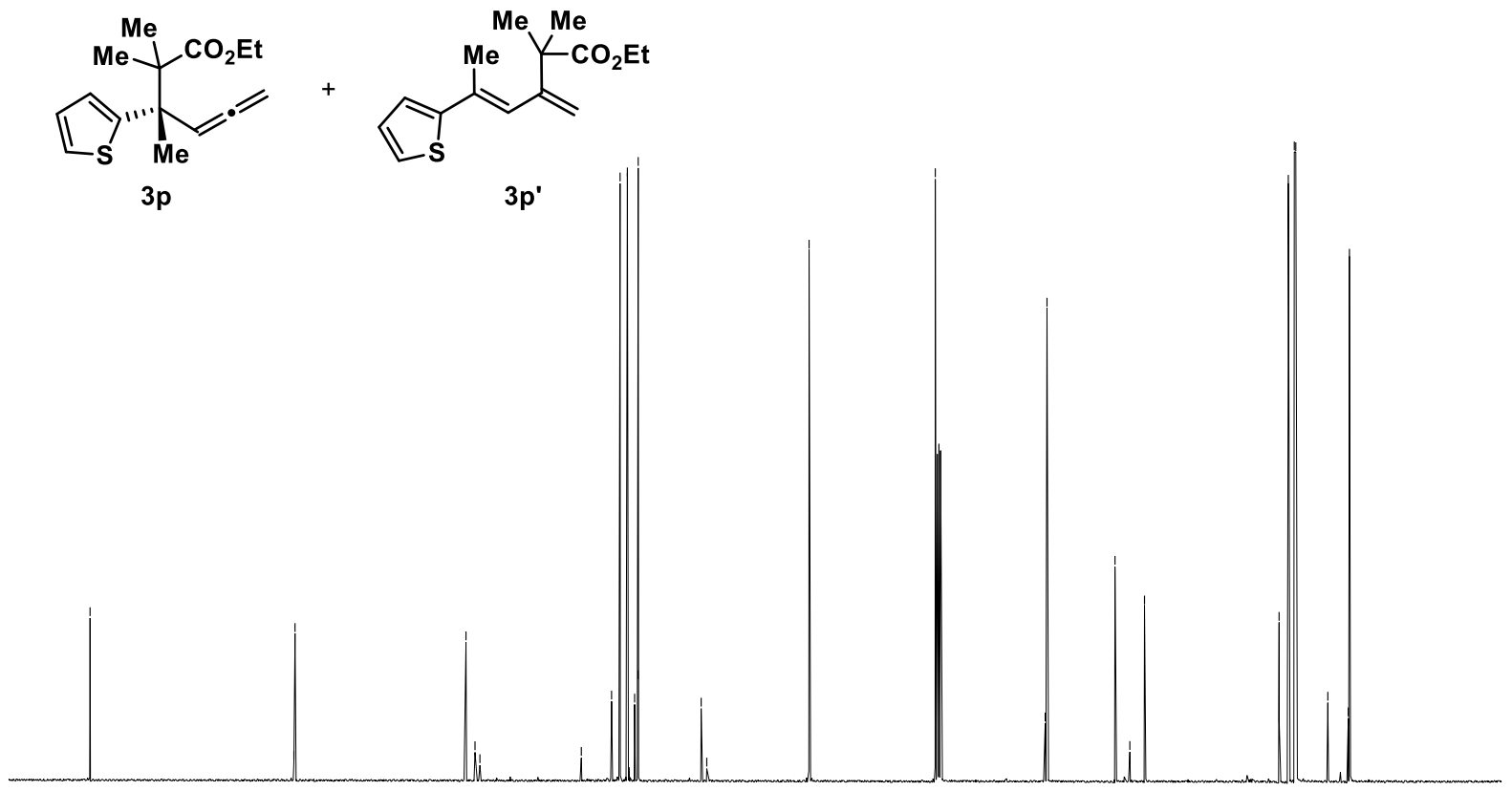

$\begin{array}{lllllllllllllllllllllll}1 & 210 & 200 & 190 & 180 & 170 & 160 & 150 & 140 & 130 & 120 & 110 & 100 & 90 & 80 & 70 & 60 & 50 & 40 & 30 & 20 & 10 & 0\end{array}$ 


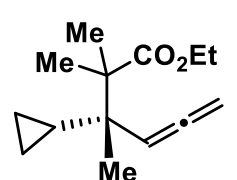

$3 q$

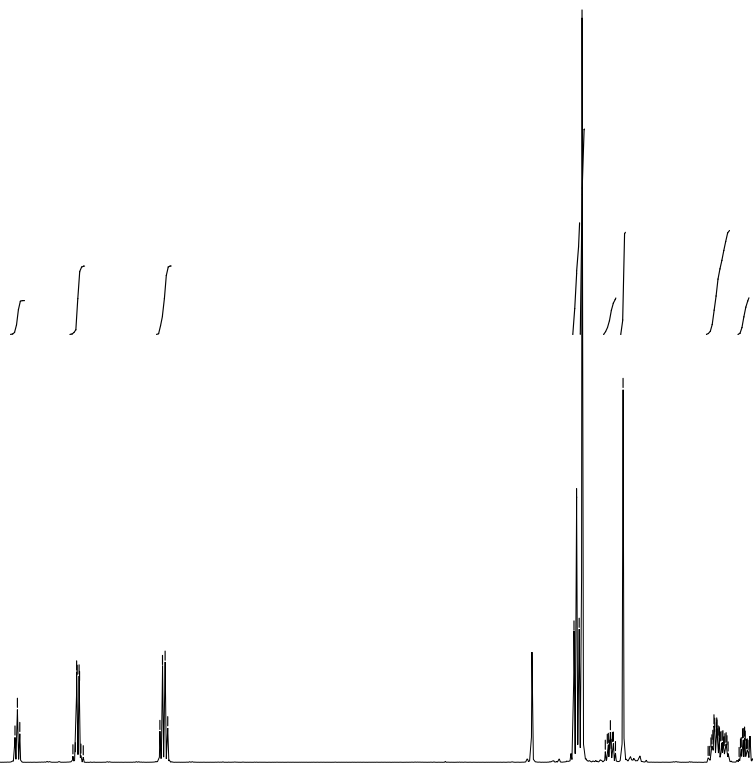

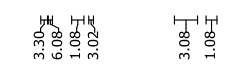

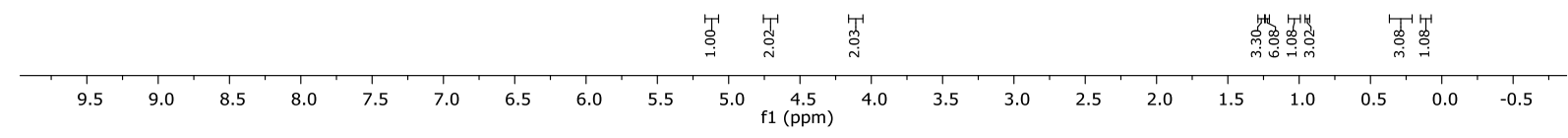

$\stackrel{\substack{0 \\ 0}}{\substack{0 \\ \stackrel{0}{0}}}$

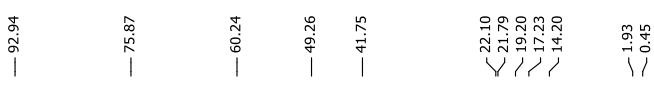

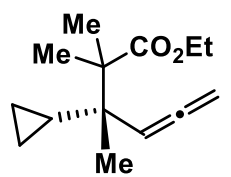

$3 q$

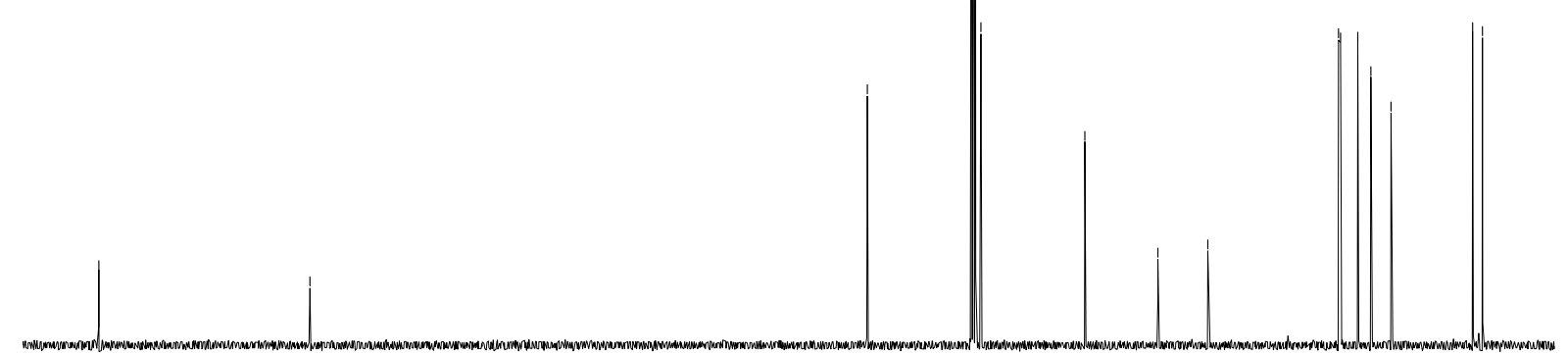

$\begin{array}{lllllllllllllllllllllllll}1 & 200 & 190 & 180 & 170 & 160 & 150 & 140 & 130 & 120 & 110 & 100 & 90 & 80 & 70 & 60 & 50 & 40 & 30 & 20 & 10 & 0 & -1\end{array}$ 


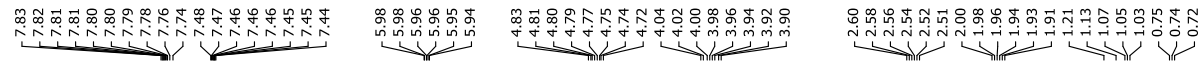

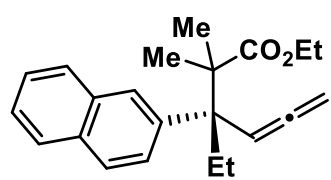

3s
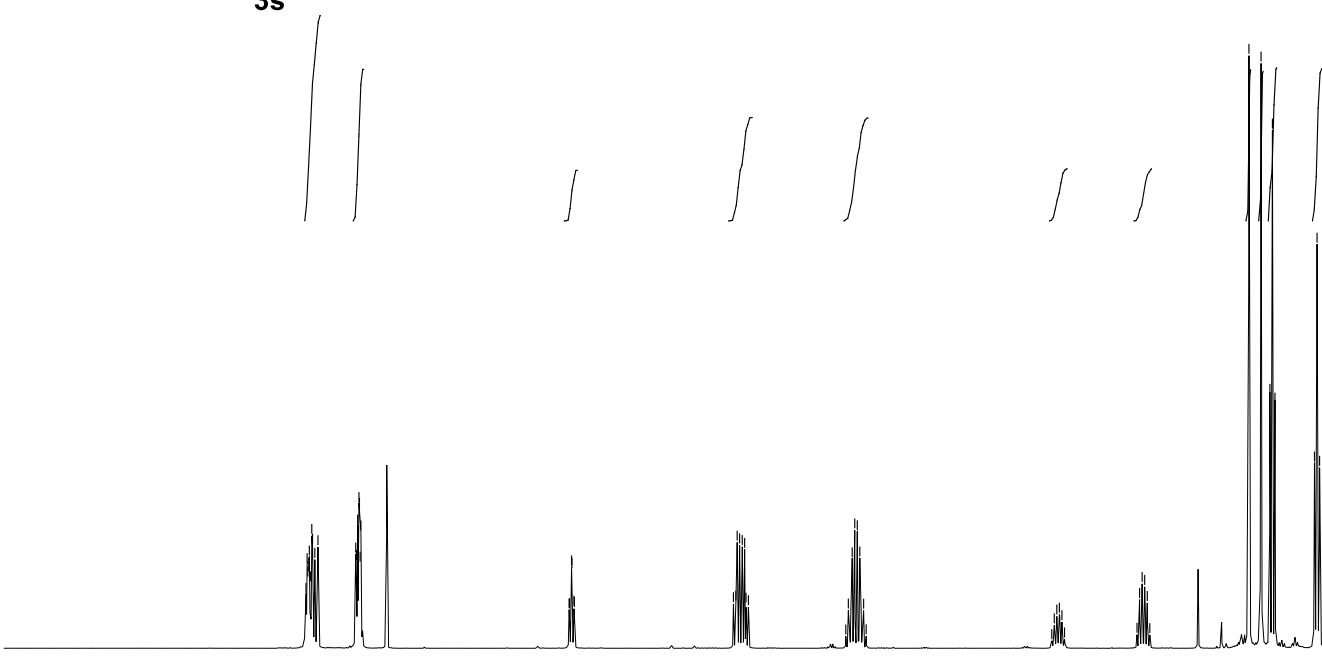

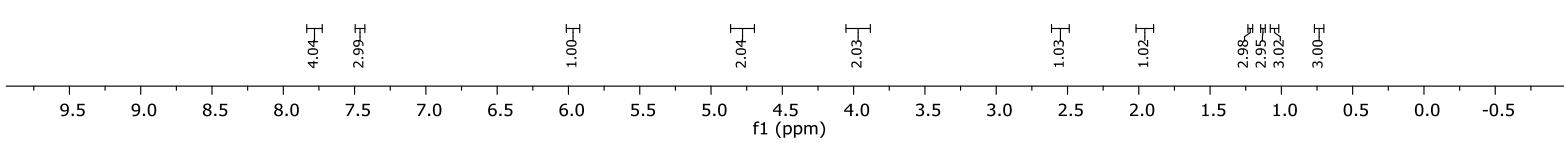

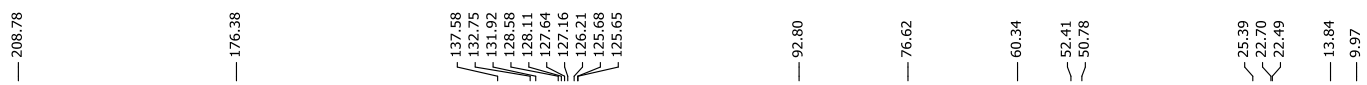<smiles>C=C=C[C@](CC)(c1ccc2ccccc2c1)C(C)(C)OCC</smiles>

3s

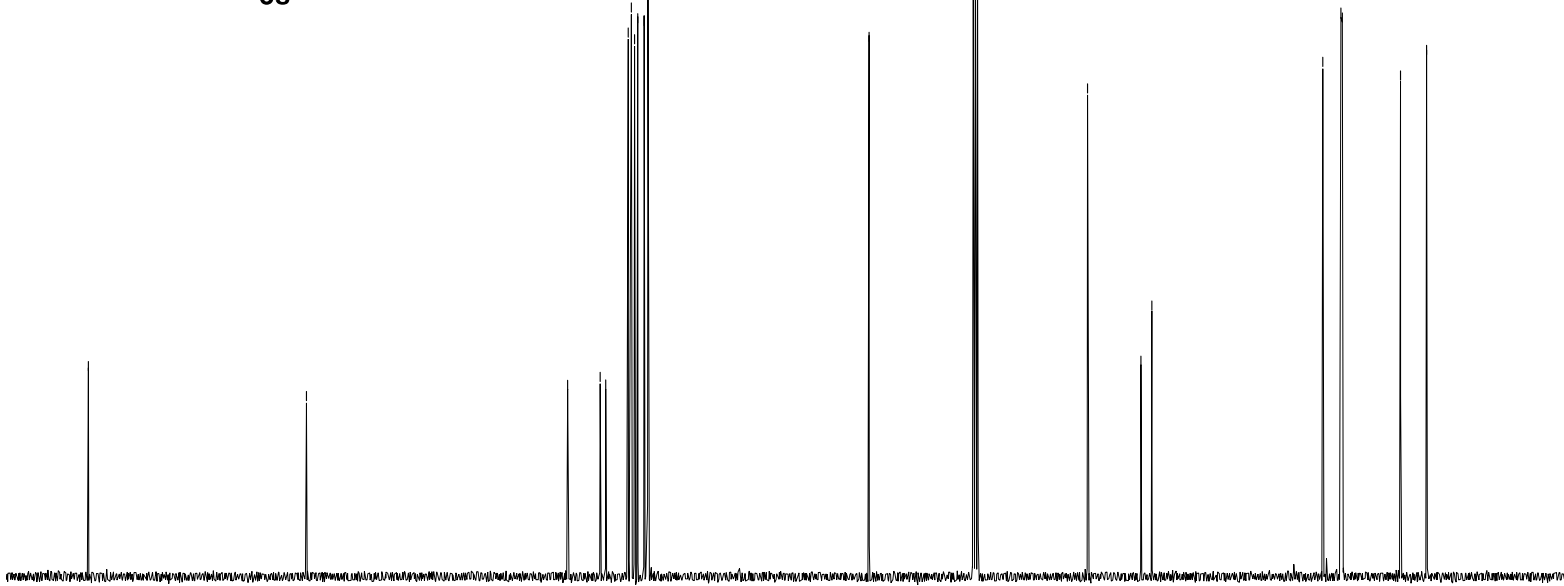

$\begin{array}{lllllllllllllllllllllllll}120 & 210 & 200 & 190 & 180 & 170 & 160 & 150 & 140 & 130 & 120 & 110 & 100 & 90 & 80 & 70 & 60 & 50 & 40 & 30 & 20 & 10 & 0 & -1\end{array}$ 


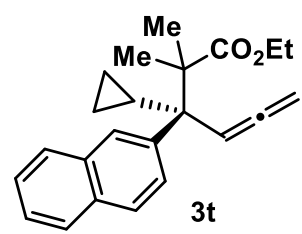

\|\|
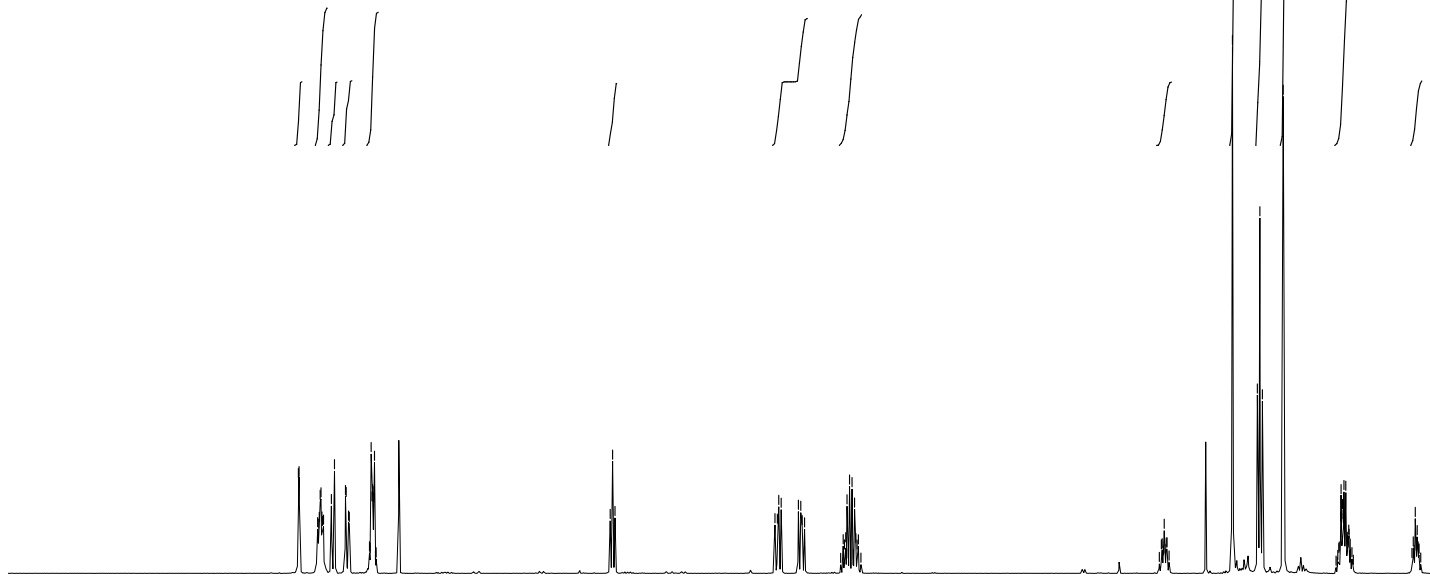

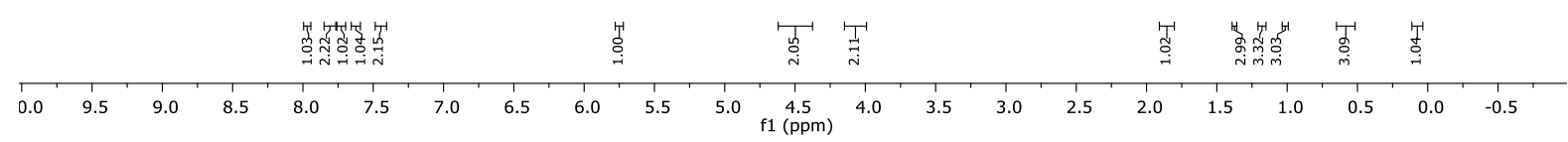
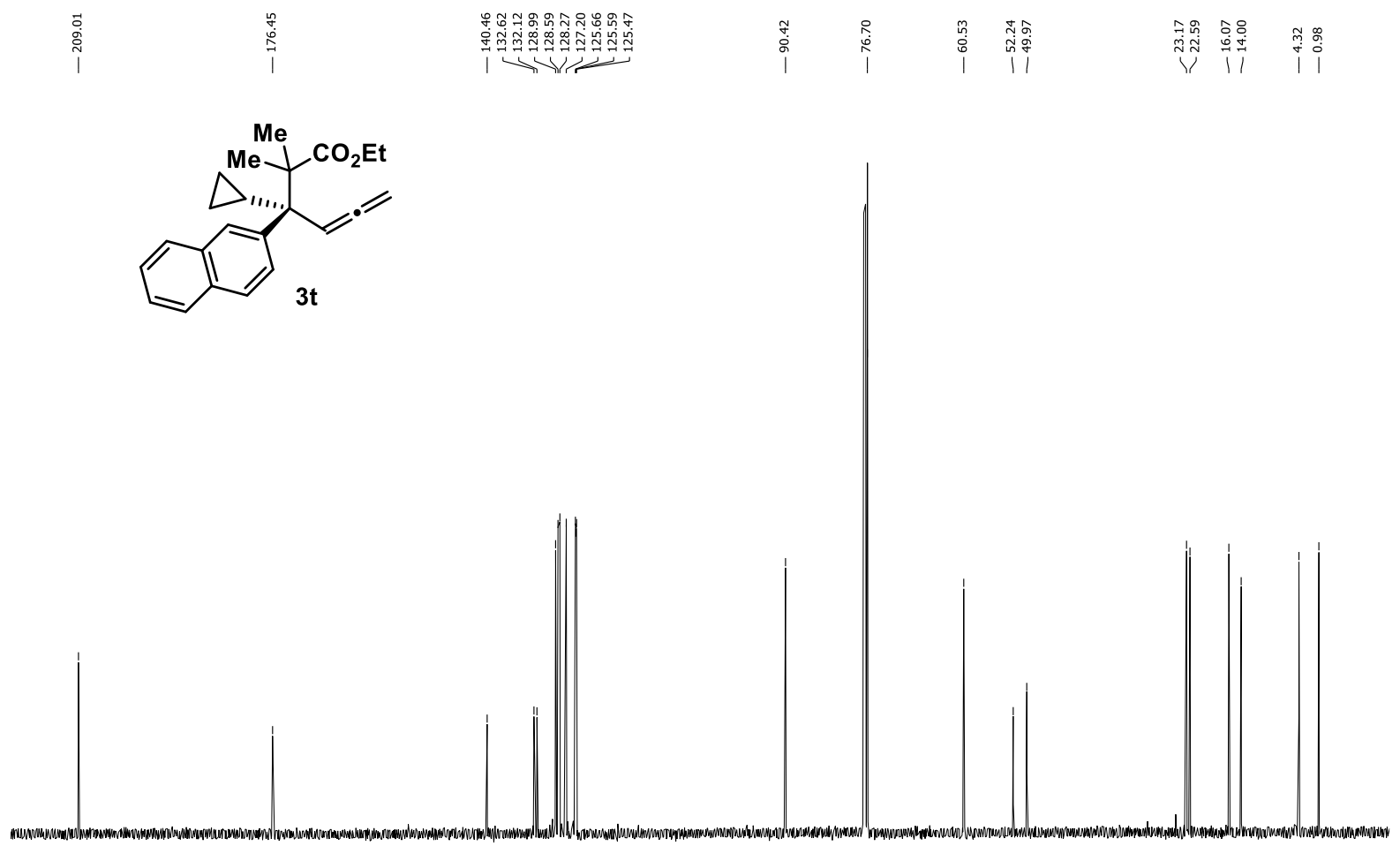

\begin{tabular}{llllllllllllllllllllllll}
\hline 20 & 210 & 200 & 190 & 180 & 170 & 160 & 150 & 140 & 130 & 120 & 110 & 100 & 90 & 80 & 70 & 60 & 50 & 40 & 30 & 20 & 10 & 0 & -1
\end{tabular} 


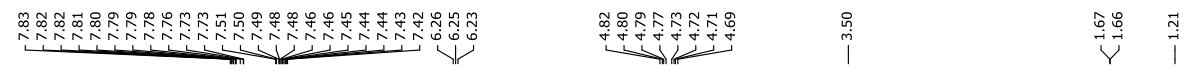

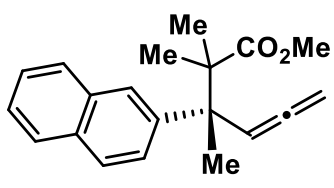

$3 u$
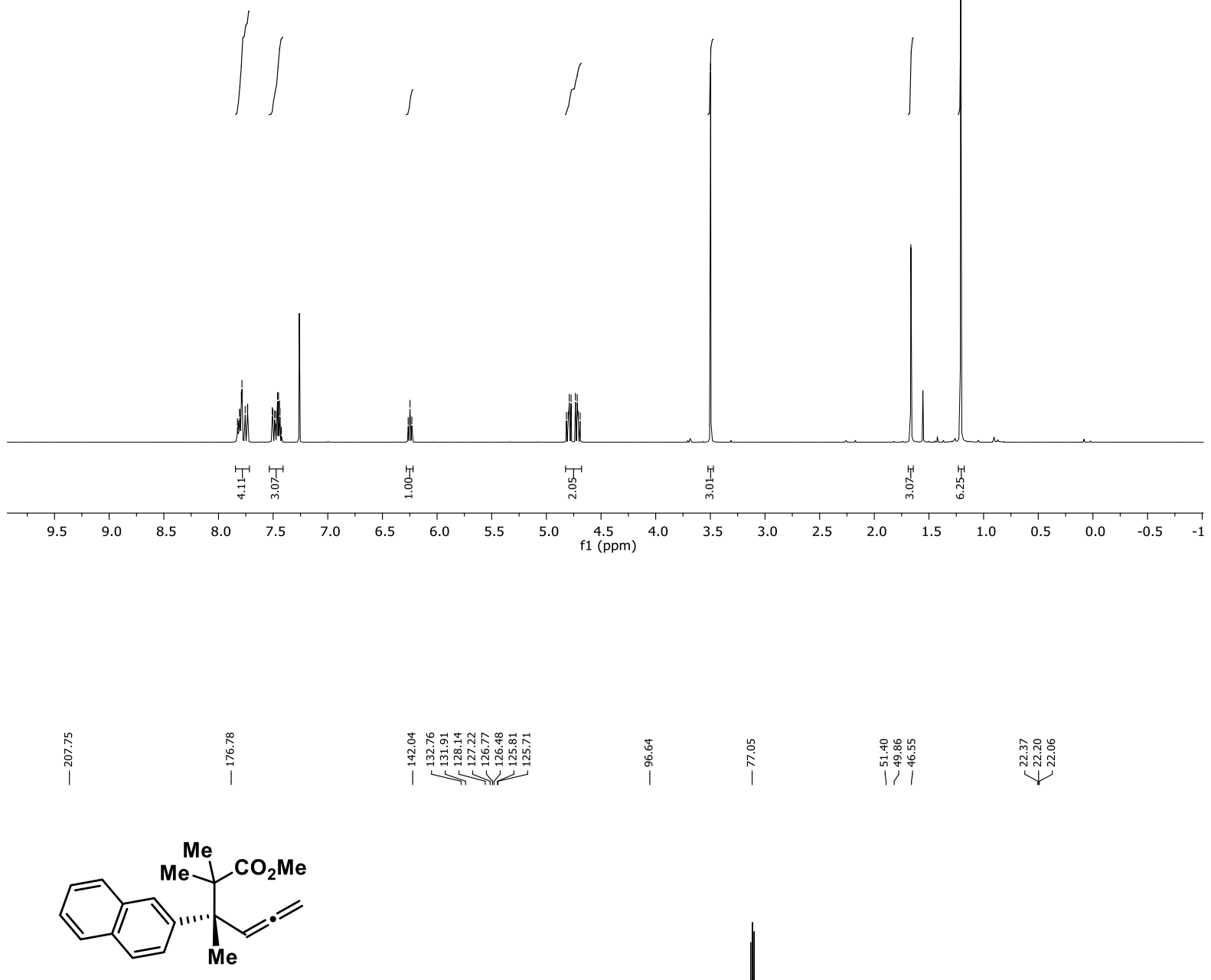

$3 u$

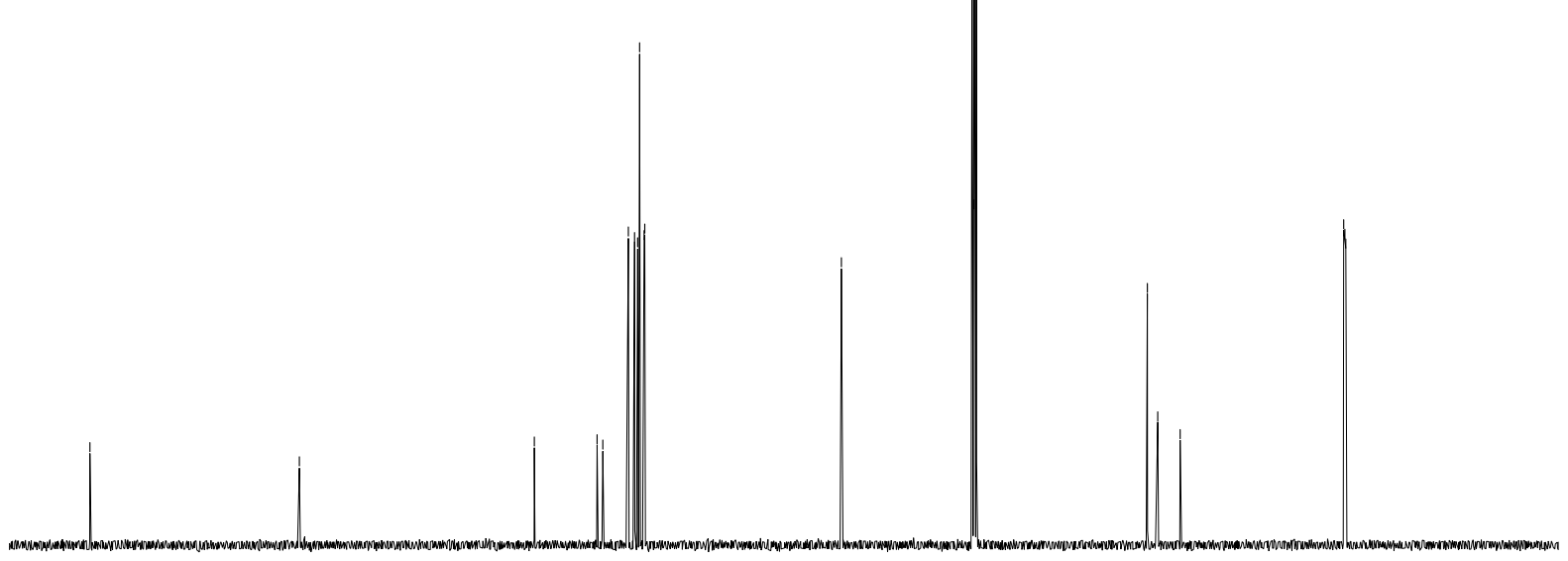

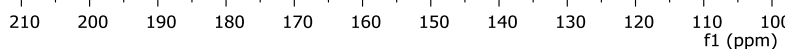




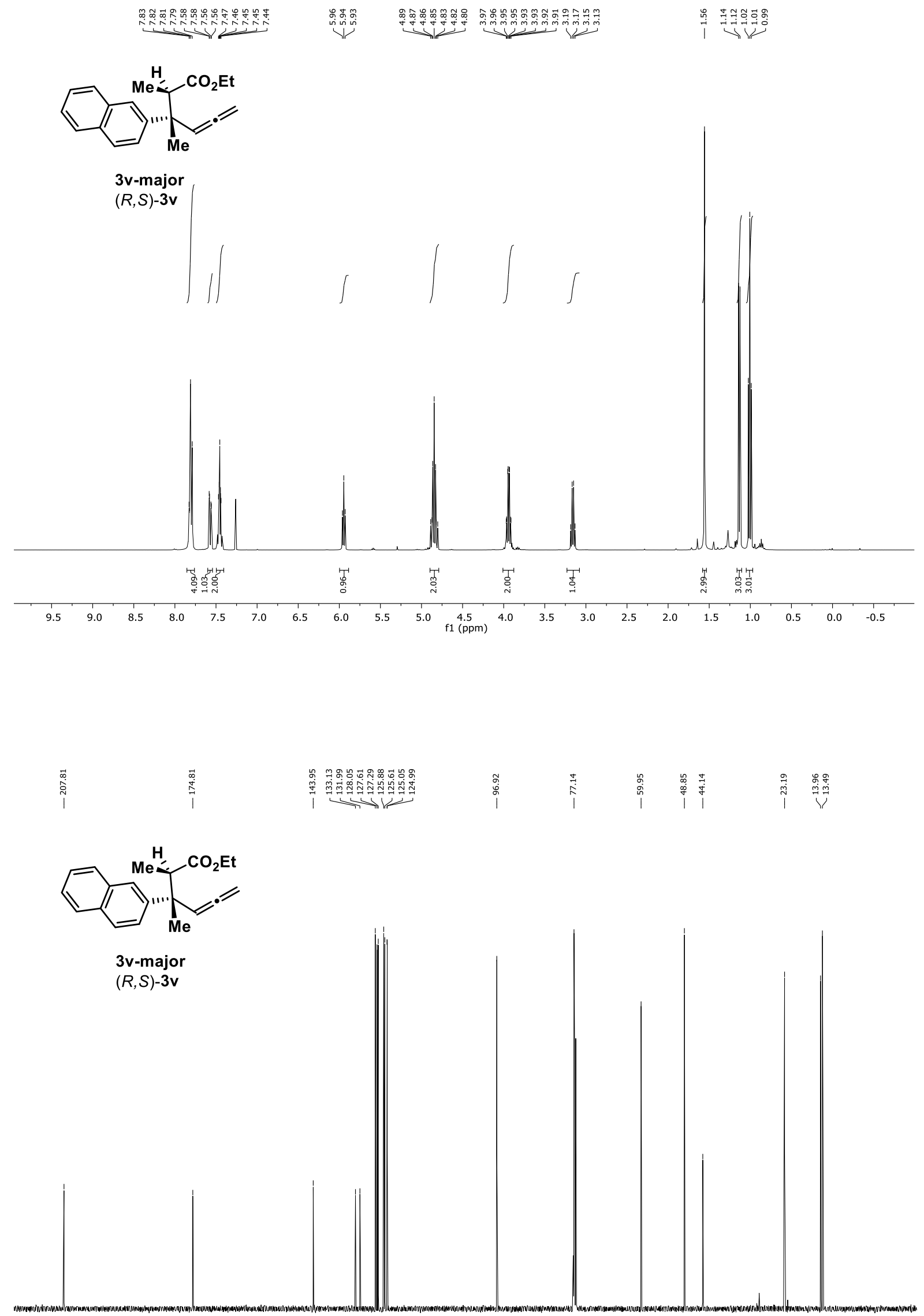

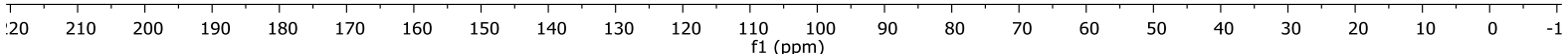



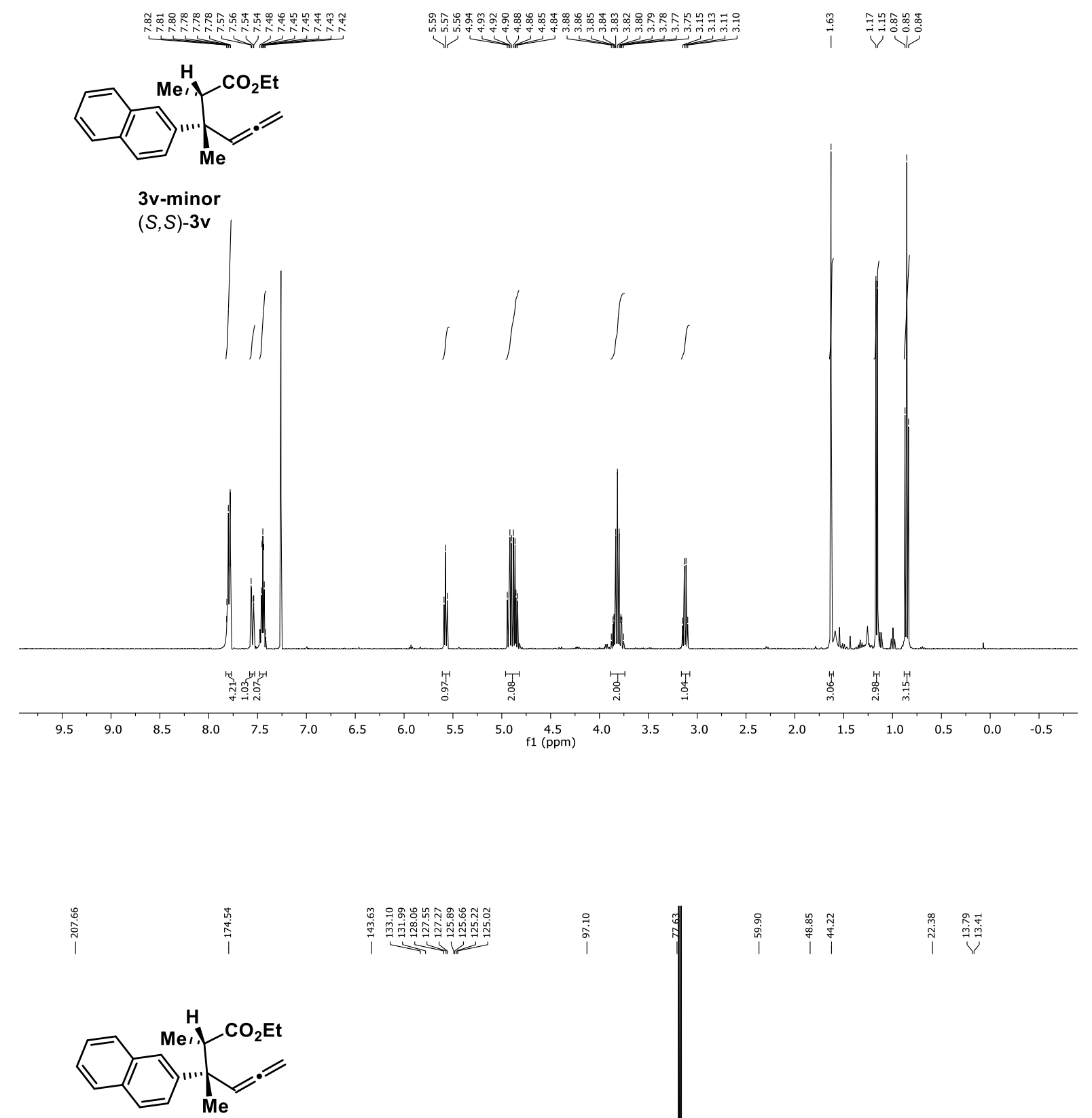

|

3v-minor

$(S, S)-3 v$

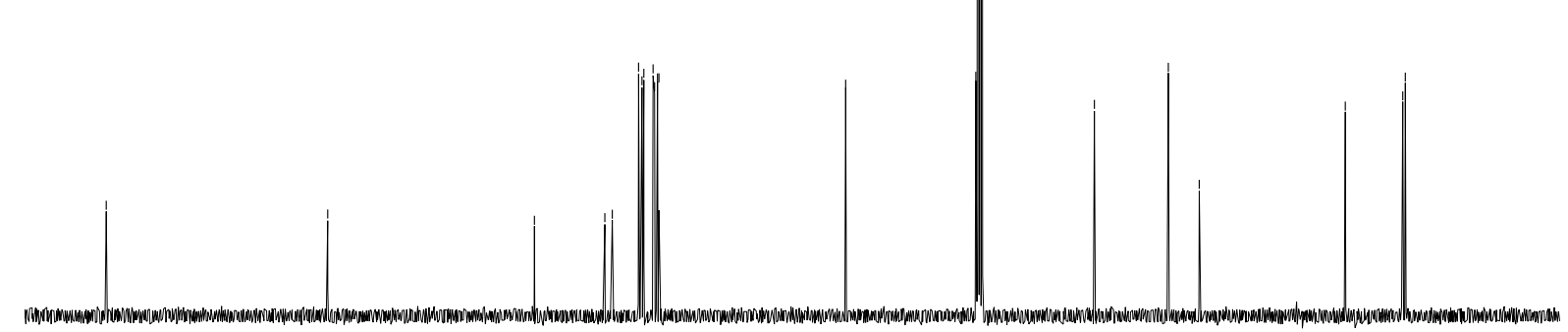

$\begin{array}{lllllllllll}210 & 200 & 190 & 180 & 170 & 160 & 150 & 140 & 130 & 120 & 110 \\ \mathrm{f} 1(\mathrm{ppm})\end{array}$ 

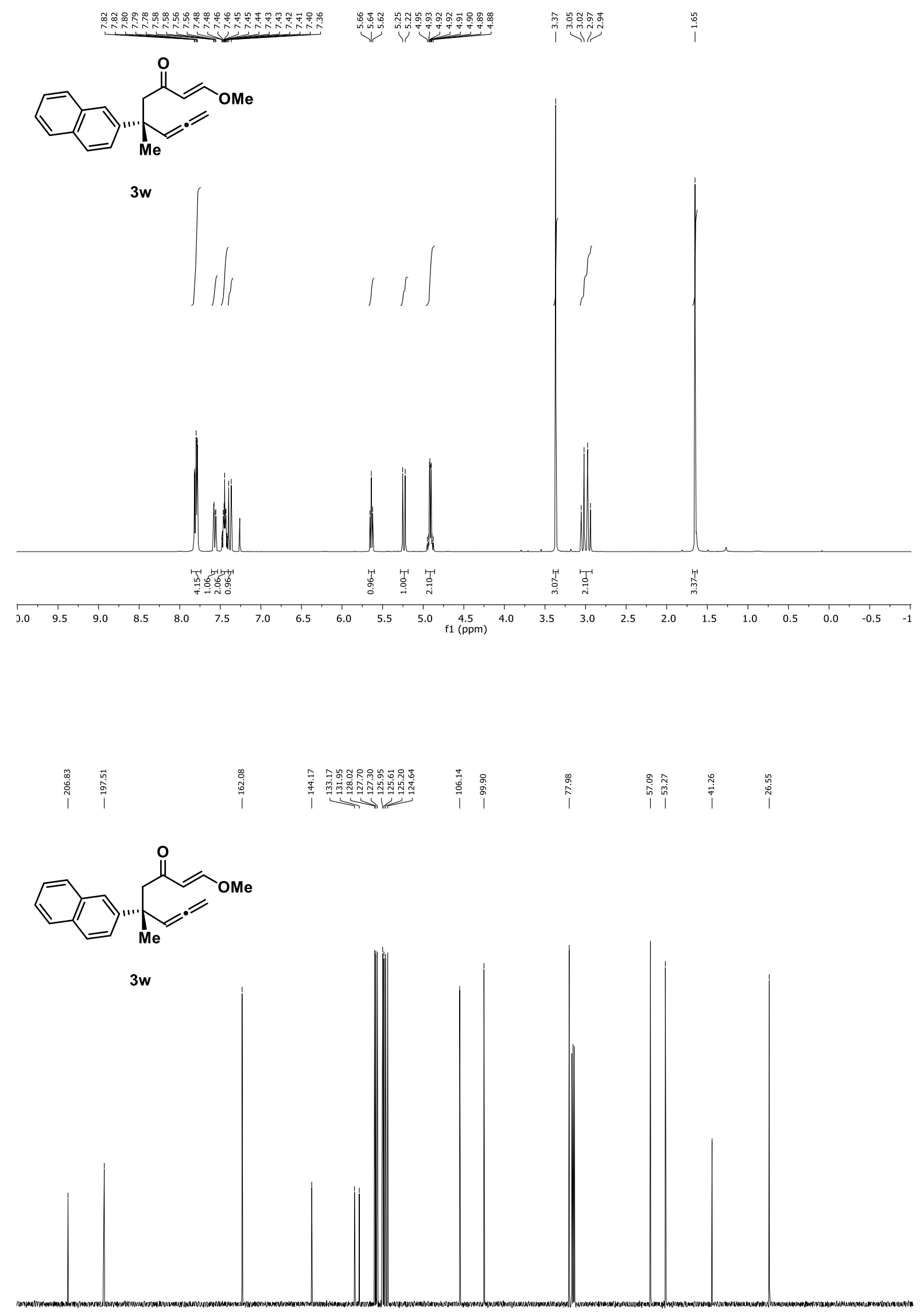

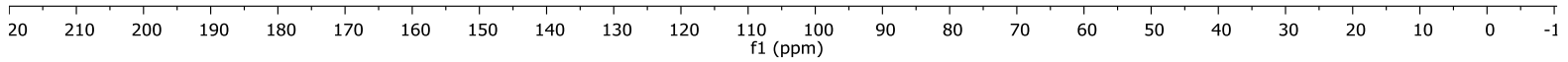




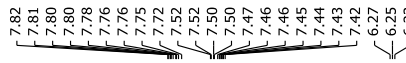

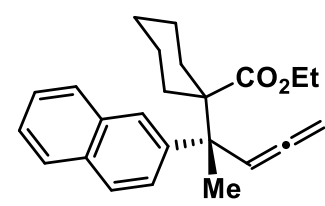

$3 \mathbf{x}$<smiles>CCCCC</smiles>
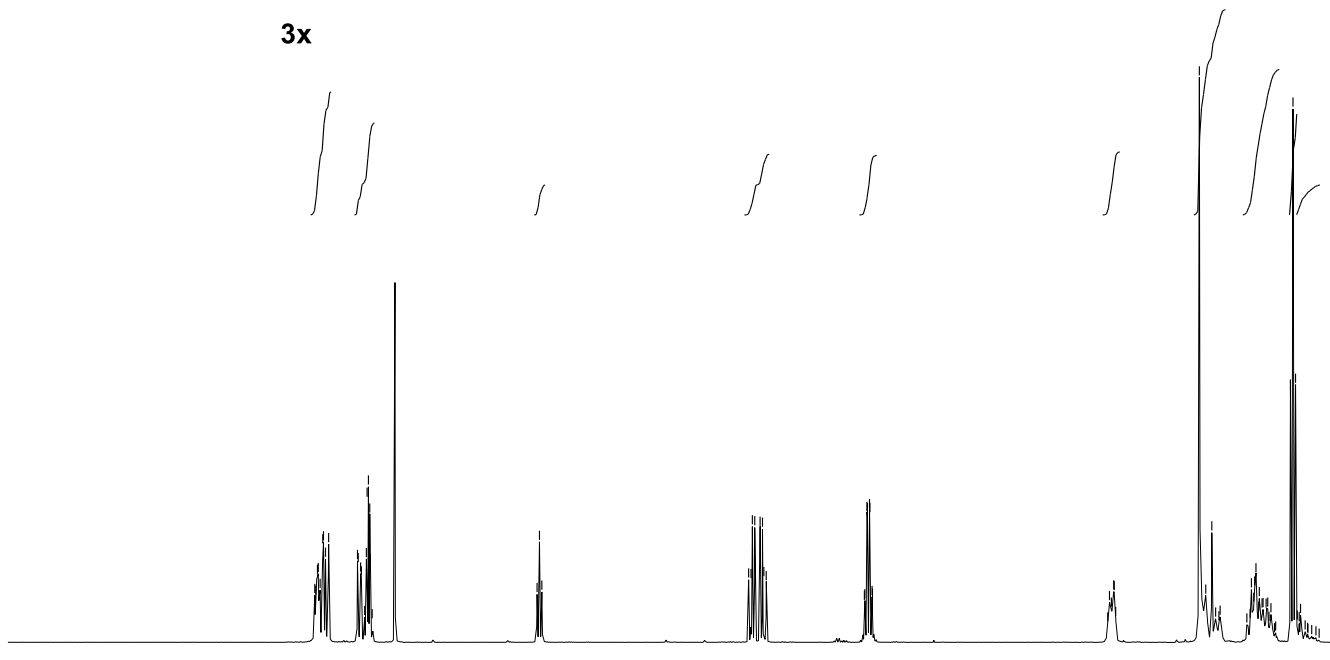

\begin{tabular}{|c|c|c|c|c|c|c|c|c|c|c|c|c|c|c|c|c|c|c|c|c|}
\hline & & & & 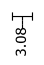 & & ț & & & & लू & 旿 & & & $\stackrel{T}{T}$ & & $\begin{array}{l}\qquad \\
\substack{\infty \\
\infty \\
0}\end{array}$ & 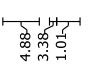 & & & \\
\hline 9.5 & 9.0 & 8.5 & 8.0 & 7.5 & 7.0 & 6.5 & 6.0 & 5.5 & 5.0 & $\begin{array}{c}4.5 \\
\text { f1 }(p p m)\end{array}$ & 4.0 & 3.5 & 3.0 & 2.5 & 2.0 & 1.5 & 1.0 & 0.5 & 0.0 & -0.5 \\
\hline
\end{tabular}

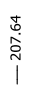
웅 |<smiles>C=C=C[C@](C)(c1ccc2ccccc2c1)C1(C(=O)OCC)CCCCC1</smiles>

$3 \mathbf{x}$

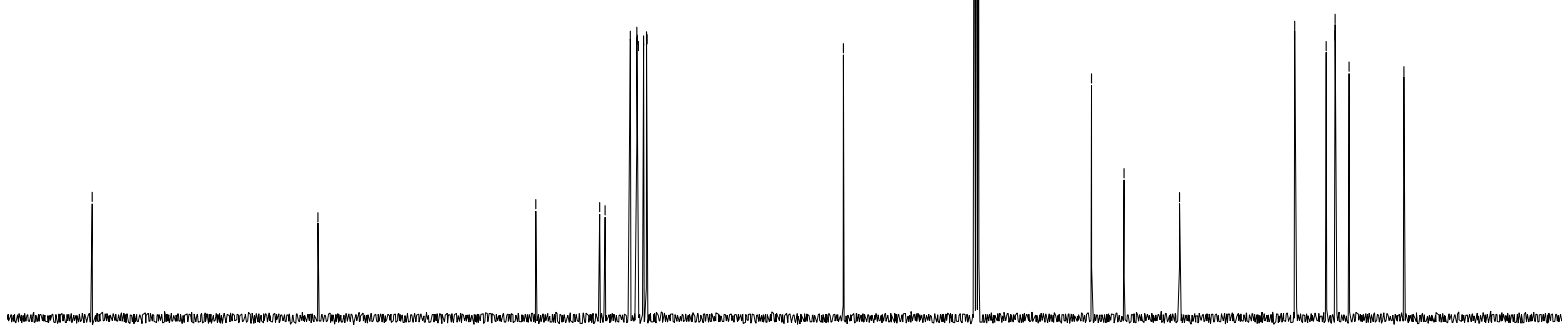

$\begin{array}{llllllllllll}20 & 210 & 200 & 190 & 180 & 170 & 160 & 150 & 140 & 130 & 120 & \substack{110 \\ \mathrm{f} 1(\mathrm{ppm})} \\ 100 & & & & & & \end{array}$ 

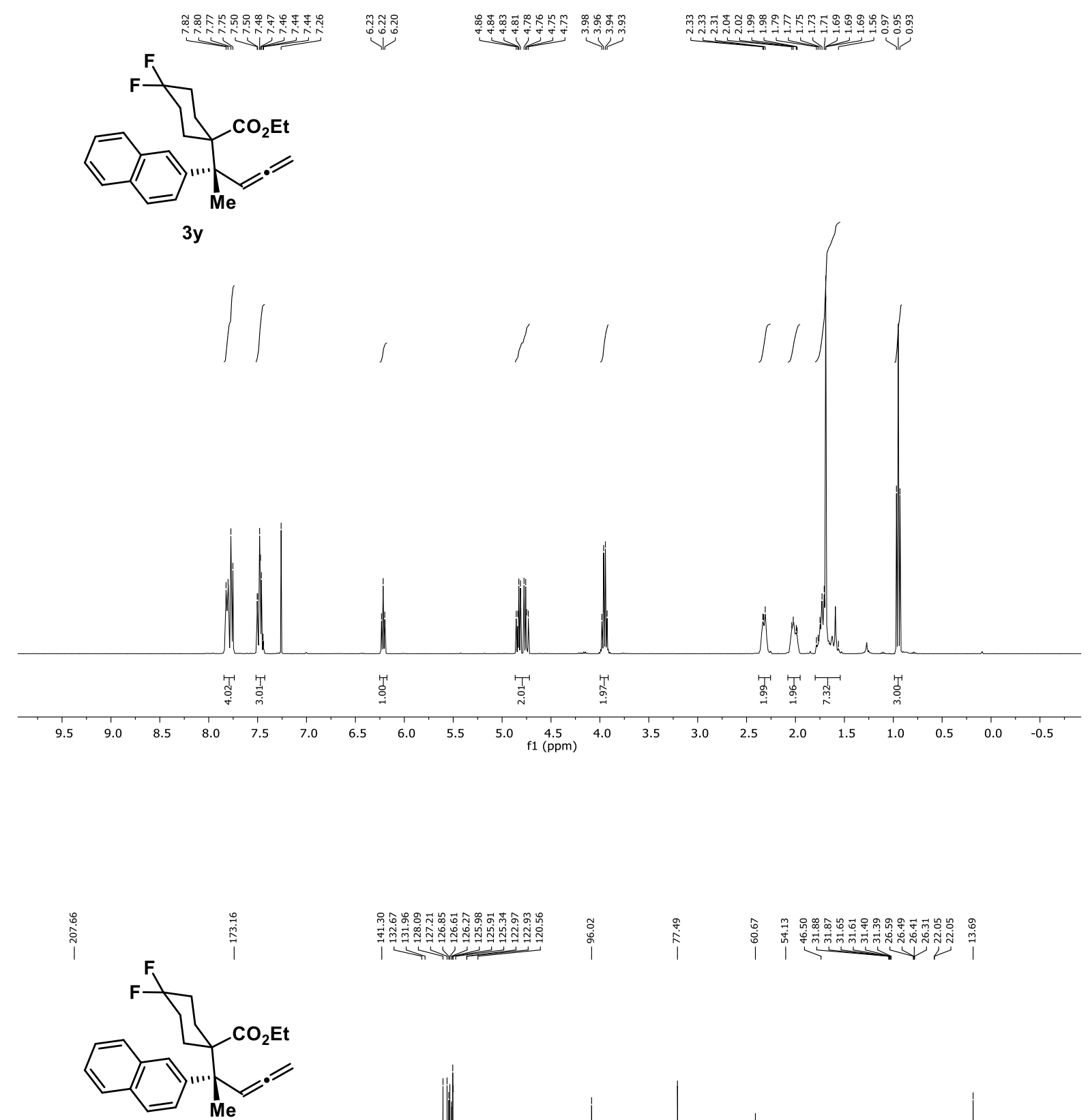

3y

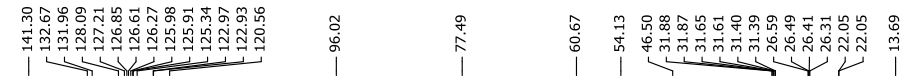

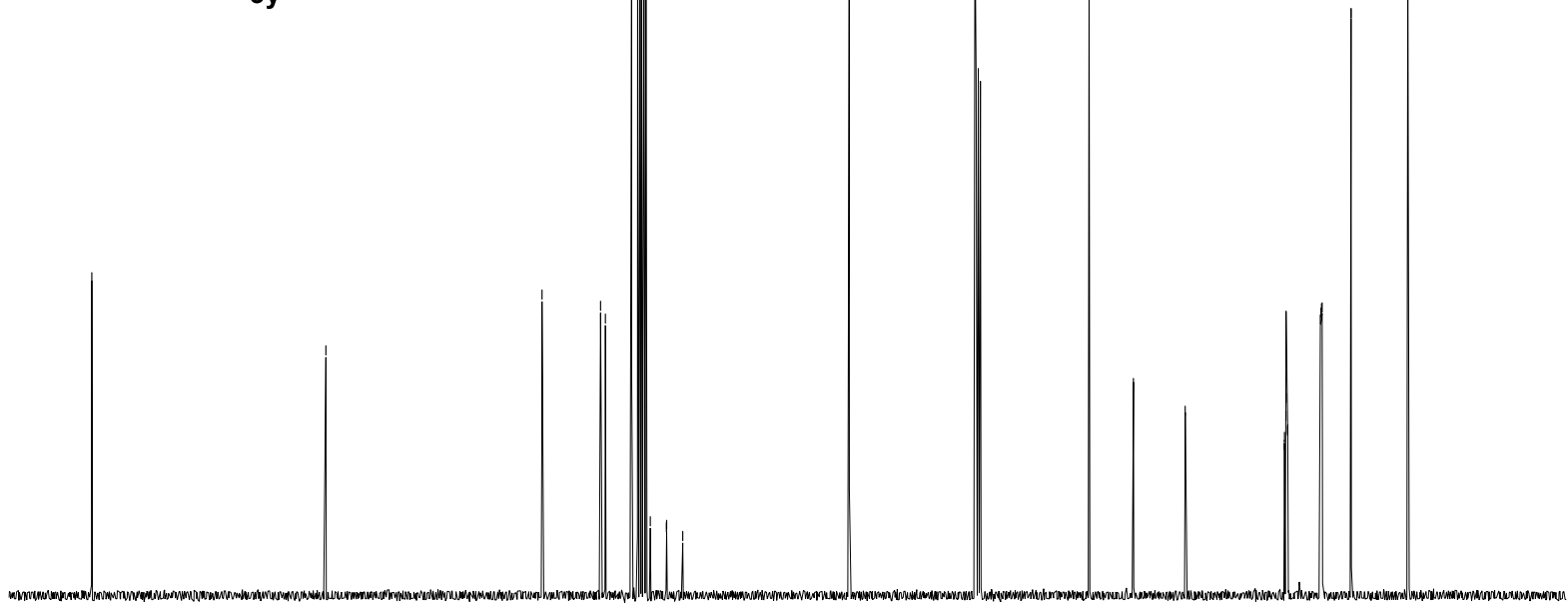

$\begin{array}{lllllllllll}210 & 200 & 190 & 180 & 170 & 160 & 150 & 140 & 130 & 120 & 110 \begin{array}{r}100 \\ \mathrm{f} 1(\mathrm{ppm})\end{array}\end{array}$ 


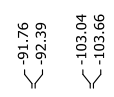
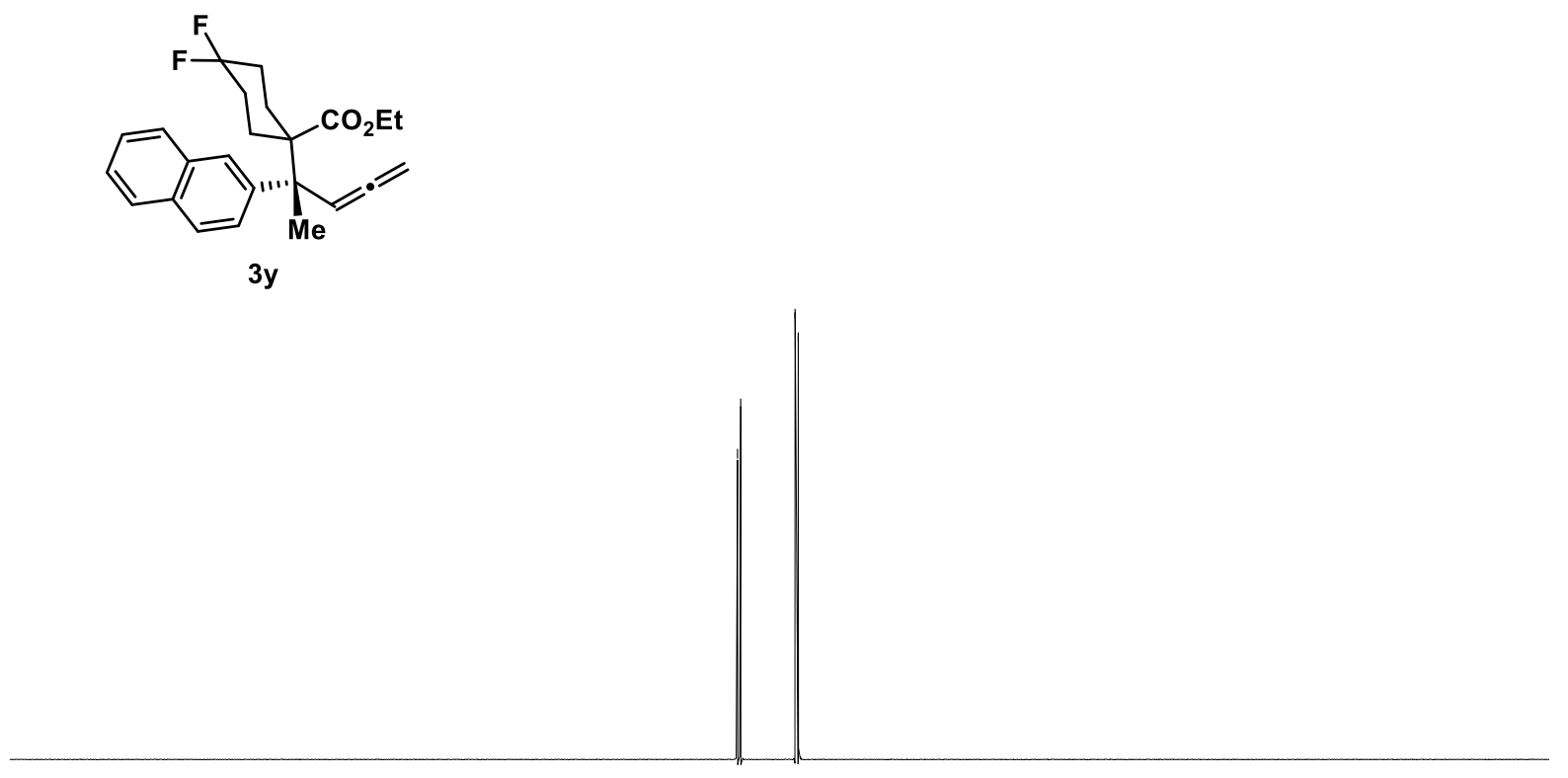

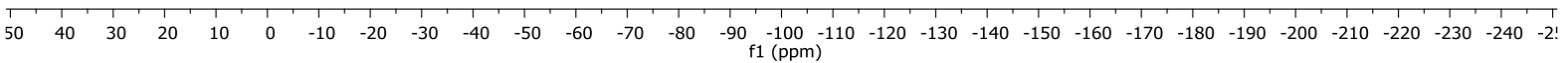


20.

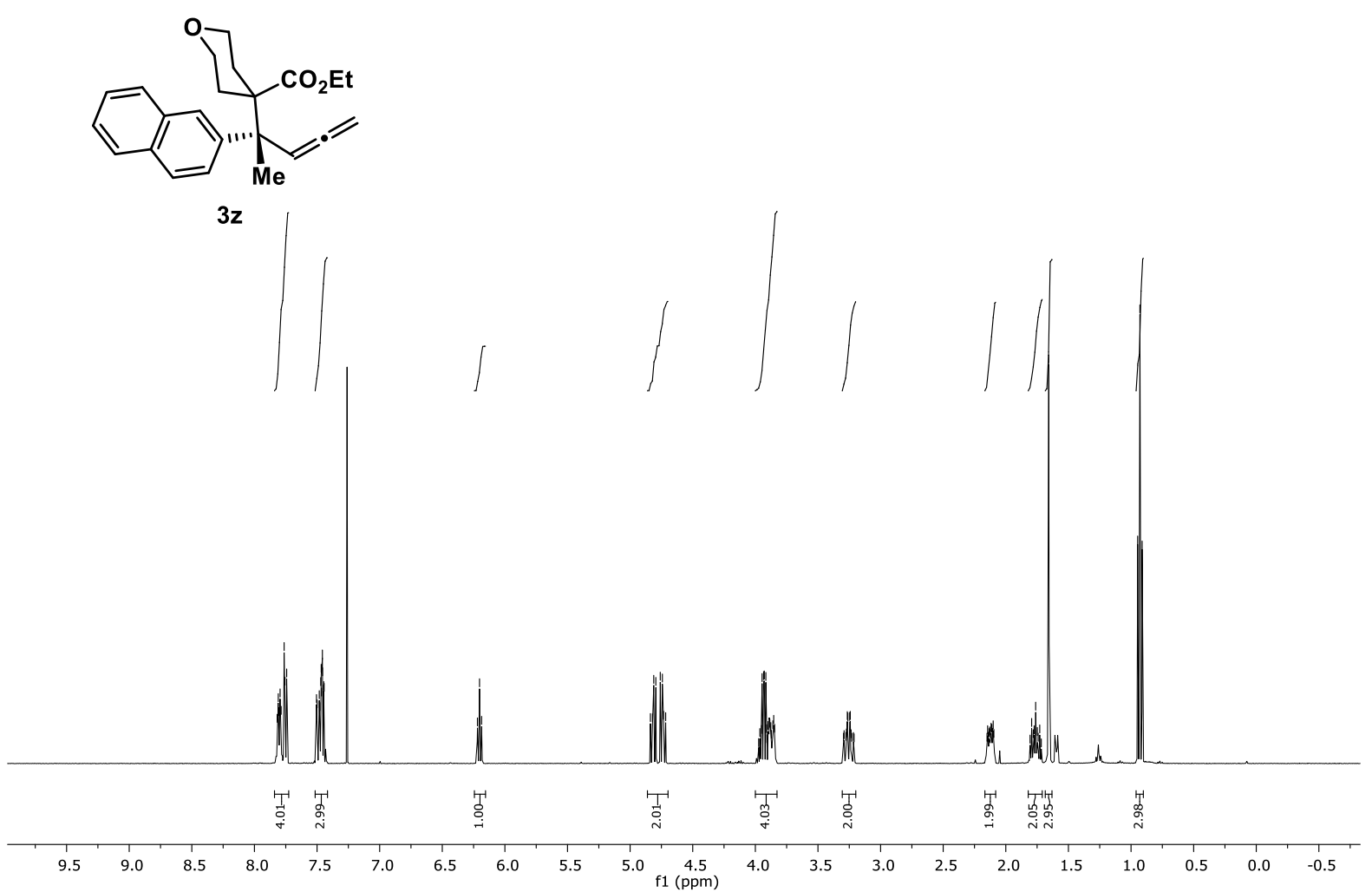

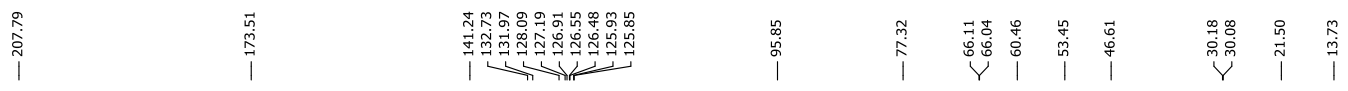

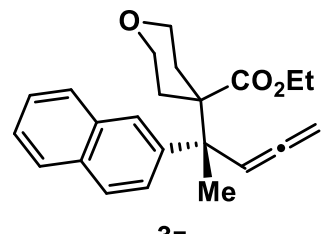

$3 z$

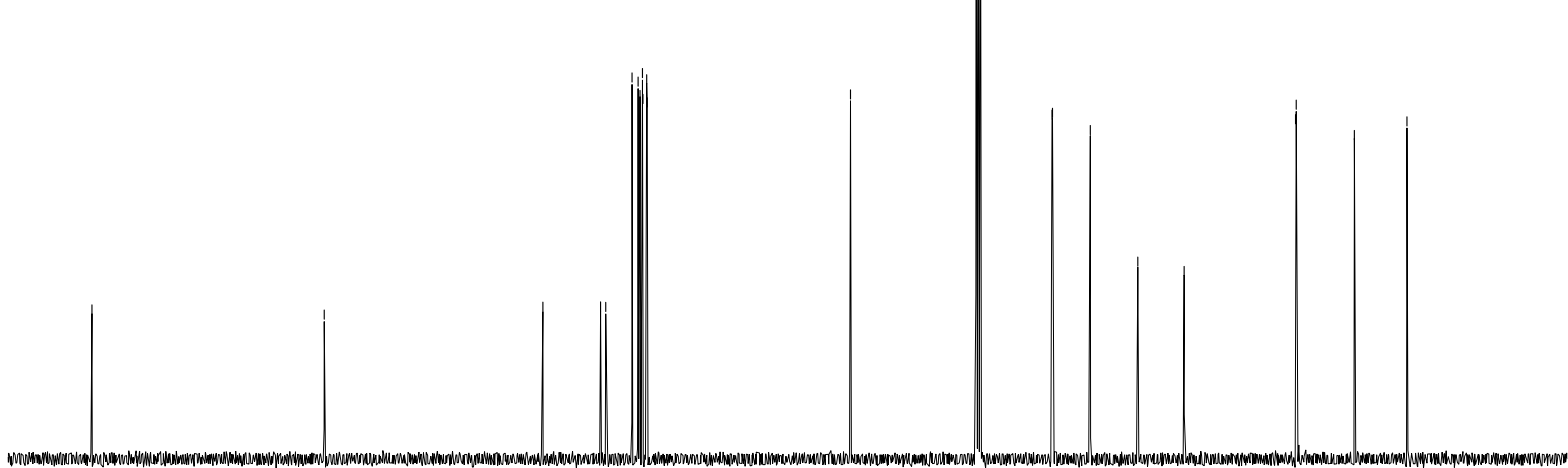

$\begin{array}{llllllllllll}20 & 210 & 200 & 190 & 180 & 170 & 160 & 150 & 140 & 130 & 120 & 110 \quad \begin{array}{l}100 \\ f 1(\mathrm{pmm})\end{array}\end{array}$ 


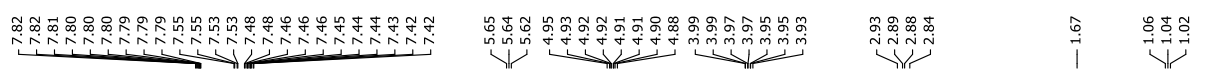

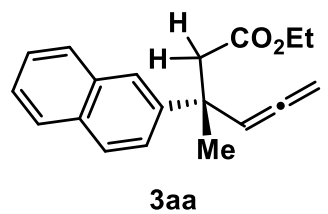

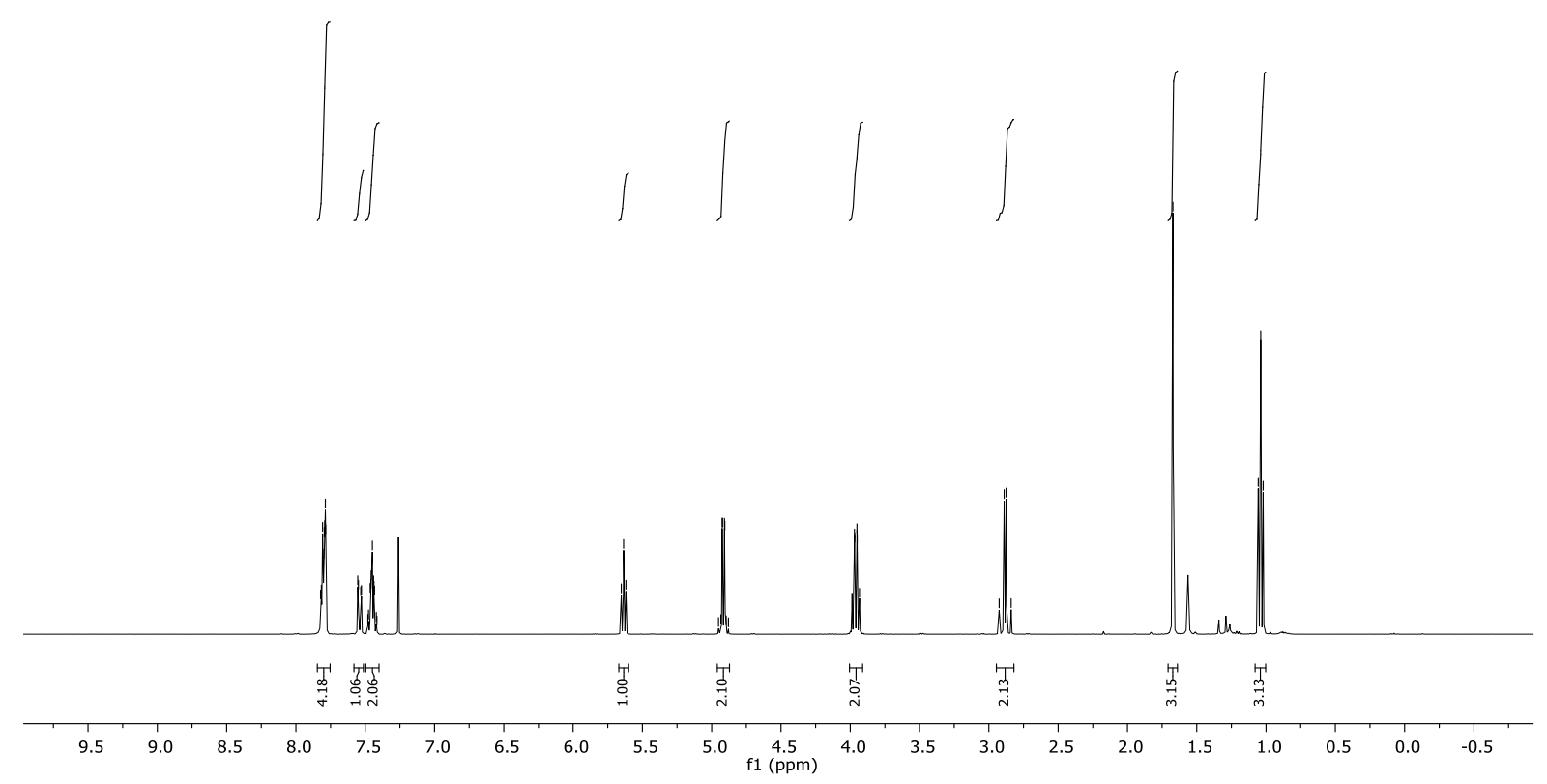

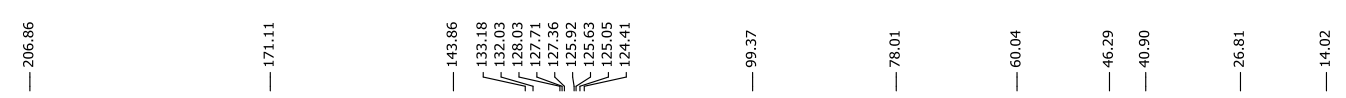

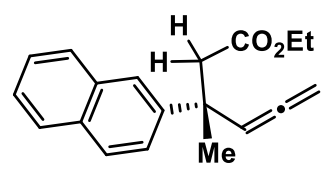

3 aa

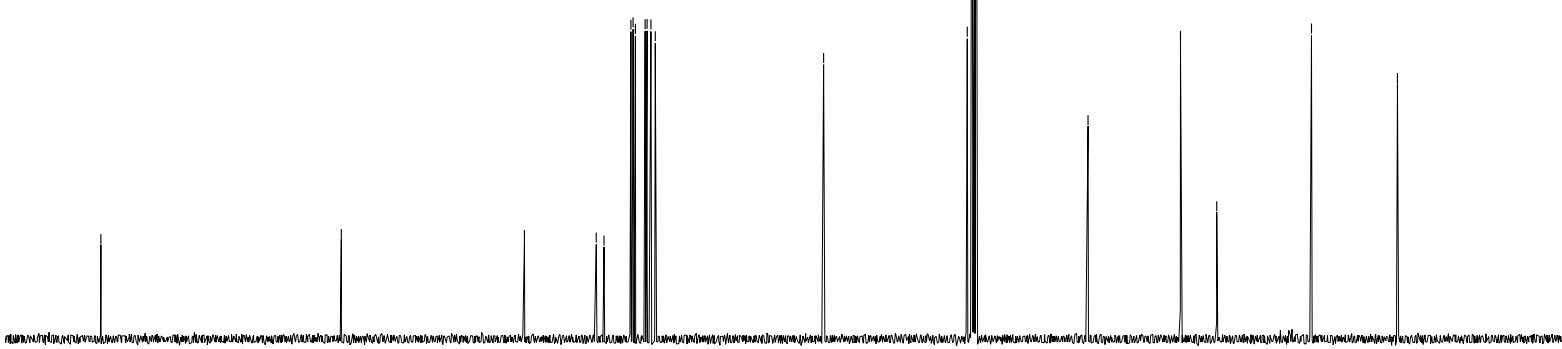

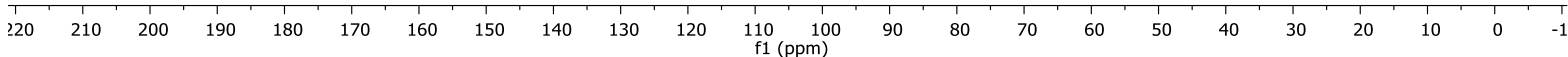




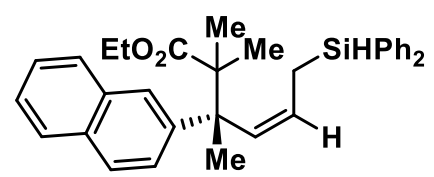

$(Z)-5$

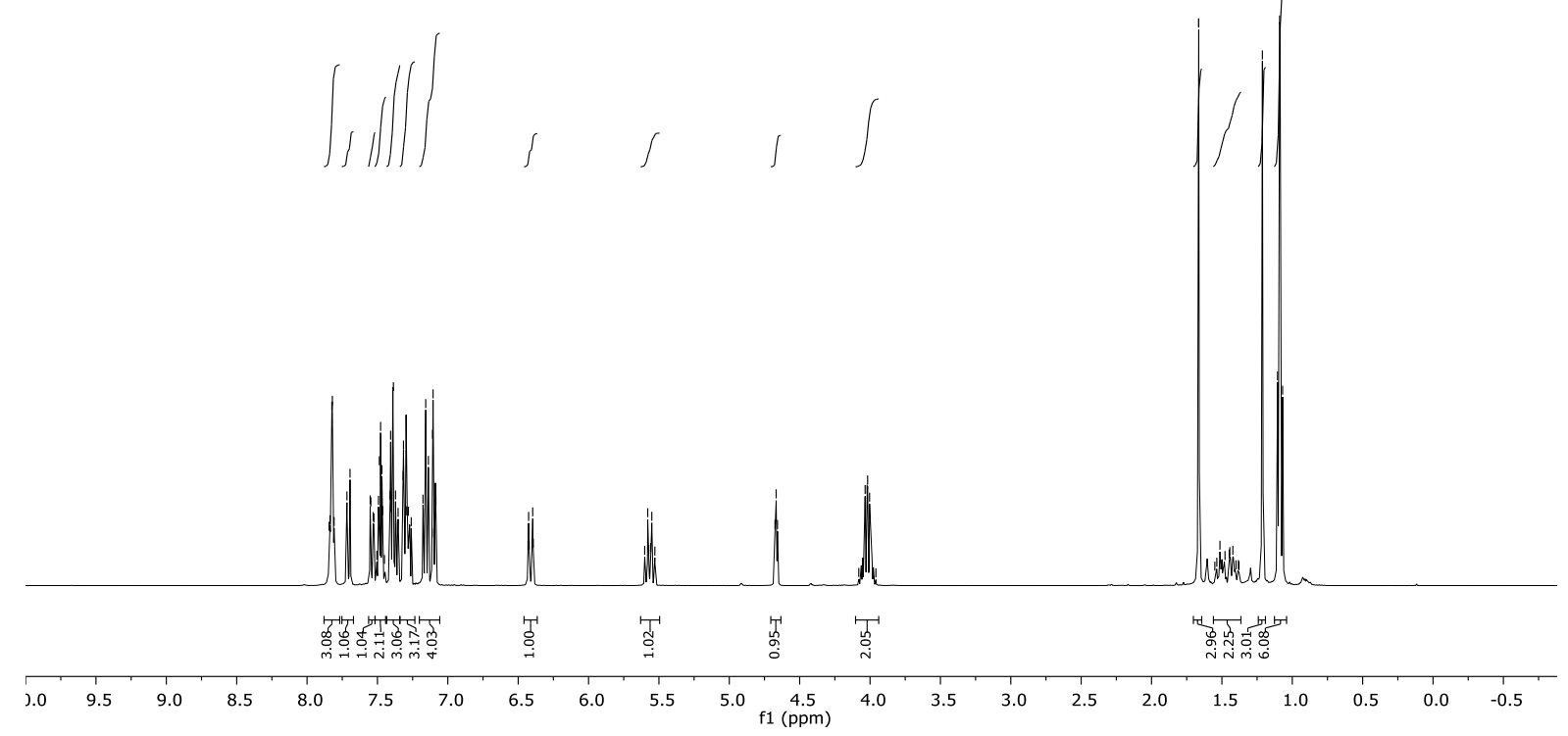

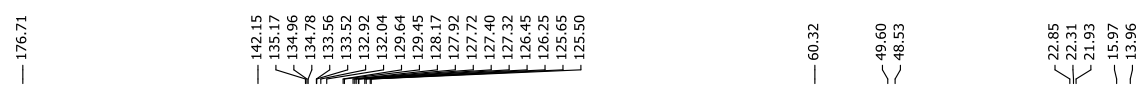

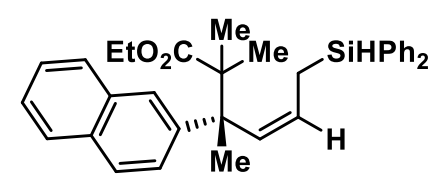

$(Z)-5$

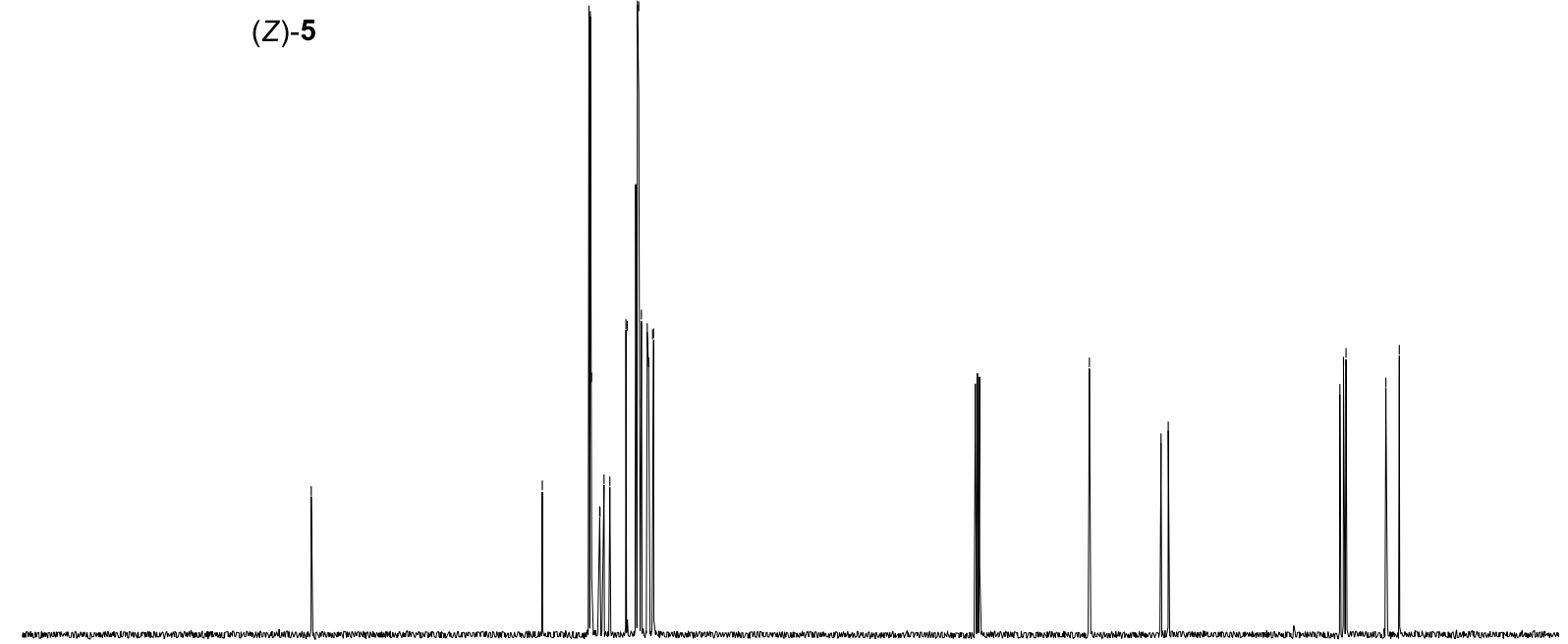

$\begin{array}{llllllllllllllllllllllllllllllll}210 & 200 & 190 & 180 & 170 & 160 & 150 & 140 & 130 & 120 & 110 & 100 & 90 & 80 & 70 & 60 & 50 & 40 & 30 & 20 & 10 & 0\end{array}$ 
<smiles>CCOC(=O)C(C)(C)[C@](C)(/C=C(\CN1CCCC1)c1ccccc1)c1ccc2ccccc2c1</smiles>

(Z)-6
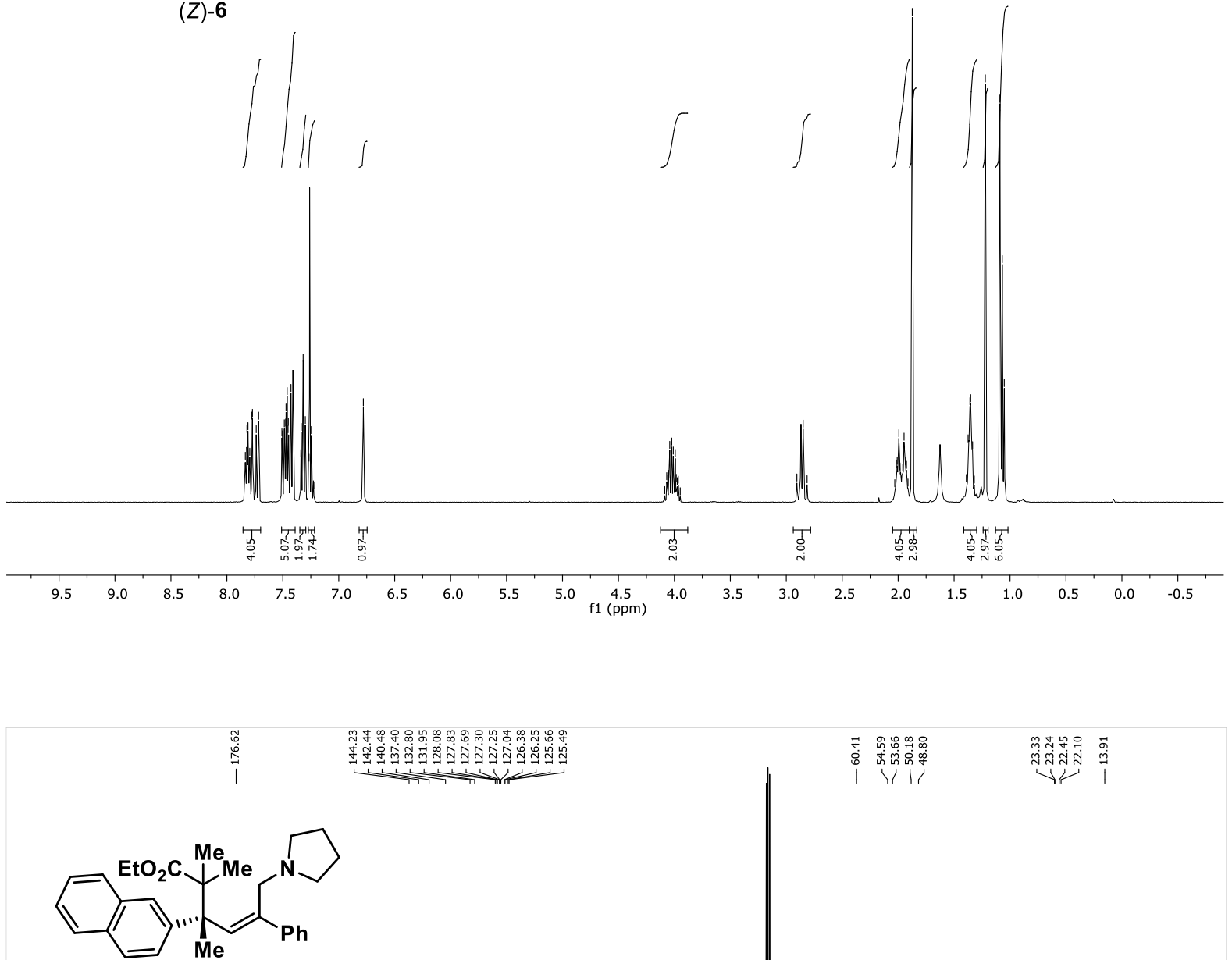

(Z)-6

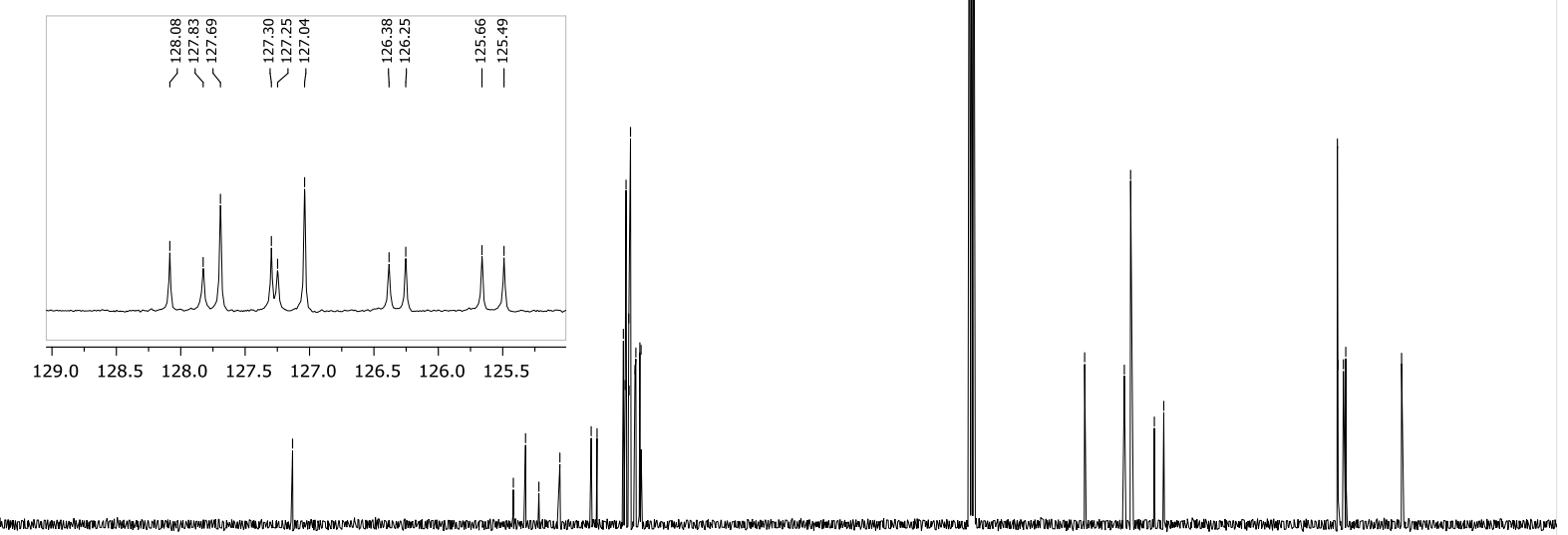

$\begin{array}{lllllllllll}210 & 200 & 190 & 180 & 170 & 160 & 150 & 140 & 130 & 120 & \begin{array}{l}110 \\ \mathrm{f} 1(\mathrm{ppm})\end{array}\end{array}$ 


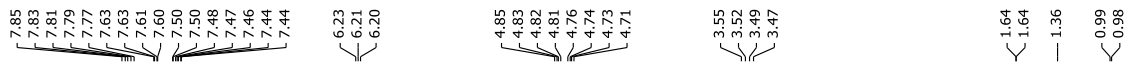

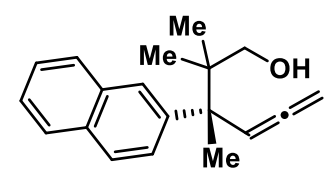

53

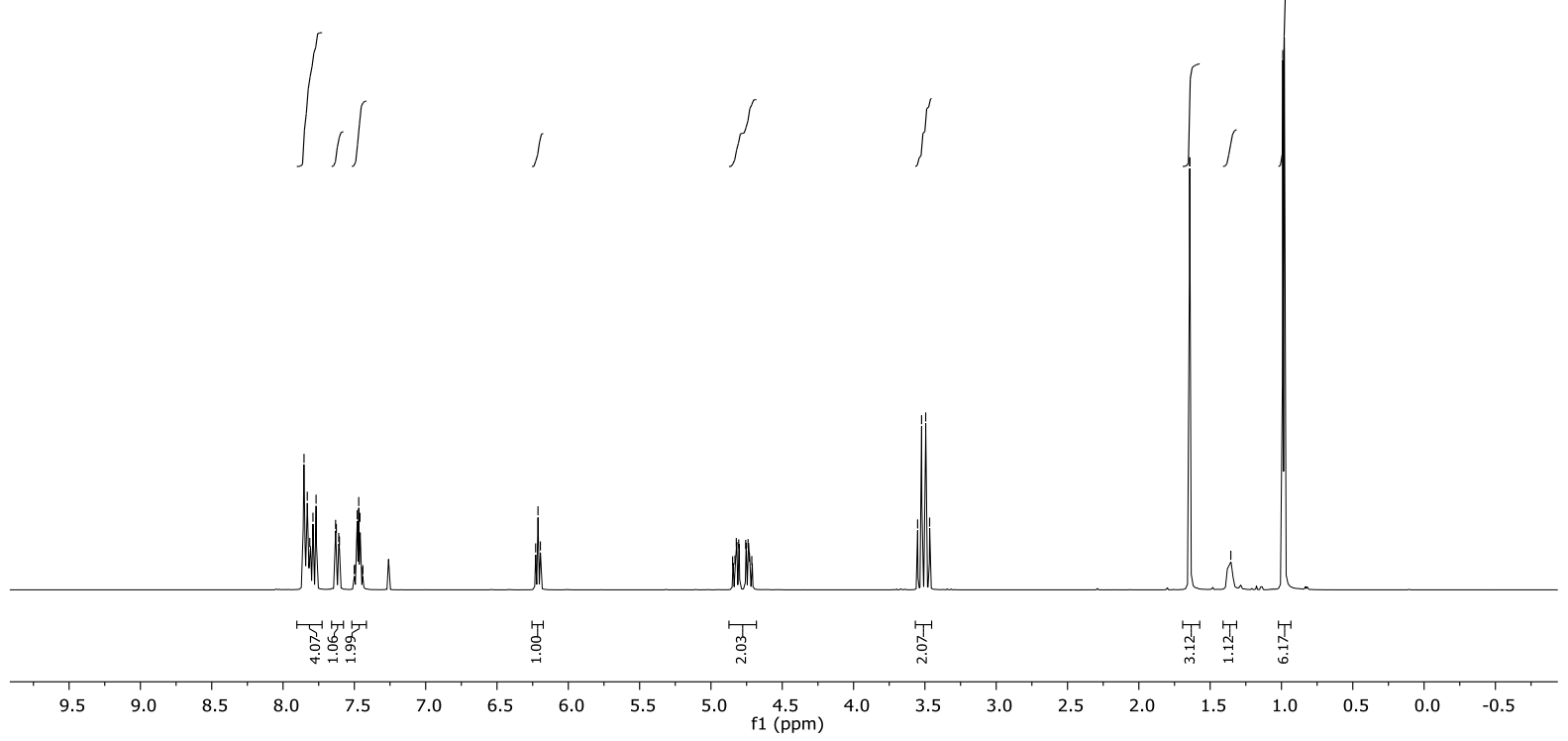

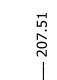

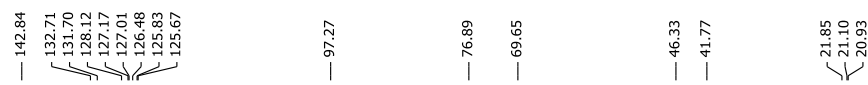<smiles>C=CC[C@](C)(c1ccc2ccccc2c1)C(C)(C)CO</smiles>

S3

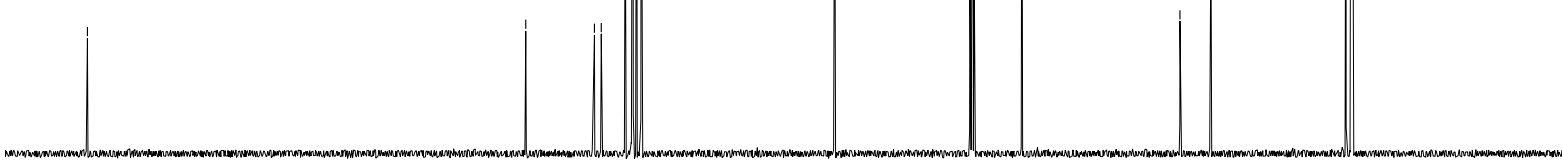

$\begin{array}{llllllllllllllllllllllllllllll} & 210 & 200 & 190 & 180 & 170 & 160 & 150 & 140 & 130 & 120 & 110 & 100 & 90 & 80 & 70 & 60 & 50 & 40 & 30 & 20 & 10 & 0 & -1\end{array}$ 
年

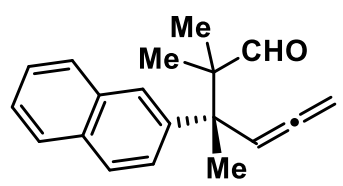

S4

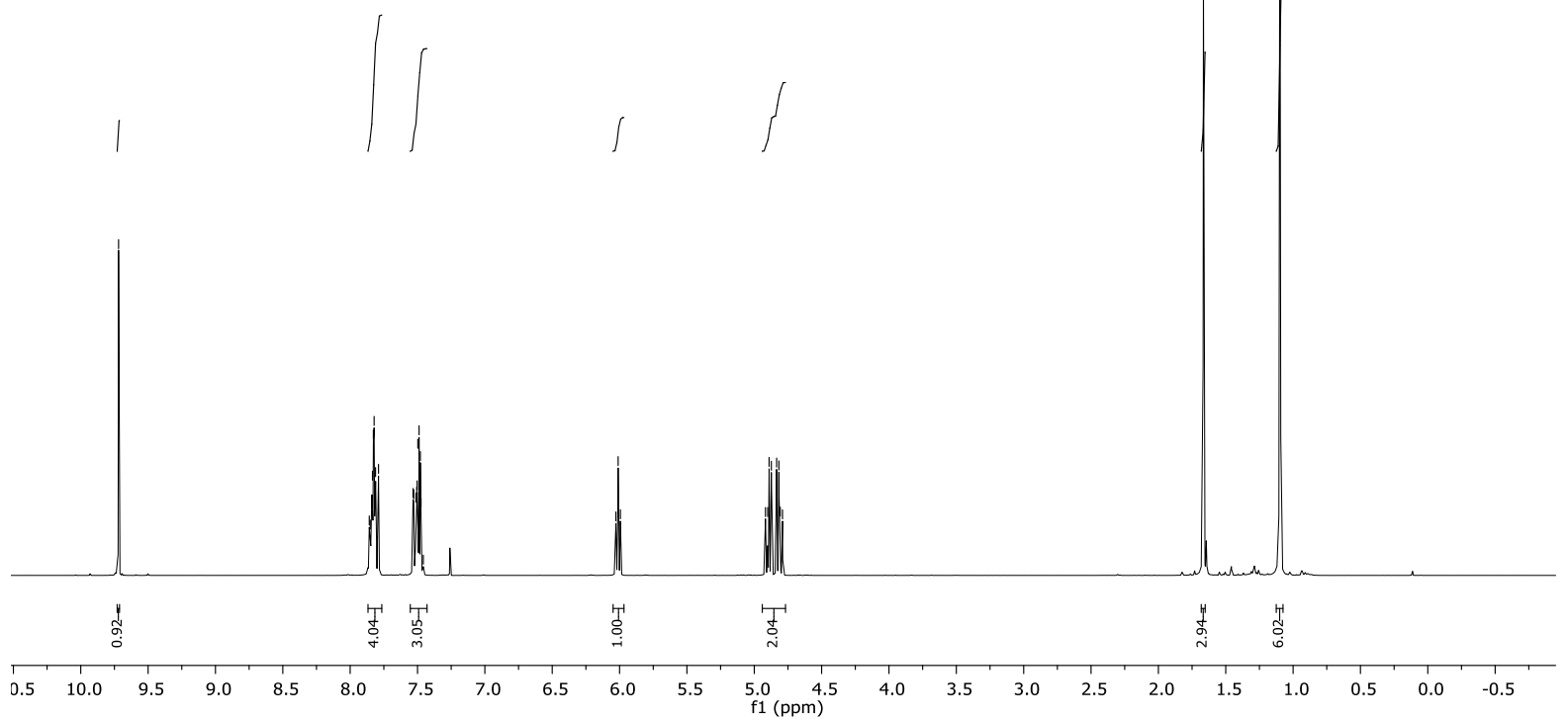

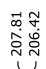

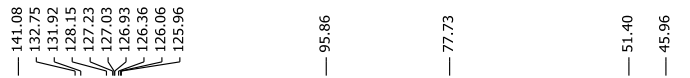

نั่

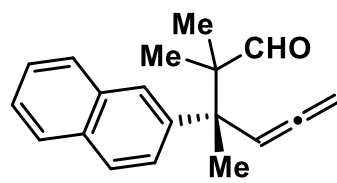

S4

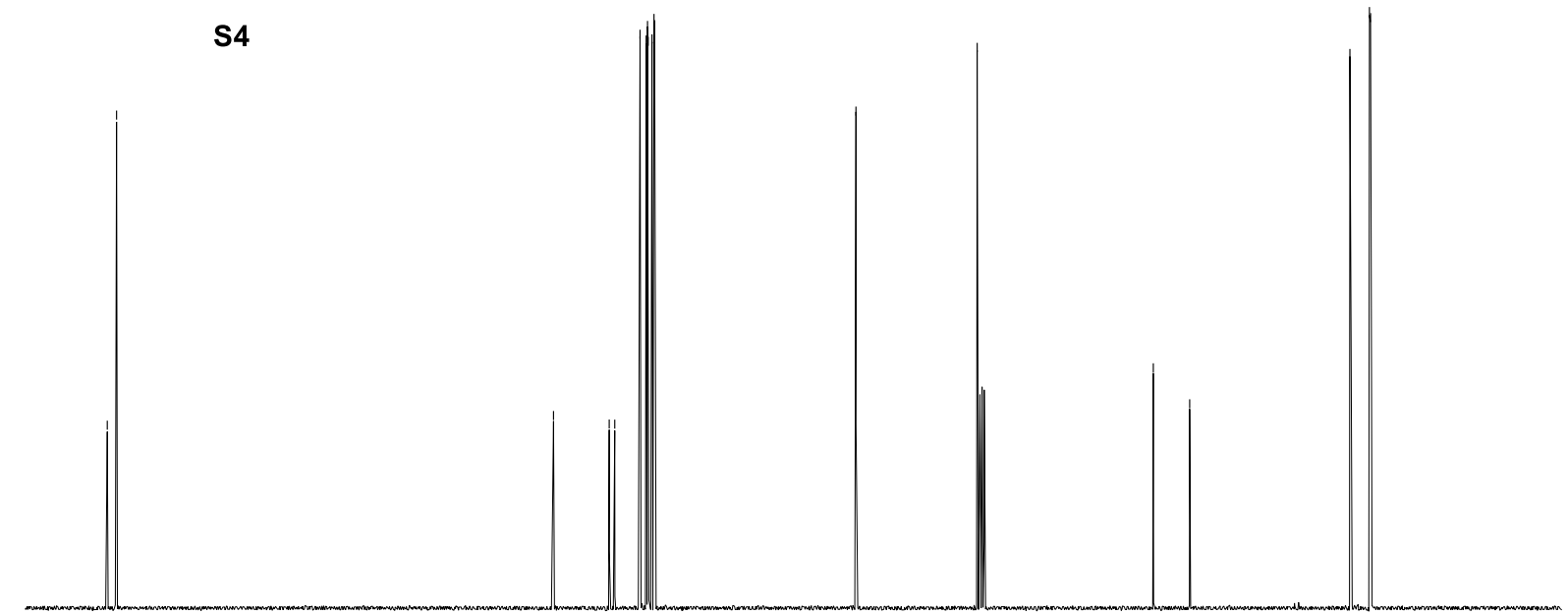

$\begin{array}{llllllllllllllllllllllllll}1 & 210 & 200 & 190 & 180 & 170 & 160 & 150 & 140 & 130 & 120 & 110 & 100 & 90 & 80 & 70 & 60 & 50 & 40 & 30 & 20 & 10 & 0\end{array}$ 


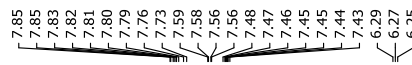

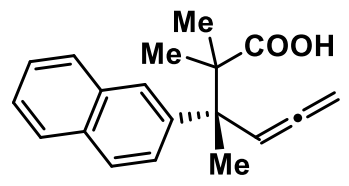

7
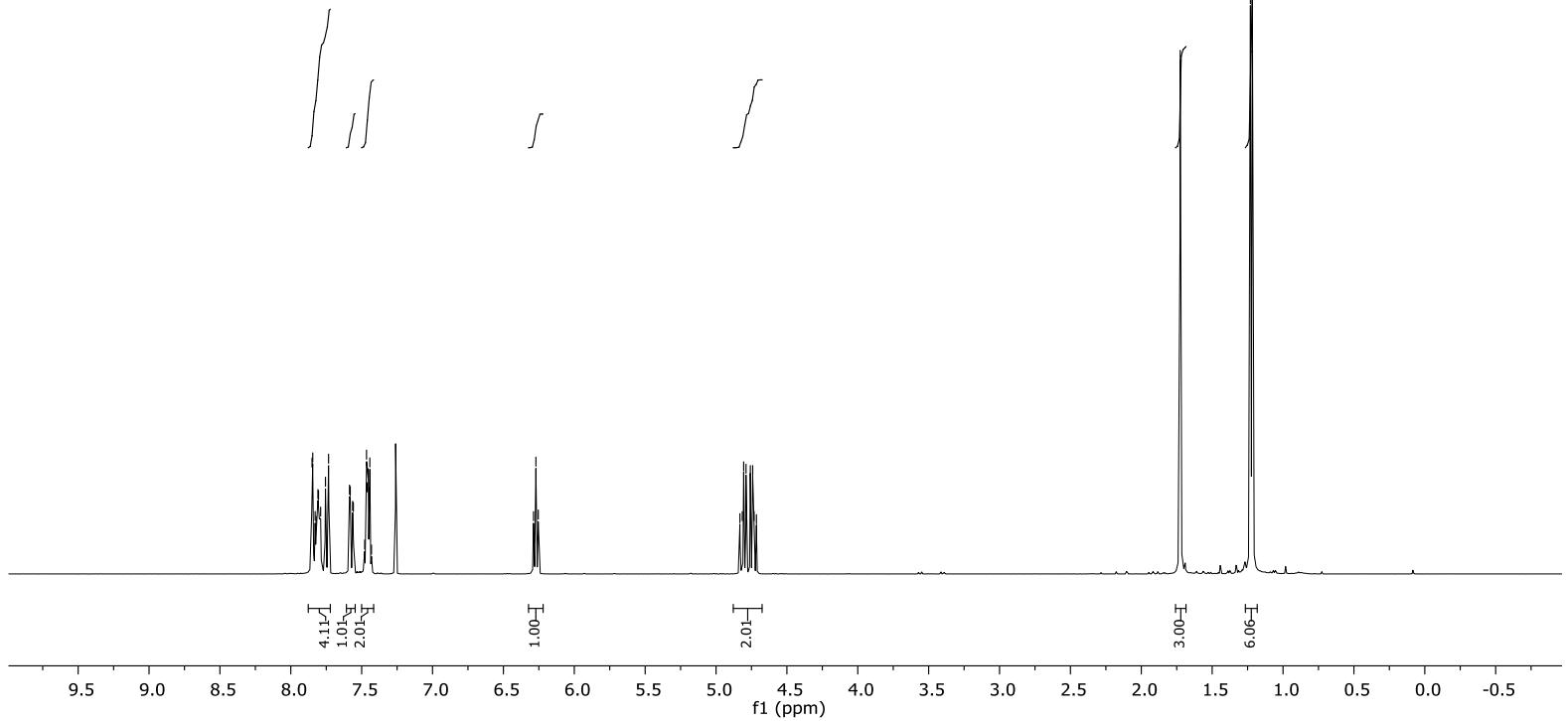

i

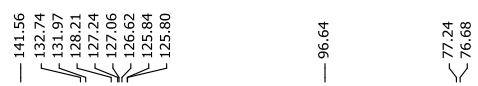

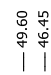

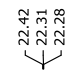

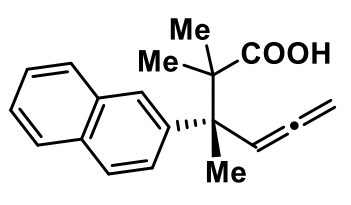

7

$\begin{array}{llllllllllll}220 & 210 & 200 & 190 & 180 & 170 & 160 & 150 & 140 & 130 & 120 & \begin{array}{l}110 \\ \mathrm{f} 1(\mathrm{ppm})\end{array}\end{array}$ 

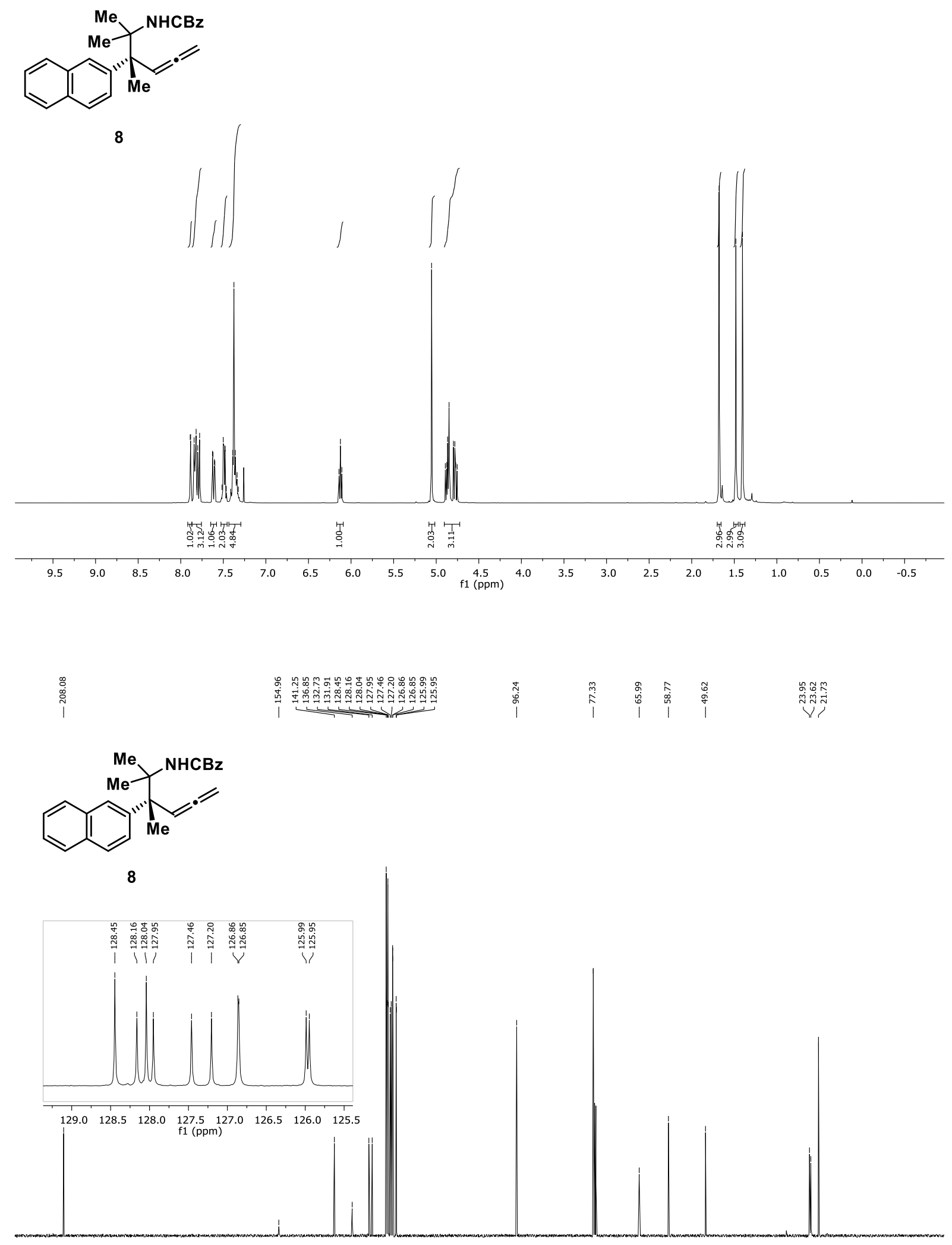

\begin{tabular}{llllllllllllllllllllllllllllll}
\hline 20 & 210 & 200 & 190 & 180 & 170 & 160 & 150 & 140 & 130 & 120 & 110 & 100 & 90 & 80 & 70 & 60 & 50 & 40 & 30 & 20 & 10 & 0 &
\end{tabular} 

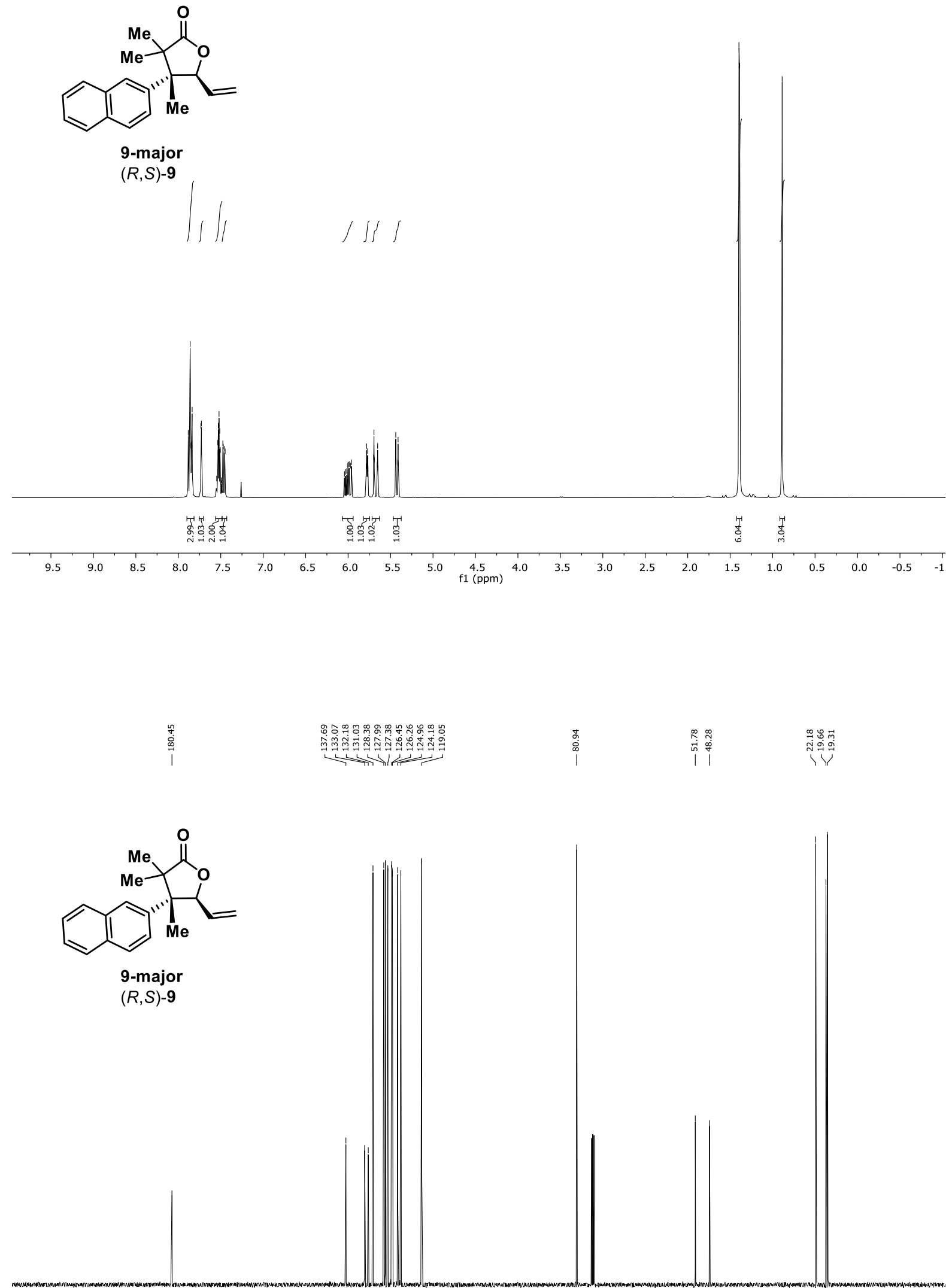

$\begin{array}{lllllllllll}210 & 200 & 190 & 180 & 170 & 160 & 150 & 140 & 130 & 120 & \begin{array}{l}110 \\ \mathrm{f} 1(\mathrm{ppm})\end{array}\end{array}$ 


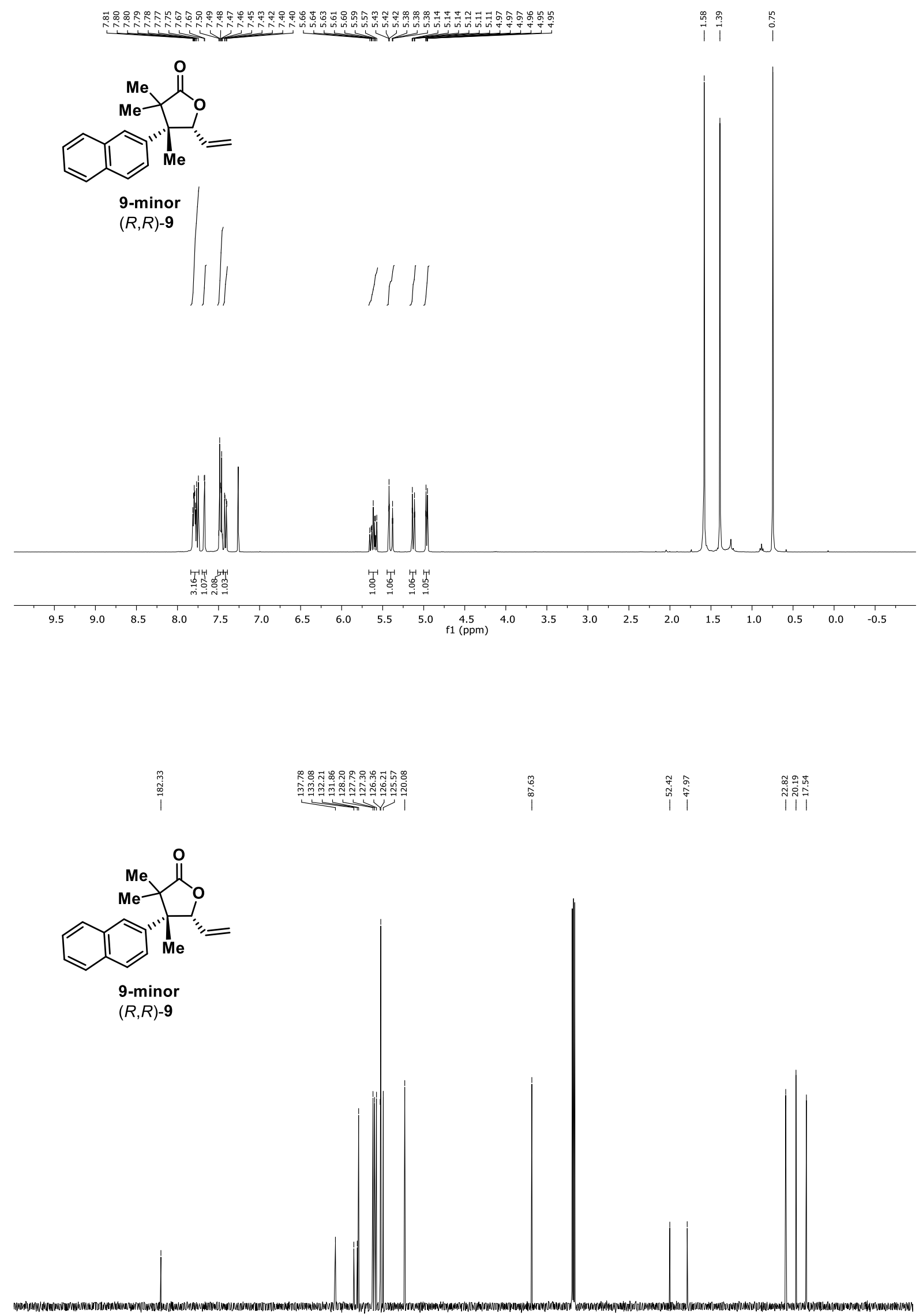

$\begin{array}{lllllllllll}210 & 200 & 190 & 180 & 170 & 160 & 150 & 140 & 130 & 120 & \begin{array}{c}110 \\ \mathrm{f} 1(\mathrm{ppm})\end{array}\end{array}$ 


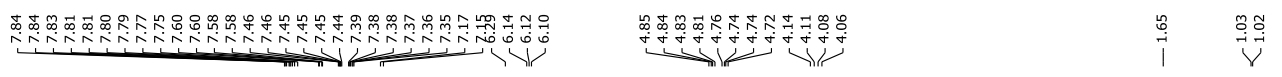<smiles>C=CC(C)(C)C(C)(C)COC(=O)Nc1ccc(Br)cc1</smiles>
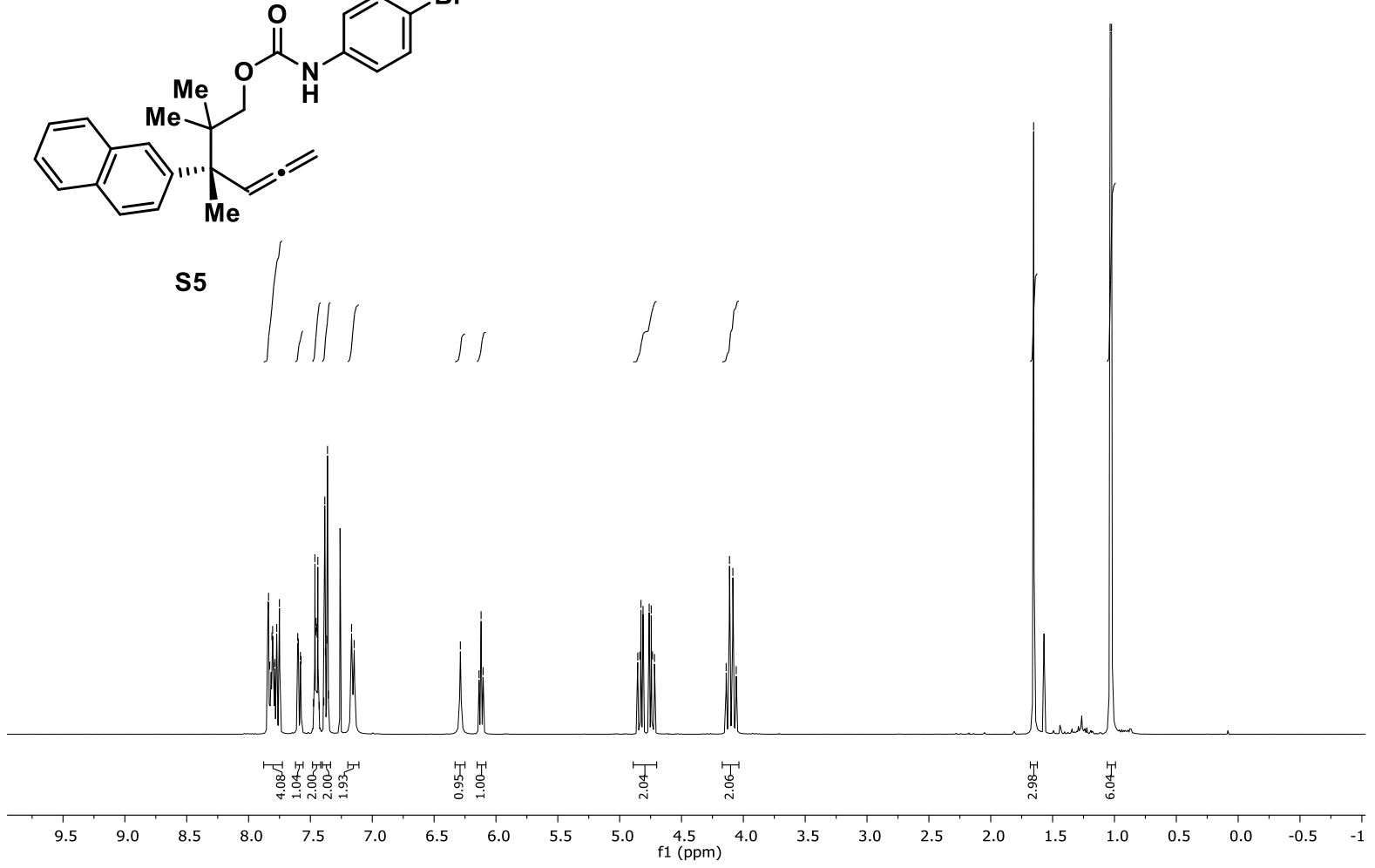

尊

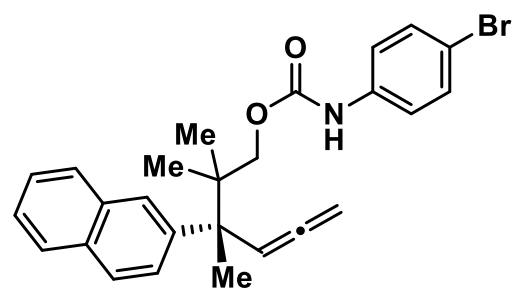

S5

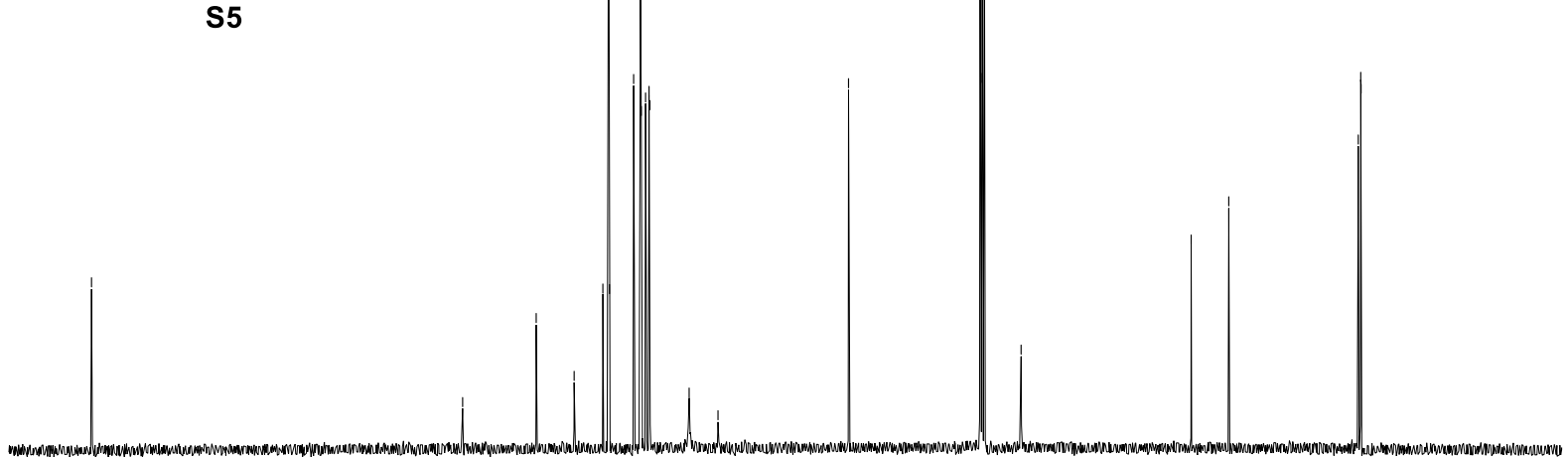

$\begin{array}{llllllllllllllllllllllllll}210 & 200 & 190 & 180 & 170 & 160 & 150 & 140 & 130 & 120 & \underset{\mathrm{f} 1(\mathrm{ppm})}{110} & 100 & 90 & 80 & 70 & 60 & 50 & 40 & 30 & 20 & 10 & 0\end{array}$ 


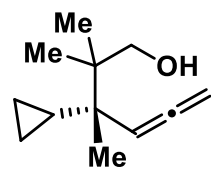

s6
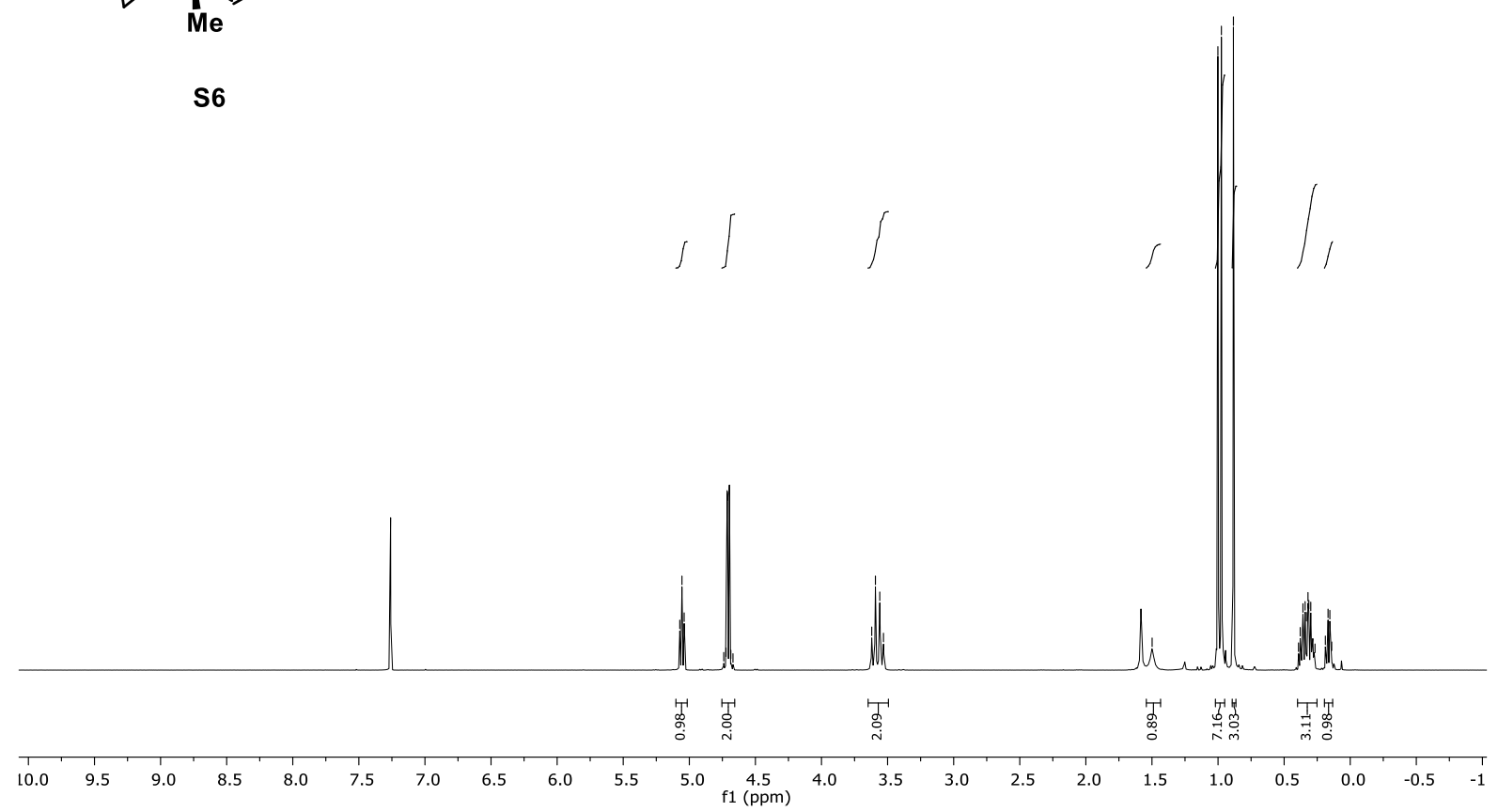

点

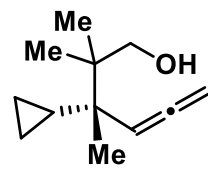

s6

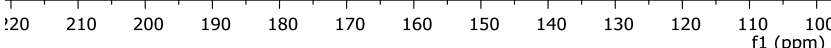




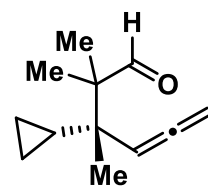

s7
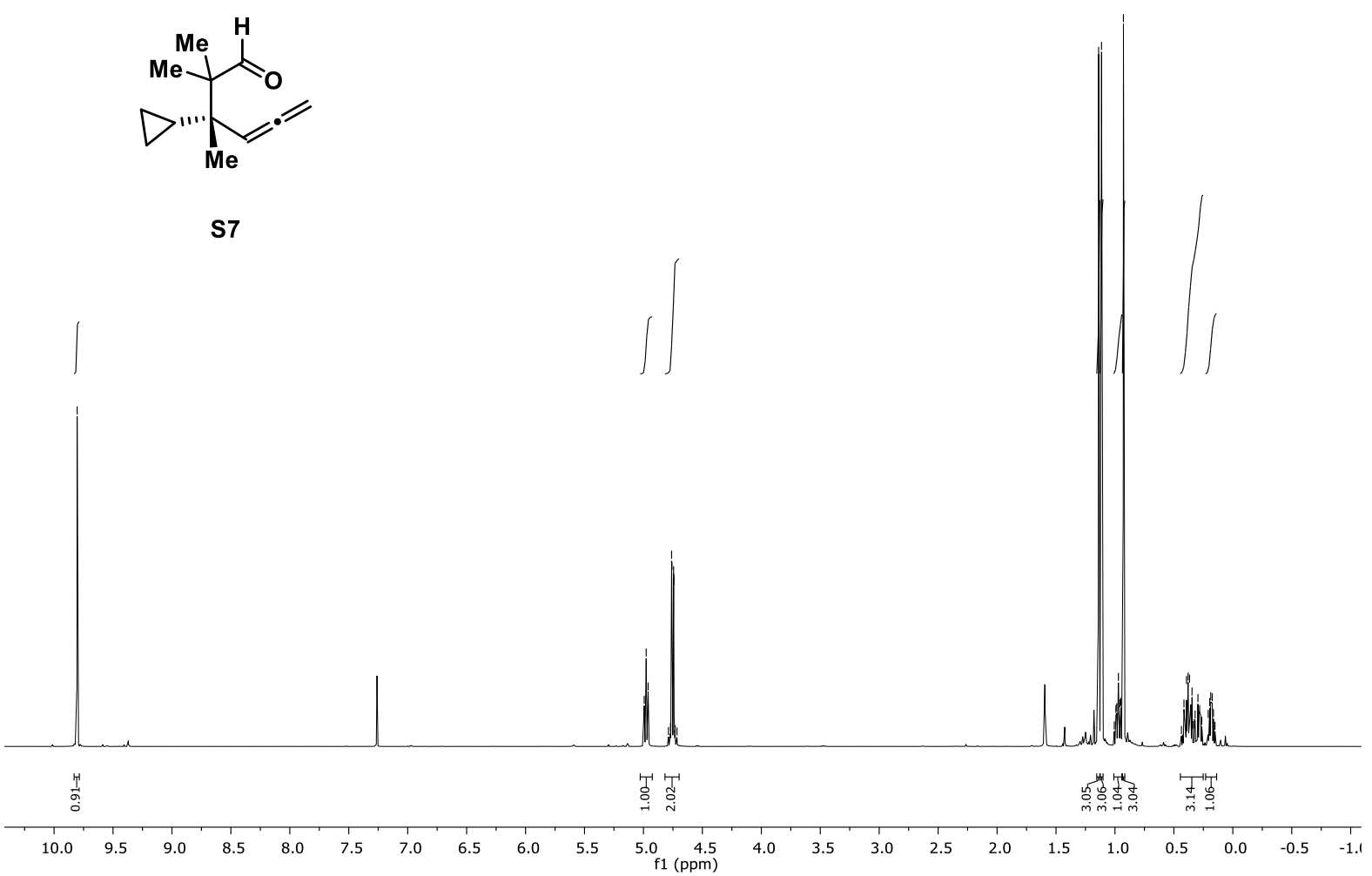

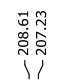

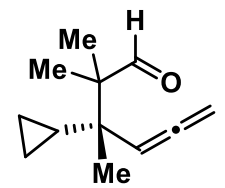

s7

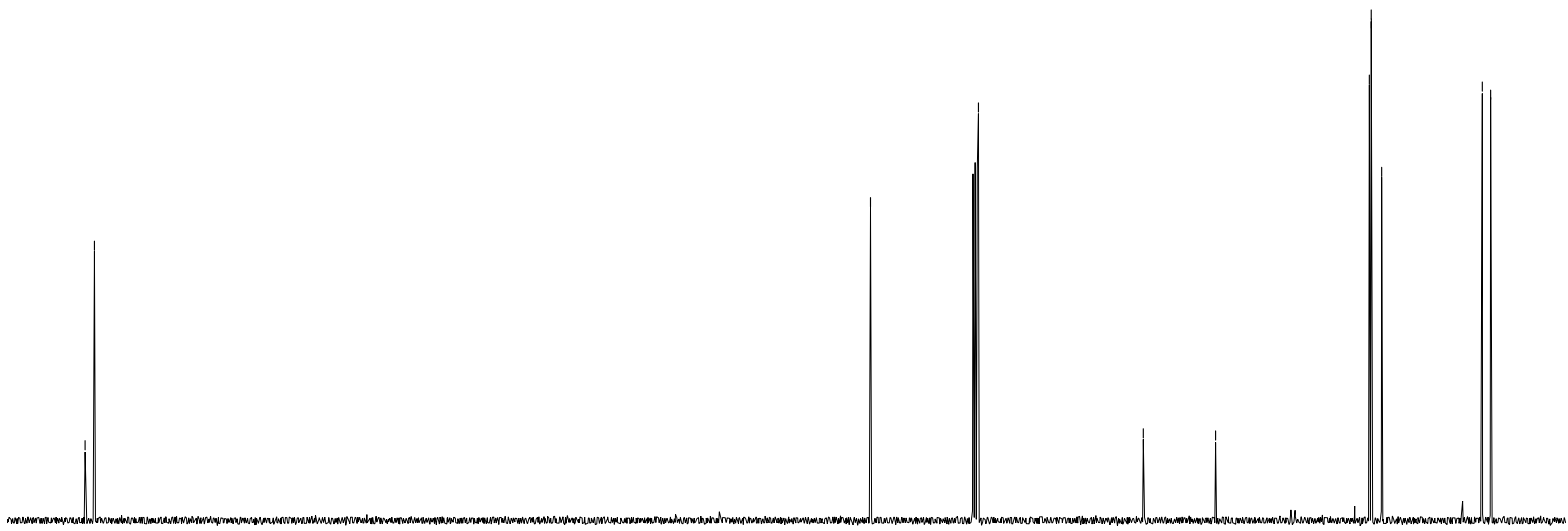

$\begin{array}{llllllllllllllllllllllll} & 210 & 200 & 190 & 180 & 170 & 160 & 150 & 140 & 130 & 120 & 110 & 100 & 90 & 80 & 70 & 60 & 50 & 40 & 30 & 20 & 10 & 0 & -1\end{array}$ 


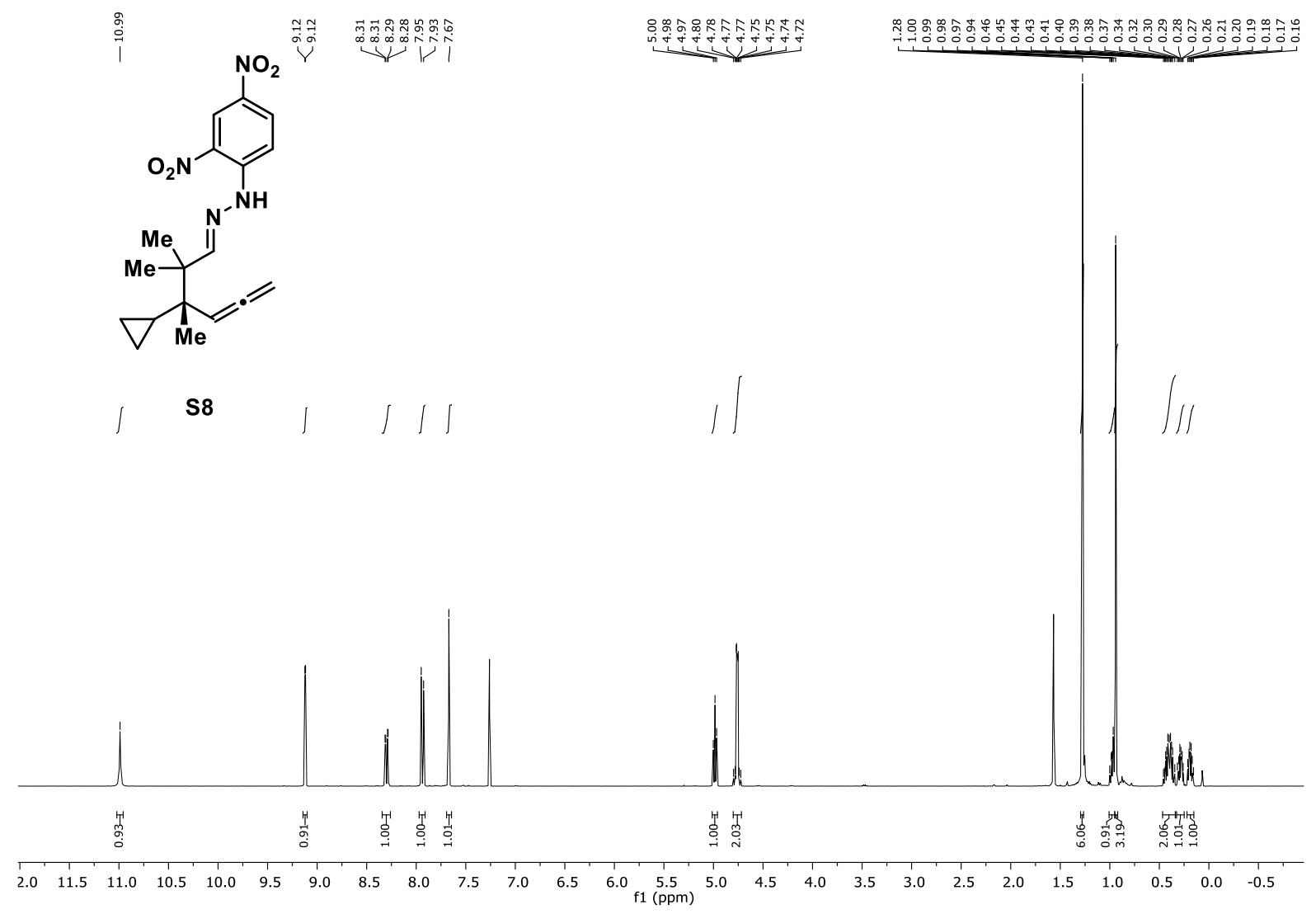

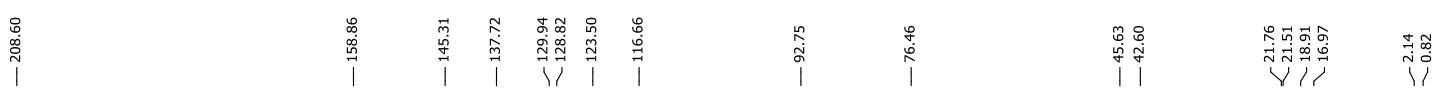<smiles>C=CC(C)(C)C(C)(C)C=NNc1ccc([N+](=O)[O-])cc1[N+](=O)[O-]</smiles>

58

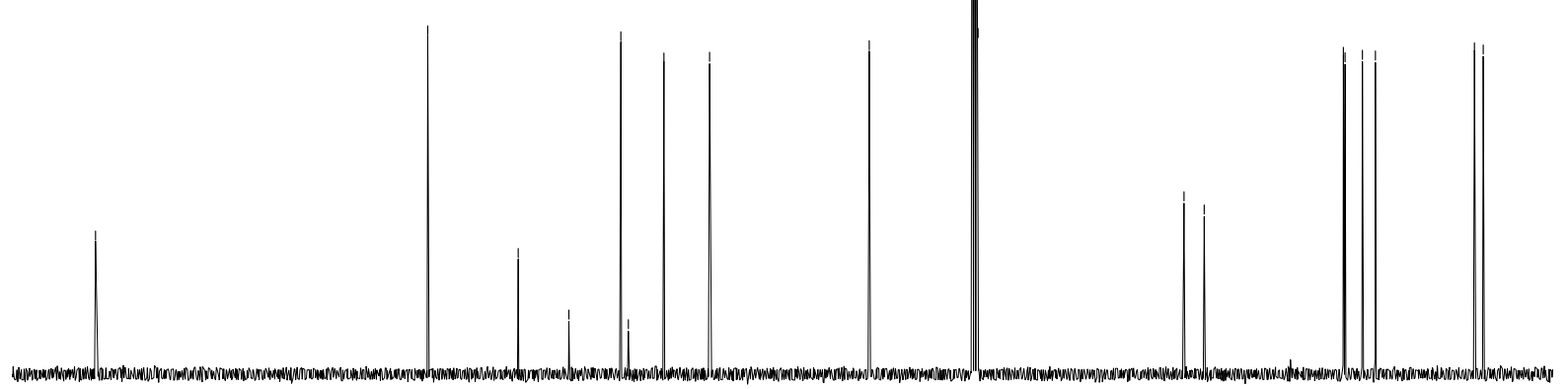

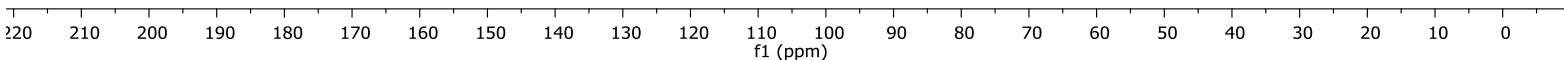



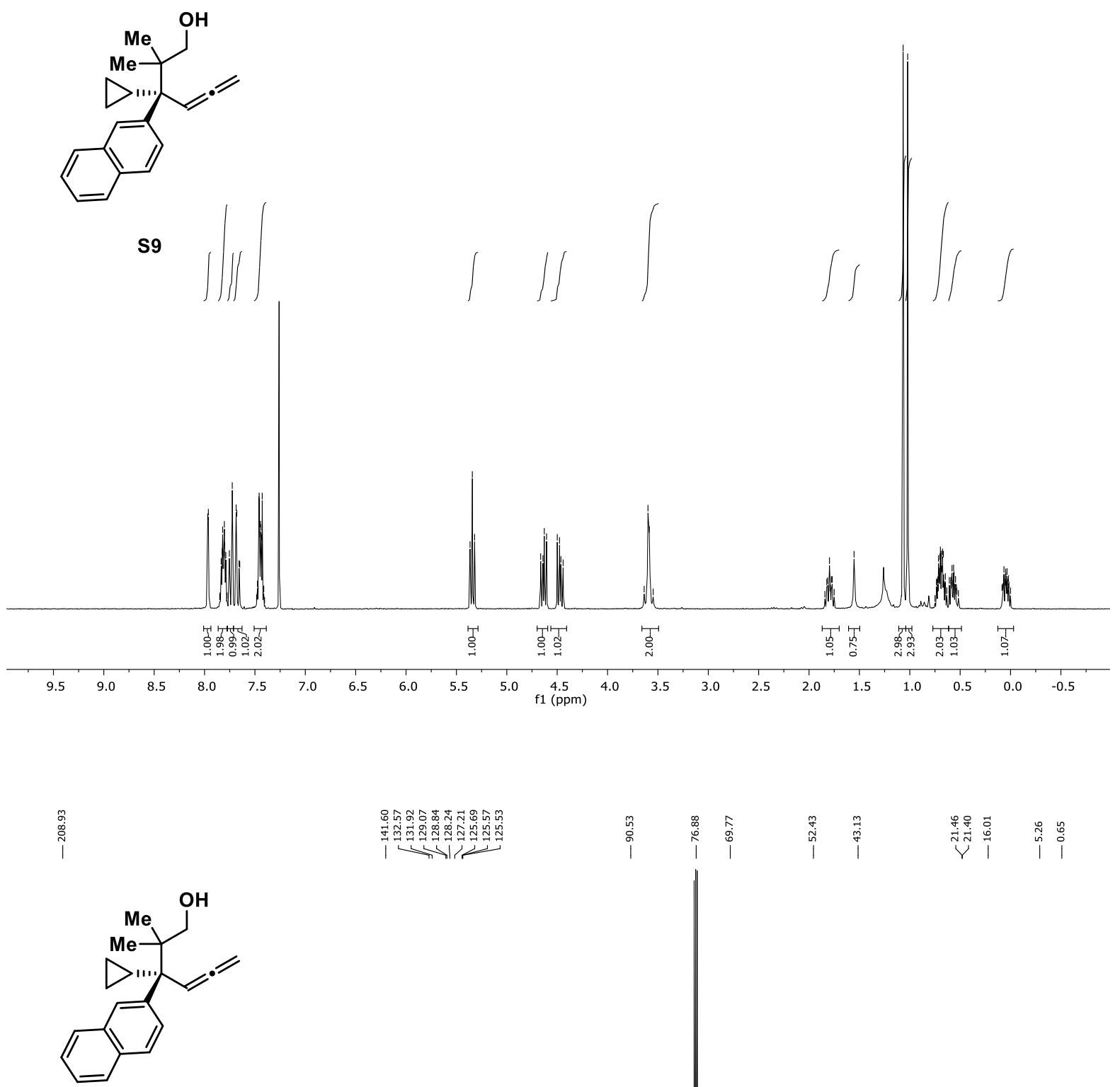

S9

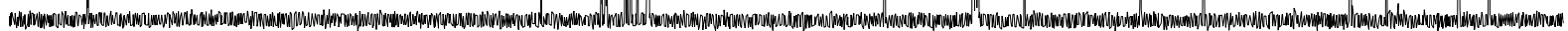

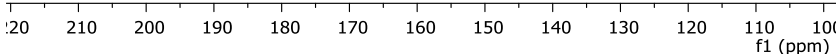



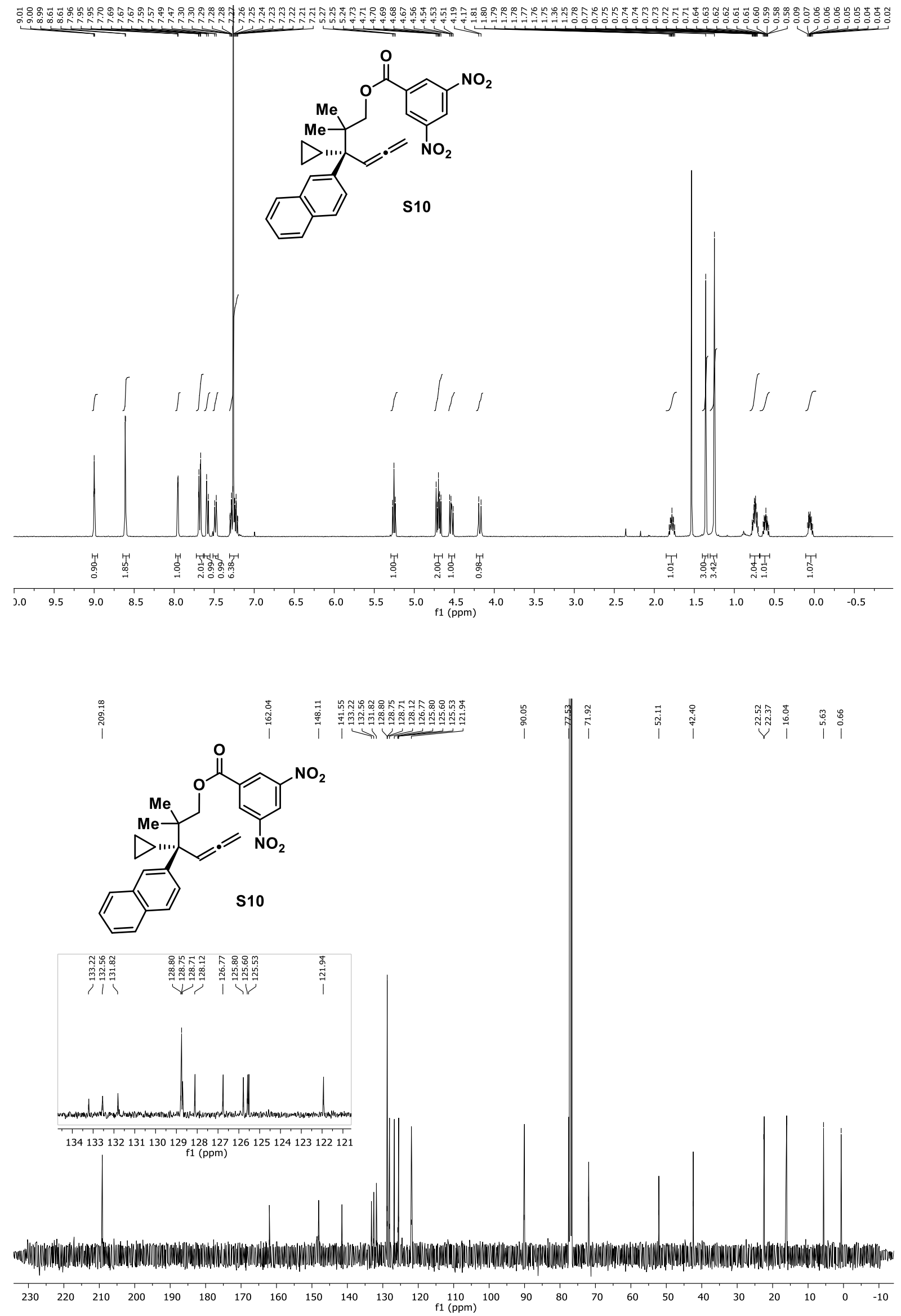


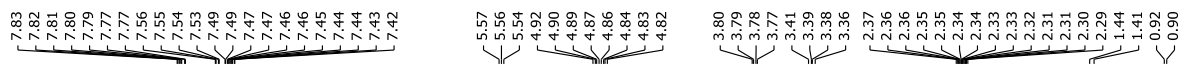

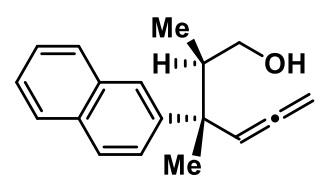

S11
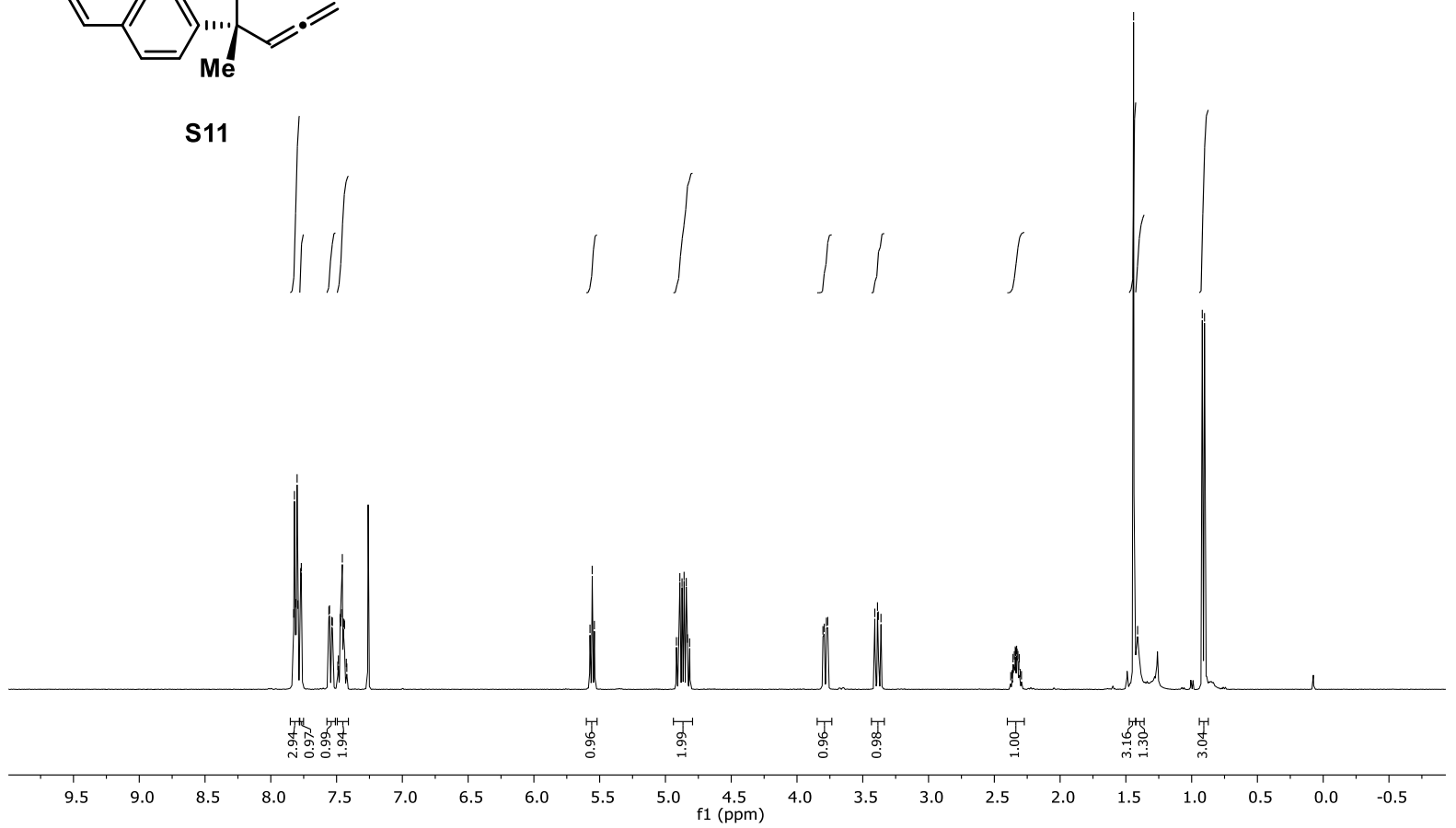

$\stackrel{\substack{0 \\ \stackrel{2}{i}}}{\stackrel{2}{i}}$

م.

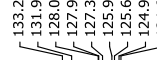

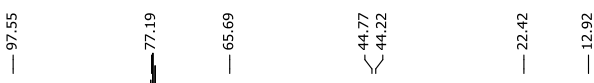

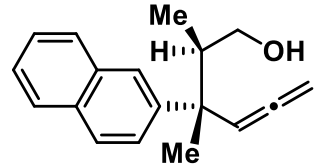

S11

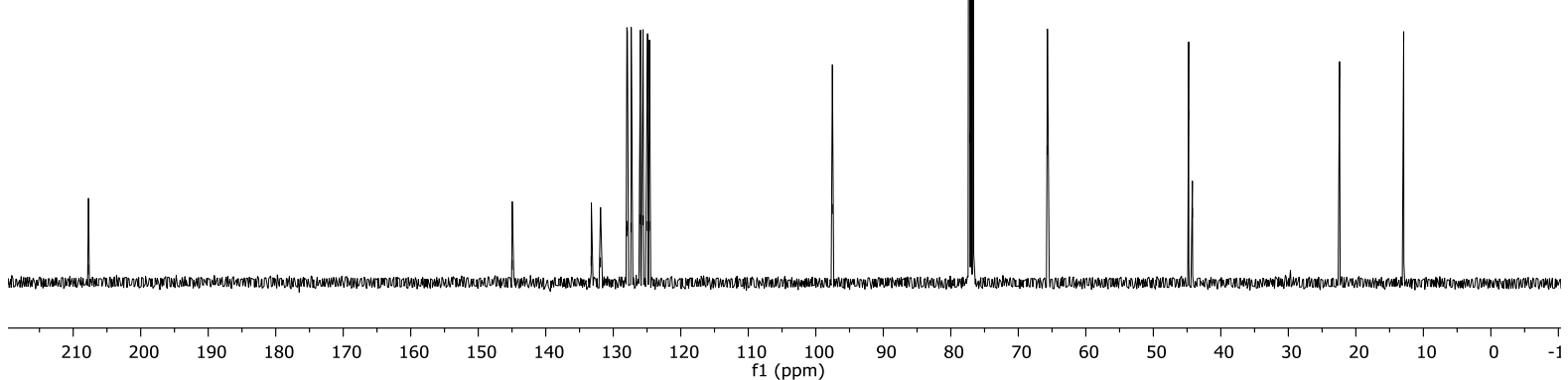




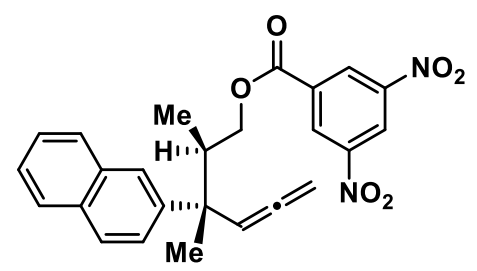

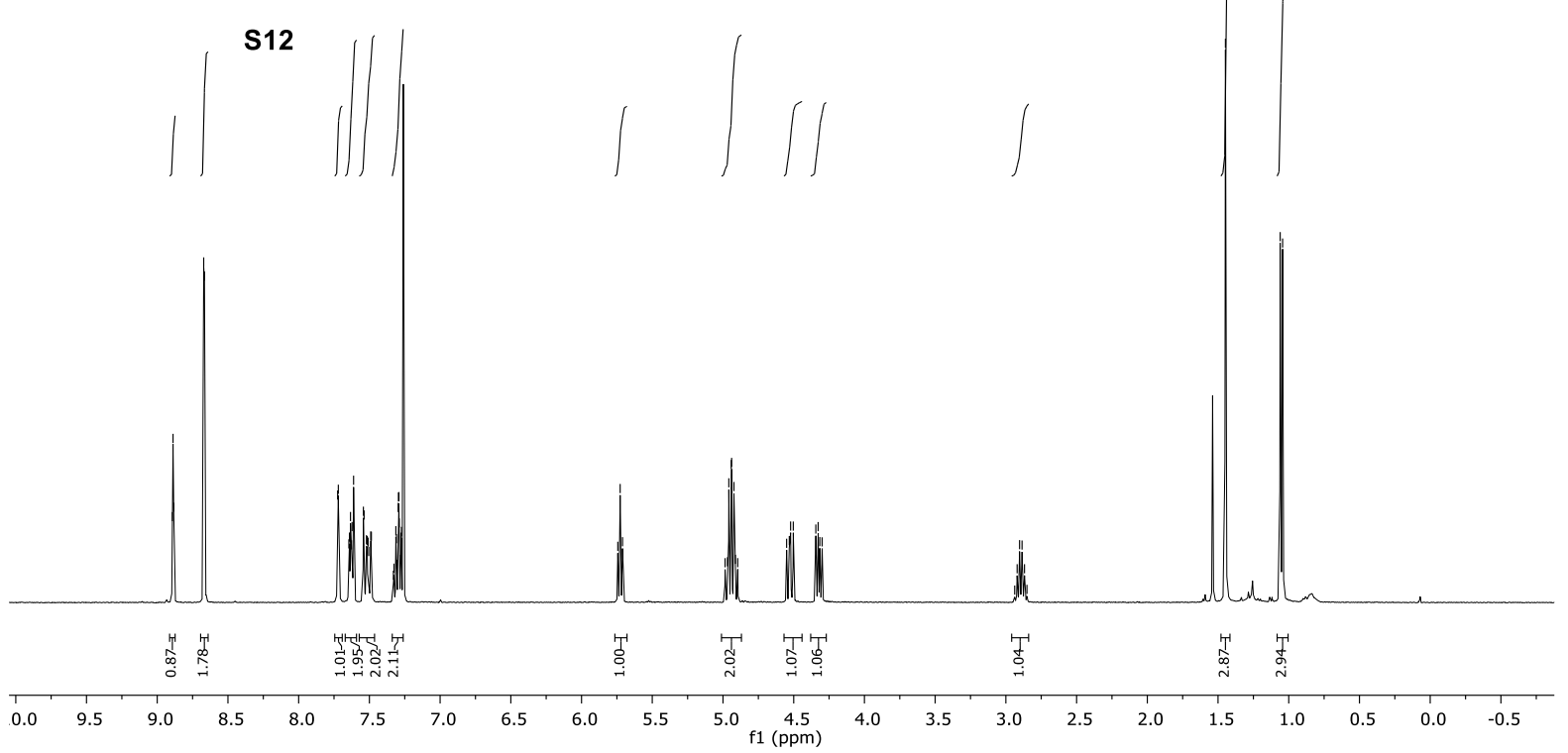

$$
\begin{gathered}
\overrightarrow{0} \\
\substack{0 \\
\stackrel{1}{1}} \\
1
\end{gathered}
$$<smiles>C=CC(C)[C@@](C)(c1ccc2ccccc2c1)[C@@H](C)COC(=O)c1cc([N+](=O)[O-])cc([N+](=O)[O-])c1</smiles>

S12

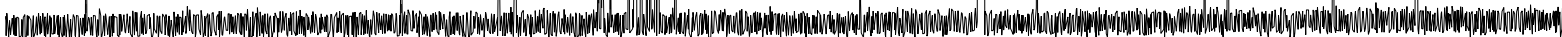

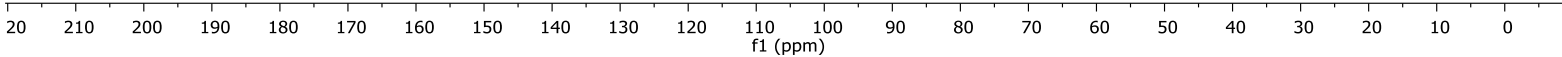




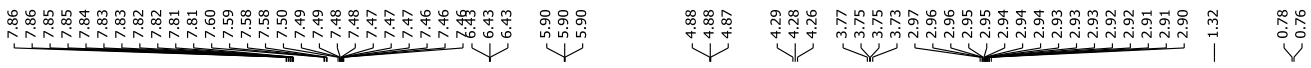

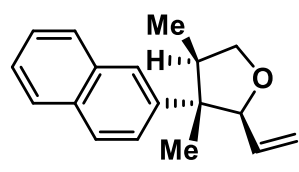

$(R, S)-\mathbf{S} 13$
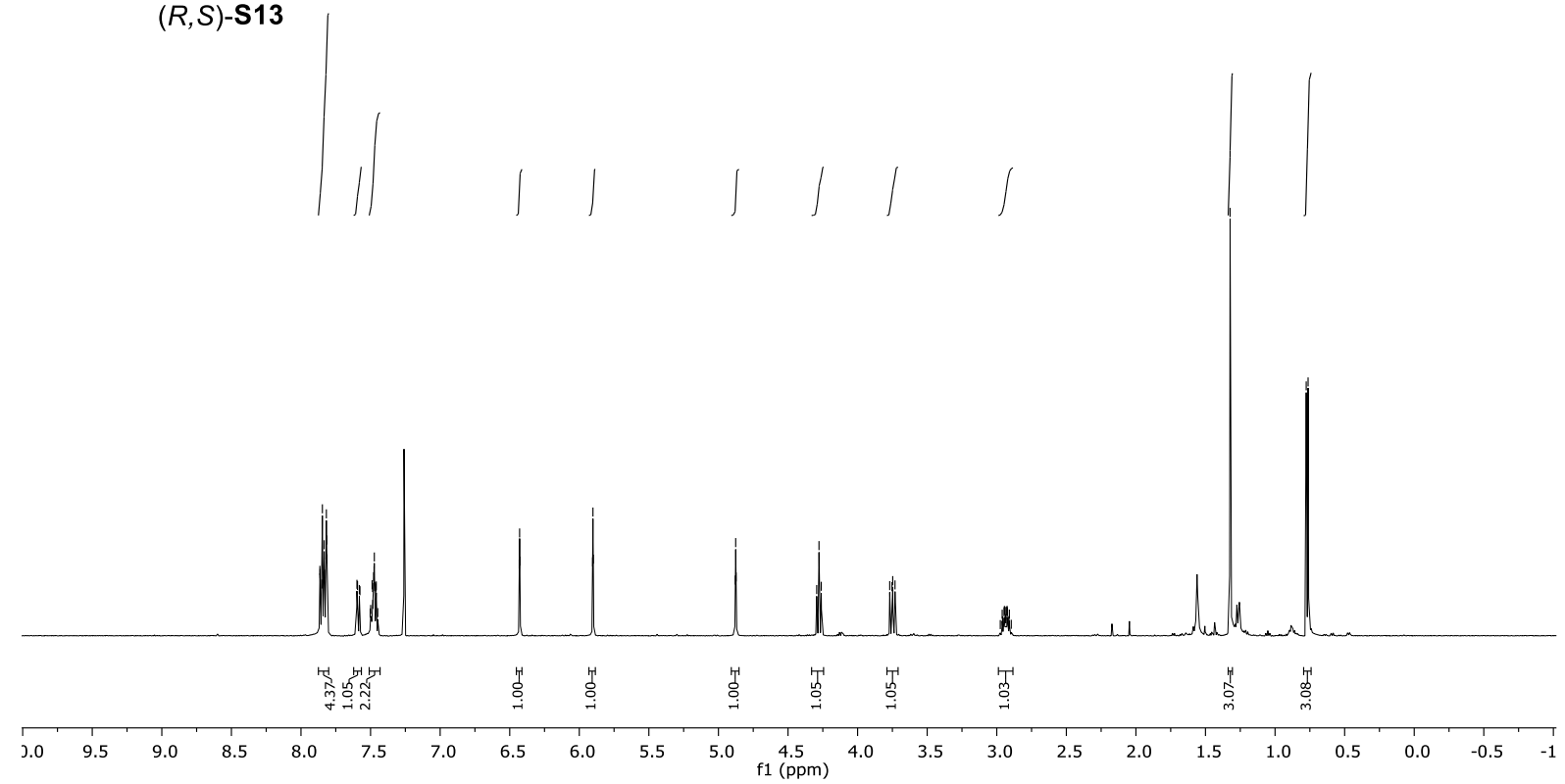

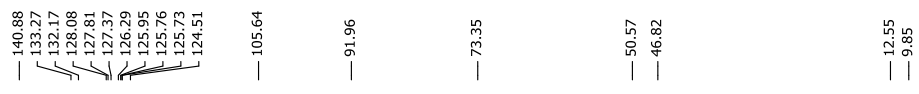

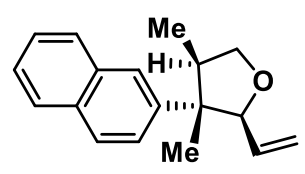

$(R, S)-\mathrm{S} 13$

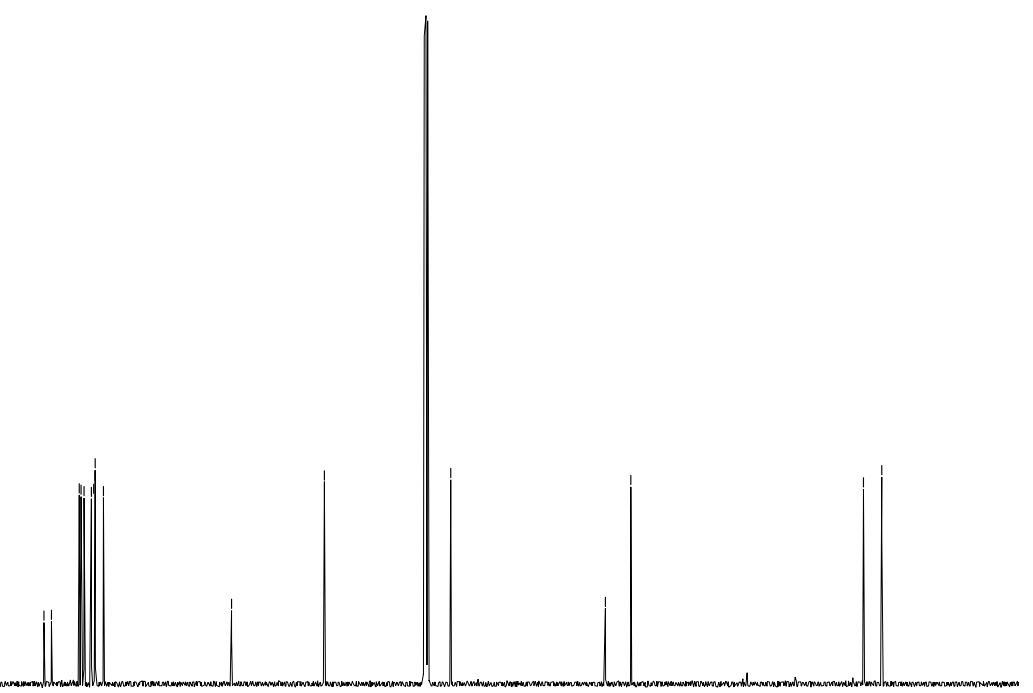

\begin{tabular}{llllllllllllllllllllllllll}
\hline 20 & 210 & 200 & 190 & 180 & 170 & 160 & 150 & 140 & 130 & 120 & 110 & 100 & 90 & 80 & 70 & 60 & 50 & 40 & 30 & 20 & 10 & 0 & -1
\end{tabular} 


\section{References}

${ }^{1}$ Still, W.C.; Kahn, M.; Mitra, A. J. Org. Chem. 1978, 43, 2923-2925.

${ }^{2}$ Sheldrick, G. M. Acta Cryst. 2008, A64, 112-122.

${ }^{3}$ Sheldrick, G. M. Acta Cryst. 2015, A71, 3-8.

${ }^{4}$ a) Palatinus, L.; Chapuis, G. J. J. Appl. Cryst. 2007, 40, 786-790; b) Palatinus,L.; Prathapa, S. J.; van Smaalen, S. J. Appl. Cryst. 2012, 45, 575-580.

${ }^{5}$ Dolomanov,O. V.; Bourhis, L. J.; Gildea, R. J.; Howard, J. A. K.; Puschmann, H. J. Appl. Cryst. 2009, 42, 339-341.

${ }^{6}$ Farrugia, L. J. J. Appl. Cryst. 2012, 45, 849-854.

${ }^{7}$ Isomura, M.; Petrone, D. A.; Carreira, E. M. J. Am. Chem. Soc. 2019, 141, 4738-4748.

${ }^{8}$ Luo, H.; Ma, S. Eur. J. Org. Chem. 2013, 15, 3041-3048.

${ }^{9}$ McAdam, C. A.; McLaughlin, M. G.; Johnston, A. J. S.; Chen, J.; Walter, M. W.; Cook, M. J. Org. Biomol. Chem. 2013, 11, 4488-4502.

${ }^{10}$ Yamashita, Y.; Cui, Y.; Xie, P.; Kobayashi, S. Org. Lett. 2015, 17, 6042-6045.

${ }^{11}$ Mothe, S. R.; Kothandaraman, P.; Rao, W.; Chan, P. W. H. J. Org. Chem. 2011, 76, 25212531.

${ }^{12}$ Inamoto, Y.; Kaga, Y.; Nishimoto, Y.; Yasuda, M.; Baba, A. Chem. Eur. J. 2014, 20, 1166411668.

${ }^{13}$ Ojima, I.; Kumagai, M.; Nagai, Y. J. Organomet. Chem. 1976, 111, 43-60.

${ }^{14}$ Gatzenmeier, T.; Kaib, P. S. J.; Lingnau, J. B.; Goddard, R.; List, B. Angew. Chem. Int. Ed. 2018, 57, 2464-2468.

${ }^{15}$ Ireland, R. E.; Wipf, P.; Armstrong III, J. D. J. Org. Chem. 1991, 56, 650-657.

${ }^{16}$ Wenzel, A. G.; Jacobsen, E. N. J. Am. Chem. Soc. 2002, 124, 12964-12965.

${ }^{17}$ Asako, S.; Ishikawa, S.; Takai, K. ACS Catal. 2016, 6, 3387-3395.

${ }^{18}$ Shimizu, I.; Tsuji, J. Chem. Lett. 1984, 13, 233-236.

${ }^{19}$ Lumbroso, A.; Abermil, N.; Breit, B. Chem. Sci. 2012, 3, 789-793. 